\title{
Development of Cross Correlation for Objective Comparison of Profiles
}

\author{
FINAL REPORT \\ Report 2002-36 \\ Steven M. Karamihas \\ University of Michigan Transportation Research Institute
}

SPONSORED BY

FHWA Western Federal Lands Division

July 2002 

Technical Report Documentation Page

\begin{tabular}{|c|c|}
\hline $\begin{array}{l}\text { 1. Report No. } \\
\text { UMTRI-2002-36 }\end{array}$ & 3. Recipient's Catalog No. \\
\hline \multirow{3}{*}{$\begin{array}{l}\text { 4. Title and Subtitle } \\
\text { Development of Cross Correlation for Objective Comparison } \\
\text { of Profiles }\end{array}$} & 5. Report Date July 2002 \\
\hline & $\begin{array}{r}\text { 6. Performing Organization Code } \\
42954\end{array}$ \\
\hline & 8. Performing Organization Report No. \\
\hline $\begin{array}{l}\text { 7. Author(s) } \\
\text { S. M. Karamihas and T. D. Gillespie }\end{array}$ & UMTRI-2002-36 \\
\hline 9. Performing Organization Name and Address & 10. Work Unit No. (TRAIS) \\
\hline $\begin{array}{l}\text { The University of Michigan Transportation Research Institute } \\
\text { 2901 Baxter Road }\end{array}$ & 11. Contract or Grant No. \\
\hline Ann Arbor, Michigan 48109 & 13. Type of Report and Period Covered \\
\hline $\begin{array}{l}\text { 12. Sponsoring Agency Name and Address } \\
\text { U.S. Department of Transportation } \\
\text { FHWA Western Land Highway Division }\end{array}$ & $\begin{array}{l}\text { Final Report } \\
\text { Nov } 2001-\text { Nov } 2002\end{array}$ \\
\hline $\begin{array}{l}610 \text { East 5th Street } \\
\text { Vancouver, WA 98661-3801 }\end{array}$ & 14. Sponsoring Agency Code \\
\hline
\end{tabular}

15. Supplementary Notes

16. Abstract

Standard methods of comparing two profile measurements usually place tolerances on agreement between individual elevation values over a broad waveband. A weakness of this approach is the emphasis on long wavelength content. In most road profiles, the amplitude of elevation is roughly proportional to wavelength. Thus, long-wavelength features in the road dominate the elevation profile, even though short wavelength features are often just as relevant to vehicle response. This often prevents the detection of the most important measurement problems.

This report presents a procedure for rating the agreement between profile measurements using the cross correlation function. The method is capable of rating agreement between profiles after the content is isolated to a given waveband of interest, such as that of the International Roughness Index. The conventional cross correlation method is customized to assess agreement between profile shape and overall roughness level. Thus, a high rating depends on proper measurement of the overall roughness and its spatial distribution.

The method is demonstrated for the purpose of rating agreement between profile measurements and deducing their longitudinal triggering offset. This method is intended for application in profiler comparison studies as well as profiler certification testing programs. Threshold correlation values are developed for qualification as research class, project class, and network class.

\begin{tabular}{|l|l|}
\hline 17. Key Words & 8. Distribution Statement
\end{tabular}

Road profile measurement, road roughness, No restrictions. This document is available to profiler verification, longitudinal profile the public through the National Technical Information Service, Springfield, Virginia 22161.

\begin{tabular}{|c|c|l|l|}
\hline $\begin{array}{c}\text { 19. Security Classif. (of this report) } \\
\text { Unclassified }\end{array}$ & $\begin{array}{c}\text { 20. Security Classif. (of this page) } \\
\text { Unclassified }\end{array}$ & $\begin{array}{l}\text { 21. No. of Pages } \\
146\end{array}$ & 22. Price \\
\hline
\end{tabular}





\section{TABLE OF CONTENTS}

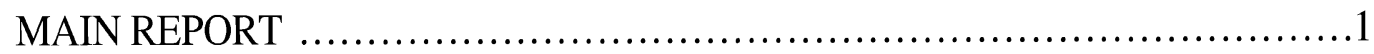

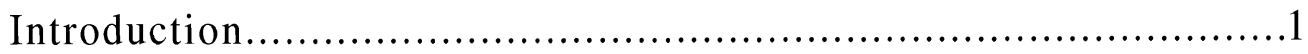

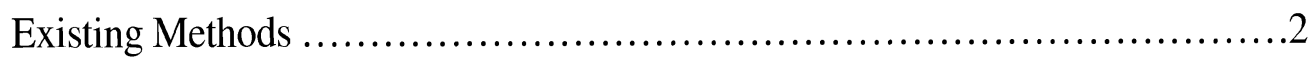

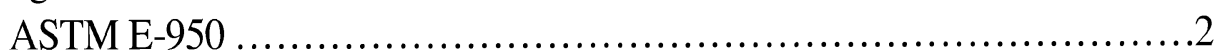

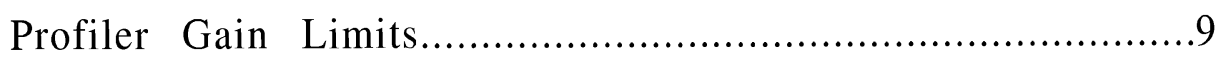

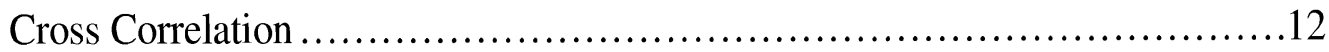

Theoretical Development ..............................................12

Synchronization .......................................................

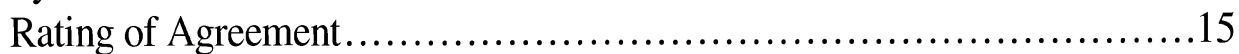

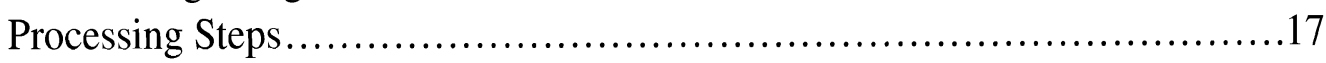

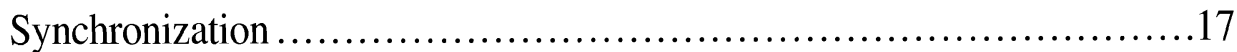

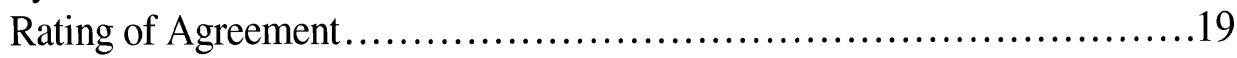

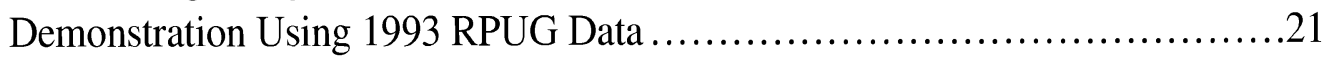

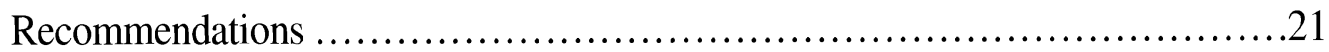

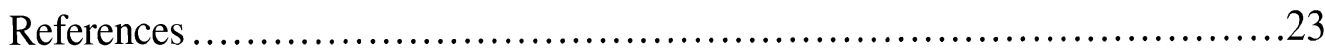

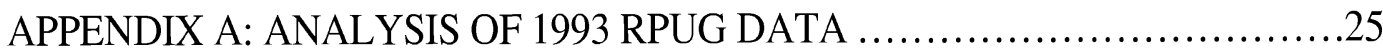

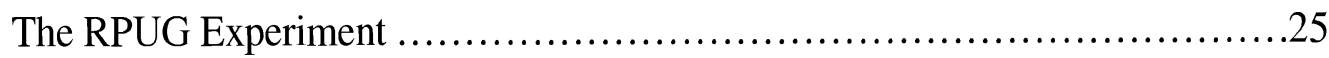

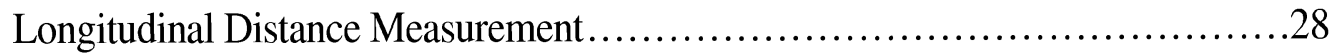

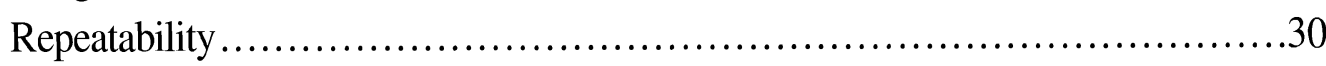

International Roughness Index ........................................ 30

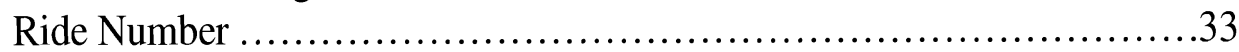

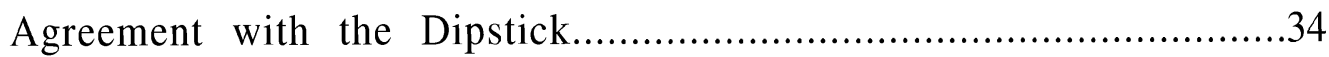

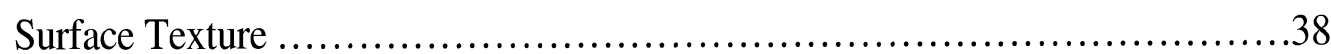

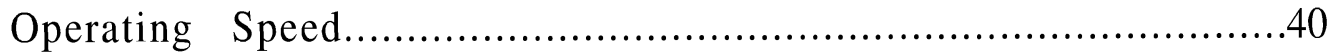

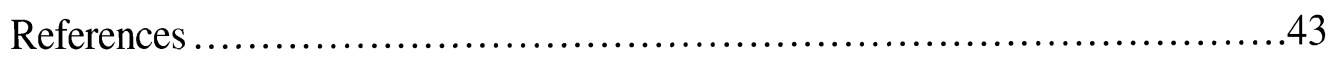

APPENDIX B: CROSS CORRELATION RESULTS, 1993 RPUG STUDY .......45

APPENDIX C: INTERPRETATION OF 1993 RPUG CROSS

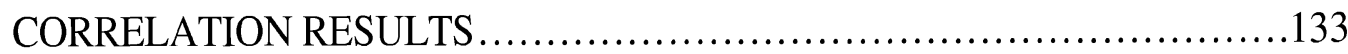

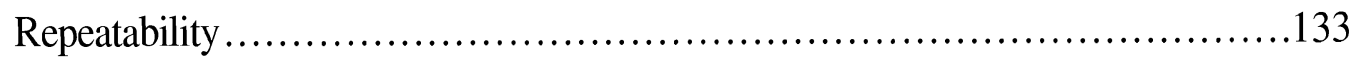

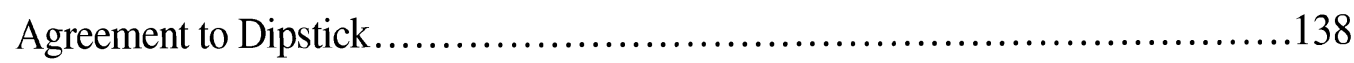

Agreement Between any Pair of Measurements.................................140 


\section{LIST OF FIGURES}

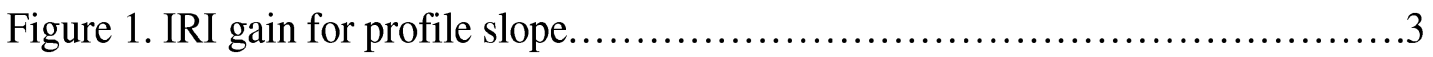

Figure 2. IRI gain for profile elevation...............................................

Figure 3. Theoretical error in IRI versus wavelength at Class I and II...................5

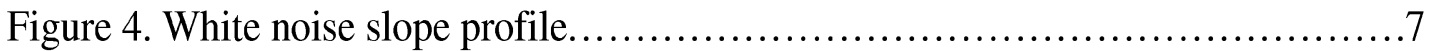

Figure 5. Profile smoothed with a 3 -meter moving average........................... 7

Figure 6. Absolute bias level of smoothed profiles.....................................

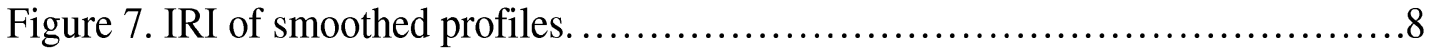

Figure 8. Profile comparison using transfer functions, after Prem. (8).................. 9

Figure 9. Coherence between road profiles.................................................11

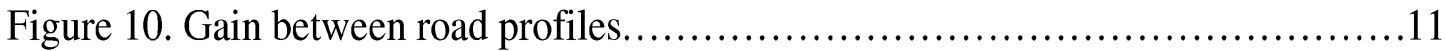

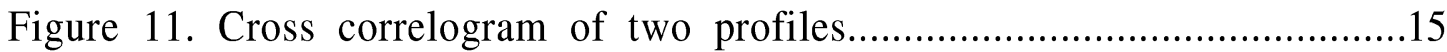

Figure 12. Cross correlogram of two profiles with periodic content...................15

Figure 12 . Three highly correlated repeat measurements. ............................17

Figure 13. Three moderately correlated repeat measurements..........................17

Figure 15. IRI agreement associated with cross correlation level.......................21

Figure A-1. Repeatability of the ProRut. ...............................................

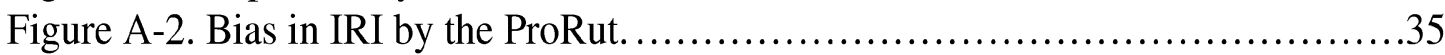

Figure A-3. Bias in agency-built ultrasonic profilers from the RPUG..................37

Figure A-4. Bias in commercial ultrasonic profilers from the RPUG....................37

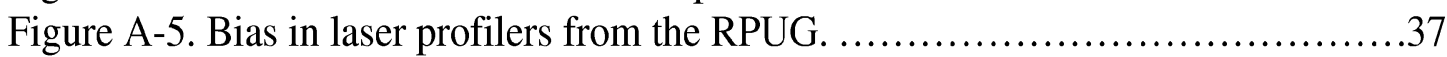

Figure A-6. Bias in optical profilers from the RPUG.................................38

Figure A-7. Bias in ultrasonic profilers from the RPUG in Pennsylvania.................39

Figure C-1. Comparison of IRI scatter and cross correlation............................136

Figure C-2. Poorly-correlated IRI filter output............................................137

Figure C-3. Well-correlated IRI filter output...........................................137

Figure C-4. Comparison of RMS error in IRI and cross correlation........................141

Figure C-5. Distribution of IRI disagreement, cross correlation 0.93-0.94................142

Figure C-6. IRI disagreement at various cross correlation levels.............................143

Figure C-7. IRI disagreement at high cross correlation levels..........................144

Figure C-8. IRI disagreement associated with cross correlation level, ultrasonic

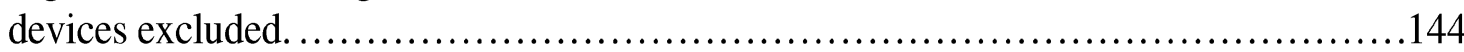




\section{LIST OF TABLES}

Table A-1. Sections measured in the 1993 RPUG experiment........................26

Table A-2. Devices that participated in the 1993 RPUG experiment. ...................27

Table A-3. Longitudinal distance measurement accuracy. ...........................29

Table A-4. Repeatability of profilers in measurement of IRI.........................32

Table A-5. Repeatability of profiler types in measurement of IRI. ......................33

Table A-6. Repeatability of profilers in measurement of RN.........................34

Table A-7. Agreement to Dipstick of profilers in measurement of IRI. .................36

Table A-8. Agreement to the Dipstick of profiler types in IRI measurement. .............38

Table A-9. Bias in profilers by section in Pennsylvania. .............................39

Table A-10. Bias in profilers by section in South Dakota....................................40

Table A-11. Speed sensitivity of profilers in Mississippi.............................41

Table A-12. Speed sensitivity of profilers in Nevada. ..............................4 4

Table A-13. Speed sensitivity of profilers in Pennsylvania...................................41

Table A-14. Speed sensitivity of profilers in South Dakota. .............................41

Table A-15. Summary of trends in MRI with operating speed.........................42

Table A-16. Summary of trends in RN with operating speed..........................43

Table B-1. Coverage of each site in the 1993 RPUG experiment............................47

Table B-2. Mississippi Site 1 Left, IRI Filter..................................48

Table B-3. Mississippi Site 1 Left, Long Wavelengths......................................48

Table B-4. Mississippi Site 1 Left, Medium Wavelengths. ............................48

Table B-5. Mississippi Site 1 Left, Short Wavelengths. ............................48

Table B-6. Mississippi Site 1 Right, IRI Filter...................................49

Table B-7. Mississippi Site 1 Right, Long Wavelengths..............................49

Table B-8. Mississippi Site 1 Right, Medium Wavelengths. ..........................49

Table B-9. Mississippi Site 1 Right, Short Wavelengths..................................49

Table B-10. Mississippi Site 2 Left, IRI Filter. .................................50

Table B-11. Mississippi Site 2 Left, Long Wavelengths...........................50

Table B-12. Mississippi Site 2 Left, Medium Wavelengths.................................50

Table B-13. Mississippi Site 2 Left, Short Wavelengths............................50

Table B-14. Mississippi Site 2 Right, IRI Filter...........................................51

Table B-15. Mississippi Site 2 Right, Long Wavelengths. ........................51

Table B-16. Mississippi Site 2 Right, Medium Wavelengths.........................51

Table B-17. Mississippi Site 2 Right, Short Wavelengths. ..........................51

Table B-18. Mississippi Site 3 Left, IRI Filter. ....................................52

Table B-19. Mississippi Site 3 Left, Long Wavelengths. ............................52

Table B-20. Mississippi Site 3 Left, Medium Wavelengths................................52

Table B-21. Mississippi Site 3 Left, Short Wavelengths..........................55

Table B-22. Mississippi Site 3 Right, IRI Filter..........................................53

Table B-23. Mississippi Site 3 Right, Long Wavelengths. ..........................53

Table B-24. Mississippi Site 3 Right, Medium Wavelengths.........................53

Table B-25. Mississippi Site 3 Right, Short Wavelengths..........................53

Table B-26. Mississippi Site 4 Left, IRI Filter. .................................54

Table B-27. Mississippi Site 4 Left, Long Wavelengths. .............................54

Table B-28. Mississippi Site 4 Left, Medium Wavelengths.................................54

Table B-29. Mississippi Site 4 Left, Short Wavelengths............................54 


\section{LIST OF TABLES}

Table B-30. Mississippi Site 4 Right, IRI Filter..............................................55

Table B-31. Mississippi Site 4 Right, Long Wavelengths. .............................55

Table B-32. Mississippi Site 4 Right, Medium Wavelengths...........................55

Table B-33. Mississippi Site 4 Right, Short Wavelengths..............................55

Table B-34. Mississippi Site 5 Left, IRI Filter. .......................................56

Table B-35. Mississippi Site 5 Left, Long Wavelengths...............................56

Table B-36. Mississippi Site 5 Left, Medium Wavelengths..................................56

Table B-37. Mississippi Site 5 Left, Short Wavelengths................................56

Table B-38. Mississippi Site 5 Right, IRI Filter...........................................57

Table B-39. Mississippi Site 5 Right, Long Wavelengths. .............................57

Table B-40. Mississippi Site 5 Right, Medium Wavelengths............................57

Table B-41. Mississippi Site 5 Right, Short Wavelengths.............................57

Table B-42. Mississippi Site 6 Left, IRI Filter. ........................................58

Table B-43. Mississippi Site 6 Left, Long Wavelengths...............................58

Table B-44. Mississippi Site 6 Left, Medium Wavelengths...................................58

Table B-45. Mississippi Site 6 Left, Short Wavelengths.................................58

Table B-46. Mississippi Site 6 Right, IRI Filter...........................................59

Table B-47. Mississippi Site 6 Right, Long Wavelengths. .............................59

Table B-48. Mississippi Site 6 Right, Medium Wavelengths............................59

Table B-49. Mississippi Site 6 Right, Short Wavelengths.............................59

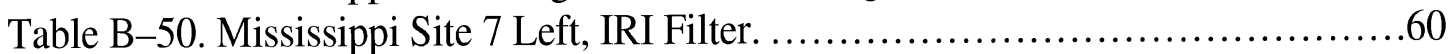

Table B-51. Mississippi Site 7 Left, Long Wavelengths................................60

Table B-52. Mississippi Site 7 Left, Medium Wavelengths..................................60

Table B-53. Mississippi Site 7 Left, Short Wavelengths..................................60

Table B-54. Mississippi Site 7 Right, IRI Filter...........................................61

Table B-55. Mississippi Site 7 Right, Long Wavelengths. ...............................61

Table B-56. Mississippi Site 7 Right, Medium Wavelengths............................61

Table B-57. Mississippi Site 7 Right, Short Wavelengths.............................61

Table B-58. Mississippi Site 8 Left, IRI Filter. ........................................62

Table B-59. Mississippi Site 8 Left, Long Wavelengths.................................62

Table B-60. Mississippi Site 8 Left, Medium Wavelengths...................................62

Table B-61. Mississippi Site 8 Left, Short Wavelengths..................................62

Table B-62. Mississippi Site 8 Right, IRI Filter............................................63

Table B-63. Mississippi Site 8 Right, Long Wavelengths. ...............................63

Table B-64. Mississippi Site 8 Right, Medium Wavelengths.............................63

Table B-65. Mississippi Site 8 Right, Short Wavelengths...............................63

Table B-66. Nevada Site 1 Left, IRI Filter..............................................64

Table B-67. Nevada Site 1 Left, Long Wavelengths...........................................64

Table B-68. Nevada Site 1 Left, Medium Wavelengths..................................64

Table B-69. Nevada Site 1 Left, Short Wavelengths. ....................................64

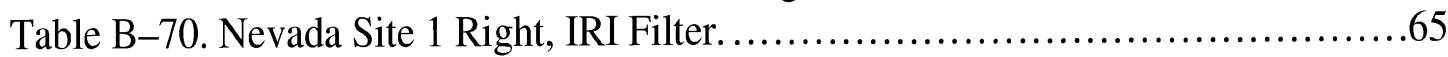

Table B-71. Nevada Site 1 Right, Long Wavelengths...................................65

Table B-72. Nevada Site 1 Right, Medium Wavelengths. .................................65

Table B-73. Nevada Site 1 Right, Short Wavelengths.........................................65

Table B-74. Nevada Site 2 Left, IRI Filter..............................................66 


\section{LIST OF TABLES}

Table B-75. Nevada Site 2 Left, Long Wavelengths..........................................66

Table B-76. Nevada Site 2 Left, Medium Wavelengths...................................66

Table B-77. Nevada Site 2 Left, Short Wavelengths. ...................................66

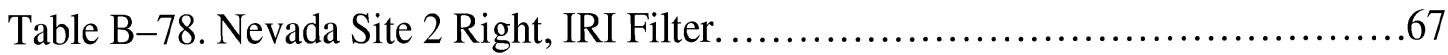

Table B-79. Nevada Site 2 Right, Long Wavelengths...................................67

Table B-80. Nevada Site 2 Right, Medium Wavelengths...............................67

Table B-81. Nevada Site 2 Right, Short Wavelengths........................................67

Table B-82. Nevada Site 3 Left, IRI Filter.............................................68

Table B-83. Nevada Site 3 Left, Long Wavelengths..........................................68

Table B-84. Nevada Site 3 Left, Medium Wavelengths....................................68

Table B-85. Nevada Site 3 Left, Short Wavelengths. ...................................68

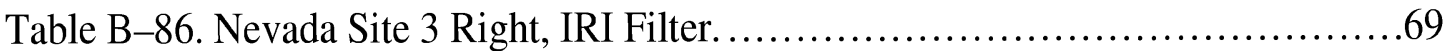

Table B-87. Nevada Site 3 Right, Long Wavelengths...................................69

Table B-88. Nevada Site 3 Right, Medium Wavelengths................................69

Table B-89. Nevada Site 3 Right, Short Wavelengths.........................................69

Table B-90. Nevada Site 4 Left, IRI Filter...............................................70

Table B-91. Nevada Site 4 Left, Long Wavelengths...........................................70

Table B-92. Nevada Site 4 Left, Medium Wavelengths..................................70

Table B-93. Nevada Site 4 Left, Short Wavelengths. .................................... 70

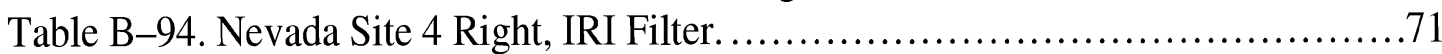

Table B-95. Nevada Site 4 Right, Long Wavelengths....................................

Table B-96. Nevada Site 4 Right, Medium Wavelengths................................

Table B-97. Nevada Site 4 Right, Short Wavelengths.........................................71

Table B-98. Nevada Site 5 Left, IRI Filter............................................72

Table B-99. Nevada Site 5 Left, Long Wavelengths.........................................72

Table B-100. Nevada Site 5 Left, Medium Wavelengths......................................72

Table B-101. Nevada Site 5 Left, Short Wavelengths..................................72

Table B-102. Nevada Site 5 Right, IRI Filter..............................................73

Table B-103. Nevada Site 5 Right, Long Wavelengths. ................................73

Table B-104. Nevada Site 5 Right, Medium Wavelengths................................73

Table B-105. Nevada Site 5 Right, Short Wavelengths...............................73

Table B-106. Nevada Site 6 Left, IRI Filter. ..............................................74

Table B-107. Nevada Site 6 Left, Long Wavelengths................................. 74

Table B-108. Nevada Site 6 Left, Medium Wavelengths.....................................74

Table B-109. Nevada Site 6 Left, Short Wavelengths....................................74

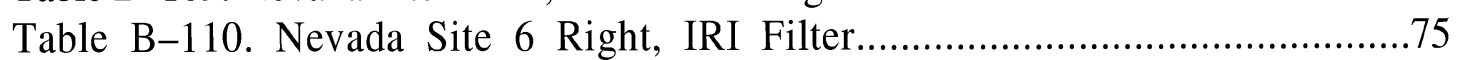

Table B-111. Nevada Site 6 Right, Long Wavelengths. ................................75

Table B-112. Nevada Site 6 Right, Medium Wavelengths..............................75

Table B-113. Nevada Site 6 Right, Short Wavelengths...............................75

Table B-114. Pennsylvania Site 1 Left, IRI Filter...........................................

Table B-115. Pennsylvania Site 1 Left, Long Wavelengths.............................76

Table B-116. Pennsylvania Site 1 Left, Medium Wavelengths..........................77

Table B-117. Pennsylvania Site 1 Left, Short Wavelengths.............................77

Table B-118. Pennsylvania Site 1 Right, IRI Filter...................................78

Table B-119. Pennsylvania Site 1 Right, Long Wavelengths..................................78 


\section{LIST OF TABLES}

Table B-120. Pennsylvania Site 1 Right, Medium Wavelengths..........................79

Table B-121. Pennsylvania Site 1 Right, Short Wavelengths...........................79

Table B-122. Pennsylvania Site 2 Left, IRI Filter..............................................80

Table B-123. Pennsylvania Site 2 Left, Long Wavelengths. ......................... 80

Table B-124. Pennsylvania Site 2 Left, Medium Wavelengths...........................81

Table B-125. Pennsylvania Site 2 Left, Short Wavelengths...............................

Table B-126. Pennsylvania Site 2 Right, IRI Filter.....................................

Table B-127. Pennsylvania Site 2 Right, Long Wavelengths.................................82

Table B-128. Pennsylvania Site 2 Right, Medium Wavelengths.........................83

Table B-129. Pennsylvania Site 2 Right, Short Wavelengths. .............................83

Table B-130. Pennsylvania Site 3 Left, IRI Filter.............................................84

Table B-131. Pennsylvania Site 3 Left, Long Wavelengths. ............................84

Table B-132. Pennsylvania Site 3 Left, Medium Wavelengths............................85

Table B-133. Pennsylvania Site 3 Left, Short Wavelengths.............................85

Table B-134. Pennsylvania Site 3 Right, IRI Filter.................................... 86

Table B-135. Pennsylvania Site 3 Right, Long Wavelengths..................................86

Table B-136. Pennsylvania Site 3 Right, Medium Wavelengths..........................87

Table B-137. Pennsylvania Site 3 Right, Short Wavelengths. ..........................87

Table B-138. Pennsylvania Site 4 Left, IRI Filter..............................................8

Table B-139. Pennsylvania Site 4 Left, Long Wavelengths. ..............................88

Table B-140. Pennsylvania Site 4 Left, Medium Wavelengths.............................89

Table B-141. Pennsylvania Site 4 Left, Short Wavelengths...............................89

Table B-142. Pennsylvania Site 4 Right, IRI Filter.....................................90

Table B-143. Pennsylvania Site 4 Right, Long Wavelengths.................................90

Table B-144. Pennsylvania Site 4 Right, Medium Wavelengths............................91

Table B-145. Pennsylvania Site 4 Right, Short Wavelengths. ............................91

Table B-146. Pennsylvania Site 5 Left, IRI Filter........................................92

Table B-147. Pennsylvania Site 5 Left, Long Wavelengths............................92

Table B-148. Pennsylvania Site 5 Left, Medium Wavelengths............................93

Table B-149. Pennsylvania Site 5 Left, Short Wavelengths.............................93

Table B-150. Pennsylvania Site 5 Right, IRI Filter.......................................94

Table B-151. Pennsylvania Site 5 Right, Long Wavelengths..................................94

Table B-152. Pennsylvania Site 5 Right, Medium Wavelengths.........................95

Table B-153. Pennsylvania Site 5 Right, Short Wavelengths. ........................95

Table B-154. Pennsylvania Site 6 Left, IRI Filter...........................................96

Table B-155. Pennsylvania Site 6 Left, Long Wavelengths. ...........................96

Table B-156. Pennsylvania Site 6 Left, Medium Wavelengths............................97

Table B-157. Pennsylvania Site 6 Left, Short Wavelengths..............................97

Table B-158. Pennsylvania Site 6 Right, IRI Filter....................................98

Table B-159. Pennsylvania Site 6 Right, Long Wavelengths................................98

Table B-160. Pennsylvania Site 6 Right, Medium Wavelengths.........................99

Table B-161. Pennsylvania Site 6 Right, Short Wavelengths. ...........................99

Table B-162. Pennsylvania Site 7 Left, IRI Filter...........................................100

Table B-163. Pennsylvania Site 7 Left, Long Wavelengths..............................100

Table B-164. Pennsylvania Site 7 Left, Medium Wavelengths............................101 


\section{LIST OF TABLES}

Table B-165. Pennsylvania Site 7 Left, Short Wavelengths..............................101

Table B-166. Pennsylvania Site 7 Right, IRI Filter.................................102

Table B-167. Pennsylvania Site 7 Right, Long Wavelengths..................................102

Table B-168. Pennsylvania Site 7 Right, Medium Wavelengths..........................103

Table B-169. Pennsylvania Site 7 Right, Short Wavelengths. ..........................103

Table B-170. Pennsylvania Site 8 Left, IRI Filter..............................................104

Table B-171. Pennsylvania Site 8 Left, Long Wavelengths.............................104

Table B-172. Pennsylvania Site 8 Left, Medium Wavelengths...........................105

Table B-173. Pennsylvania Site 8 Left, Short Wavelengths............................105

Table B-174. Pennsylvania Site 8 Right, IRI Filter.................................106

Table B-175. Pennsylvania Site 8 Right, Long Wavelengths..................................106

Table B-176. Pennsylvania Site 8 Right, Medium Wavelengths.........................107

Table B-177. Pennsylvania Site 8 Right, Short Wavelengths. ..........................107

Table B-178. South Dakota Site 1 Left, IRI Filter. ...................................108

Table B-179. South Dakota Site 1 Left, Long Wavelengths............................108

Table B-180. South Dakota Site 1 Left, Medium Wavelengths................................109

Table B-181. South Dakota Site 1 Left, Short Wavelengths.............................109

Table B-182. South Dakota Site 1 Right, IRI Filter..........................................110

Table B-183. South Dakota Site 1 Right, Long Wavelengths. ...........................110

Table B-184. South Dakota Site 1 Right, Medium Wavelengths.........................110

Table B-185. South Dakota Site 1 Right, Short Wavelengths...........................110

Table B-186. South Dakota Site 2 Left, IRI Filter. ....................................111

Table B-187. South Dakota Site 2 Left, Long Wavelengths.............................111

Table B-188. South Dakota Site 2 Left, Medium Wavelengths..............................112

Table B-189. South Dakota Site 2 Left, Short Wavelengths.............................112

Table B-190. South Dakota Site 2 Right, IRI Filter........................................113

Table B-191. South Dakota Site 2 Right, Long Wavelengths. ..........................113

Table B-192. South Dakota Site 2 Right, Medium Wavelengths..........................113

Table B-193. South Dakota Site 2 Right, Short Wavelengths...........................113

Table B-194. South Dakota Site 3 Left, IRI Filter. .....................................114

Table B-195. South Dakota Site 3 Left, Long Wavelengths.............................114

Table B-196. South Dakota Site 3 Left, Medium Wavelengths.................................115

Table B-197. South Dakota Site 3 Left, Short Wavelengths............................115

Table B-198. South Dakota Site 3 Right, IRI Filter..........................................116

Table B-199. South Dakota Site 3 Right, Long Wavelengths. ..........................116

Table B-200. South Dakota Site 3 Right, Medium Wavelengths........................116

Table B-201. South Dakota Site 3 Right, Short Wavelengths...........................116

Table B-202. South Dakota Site 4 Left, IRI Filter. ......................................117

Table B-203. South Dakota Site 4 Left, Long Wavelengths............................117

Table B-204. South Dakota Site 4 Left, Medium Wavelengths................................118

Table B-205. South Dakota Site 4 Left, Short Wavelengths.............................118

Table B-206. South Dakota Site 4 Right, IRI Filter.........................................119

Table B-207. South Dakota Site 4 Right, Long Wavelengths. .........................119

Table B-208. South Dakota Site 4 Right, Medium Wavelengths..........................119

Table B-209. South Dakota Site 4 Right, Short Wavelengths............................119 


\section{LIST OF TABLES}

Table B-210. South Dakota Site 5 Left, IRI Filter. ..................................120

Table B-211. South Dakota Site 5 Left, Long Wavelengths............................120

Table B-212. South Dakota Site 5 Left, Medium Wavelengths...............................121

Table B-213. South Dakota Site 5 Left, Short Wavelengths.............................121

Table B-214. South Dakota Site 5 Right, IRI Filter...........................................122

Table B-215. South Dakota Site 5 Right, Long Wavelengths..........................122

Table B-216. South Dakota Site 5 Right, Medium Wavelengths........................122

Table B-217. South Dakota Site 5 Right, Short Wavelengths.........................122

Table B-218. South Dakota Site 6 Left, IRI Filter. ..................................123

Table B-219. South Dakota Site 6 Left, Long Wavelengths............................123

Table B-220. South Dakota Site 6 Left, Medium Wavelengths...............................124

Table B-221. South Dakota Site 6 Left, Short Wavelengths............................124

Table B-222. South Dakota Site 6 Right, IRI Filter..........................................125

Table B-223. South Dakota Site 6 Right, Long Wavelengths. ..........................125

Table B-224. South Dakota Site 6 Right, Medium Wavelengths........................125

Table B-225. South Dakota Site 6 Right, Short Wavelengths..........................125

Table B-226. South Dakota Site 7 Left, IRI Filter. ..................................126

Table B-227. South Dakota Site 7 Left, Long Wavelengths...........................126

Table B-228. South Dakota Site 7 Left, Medium Wavelengths................................127

Table B-229. South Dakota Site 7 Left, Short Wavelengths...........................127

Table B-230. South Dakota Site 7 Right, IRI Filter..........................................128

Table B-231. South Dakota Site 7 Right, Long Wavelengths. ..........................128

Table B-232. South Dakota Site 7 Right, Medium Wavelengths.......................128

Table B-233. South Dakota Site 7 Right, Short Wavelengths..........................128

Table B-234. South Dakota Site 8 Left, IRI Filter. ....................................129

Table B-235. South Dakota Site 8 Left, Long Wavelengths.............................129

Table B-236. South Dakota Site 8 Left, Medium Wavelengths................................130

Table B-237. South Dakota Site 8 Left, Short Wavelengths............................130

Table B-238. South Dakota Site 8 Right, IRI Filter..........................................131

Table B-239. South Dakota Site 8 Right, Long Wavelengths. ...........................131

Table B-240. South Dakota Site 8 Right, Medium Wavelengths.........................131

Table B-241. South Dakota Site 8 Right, Short Wavelengths............................131

Table C-1. Repeatability of profilers in measurement of IRI, left side.......................134

Table C-2. Repeatability of profilers in measurement of IRI, right side..................135

Table C-3. Agreement to Dipstick in measurement of IRI, left.........................139

Table C-4. Agreement to Dipstick in measurement of IRI, right. ........................140

Table C-5. Expected IRI error associated with cross correlation level...................145

Table C-6. Expected IRI error associated with cross correlation level, ultrasonic

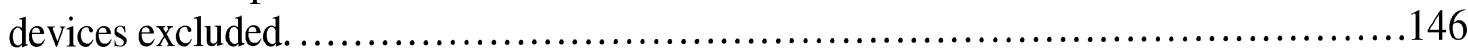




\section{INTRODUCTION}

Standard methods of comparing two profile measurements usually place tolerances on agreement between elevation values over a broad waveband. A weakness of this approach is that it fails to emphasize the aspects of profile measurement that are more relevant to the intended application of the device. The ratings furnished by these methods for agreement between profiles does not have a direct relationship to the agreement that can be expected in summary index values. The most common method of objective profile comparison, American Society for Testing and Materials (ASTM) E-950, does not motivate a situation in which two "certified" profilers are expected to measure the same value of International Roughness Index (IRI) within a well-defined, and sufficiently tight, tolerance. When ASTM E-950 is used in profiler certification programs for construction quality control, two certified profilers often go on to produce IRI values in practice for the same site that imply differing levels of smoothness incentive pay.

This report presents an objective procedure for rating the agreement between profile measurements. The procedure is based on the cross correlation function, described by Bendat and Piersol. (1) A method of cross correlating profile measurements is proposed which:

- rates agreement between profiles in a given waveband, including that of a roughness index of interest,

- deduces the longitudinal distance offset between two profile measurements and compensates for it,

- searches for the linear distance measurement error in a profile measurement, and

- provides a single unitless rating of agreement ranging from -1 to 1 that may be specified in a test method.

The rating of agreement provided by this procedure represents repeatability when it is applied to two measurements of the same profile by the same device. It represents reproducibility when it is applied to two measurements of the same profile by different devices, and it represents accuracy when a measurement from one of those devices is deemed to be correct.

This method is intended for application in profiler comparison studies as well as profiler certification testing programs. The method can also be used to classify profilers for individual applications when threshold correlation values are developed. Cross correlation is superior to direct comparison of index values because it compares the overall roughness and its spatial distribution. The method yields ratings of agreement under a given set of test conditions that do not reward compensating error. Further, the method may be customized to a given application, such as measurement of a specific roughness index, or measurement of profile within a given waveband. When a correlation level is assigned to the measurement of an index, such as the IRI, it suggests a reasonable expectation of the same performance on sites of similar roughness and surface texture. This report relates the level of cross correlation for IRI output to the expected tolerance in IRI measurement, and shows how this may be done for other indices. 
This report describes alternative methods already used in practice and in the classical signal analysis literature, and proposes cross correlation as an alternative. The report presents the specifics of the cross correlation method and the steps used in applying it, provides an example of the application of the method in an attempt to relate correlation levels to expected profiler performance, and describes a testing program that would help finalize the method.

\section{EXISTING METHODS}

This section presents two methods that are already in use for rating the agreement between profile measurements: (1) ASTM E-950, and (2) inspection of the gain relationship. ASTM E-950 evaluates profiler accuracy and repeatability, but it is a poor choice for either purpose.

The "gain" method verifies a profiler by comparing its measurements to a reference profile that is deemed correct. This method is a good alternative for rating profile accuracy, and would require only minor adaptation for use in rating of repeatability. Expanding the technique to include the phase relationship and coherence would provide tremendous diagnostic information for all profile comparisons. The only drawback is the complexity in setting accuracy thresholds that are based on inspection of plots, rather than a few numerical values.

\section{ASTM E-950}

The ASTM Standard E-950 is currently the most widely used method for rating the repeatability and accuracy of profilers. (2) The Standard includes a classification system for profilers that is based on a composite level of precision among repeat elevation measurements and a composite level of bias (or the lack thereof) in elevation compared to a reference measurement. The composite values are based on a minimum of 10 profile measurements. The individual elevation measurements are compared over a distance of 321.9 meters (1056 feet) at 0.3 -meter (1-foot) intervals.

The main weakness of this approach is the emphasis on long wavelength content that results from comparison of elevation values. In most road profiles, the amplitude of elevation content is roughly proportional to wavelength. Thus, short wavelength features often appear as relatively small deviations in elevation. Comparison of elevation values, and treatment of each elevation value as a distinct measurement instead of part of the overall signal, prevents the Standard from detecting short wavelength measurement problems. The emphasis on long wavelength content also places a premium on the specific characteristics of the high-pass filter used in the profile computation. This is unfortunate, because the very long wavelength content is not of interest in most applications.

\section{Precision}

The precision of elevation measurement at a given point is rated by ASTM E-950 using the standard deviation of all elevation measurements at that location. The composite precision level over the entire profile is the average of all standard deviation values. A major weakness of this approach is the placement of a tolerance on elevation that is the 
same over the entire wavelength range. Road profiles, when treated as random signals, are known to commonly have elevation spectral density that decreases very rapidly with wavelength. (3) Performing analyses on profile elevation over a broad range of wavelengths biases the results by assigning disproportionate weight to the long wavelength content. (4) Short wavelength content in a profile may be significant to important road qualities, even at a low amplitude. This is because the reversals between upward and downward slope occur more quickly at shorter wavelengths, so a lower amplitude is needed to cause the same level of acceleration in a vehicle. The consequence of placing precision limits on elevation values over a broad waveband, therefore, is that short wavelength content may exhibit an unacceptable level of error with little penalty to the precision level.

Consider the influence profile elevation errors may have on the IRI. The wavelength response of the IRI is often characterized by the plot in figure 1. This plot provides the gain for profile slope. The response of the IRI is of the same order of magnitude for wavelengths ranging from about 1.2 to 30 meters. Errors in profile with roughly equal slope amplitudes are expected to have a similar impact on the IRI in this range.

The gain for profile elevation is shown in figure 2. This plot demonstrates that the IRI responds most heavily to elevation for wavelengths from 1.5 to 3 meters. Precision limits under ASTM E-950 are set on elevation over a broad waveband. Therefore, accurate IRI measurement may require precision limits that are unnecessarily restrictive for wavelength content below 1.5 meters and above 3 meters to ensure the needed precision in the band from 1.5 to 3 meters. Worse yet, the threshold values may have to be insufficient in the 1.5 to 3 meter range so that equipment can pass in the long wavelength range.

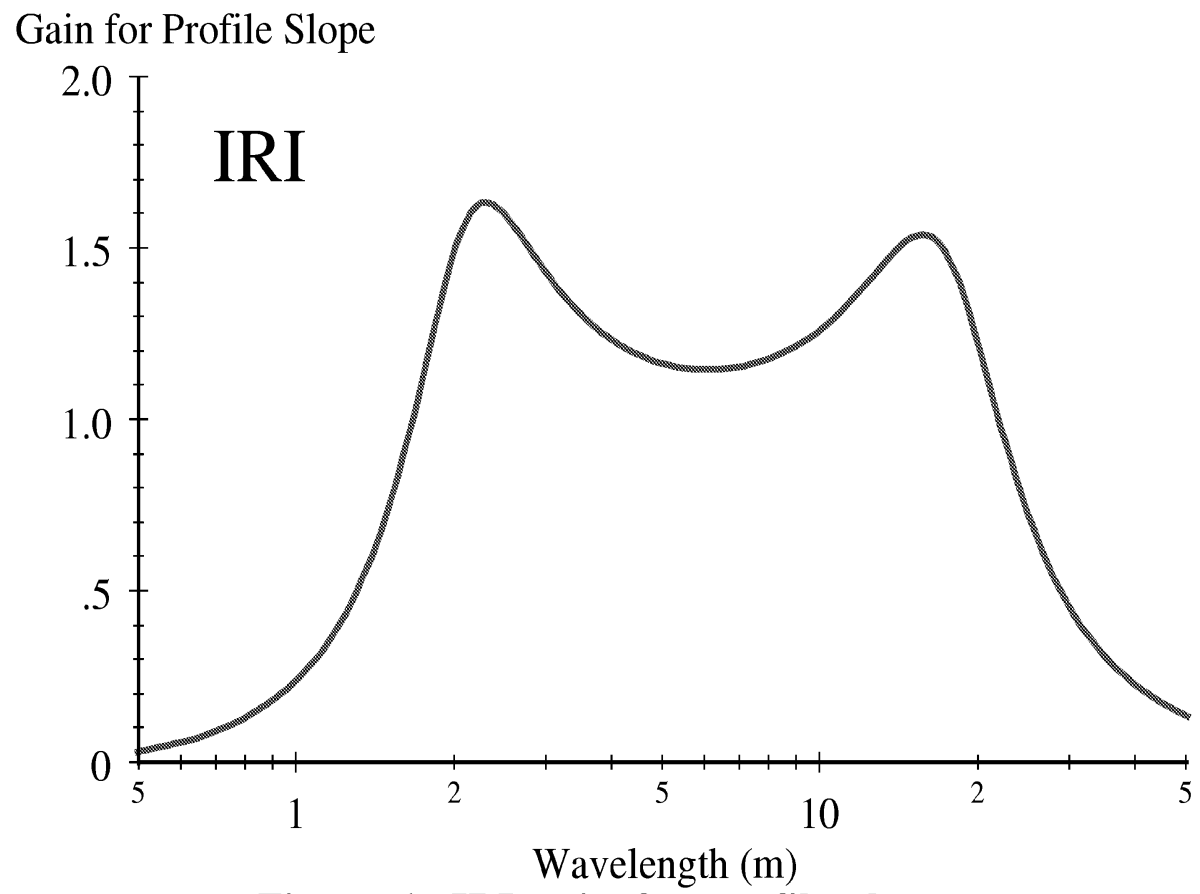

Figure 1. IRI gain for profile slope. 


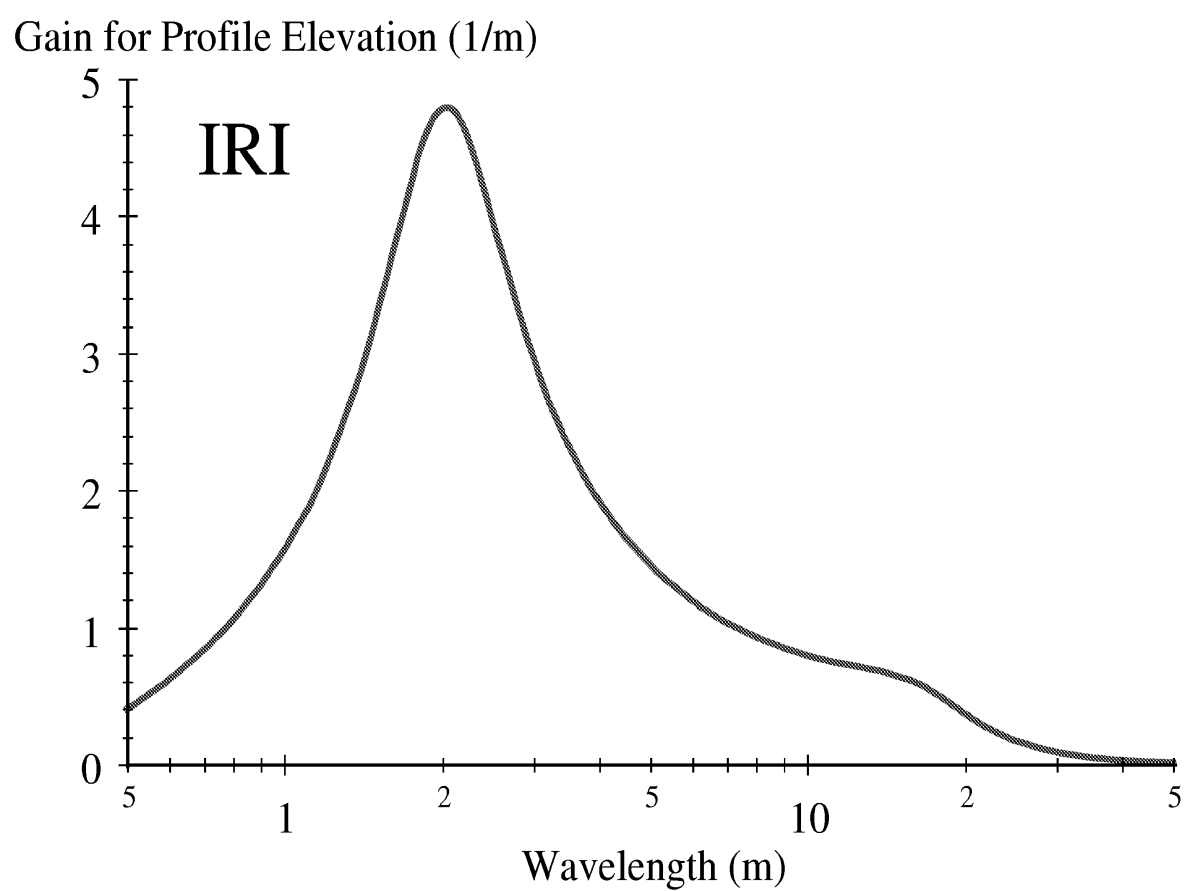

Figure 2. IRI gain for profile elevation.

This can be investigated by calculating the error level in IRI that could be imposed by sinusoidal noise without violating the precision limits specified in ASTM E-950. The Standard specifies a standard deviation limit of 0.38 millimeters for Class I equipment and 0.76 millimeters for Class II equipment. If a profile measurement of a perfectly flat road includes a sinusoidal error with a wavelength of 1 , an amplitude of $A$, and a phase lag of $f$, the profile $\mathrm{P}$ as a function of longitudinal distance $\mathrm{x}$ will be:

$$
\mathrm{P}(\mathrm{x})=\mathrm{A} \cdot \sin \left(\frac{2 \pi \mathrm{x}}{\lambda}+\phi\right)
$$

Over a long distance, this profile has an average rectified slope value of:

$$
\mathrm{ARS}=\frac{4 \mathrm{~A}}{\lambda}
$$

If the values of phase lag in repeat measurements are evenly distributed over the possible range, the values of amplitude (A) that correspond to the thresholds for Classes I and II are 0.54 millimeters and 1.07 millimeters, respectively, regardless of wavelength. An estimate of the error in IRI caused by the sinusoidal noise can be calculated by applying the gain at the appropriate wavelength. ${ }^{1}$ For example, the IRI algorithm has a gain for profile slope of about 1.63 at a wavelength of 2.32 meters. Using the precision threshold for Class I, the IRI error could be $1.52 \mathrm{~m} / \mathrm{km}$ :

$$
\text { IRI Error }=\text { IRI Gain } \bullet \text { ARS }=1.63 \bullet 4 \bullet(0.54 \mathrm{~mm}) /(2.32 \mathrm{~m}) .
$$

Figure 3 shows the theoretical error level that could exist as a function of wavelength for both precision levels. Each point in the plot is error in IRI that would be caused in the

\footnotetext{
1 This is only an estimate, because it does not include the influence of filter initialization.
} 
measurement of a perfectly flat road by a sinusoidal noise that does not violate the precision limit on elevation. Some of these error levels are quite extreme. Of course, profile measurement errors rarely appear as sinusoids with random phase shift. In addition, superimposing these sinusoidal errors on a non-zero profile would lead to compensating error, because not all portions of the error component would increase roughness.

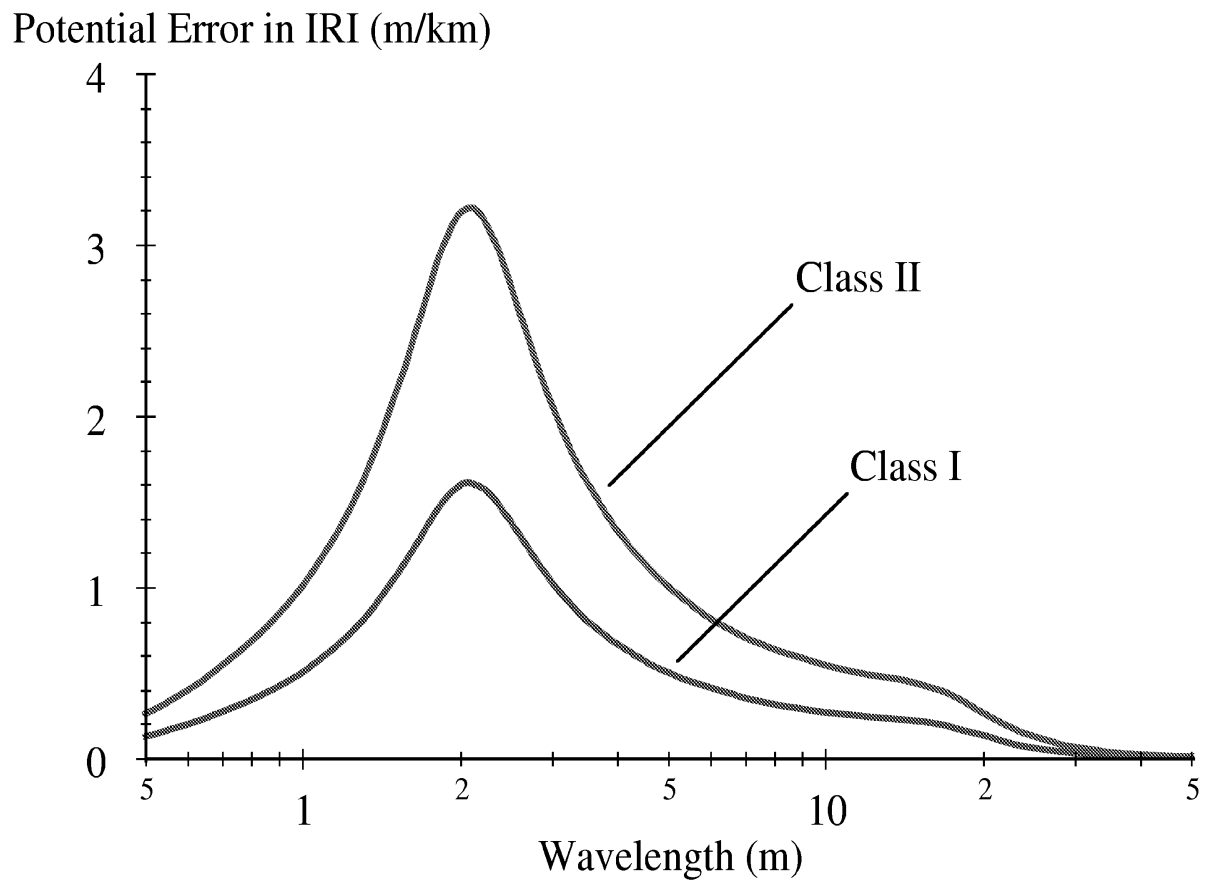

Figure 3. Theoretical error in IRI versus wavelength at Class I and II.

Profile measurement errors are much more likely to appear as random noise. This would lead to a much lower level of absolute IRI error at the same precision threshold. However, if the random noise exists over a broad range of wavelengths, figure 3 shows the relative error level that would be caused by each portion of its content. Overall, the figure demonstrates that ASTM E-950 does not protect against the effect on errors in the wavelength range from 1.5 to 3.0 meters on IRI. Profile measurement errors do occur that will appear in this range, such as incomplete correction of axle hop motion by the accelerometer, drift of a narrow laser footprint into and out of the trough of longitudinal tines, and electrical noise.

Although this example focused on measurement of IRI, the same effect will exist for other applications. This is a consequence of placing limits on the precision of elevation measurement. In any application, errors that affect the measurement of the short wavelength portion of the range of interest will always be ignored in favor of errors that affect the long wavelength portion.

\section{Bias}

In ASTM E-950, the bias in elevation measurement at a given point is rated by averaging the elevation values at that point by all repeat measurements and comparing it to the elevation value of the reference profile at that point. The difference between the two is the bias level. The composite bias level over the entire profile is the average of all bias 
values. The Standard specifies an average bias limit of 1.25 millimeters for Class I equipment and 2.50 millimeters for Class II equipment.

Several theoretical cases can be imagined in which limiting the bias as defined above fails to emphasize the proper aspects of agreement between profile measurements. First, ASTM E-950 does not specify a method of eliminating vertical offset between profiles, yet only rare applications of profile measurement are concerned with absolute elevation. A simple vertical offset between otherwise equivalent profiles will appear as a bias. Second, summing of individual bias values rewards compensating error when some values are high compared to the reference and some are low. Many profiles that have been low-pass filtered to exclude wavelengths over 91.4 meters, as specified in the Standard, have a average elevation value under 1.25 millimeters. It is therefore possible to qualify as a Class I instrument by reporting a profile of all zeros. A solution to this problem would be the replacement of individual bias values with their absolute values. (5) A thorough discussion of these and other statistical weaknesses of ASTM E-950 is provided by Li. (6) Third, like the precision criteria discussed above, the bias criteria places too much emphasis on long wavelength content and may ignore critical levels of error in the measurement of short wavelength features.

ASTM E-950 specifies that the amplitude and phase relationship of a profile measurement should be unaffected for wavelengths up to 60 meters. Most profilers apply a low-pass filter with a cutoff wavelength of about 90 meters that is designed to avoid modifying wavelength content below 60 meters. Not all of the this range is needed in most applications. (4) (Wavelengths near 60 meters affect the IRI very little.) As described above, comparison of elevation values places a premium on long wavelength content. Like the precision criteria, the bias criteria are unnecessarily sensitive to long wavelengths. The consequence of this is an undesirable sensitivity to the type of low-pass filter used in the profile computation.

In addition to placing unnecessary emphasis on long wavelength content, the bias criteria completely ignore short wavelength measurement errors. Consider the profile measurement shown in figure 4. This profile is artificially generated white noise slope, which is a rough approximation of the spectral characteristics of common profiles. The level of white noise gives this profile an IRI value of about $1.82 \mathrm{~m} / \mathrm{km}$. For this example, it will be treated as a reference measurement.

If a profiler reproduced this measurement perfectly, but applied a moving average to smooth the measurement before the bias calculation, some of the short wavelength content would be missing. (4) (The precise amount of content that is removed depends on the baselength of the average.) Since short wavelength features usually have low elevation amplitudes, they appear in the elevation trace as chatter but do not contribute to the larger fluctuations. Thus, applying the moving average may cause only a small absolute bias level. For example, when the profile is smoothed using a moving average with a baselength of 3 meters the trace in figure 5 is produced. The average absolute bias level of this smoothed profile, when compared to the original, is only 0.59 millimeters. This is well below the Class I limit. This implies that wavelengths under 3 meters need not even be included in the measurement to pass the bias criteria. Note that the smoothing reduced the overall IRI value to $0.71 \mathrm{~m} / \mathrm{km}$. 


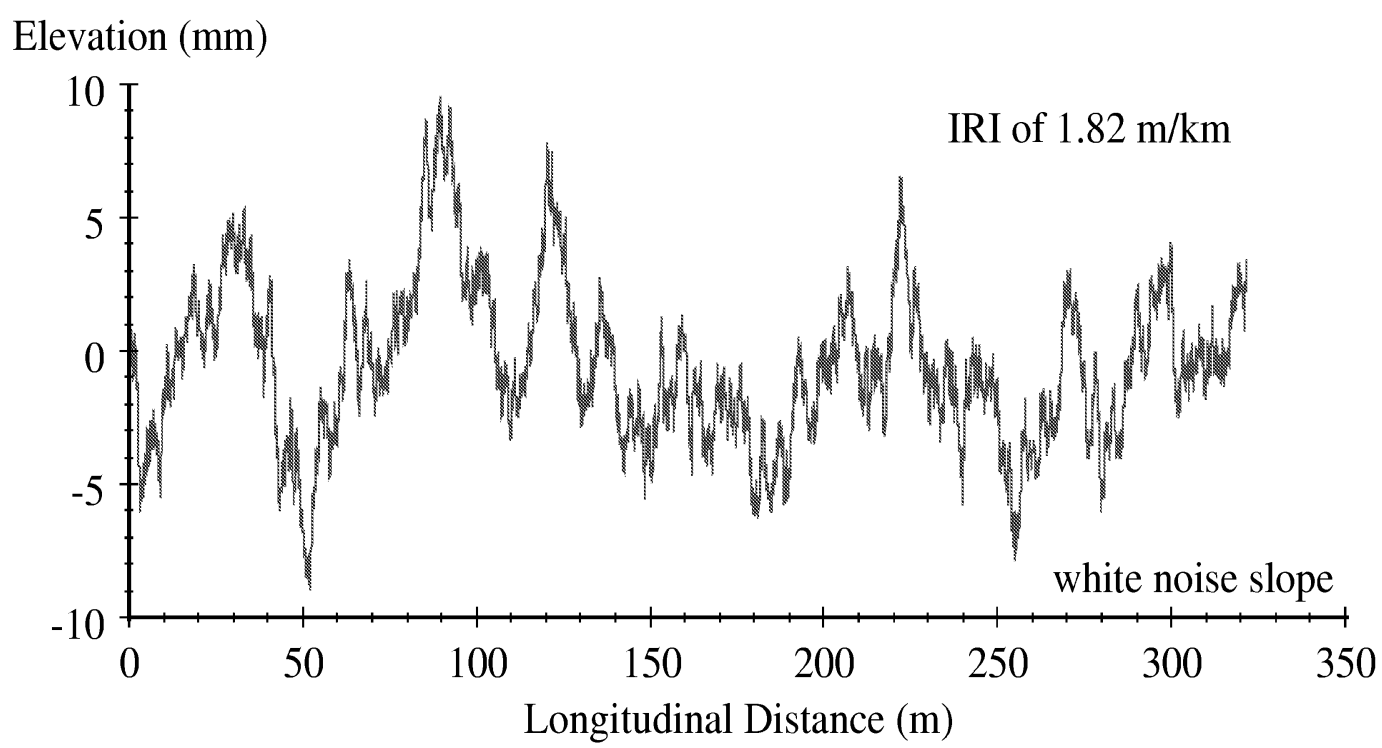

Figure 4. White noise slope profile.

Elevation (mm)

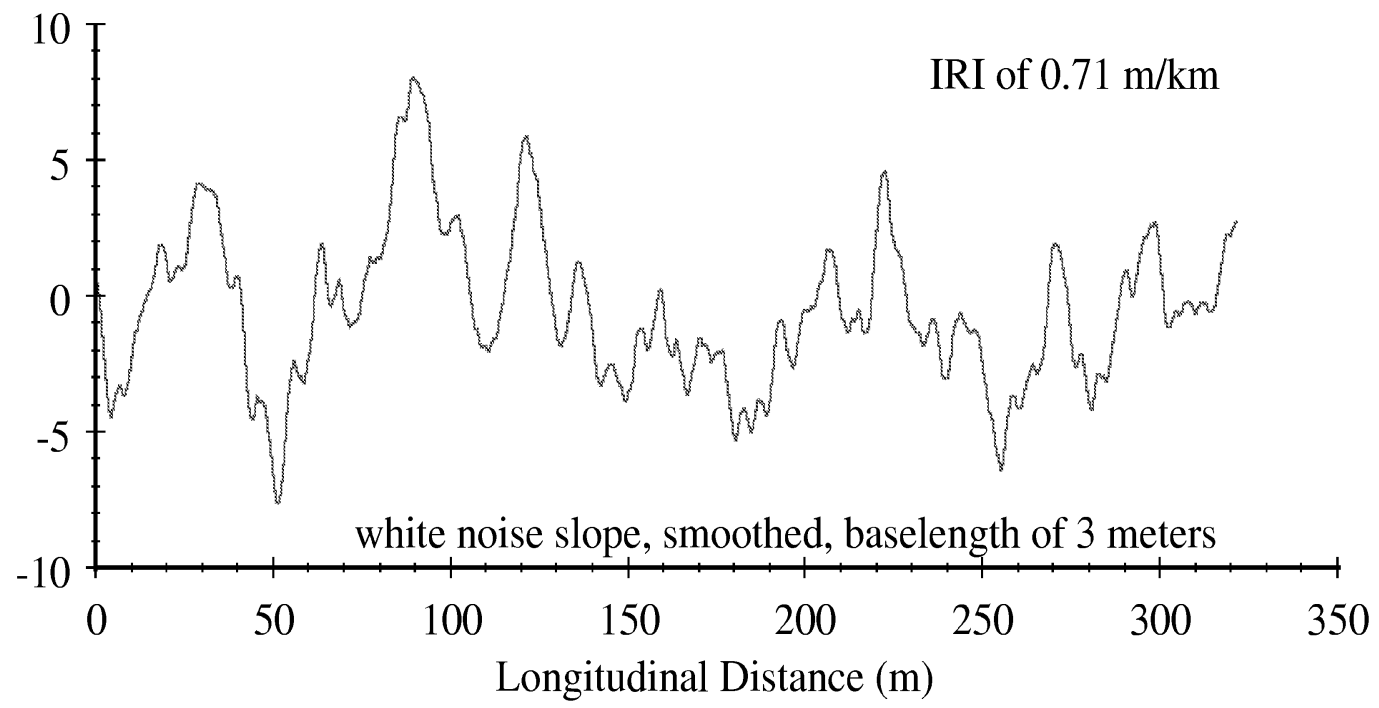

Figure 5. Profile smoothed with a 3-meter moving average.

Figures 6 and 7 show the results when this example is repeated over a range of moving average baselength values. Figure 6 shows the variation in average absolute bias that occurs as the baselength of the moving average is increased. The baselength must be increased to over 16 meters before the bias limit is violated. With a baselength of 16 meters, much of the wavelength range of interest for the IRI is removed, and most of the range of interest for the Ride Number (RN) and Michigan Ride Quality Index (RQI) is removed. This demonstrates the indifference of the ASTM E-950 bias criteria to errors or even omissions in short wavelength measurement. Figure 7 shows the variation in IRI with baselength. Note that the moving average baselength can be increased so far without violating the bias limit that a profile with an IRI of under $0.15 \mathrm{~m} / \mathrm{km}$ would pass.

A significant amount of the wavelength range can also be removed before the Class I precision limit is violated. The standard deviation of "error" in elevation does not increase 
above 0.38 millimeters (the Class I limit) until the low-pass filter cutoff wavelength is increased to 2.75 meters. A low-pass filter with this cutoff reduces the IRI to $0.82 \mathrm{~m} / \mathrm{km}$.

Of course, the precise amount of the wavelength range ignored by the bias and precision criteria for a Class I device depends on the specific wavelength content of the reference profile and the level of genuine error that exists in the test measurements. Nevertheless, some significant portion of the short wavelength range of interest is not sufficiently captured by ASTM E-950.

Average Absolute Bias (mm)

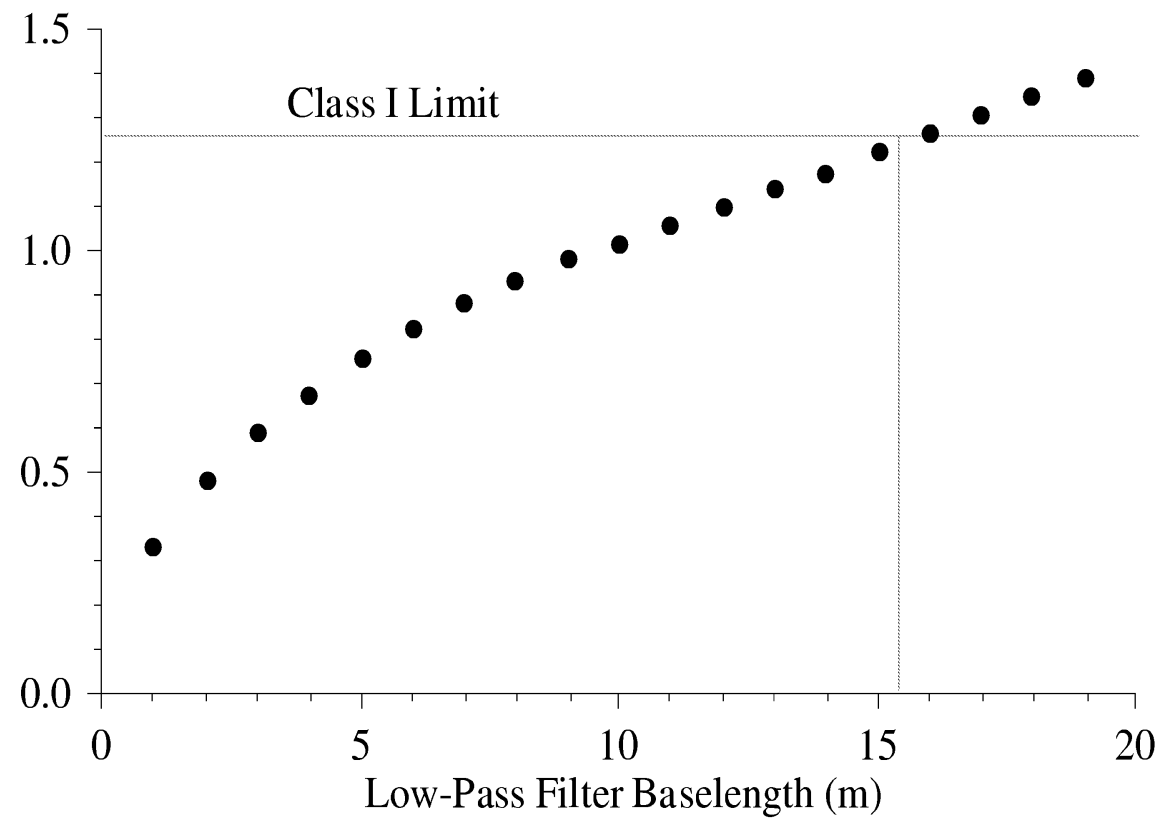

Figure 6. Absolute bias level of smoothed profiles.

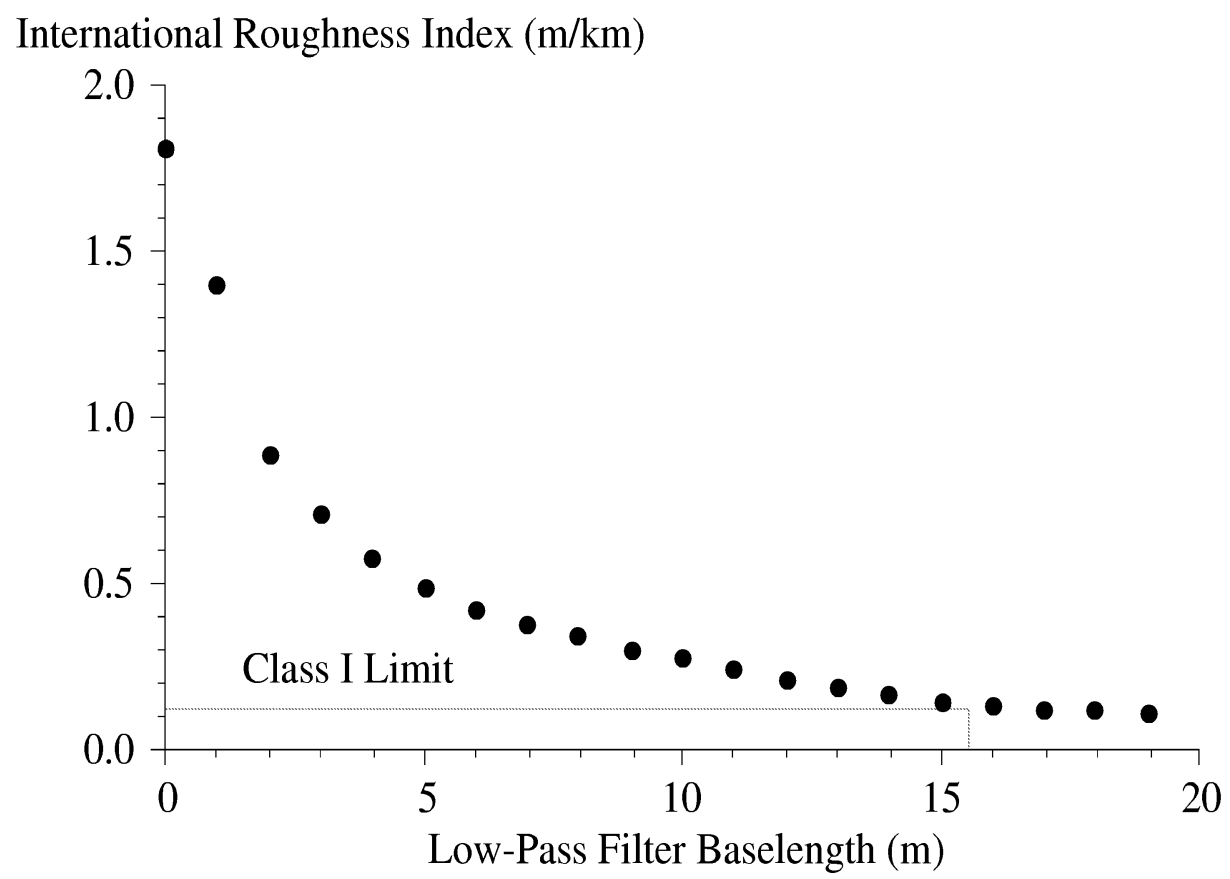

Figure 7. IRI of smoothed profiles. 


\section{Profiler Gain Limits}

Hans Prem developed a method of validating pavement profile measurements using the transfer function between a reference profile and profiles collected by a (candidate) device under evaluation. (7) In this method, the reference profile measurement is treated as the input, and each repeat profile measurement by the candidate device is treated as output with a linear relationship to the reference profile. For each repeat measurement, a transfer function is calculated. (Strictly speaking, this is only a transfer function if the reference measurement is truly correct, and the input-output relationship is then between the actual profile of the road and the candidate profiler's output.) The transfer function gain values at each wavelength (or wave number) are then averaged across the set of repeats. Limits are placed on the composite transfer function that represent expected error limits in IRI. Figure 8 provides an example.

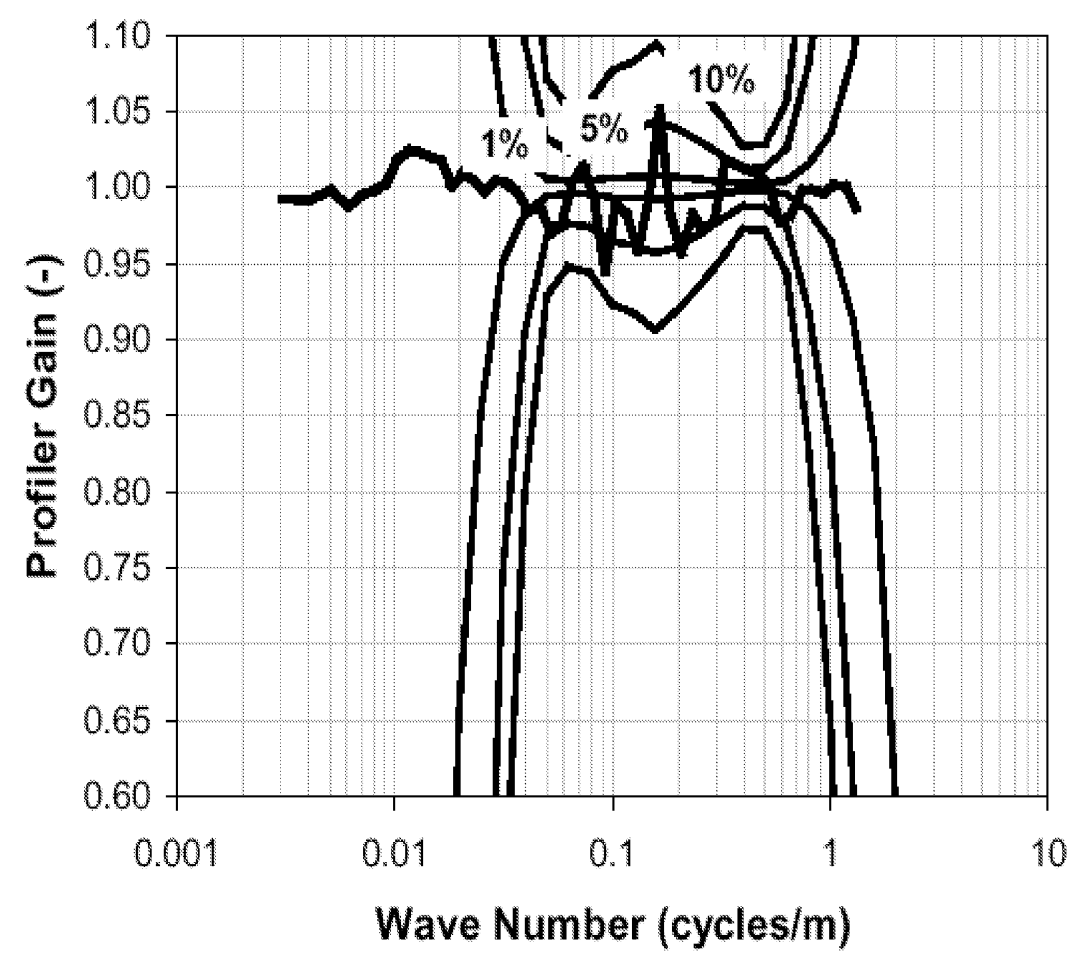

Figure 8. Profile comparison using transfer functions, after Prem. (8)

This approach has several advantages:

- The error limits can be customized for any index of interest, so only the relevant waveband is emphasized.

- This method may succeed with fewer measurement sites than simple comparison of summary index values. This is because profilers that may produce the same index value because of compensating error in the overall index value are not likely to produce acceptable transfer functions.

- The gain plot may provide diagnostic information about the source of error, particularly if the measurement error is confined to a narrow band or one end of the range of interest. 
These advantages make the specification of profiler gain limits a useful tool for validation of profilers for pavement network evaluation, or any other application where a profiler must produce accurate index values on a lot by lot basis.

To more completely define the relationship between the reference profile and a candidate profile measurement, the phase relationship could also be examined. This would help validate the spatial distribution of roughness, which may be important if the profiler must be verified for construction quality control.

Further definition of the relationship between profiles may also be needed if the method is ever extended to examination of repeatability. This is because repeat measurements by the same profiler often consistently possess the same level and type of noise. In this case, an acceptable transfer function may be obtained despite the presence of measurement errors. With this in mind, the gain criteria should be supplemented with a specification on coherence.

The coherence function provides an assessment of the relationship between two signals at each wavelength. A high level of coherence indicates that the relationship is linear and dependent. Coherence has a maximum value of unity for a perfect relationship and degrades below unity if any noise is present. The coherence function is also penalized if any content exists in the candidate profile measurement from a source other than the actual profile. A minimum level of coherence at a given wave number between the candidate profile and the reference measurement would ensure a systematic linear relationship between them, but not a gain of one. Therefore, a rigorous specification must require a minimum level of coherence and a gain near one over the relevant waveband.

Figure 9 shows the coherence between a measurement from a lightweight profiler and a slow-speed reference measurement on a segment of moderately-rough asphalt. These measurements only appear to have a strong relationship for wavelengths over about 10 meters. (Wavelength is the inverse of wave number, so a wavelength of 10 meters corresponds to a wave number of $0.1 \mathrm{cycles} / \mathrm{m}$.) The figure also shows the coherence between two repeat measurements by the lightweight profiler. These two measurements have a much stronger relationship for wavelengths shorter than 10 meters, but it does drop off below 1.5 meters.

Figure 10 shows the gain between the same pairs of measurements examined in figure 9. The comparison to the reference profile that produced such poor coherence also resulted in a poor comparison of spectral density, and thus, a poor gain characteristic. Through most of the wavelength range, the gain is less than one. In this analysis, the reference measurement is treated as the input and the measurement by the lightweight profiler is treated as the output. Thus, a gain of less than one indicates a lower level of roughness in the measurement by the lightweight. In fact, the IRI value produced by the lightweight was about $2.27 \mathrm{~m} / \mathrm{km}$ and the value produced by the reference device was about $2.43 \mathrm{~m} / \mathrm{km}$.

The gain between the two repeat measurements by the lightweight profiler has a value near one for the entire range. As expected, the IRI values match very closely. They differ by less than $0.01 \mathrm{~m} / \mathrm{km}$. (This gain characteristic is not nearly as close to one as the example displayed in figure 8 . This is partly because figure 8 shows the average of 10 individual gain characteristics.) Although the gain is very close to one for short 
wavelengths, the coherence function is not. This indicates that the roughness in this waveband is equal because of compensating error.

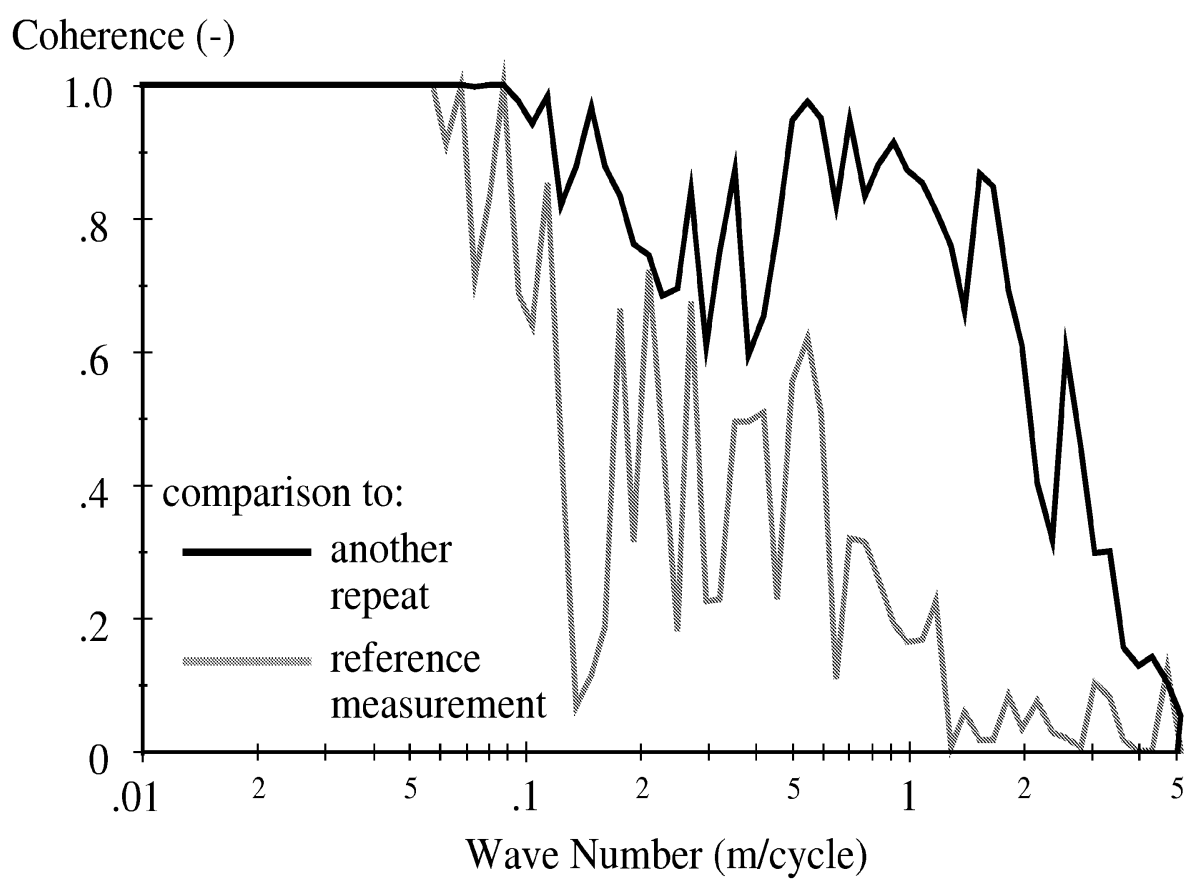

Figure 9. Coherence between road profiles.

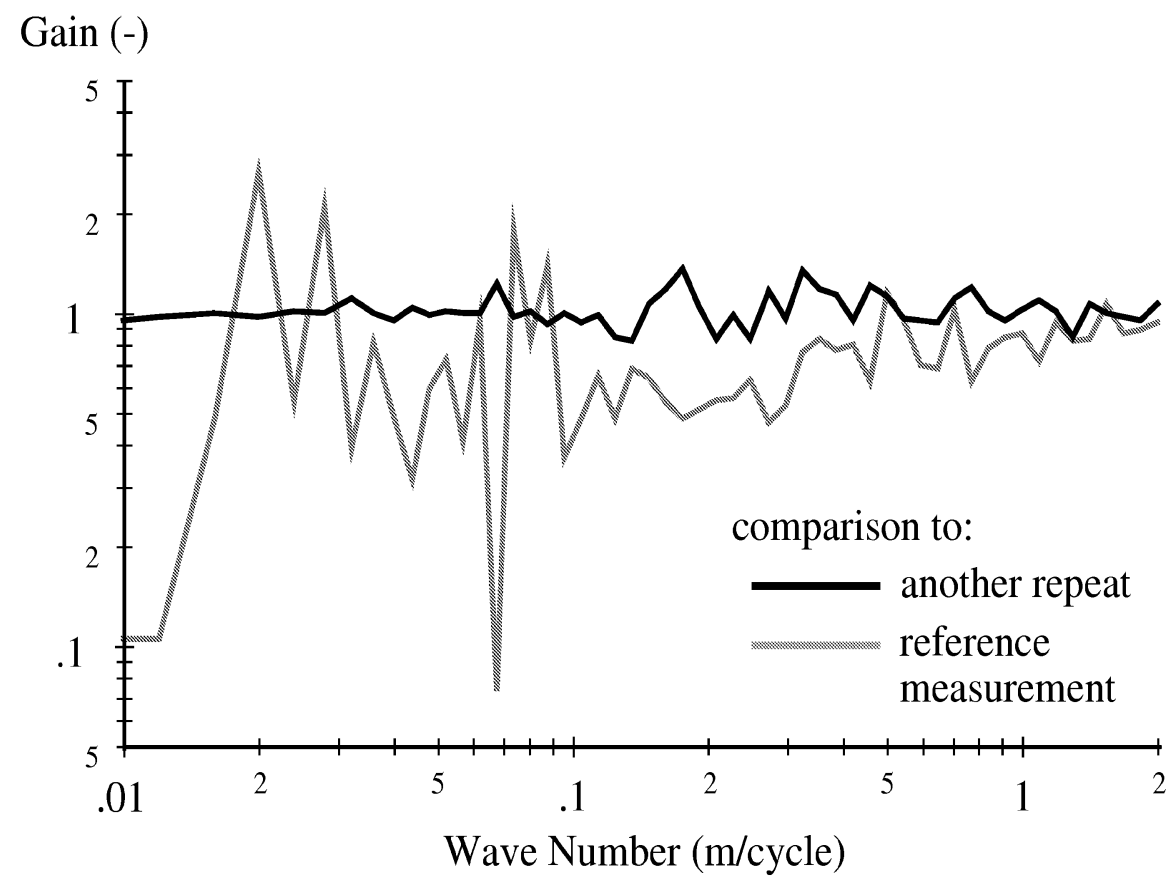

Figure 10. Gain between road profiles. 


\section{Cross CORRELATion}

This section describes the use of cross correlation for rating the agreement between profiles. Some of this material is adapted from a recent report on road profile interpretation. (4) This method is intended for rating of repeatability, reproducibility, or accuracy of profiles. It is based on the cross correlation function described by Bendat and Piersol for measurement of time delays between signals, rating the general dependence of one signal on another, or recovery of a given signal within noise. (l) In this application, it is meant to rate the relationship between two profile measurements, often when one of them is deemed to be correct. The method is adapted to detect a longitudinal distance offset between profiles and rate the agreement between them when the offset is removed. An extension to the method is also proposed for some applications when a linear distance measurement error exists.

The output of the cross correlation method yields much of the diagnostic information that is provided by the coherence and gain plots, but can be summarized in a single value for a given index of interest, or one value per waveband. When the method is customized for a given index, a high rating requires that the overall roughness level of two profiles is equivalent and that both of them distribute roughness equally within a profile. For example, when the method is applied to the IRI, a high rating requires that features which contribute to the IRI appear in the same locations with the same shape. This qualifies the method as a good candidate for certifying profilers for construction quality control, where the ability of a profiler to locate and prioritize isolated rough spots is important.

\section{Theoretical Development}

Cross correlation values are obtained by performing a convolution integral between two profiles with a given longitudinal offset. A cross correlation function is a collection of correlation values expressed versus longitudinal offset. If profiles were truly random functions, the correlation values would be zero at all values of offset except zero. Profiles are not random functions, and repeat measurements are never completely synchronized. Therefore, correlation functions between profiles of the same site fluctuate with distance, but are expected to reach a peak level at the offset needed to synchronize them.

For two measures of road profile, the cross-correlation function is defined as:

$$
\mathrm{R}_{\mathrm{pq}}(\delta)=\lim _{\mathrm{L} \rightarrow \infty} \frac{1}{\mathrm{~L}} \int_{0}^{\mathrm{L}} \mathrm{P}(\mathrm{x}) \mathrm{Q}(\mathrm{x}+\delta) \mathrm{dx}
$$

where $\mathrm{P}$ and $\mathrm{Q}$ are each measurements of road profile as a function of distance $\mathrm{x}$. The cross correlation function, $R$, exists as a continuous function of the offset distance $d$ between the profiles. Actual measures of road profile are finite in length. For a given length, $\mathrm{L}$, the cross correlation function can be estimated by:

$$
\mathrm{R}_{\mathrm{pq}}(\delta) \approx \frac{1}{\mathrm{~L}} \int_{0}^{\mathrm{L}} \mathrm{P}(\mathrm{x}) \mathrm{Q}(\mathrm{x}+\delta) \mathrm{dx}
$$

Since the profile is sampled at discrete intervals, the integral must be replaced by a summation: 


$$
\mathrm{R}_{\mathrm{pq}}(\delta) \approx \frac{1}{\mathrm{~N}} \sum_{\mathrm{i}=1}^{\mathrm{N}} \mathrm{P}_{\mathrm{i}} \mathrm{Q}_{\mathrm{i}+\delta / \Delta \mathrm{x}}
$$

where the subscripts indicate discrete sample numbers, collected at an interval of Dx. The number of samples, $\mathrm{N}$, is the value needed to cover the overall length of interest. (The value of $\mathrm{N}$ will be the highest integer value that does not exceed L/Dx.) Using equation 6 requires that the offset value $d$ is an integer multiple of the sample interval.

Equation 6 has two weaknesses when applied to road profiles. First, it yields a cross correlation function in units of elevation squared. A more desirable rating system would be normalized to produce a value of 1 for perfect correlation:

$$
\rho_{\mathrm{pq}}(\delta)=\frac{1}{\sigma_{\mathrm{P}} \sigma_{\mathrm{Q}}} \sum_{\mathrm{i}=1}^{\mathrm{N}} \hat{\mathrm{P}}_{\mathrm{i}} \hat{\mathrm{Q}}_{\mathrm{i}+\delta / \Delta \mathrm{x}}
$$

Where the hats over the letters "P" and "Q" indicate that the profiles are offset vertically to have a mean value of zero. The values $s_{P}$ and $s_{Q}$ represent the standard deviation of profiles $\mathrm{P}$ and $\mathrm{Q}$, respectively. Equation 7 produces a - 1 to 1 rating of the correlation, and will only produce a value of 1 when the shape of both profiles are exactly the same and they are synchronized. This is because the estimated cross correlation function is normalized by the product of the standard deviation of each profile.

A second weakness is that differences in overall roughness are not penalized by the standard cross correlation function. Two profiles that have the exact same shape but very different amplitudes would be rewarded with a perfect rating by equation 7 . To compensate for this, the following factor is applied to the normalized cross correlation function:

$$
f=\frac{\min \left(\sigma_{\mathrm{P}}, \sigma_{\mathrm{Q}}\right)}{\max \left(\sigma_{\mathrm{P}}, \sigma_{\mathrm{Q}}\right)}
$$

This adjustment factor diminishes the value of correlation when the standard deviation of the profiles are not equal.

The recommended procedure for applying this method requires the profiles to be filtered, so their mean values are expected to be small. Nevertheless, the procedure also includes removal of the mean value. The computation is most efficient when each profile is shifted vertically to have a mean of zero and normalized by its standard deviation before the cross correlation function is calculated. Unfortunately, this may lead to errors when the roughness in the longer profile, "Q", is not evenly distributed along its length. Instead, the profile must be shortened, or "cropped", to include only the $\mathrm{N}$ samples needed each time the summation is performed. This is much less efficient, because the removal of the mean, calculation of the standard deviation, and filtering must be repeated for each value of offset. $^{2}$ (In equation 7, the range of profile "P" never changes so it only needs to be conditioned once, but " $Q$ " must be conditioned every time.)

\footnotetext{
${ }^{2}$ Removal of the mean and calculation of the standard deviation can be done very efficiently. Each time the offset (d) is advanced by a value of Dx (one sample), the influence of the point that is left behind is subtracted from the previous total and the influence of the new point is added.
} 
It is essential that the same filters be applied to both profiles before using this analysis. If the profiles are not filtered similarly the results will be clouded by the differences in waveband. It is also helpful to convert the profiles from elevation to slope before computing the correlation coefficient. If elevation is used, the agreement for the longest wavelength range included in the analysis has a disproportionate influence on the results. Whatever filter is used, the best practice is to filter the profile " $Q$ " each time the summation is performed. This is required to ensure that the effect of the filter initialization is the same in both profiles. The drawback is the filter must be applied every time a point in the cross correlation function is generated. This can be avoided if the filter initialization is not considered important by measuring a significant amount of profile ahead of the segment of interest. When that is done, both profiles may be filtered just once, as long as no part of either profile that is affected by the initialization falls within the range of samples called for by equation 7 .

\section{Synchronization}

For research studies that involve several measurements of the same road section by a single device or a collection of devices, it is often desirable to "synchronize" the profiles by adjusting their longitudinal offset to make sure they all cover exactly the same stretch of road. A common way to synchronize a set of profile measurements is to simply plot them and read a distance offset from the plots. Since cross correlation provides a rating of agreement between profiles as a function of offset, it can be used to automate this process. The procedure is based on matching two measurements of a section of road and finding the offset associated with the highest level of correlation. If the profile measurements are filtered and normalized as described above, the output of the algorithm is a number between -1 and 1 that describes the agreement of the two measurements at each offset.

Figure 11 shows a cross correlogram, which displays the cross-correlation between a measurement by a lightweight inertial profiler and a slow-speed reference measurement as a function of offset. Both were converted to slope profile and band-pass filtered to include only content in the wavelength range from about 1.5 to 7.6 meters. Because this road profile is very similar to a random signal, the level of correlation is very poor except where the measurements are synchronized. The function has a value less than 0.2 everywhere except when the longitudinal distance offset is near 0.3 meters. The peak value of 0.898 occurs at the correct offset of 0.35 meters.

In the 1993 Road Profiler Users' Group (RPUG) calibration studies, an artificial bump was placed before and after each road section to help identify the segment of interest. (9) A simple bump finder was used to synchronize the sections. The profiles were then synchronized a second time using cross correlation to verify its use for this purpose. (10) This is described in Appendix A.

This procedure works extremely well for road profiles that are random in quality. Profiles with significant periodic content, on the other hand, may show peaks at several locations. A profile of heavily curled concrete, for example, may show a sharp increase in correlation at a regular interval equal to the slab length. Figure 12 shows and example. The figure shows a cross correlogram from two measurements of Arizona site 0213 from the Long-Tern Pavement Performance (LTPP) Study. They synchronize at an offset of 0.35 
meters where the correlation level is 0.938 (which also indicates excellent agreement). On pavements with periodic content, a peak in correlation is not enough to indicate the proper offset. In most cases a peak in correlation that is much higher than the others will appear at the proper offset. When this is not true, a second cross correlogram should be calculated that includes long wavelengths only, and excludes periodic effects by ignoring the range affected by slab behavior.

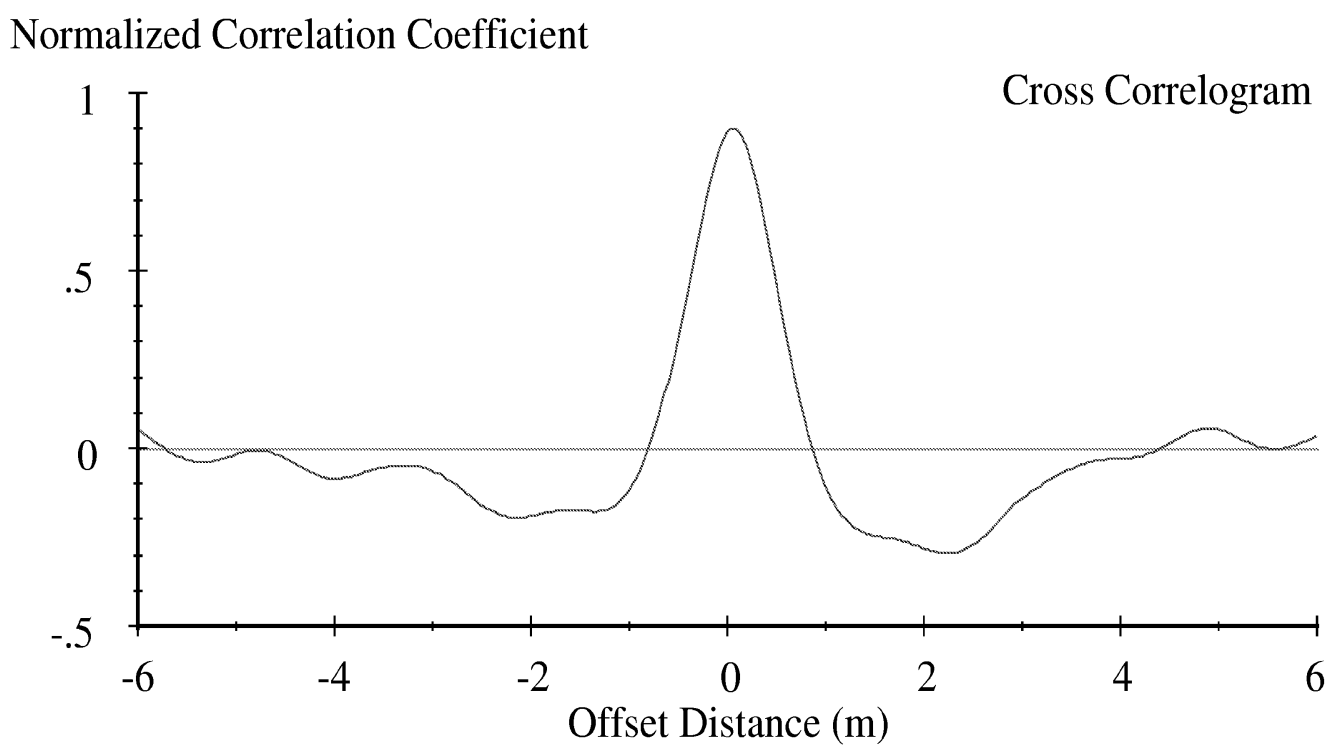

Figure 11. Cross correlogram of two profiles.

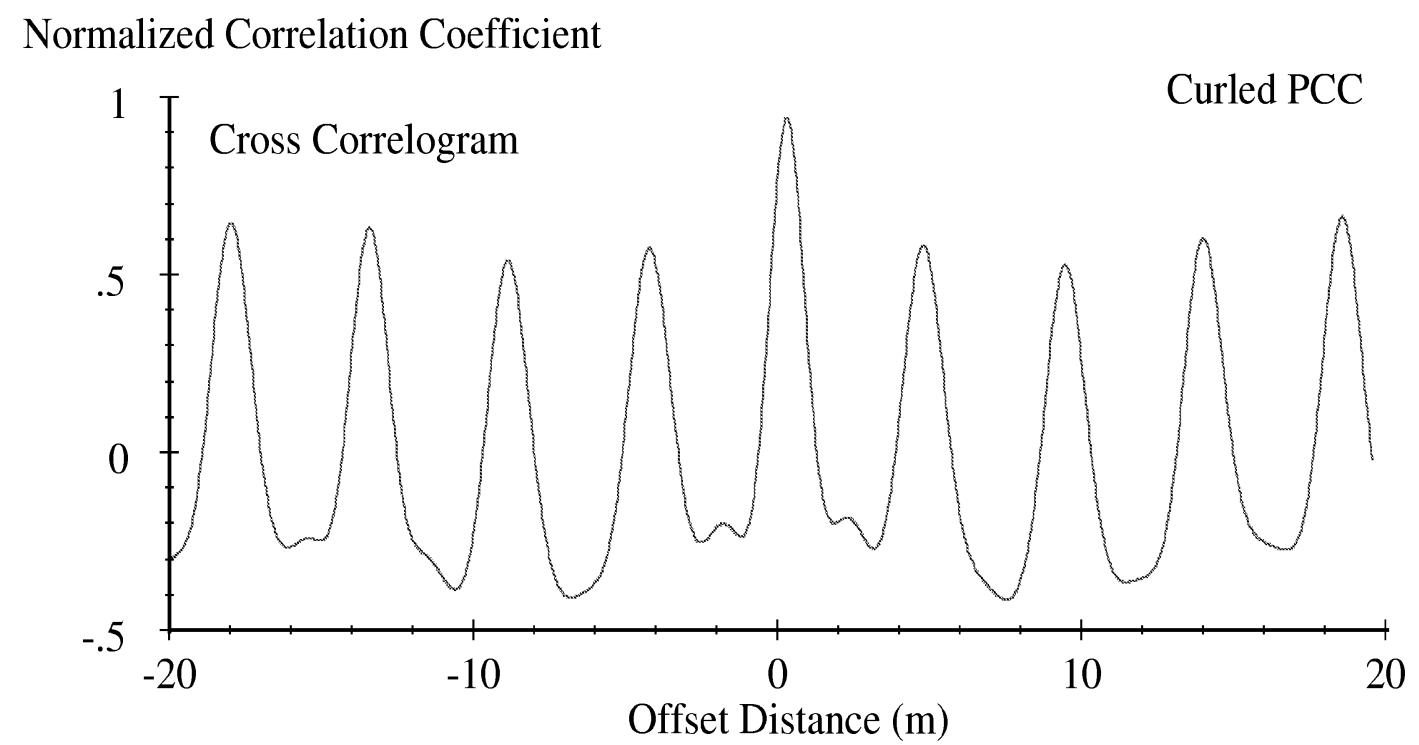

Figure 12. Cross correlogram of two profiles with periodic content.

\section{Rating of Agreement}

If the measurements compared in figure 11 agreed perfectly, the maximum correlation coefficient would be unity. However, differences between the measurements, even when they are lined up properly, still exist. This lowers the maximum correlation level. Once two measurements are synchronized, the peak correlation value used to establish their 
longitudinal offset provides a quantitative rating of the agreement between the them. This can be used to rate the agreement between two measurements from the same instrument (repeatability), measurements from unlike instruments (reproducibility), or agreement of a measurement to a reference profile (accuracy). Regardless of the type of comparison, cross correlation yields a rating of agreement in the waveband of interest when the profiles have been filtered identically.

Using cross correlation to evaluate agreement between profile measurements is much more rigorous than comparison of summary roughness indices. Two profilers might produce the same index value even though the profiles are not the same. In contrast, cross correlation of filtered profiles requires the same level of roughness and that rough features appear in the same location and have the same shape in each. Thus, it does not reward compensating error. This reduces the number of repeat measurements needed to reveal profile measurement problems. This method also offers the ability to diagnose measurement errors by considering a variety of wavebands. For example, bad agreement for short wavelengths but good agreement for long wavelengths suggests a problem with the height sensors and the opposite often suggests a problem with the accelerometer.

A powerful adaptation of this method is to pass two profiles through the IRI algorithm, then cross-correlate the filtered output. This has the advantage of comparing only those aspects of the profile that are important to the IRI and applying appropriate weighting to them. (Of course, if another index is of interest, filter the profile using its algorithm.) High correlation using this procedure requires not only that the overall IRI values match, but that the roughness is spatially distributed the same way in both measurements. This may be important if the profiles are intended for location of isolated rough spots, or if they are to be used in construction quality control.

Figure 13 provides an example of profiles with very high correlation. The figure shows three repeat measurements by a device after they have passed through the filters in the IRI algorithm. These signals compare to each other with an average correlation higher then 0.995. Note that the traces overlay so well that they are barely distinguishable from each other. Figure 14 provides an example of moderate correlation. It shows three repeat measurements from the same device on a different pavement section after they have passed through the filters in the IRI algorithm. These compare with an average correlation of about 0.84 . The traces do not overlay nearly as well, and do not agree on the severity of roughness in locations of elevated IRI. At few locations, such as 74 and 76 meters, concentrated roughness appears in only one or two of the measurements. 


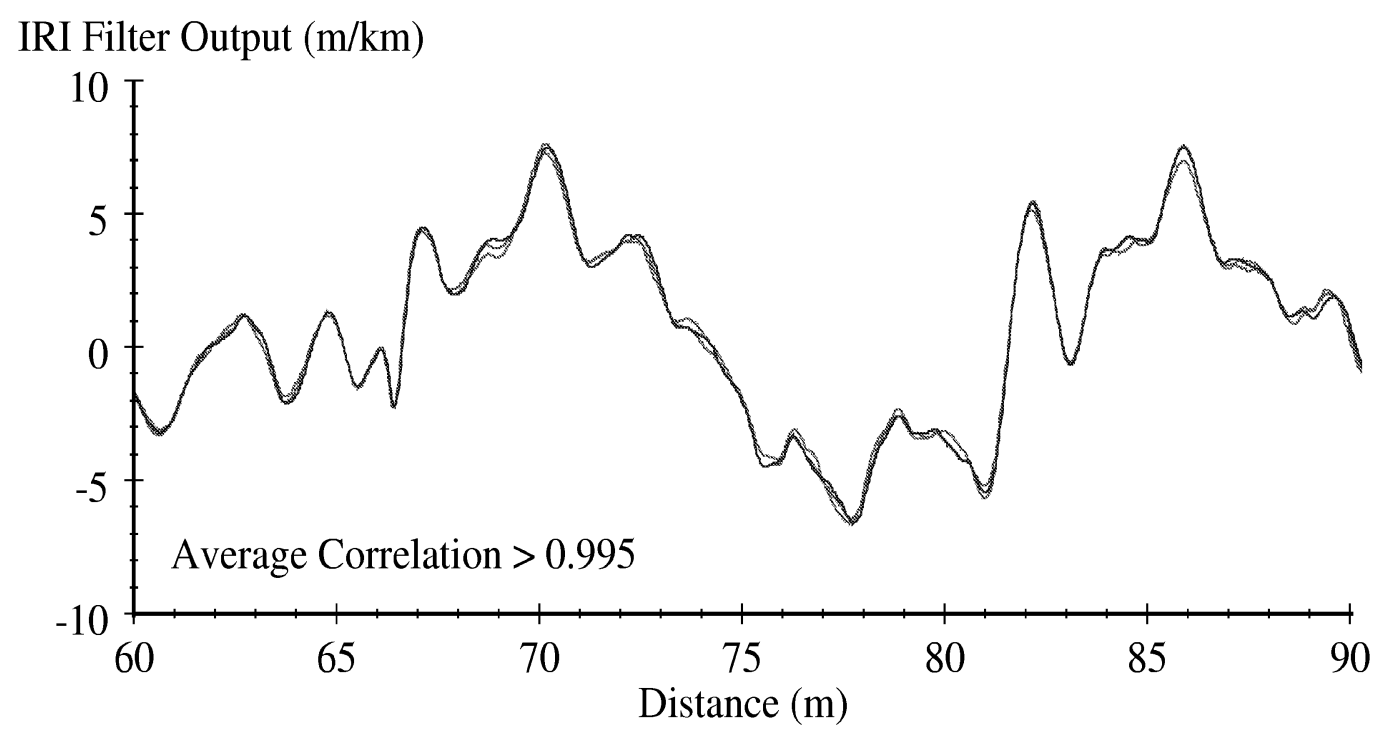

Figure 13. Three highly correlated repeat measurements.

IRI Filter Output $(\mathrm{m} / \mathrm{km})$

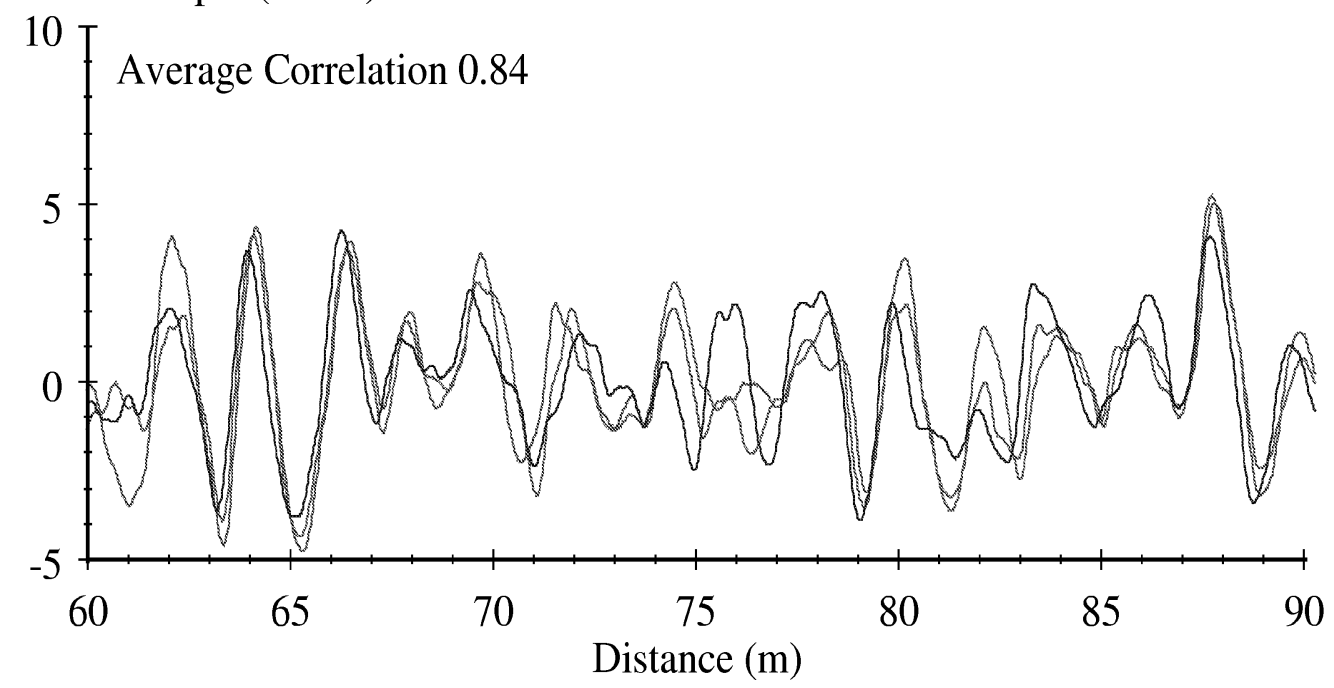

Figure 14. Three moderately correlated repeat measurements.

\section{Processing STEPS}

\section{Synchronization}

Synchronization of profiles using cross correlation is performed with the following steps:

Step 1: Identify a fixed profile that is already consistent with the desired longitudinal reference. It will be considered the location reference. The profile will have a sample interval Dx, a total length $\mathrm{L}_{\mathrm{Q}}$, and a total number if samples $\mathrm{N}_{\mathrm{Q}}$ $\left(=\mathrm{L}_{\mathrm{Q}} / \mathrm{Dx}\right)$.

Step 2: Cut a segment out of the correlated, or shifted, profile of shorter length than the reference profile, $\mathrm{L}_{\mathrm{p}}$. Preprocess it as follows. 
Step 2a: Filter it.

Step 2b: Interpolate the filtered profile to the sample interval of the reference profile. The result is the profile $\mathrm{q}$, which is a portion of the original profile $\mathrm{P}$. It will have $\mathrm{N}_{\mathrm{p}}$ samples $\left(=\mathrm{L}_{\mathrm{p}} / \mathrm{Dx}\right)$.

Step 2c: Offset the profile vertically so that the mean is zero.

Step 2d: Calculate the variance of the filtered, interpolated, and shifted profile $\hat{\mathrm{p}}$. The Result is $\mathrm{s}_{\mathrm{q}}$.

Step 3: Apply a negative offset $\left(\mathrm{d}_{0}\right)$ to the correlated profile so that the first point in it is also the first point in the reference profile. This value of offset is equal to $\mathrm{X}_{\mathrm{sQ}}-\mathrm{X}_{\mathrm{sp}}$, where $\mathrm{X}_{\mathrm{SQ}}$ is the longitudinal position of the start of the broader reference profile $(\mathrm{Q})$, and $\mathrm{X}_{\mathrm{sp}}$ is the longitudinal position of the start of the correlated profile.

Step 4: Extract the portion of the reference profile that is covered by the correlated profile. The extracted segment will cover $\mathrm{N}_{\mathrm{p}}$ samples.

Step 4a: Filter it.

Step 4b: Offset the result vertically so that the mean is zero. The result is $\hat{\mathrm{q}}$. Note that this signal must be conditioned after it has been extracted from the broader reference profile. This ensures equal application of end conditions in the two signals that will be correlated in equation 9.

Step 4c: Calculate the variance of the filtered and shifted profile over the range of interest $\left(\mathrm{s}_{\mathrm{q}}\right)$.

Step 5: Cross-correlate the signals. In this application, the variance must be calculated over the segment of interest only to account for the common situation in which the broader profile is not stationary.

$$
\begin{gathered}
\rho_{\mathrm{m}}=\frac{1}{\sigma_{\mathrm{p}} \sigma_{\mathrm{q}}} \sum_{\mathrm{i}=1}^{\mathrm{N}_{\mathrm{p}}} \hat{\mathrm{p}}_{\mathrm{i}} \hat{\mathrm{q}}_{\mathrm{i}+\mathrm{m}} \\
\delta_{\mathrm{m}}=\mathrm{X}_{\mathrm{sQ}}-\mathrm{X}_{\mathrm{sp}}+\mathrm{m} \cdot \Delta \mathrm{x}
\end{gathered}
$$

The equation 9, the value " $m$ " is the number of samples that are skipped at the start of profile $Q$. In the first application, $m$ is zero, and the offset between profiles is $X_{\mathrm{sQ}}-\mathrm{X}_{\mathrm{sp}}$. (The value of $\mathrm{m}$ is incremented from 0 to $\mathrm{N}_{\mathrm{Q}}-\mathrm{N}_{\mathrm{p}}$.)

Step 6: Shift the offset of the correlated signal by a distance equal to the sample interval of the reference profile. This amounts to shifting ahead one sample on the reference profile. (Each time this is done, increment the value of " $\mathrm{m}$ ".)

Step 7: If the end of the reference profile has not been reached, return to step 4.

The offset that corresponds to the highest value of $r$ is the proper offset for synchronization. Note that the choice of a reference profile in this process does not 
necessarily mean that it is correct. Often, this process is simply a way to make the location referencing consistent between measurements.

Step $2 \mathrm{a}$ and $4 \mathrm{a}$ require that the profiles are filtered. It is essential that both filters are equivalent. Further, the filters must remove enough of the waveband of each profile to eliminate differences in the filtering used to produce the original measurements. In other words, when the profiles are entered into equation 9 their expected wavelength content must be equal. If the filters are chosen carefully, they will obscure differences in long and short wavelength cutoff and filter shape used by the device. For example, if the profiles are to be used strictly for the calculation of IRI, the synchronization should be done using the output of the filters from the IRI algorithm. These filters produce a slope profile that covers a wavelength range from about 1.2 to 30 meters. This is well within the intended valid waveband of most profilers. Several other filtering options are possible. The IRI filter, and five other alternatives, are listed in the following section.

The interpolation of the "correlated" profile in step $2 \mathrm{~b}$ must be performed with care. If the sample interval of the correlated profile is similar to the sample interval of the reference profile or much larger, apply direct linear interpolation. However, if the sample interval of the reference profile is much larger than that of the correlated profile, direct interpolation is not sufficient. Conditioning must be applied to the correlated profile that is equivalent to that of the reference profile. This could include direct application of an anti-aliasing filter. It may also require modeling of the physical attributes of a low-speed reference device. For example, the application of a tire bridging or enveloping filter that reproduces the manner in which the reference device contacts the pavement.

The process outlined above provides a rating of agreement between profiles as a function of offset distance. Often, measurements differ in their distance measurement accuracy as well as their longitudinal referencing. Even small errors in measurement of longitudinal distance may compromise the correlation level. This occurs when the ratio of the smallest wavelength of interest to the overall length of the profile is on the same order of magnitude as the longitudinal distance measurement error level.

Cross correlation can also be used to quantify linear distance measurement error. This requires that correlation level is expressed as a function of both offset distance and distance measurement error level. The combination of offset distance and sample interval correction factor that produce the highest correlation to the reference are then considered "correct".

\section{Rating of Agreement}

The same process described above can be used for rating of agreement between profiles. Repeatability is rated by comparing two measurements by the same profiler on the same segment of road. When this is done, it does not matter which of the measurements is considered the "reference". This is because the sample intervals will be equal, and the process has reciprocity. (That is, the same result is obtained if the reference and correlated profiles are switched.) When profiles of unlike sample interval are compared, as will usually be the case for ratings agreement between two profilers, the choice of which measurement is considered the reference can be important. This is because the "candidate" profile measurement will be interpolated to have the same sample interval as the chosen 
reference. In addition, the method used to measure a road datum plane (i.e., the height sensor footprint) is deemed correct in the reference measurement.

The method of cross correlation described above for synchronization can be used directly, with the exception that the output is the correlation level $\left(r_{m}\right)$. Typically, rating of agreement and synchronization are performed concurrently with this method. It is important to allow for a modest range of longitudinal offset between two profiles under comparison, even when synchronization is already done by some other method. This is because profiles may exhibit optimal synchronization at slightly different offsets when different wavebands are considered. This depends on the phase shift of the filtering done by each profiler.

When rating agreement between profiles, the output of the cross correlation method is the correlation level at the optimum longitudinal offset (and sample interval correction, if it was included). This can be calculated using equation 9, but the scale factor of equation 8 must be applied to the value of $r_{m}$ :

$$
\text { Agreement Level }=\rho_{\mathrm{m}} \cdot f
$$

This penalizes the correlation level by the ratio of the variance of each signal, so two profilers must have the same level of roughness, in addition to the same shape. (It is equivalent to requiring a line of equality, instead of a best-fit line.)

Five filtering options for rating agreement are recommended :

1. The output of the IRI algorithm. This is a slope profile with frequency weighting determined by the quarter car filter using the Golden Car parameters.

2. The output of the RN algorithm. This is a slope profile with frequency weighting optimized to predict user panel ratings from experiments in Ohio and Minnesota.

3. The slope profile, passed through a four-pole Butterworth filter with cutoff wavelengths of 8 and 40 meters.

4. The slope profile, passed through a four-pole Butterworth filter with cutoff wavelengths of 1.6 and 8 meters.

5. The slope profile, passed through a four-pole Butterworth filter with cutoff wavelengths of 0.32 and 1.6 meters.

The first two options are meant to emphasize content in the profiles that is relevant to the accumulation of each index. The other three filters were included to help diagnose the source of disagreement between profiles by isolating each waveband. All of these filters are described in detail elsewhere. (4) Conversion to slope is a prominent feature of all five recommended filtering options. This is because most profiles exhibit much less variation in slope amplitude than elevation amplitude over the wavelength range of interest. Thus, using slope prevents the long wavelength portion of the filtered profile from dominating the results. 


\section{DEMONSTRATION USING 1993 RPUG DATA}

Data from the 1993 RPUG study were used to establish a relationship between cross correlation level and expected error in overall IRI measurement. Since measurements of IRI may agree between two measurements because of compensating error, a variety of IRI error levels are possible at the same level of cross correlation between profiles. It was therefore rather difficult to establish this relationship. All profile measurements of a given wheeltrack from the 1993 RPUG experiment were compared to each other by cross correlating their filtered IRI output and by calculating the percent difference between their overall IRI values. When every possible pair of profilers were examined this way, 374,758 pairs of cross correlation and percent IRI error were generated. The 95th percentile error level in IRI at each cross correlation level was then obtained. This involved a tremendous amount of simple analysis and data assembly, and is described in detail in Appendix A, B, and $\mathrm{C}$.

The results of these analyses are summarized in figure 15. The figure shows the average, RMS, and 95th percentile error in IRI observed at each cross correlation level above 0.85 . A clear relationship exists between cross correlation of IRI filter output and the 95th percentile level of error in IRI that may be expected on a site of similar roughness and surface texture.

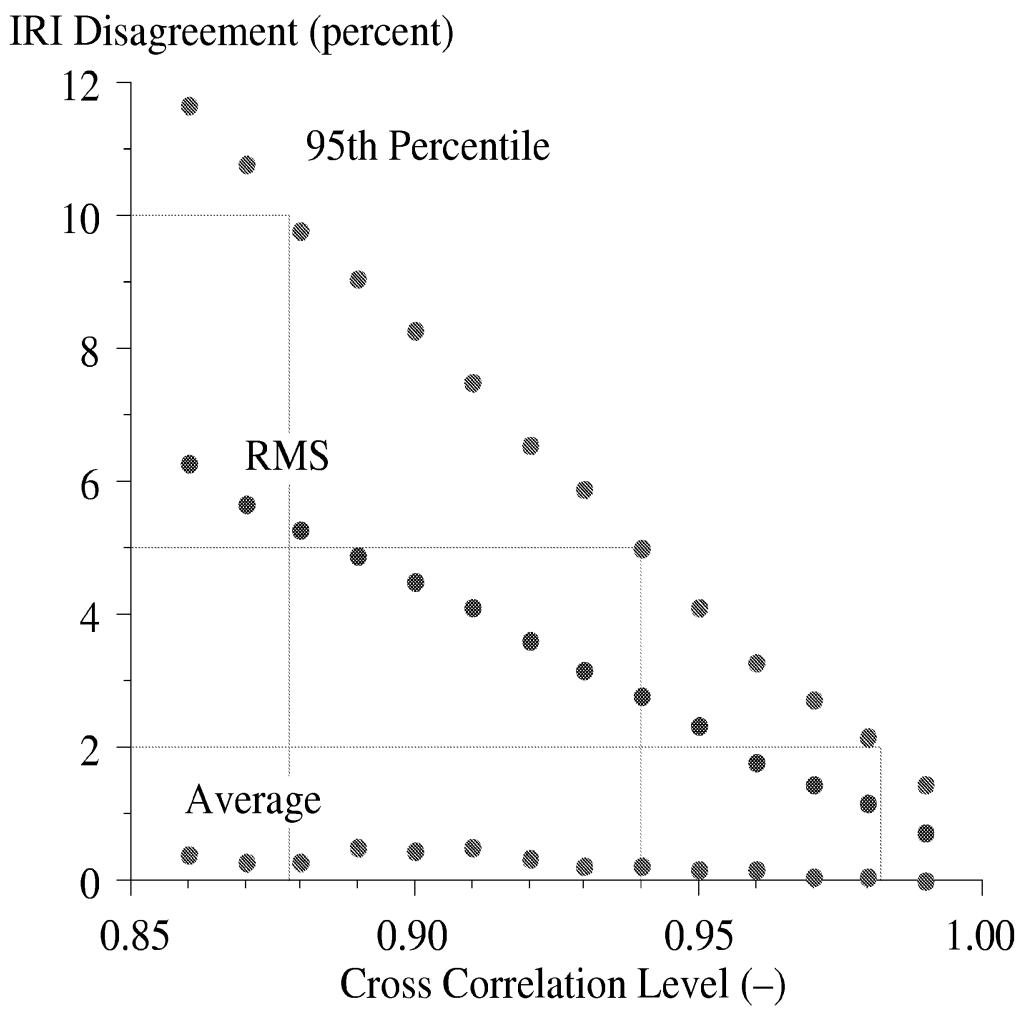

Figure 15. IRI agreement associated with cross correlation level.

\section{RECOMMENDATIONS}

It is proposed that cross correlation of filtered profile replace the precision and bias criteria of ASTM E-950 as the standard method of rating profile agreement. Cross 
correlation has the potential to provide a rating of agreement between profiles in a given waveband, and may be used successfully over a broader band of wavelengths if the filtering is done properly. In particular, overall profile agreement should be judged using filtered profile slope, and expected agreement in overall IRI and spatial distribution of IRI can be judged by comparing IRI filter output. Since the method is indifferent to which type of profiler makes each measurement when two profiles are compared, threshold limits on cross correlation level may be set that pertain to repeatability as well as accuracy.

Data from the 1993 RPUG were used to establish a relationship between cross correlation level of IRI filter output and agreement in overall IRI over 161 meters of road. The following thresholds are proposed for various "classes" of profiler:

- Research Class: At a cross correlation level of 0.98, you may expect your overall IRI measurements to agree within 2 percent of each other 95 percent of the time.

- Project Class: At a cross correlation level of 0.94, you may expect your overall IRI measurements to agree within 5 percent of each other 95 percent of the time.

- Network Class: At a cross correlation level of 0.88, you may expect your overall IRI measurements to agree within 10 percent of each other 95 percent of the time.

Further, a value of 0.94 could also be used established as a threshold for construction quality control. This must be verified by testing several profilers on new or very smooth pavement with the appropriate surface texture of each pavement type to establish that the limit is correct. In addition, the analyses must set an independent threshold that will guarantee repeatable and accurate output of the prevailing method of locating must-grinds or isolated rough spots.

Please note that the threshold correlation values were set somewhat conservatively. The data shown in figure 15 are the basis for the recommended threshold values. In figure 15, a given value of cross correlation actually represents the upper limit of a range that is 0.01 units wide. The "Project Class" threshold of 0.94, therefore, is established because values from 0.93 to 0.94 exhibited the desired performance.

The cross correlation method must be applied very carefully. Analysis of the 1993 RPUG data, and other recent studies (11), show that profilers will exhibit a different level of repeatability and accuracy on different types of pavement. Achieving one of the class levels listed above on a given pavement type only implies that good performance is expected on pavement of the same type and level of roughness, and of the same surface texture. Therefore, selection of test sites for profiler verification and classification is very important.

Profiling technology has improved significantly since the 1993 RPUG study. It is highly recommended that data from a more recent experiment are used to verify the threshold levels for overall performance, and develop threshold levels for verifying a profiler's use in locating candidate hot spots for corrective action. Data are also needed to ensure that the thresholds maintain their relevance for other segment lengths. (The expected IRI error levels will change, but the trends must be verified.) In the best case, a new experiment would be designed with the help of an experienced statistician so that the threshold values above could be verified with a sufficient, but minimal experiment. 
This study included theoretical derivation of the proper segment length, longitudinal distance error tolerance, phase shift limits, and sampling rules needed to apply the method. Combinations of white noise slope, elevation, and acceleration were used to generate artificial road profiles using common rules from the literature. (12) The cross correlation method was then applied to these profiles in an attempt to study the issues listed above. (Note that many of the results in this type of study can be derived directly in closed form, so this type of exercise is often a waste of time.) The results of these efforts are not documented here, because they were found to disagree with practical observations made in the field, and the results of a recent experiment. (11) The main weakness of this approach, especially in the study of sampling issues, is the lack of realistic spectral content in the short wavelength and macrotexture range. It is recommended that the artificial profiles be replaced by detailed profile measurements by a profiler with a sample interval shorter than 0.2 inches and a height sensor footprint with a radius of 0.1 inches or smaller. These measurements would replace the artificial profiles in the study.

\section{REFERENCES}

1. Bendat, J.S. and A.G. Piersol, Random Data: Analysis and Measurement Procedures. Wiley-Interscience, New York, (1971).

2. "Standard Test Method for Measuring the Longitudinal Profile of Traveled Surfaces with an Accelerometer Established Inertial Profiling Reference." ASTM Standard E950, Annual Book of ASTM Standards, Vol. 04.03, pp. 692-696.

3. Robson, J. D., "Road Surface Description and Vehicle Response." International Journal of Vehicle Design, Vol. 1, No. 1 (1979) pp 25-35.

4. Sayers, M. W. and Karamihas, S. M., "Interpretation of Road Roughness Profile Data." Federal Highway Administration, FHWA/RD-96/101 (1996) 177 p.

5. Fernando, E. G., "Evaluation of Accuracy of Surface Profiles." Transportation Research Record 1699 (2000) pp. 127-133.

6. Li, Y., and Delton, J., "Approaches to Evaluation of Profiler Accuracy." Compendium of Papers, 82nd Annual Meeting of the Transportation Research Board (2003).

7. Prem, H., "Development and Evaluation of a Method for Validation of Pavement Roughness Measurements." ARRB Transport Research Ltd. Contract Report RE7135 (1998).

8. Prem, H., "Measurement of Reference Profiles for High-Speed Profiler Validation." Notes on Technology Exchange Workshop, Validation of Roughness Measurements, Vic Australia, March 4-5, 1999.

9. Perera, R.W. and S.D. Kohn, Road Profiler User Group Fifth Annual Meeting. Road Profiler Data Analysis and Correlation. Soil and Materials Engineers, Inc. Research Report No. 92-30, (1994) 87 p. 
10. Karamihas, S. M., et. al., "Guidelines for Longitudinal Pavement Profile Measurement. Appendix C." National Cooperative Highway Research Program Report 454 (1999).

11. Karamihas, S. M. and Gillespie, T. D., "Assessment of Profiler Performance for Construction Quality Control: Phase I." Report to the ACPA, University of Michigan Transportation Research Institute Report UMTRI-2003-03 (2002) 43 p.

12. Sayers, M.W., "Characteristic Power Spectral Density Functions for Vertical and Roll Components of road Roughness." American Society of Mechanical Engineers. AMD-Vol. 80. (1986) pp. 113-129. 


\section{Appendix A: Analysis of 1993 RPUG Data}

This appendix presents analyses that were performed on data from the 1993 RPUG experiment. In the 1993 RPUG experiment several profilers that normally operate in North America made multiple measurements of a small set of test sections. The experiment covered devices operated by state departments of transportation, LTPP regional contractors, profiler manufacturers, and other private operators. In each of four regions, profilers measured up to eight sections as many as ten times each. Although the state of profiling practice has improved since 1993, these data remain a rare source of measurements from multiple profilers on the same sections.

These data were used in a recent research study for the National Cooperative Highway Research Program (NCHRP) to examine the capabilities of common profilers in use in North America. (1) Usually, the results were used as a foundation for discussion of new experiments. The 1993 RPUG experiment served as the primary source of data in cases where a new experiment was either not warranted or not practical. The factors investigated using these measurements were (1) the accuracy of longitudinal distance measurement, (2) the repeatability of profilers from the experiment, (3) the agreement of profilers with a reference measurement, (4) the level of bias caused by coarse surface texture, and (5) the effect of operating speed on repeatability.

This appendix provides the information produced for the NCHRP study. These results serve as the basis for establishing a connection between profile cross correlation and error in IRI measurement, as described in Appendix C. A more detailed description of the 1993 RPUG experiment and the results was distributed after the 1993 RPUG meeting. (2)

\section{THE RPUG EXPERIMENT}

The 1993 RPUG experiment took place in four regions in the U.S. In each region, a state DOT prepared up to eight test sections 160.9 meters long. These sections, described in table A-1, were selected to cover range of surface type, roughness, and surface texture. Each profiler that participated in the experiment measured the sections in the region in which it operates. In most cases, the profilers measured each section ten times. Usually, five measurements were made at a speed near $80 \mathrm{kph}$ and five were made at a speed near 64 $\mathrm{kph}$. The sections were also measured using a Dipstick to provide a reference roughness value. Overall, 34 profilers took part in the study and more than 2400 measurements were made. Table A-2 lists the profilers covered in this appendix. The table provides an instrument number for each profiler that is used to identify it throughout this appendix. The table also lists the sensor type, sample interval, and manufacturer. If South Dakota is listed as the manufacturer, it means that the profiler was built in-house by a state highway using the concept documented by Dave Huft. (3) Many of the commercial profilers are also of South Dakota type. 
Table A-1. Sections measured in the 1993 RPUG experiment.

\begin{tabular}{|c|c|c|c|}
\hline Region & $\begin{array}{l}\text { Section } \\
\text { Number }\end{array}$ & Pavement Type & $\begin{array}{c}\text { IRI Left } \\
\text { Wheeltrack }(\mathrm{m} / \mathrm{km})\end{array}$ \\
\hline \multirow[t]{8}{*}{ Mississippi } & 1 & Asphalt Concrete & 1.25 \\
\hline & 2 & Composite & 0.89 \\
\hline & 3 & Composite & 2.55 \\
\hline & 4 & Asphalt Concrete & 3.41 \\
\hline & 5 & Portland Cement Concrete & 2.94 \\
\hline & 6 & Portland Cement Concrete & 1.28 \\
\hline & 7 & Portland Cement Concrete & 2.72 \\
\hline & 8 & Portland Cement Concrete & 1.69 \\
\hline \multirow[t]{6}{*}{ Nevada } & 1 & Asphalt Concrete & 2.79 \\
\hline & 2 & Asphalt Concrete & 0.80 \\
\hline & 3 & Asphalt Concrete & 3.55 \\
\hline & 4 & Portland Cement Concrete & 1.78 \\
\hline & 5 & Portland Cement Concrete & 1.69 \\
\hline & 6 & Portland Cement Concrete & 1.10 \\
\hline \multirow[t]{8}{*}{ Pennsylvania } & 1 & Asphalt Concrete & 2.61 \\
\hline & 2 & Asphalt Concrete & 1.06 \\
\hline & 3 & Asphalt Concrete & 2.13 \\
\hline & 4 & Asphalt Concrete & 2.36 \\
\hline & 5 & Portland Cement Concrete & 2.78 \\
\hline & 6 & Portland Cement Concrete & 3.23 \\
\hline & 7 & Portland Cement Concrete & 1.89 \\
\hline & 8 & Portland Cement Concrete & 1.40 \\
\hline \multirow[t]{8}{*}{ South Dakota } & 1 & Asphalt Concrete & 3.89 \\
\hline & 2 & Asphalt Concrete & 1.30 \\
\hline & 3 & Asphalt Concrete & 1.42 \\
\hline & 4 & Asphalt Concrete & 1.09 \\
\hline & 5 & Portland Cement Concrete & 1.54 \\
\hline & 6 & Portland Cement Concrete & 1.37 \\
\hline & 7 & Portland Cement Concrete & 1.73 \\
\hline & 8 & Portland Cement Concrete & 1.45 \\
\hline
\end{tabular}


Table A-2. Devices that participated in the 1993 RPUG experiment.

\begin{tabular}{|c|c|c|c|c|c|c|c|}
\hline Region & Inst. \# & Make & Model & $\begin{array}{l}\text { Sensor } \\
\text { Type }\end{array}$ & $\begin{array}{c}\text { Sample } \\
\text { Interval } \\
(\mathrm{mm})\end{array}$ & Tracks & $\begin{array}{c}\text { Number of } \\
\text { Measurements }\end{array}$ \\
\hline \multirow[t]{6}{*}{ M } & MDS & Dipstick & & I & 305 & B & 8 \\
\hline & M01 & ICC & MDR 4090 & $\mathrm{U}$ & 327 & $\mathrm{~L}$ & 80 \\
\hline & M02 & ICC & MDR 4087 & $\mathrm{U}$ & 332 & B & 79 \\
\hline & M03 & ICC & MDR $4087 \mathrm{~L}$ & $\mathrm{~L}$ & 165 & B & 58 \\
\hline & M05 & Pave Tech & & $\mathrm{U}$ & 263 & B & 80 \\
\hline & M06 & K.J. Law & $6900 \mathrm{DNC}$ & $\mathrm{O}$ & 152 & $\mathrm{~B}$ & 100 \\
\hline \multirow[t]{6}{*}{$\overline{\mathrm{N}}$} & $\mathrm{N03}$ & ICC & MDR 4090 & $\mathrm{U}$ & 326 & $\mathrm{~B}$ & 59 \\
\hline & N04 & ICC & MDR 4097 & $\mathrm{U}$ & 328 & $\mathrm{~B}$ & 59 \\
\hline & N06 & ICC & & $\mathrm{U}$ & 302 & B & 60 \\
\hline & N07 & ICC & MDR $4090 \mathrm{~L}$ & $\mathrm{~L}$ & 160 & B & 61 \\
\hline & N08 & K.J. Law & $690 \mathrm{DNC}$ & $\mathrm{O}$ & 152 & B & 60 \\
\hline & N09 & K.J. Law & $6900 \mathrm{DNC}$ & $\mathrm{O}$ & 152 & $\mathrm{~B}$ & 58 \\
\hline \multirow[t]{13}{*}{$\mathrm{P}$} & PDS & Dipstick & & I & 305 & B & 8 \\
\hline & P01 & ProRut & & $\mathrm{L}$ & 50 & B & 80 \\
\hline & P02 & $\mathrm{ICC}$ & MDR 4090 & $\mathrm{U}$ & 333 & B & 80 \\
\hline & $\mathrm{P} 03$ & ICC & & $\mathrm{U}$ & 331 & $\mathrm{~B}$ & 80 \\
\hline & P04 & ICC & 4900 LaserSDP & $\mathrm{L}$ & 101 & $\mathrm{~B}$ & 80 \\
\hline & P05 & K.J. Law & $690 \mathrm{DNC}$ & $\mathrm{O}$ & 152 & B & 80 \\
\hline & P06 & ICC & MDR 4097 & $\mathrm{U}$ & 319 & $\mathrm{~B}$ & 78 \\
\hline & $\mathrm{P} 07$ & K.J. Law & $6900 \mathrm{DNC}$ & $\mathrm{O}$ & 152 & $\mathrm{~B}$ & 80 \\
\hline & P08 & ICC & MDR $4087 \mathrm{~L}$ & $\mathrm{~L}$ & 165 & B & 74 \\
\hline & P73 & ICC & MDR 4195 & $\mathrm{U}$ & 342 & B & 80 \\
\hline & P74 & ICC & MDR 4195 & $\mathrm{U}$ & 342 & $\mathrm{~B}$ & 79 \\
\hline & P75 & ICC & MDR 4195 & $\mathrm{U}$ & 343 & B & 80 \\
\hline & P76 & ICC & MDR 4195 & $\mathrm{U}$ & 340 & $\mathrm{~B}$ & 80 \\
\hline \multirow[t]{11}{*}{$\mathrm{S}$} & SDS & Dipstick & & I & 305 & $\mathrm{~B}$ & 8 \\
\hline & S01 & ICC & & $\mathrm{U}$ & 323 & $\mathrm{~B}$ & 65 \\
\hline & S02 & South Dakota & & $\mathrm{U}$ & 305 & $\mathrm{~L}$ & 80 \\
\hline & $\mathrm{S} 03$ & Pave Tech & & $\mathrm{U}$ & 331 & $\mathrm{~L}$ & 80 \\
\hline & S04 & South Dakota & & $\mathrm{U}$ & 305 & $\mathrm{~L}$ & 77 \\
\hline & S05 & K.J. Law & & $\mathrm{O}$ & 152 & B & 47 \\
\hline & S06 & South Dakota & & $\mathrm{U}$ & 305 & $\mathrm{~L}$ & 79 \\
\hline & S08 & K.J. Law & $6900 \mathrm{DNC}$ & $\mathrm{O}$ & 152 & B & 77 \\
\hline & S10 & ARAN & 4300 LaserSDP & $\mathrm{L}$ & 204 & $\mathrm{~B}$ & 58 \\
\hline & S11 & Pave Tech & & $\mathrm{U}$ & 335 & $\mathrm{~B}$ & 40 \\
\hline & S12 & South Dakota & & $\mathrm{U}$ & 305 & $\mathrm{~L}$ & 80 \\
\hline \multicolumn{2}{|c|}{$\begin{array}{l}\text { M - Mississippi } \\
\text { I - Inclinometer } \\
\text { L - Left }\end{array}$} & \multicolumn{2}{|c|}{$\begin{array}{l}\text { - Nevada } \\
\text { O - Optical } \\
\text { B - Both (Left and Right) }\end{array}$} & $\begin{array}{l}\text { Pennsyl } \\
\text { Ultrasor }\end{array}$ & & \multicolumn{2}{|c|}{$\begin{array}{l}\text { S - South I } \\
\text { L - Laser }\end{array}$} \\
\hline
\end{tabular}




\section{LONGITUdinal Distance Measurement}

The 1993 RPUG tests were designed to eliminate errors in longitudinal positioning of the measurements. To help maintain a consistent starting position in each measurement, an artificial bump was placed on the road before and after each section. The bumps were about 6 millimeters high, 0.46 meters long, and were located 30.5 meters upstream and 15.2 meters downstream of the section of interest. The data files used in the analyses reported in this appendix were lined up using cross-correlation rather than the bumps in the profile. The cross-correlation program shifts the "zero" location of every file to match a reference measurement, usually by the Dipstick. Since the sections were all 160.9 meters long, the artificial bumps should appear 206.7 meters apart at the longitudinal locations -30.48 and 176.17 meters. This was used to check the procedure for lining up the sections.

The distance between the bumps measured by each profiler was also used to test the accuracy of their longitudinal distance measurement. Not all of the measurements included enough profile surrounding the section of interest to contain both bumps. Some other measurements were long enough, but no bump appeared in the expected location. (These cases prompted some verification to make sure the section was lined up properly.) Naturally, the measurement of longitudinal distance was only checked on measurements where both bumps appeared clearly in the profile. Table A-3 lists the results for each profiler. The table lists the number of measurements that included both bumps, the average distance between them, and the error in percent. The table also lists the average offset level in units of length. A negative offset means the profiler underestimated the distance and a positive offset means the profiler overestimated the distance. In most cases, the offset error (or bias) in longitudinal distance measured by a profiler was consistent from run to run. Thus, the average offset error represents the value expected in a single run. The offset error is listed side-by-side with the sample interval to identify cases where the error was not much larger than the sample interval.

Table A-3 demonstrates that most profilers measure longitudinal distance fairly accurately. Twenty-one of the thirty profilers measured the distance between the bumps consistently within two profile samples. Since each bump can only be detected in the profile within one sample, these twenty-one devices are considered correct. Three of the profilers exhibited an error level greater than 1 meter, or about 0.5 percent. This is more serious: A bias in longitudinal distance measurement of 0.5 percent or more should prompt recalibration of the distance measurement instrument.

The profilers that exhibited the greatest error in longitudinal distance measurement may have slowed down significantly over the bumps to avoid damage to the vehicle, or simply because the bumps were not within the section of interest in the study. If the error was caused by extremely low-speed operation, it is of lesser concern because it does not represent typical operation of the profilers. 
Table A-3. Longitudinal distance measurement accuracy.

\begin{tabular}{|c|c|c|c|c|c|c|}
\hline Region & $\begin{array}{c}\text { Inst. } \\
\text { Number }\end{array}$ & $\begin{array}{c}\text { Number of } \\
\text { Measurements }\end{array}$ & $\begin{array}{c}\text { Average } \\
\text { Separation }(\mathrm{m})\end{array}$ & $\begin{array}{c}\text { Percent } \\
\text { Error }\end{array}$ & $\begin{array}{l}\text { Offset } \\
(\mathrm{mm})\end{array}$ & $\begin{array}{c}\text { Sample Interval } \\
(\mathrm{mm})\end{array}$ \\
\hline \multirow[t]{5}{*}{$M$} & M01 & 73 & 206.15 & -0.25 & -509 & 327 \\
\hline & M02 & 76 & 208.35 & 0.82 & 1700 & 332 \\
\hline & M03 & 50 & 206.14 & -0.25 & -518 & 165 \\
\hline & M05 & 72 & 206.40 & -0.12 & -258 & 263 \\
\hline & M06 & 99 & 207.19 & 0.26 & 539 & 152 \\
\hline \multirow[t]{3}{*}{$\mathrm{N}$} & N04 & 59 & 206.51 & -0.07 & -149 & 328 \\
\hline & N08 & 60 & 206.74 & 0.04 & 88 & 152 \\
\hline & N09 & 57 & 207.75 & 0.53 & 1094 & 152 \\
\hline \multirow[t]{12}{*}{$\mathrm{P}$} & P01 & 79 & 206.41 & -0.12 & -242 & 50 \\
\hline & P02 & 80 & 206.66 & 0.00 & 8 & 333 \\
\hline & P03 & 80 & 207.34 & 0.33 & 683 & 331 \\
\hline & P04 & 76 & 206.85 & 0.09 & 193 & 101 \\
\hline & $\mathrm{P} 05$ & 70 & 206.79 & 0.07 & 137 & 152 \\
\hline & P06 & 68 & 206.61 & -0.02 & -47 & 319 \\
\hline & P07 & 80 & 206.62 & -0.02 & -34 & 152 \\
\hline & P08 & 74 & 206.24 & -0.20 & -415 & 165 \\
\hline & P73 & 79 & 206.72 & 0.03 & 62 & 342 \\
\hline & P74 & 79 & 206.59 & -0.03 & -62 & 342 \\
\hline & P75 & 80 & 206.53 & -0.06 & -128 & 343 \\
\hline & P76 & 80 & 206.69 & 0.02 & 32 & 340 \\
\hline \multirow[t]{10}{*}{$S$} & S01 & 64 & 206.18 & -0.23 & -474 & 323 \\
\hline & S02 & 78 & 206.46 & -0.09 & -191 & 305 \\
\hline & S03 & 55 & 206.46 & -0.09 & -194 & 331 \\
\hline & S04 & 65 & 206.57 & -0.04 & -80 & 305 \\
\hline & S05 & 47 & 206.51 & -0.07 & -139 & 152 \\
\hline & S06 & 70 & 205.87 & -0.38 & -786 & 305 \\
\hline & S08 & 77 & 206.48 & -0.09 & -176 & 152 \\
\hline & S10 & 53 & 203.21 & -1.67 & -3444 & 204 \\
\hline & S11 & 40 & 206.40 & -0.12 & -256 & 335 \\
\hline & S12 & 70 & 206.63 & -0.01 & -22 & 305 \\
\hline
\end{tabular}

M - Mississippi N - Nevada P - Pennsylvania $\quad$ S - South Dakota 


\section{REPEATABILITY}

The repeatability of a profiling device is its ability to produce the same result in multiple runs with minimal random error. It is very important that a profiler measure roughness with reasonable repeatability, since a device that is not repeatable has no hope of being accurate. A lack of repeatability also suggests that a random error source is present in the measurement. Some aspects of the pavement surface shape affect repeatability through no fault of a profiler. Transverse, longitudinal, and temporal variations in pavement roughness may introduce scatter into a set of measurements, even if a perfectly repeatable profiler was used.

The goal of this section is to present statistics that summarize the level of repeatability of each profiler for measurement of an overall roughness index value. The 1993 RPUG data are a good source for judging repeatability without the confounding influence of variations in pavement roughness with time and position. Each profiler visited each section once and made all of the measurements of a section in a short time span, so variations in roughness with time should not affect the results. The measurements are also lined up longitudinally. Although the lateral position of the profilers was not strictly controlled, paint marks were placed every 7.6 meters along the left wheeltrack of each section to help guide the drivers along a consistent path. At the very least, these paint marks reminded the drivers of the importance of consistent lateral positioning in the experiment.

\section{International Roughness Index}

Most of the profilers listed in table A-2 measured all of the sections in their region ten times. To quantify the scatter exhibited by a profiler, each IRI value was normalized by the average of the ten measurements on a given wheeltrack of a given section. For example, the ProRut measured eight sections ten times each. The IRI was computed for the left and right wheeltrack in each measurement, for a total of 160 roughness values. Each set of ten measurements from one side of a section was normalized by its average, and the values on all sixteen wheeltracks were compiled into a histogram, shown in figure A-1. Of course, the average of the 160 values in the figure is 1 . The scatter is an indication of the level of repeatability. The standard deviation of the values in the figure is 0.027 . This means that about 68 percent of the measurements by the ProRut were within 2.7 percent of the prevailing average for a given wheeltrack. A more relevant way to summarize the performance than the standard deviation is to set a limit for the scatter and see how many measurements fall within the limit. For example, the histogram shows that most of the roughness values (150 out of 160) measured by the ProRut are within 5 percent of the section average. If the limit is set at 2 percent, only 101 of measurements "pass."

IRI values from all of the profilers were compiled in this fashion as a means of characterizing each profiler's repeatability. The results should not be interpreted too precisely, since not every profiler made the same set of measurements. Not all of the profilers within a region measured all of the sections exactly ten times. Some profilers, usually the South Dakota type, measured only the left wheeltrack. Each region had a unique 
set of sections, so only comparisons of major trends should be made across all of the regions. Nevertheless, some of the trends are so strong that they are meaningful.

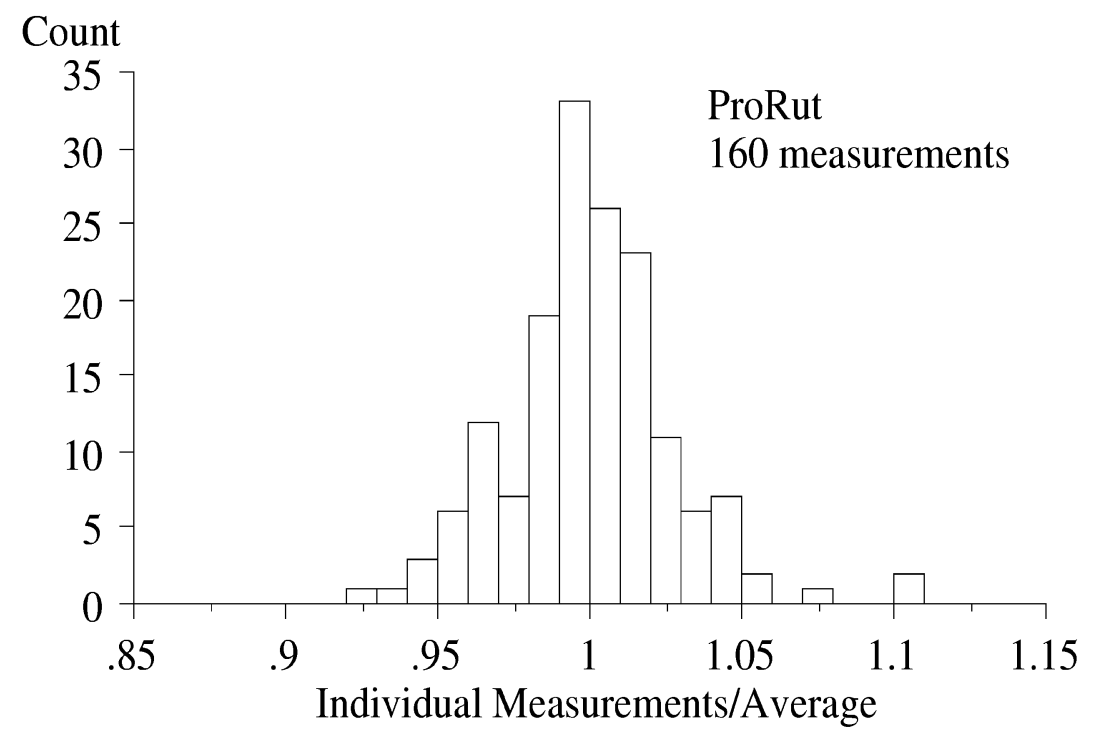

Figure A-1. Repeatability of the ProRut.

Table A-4 summarizes the results. The table provides the standard deviation, the number of measurements within 2 percent of the average, and the number of measurements within 5 percent of the average. The standard deviation is expressed as a percentage. For example, the standard deviation for the histogram in figure A-1 would be listed as 2.7 percent, rather than 0.027 .

The number of measurements within 2 percent of the average can be thought of as an indication of a profiler's ability to function as a reference device. Specific definitions aside, a profiler that claims to be "Class 1" should be able to repeat a measurement of IRI within 2 percent on a section 160.9 meters long. On the other hand, only slight deviations in lateral positioning of the measurement can cause changes in IRI larger than that. (1) Thus, a profiler may be Class 1 capable, but the combination of profiler, operator, operational procedures, and surface type may not. Of course, the performance of the overall combination of these things is a more informative measure of how a profiler is likely to work in practice. Besides, if a profiler does not include any features that aid a driver in holding a consistent lateral position, why should the resulting variations not reflect on the profiler's probable performance in the field? The same could be said for triggering, detection of bad readings, operating outside the valid speed range for the profiler, etc.

Only two of the profilers measured IRI within 2 percent of the average more than threefourths of the time. These were both K.J. Law profilers in use at the time in the LTPP study.

The number of measurements within 5 percent of the average is an indication of a profiler's sufficiency for use in network-level profiling. Meeting this requirement means that a profiler can measure a long stretch of road just once (as is usually the case in network monitoring) with confidence that IRI values of 160.9 meters long segments are probably within 5 percent of the value that the profiler would measure in several repeats. Five percent is not very restrictive, but network-level profiling does not require a high level of 
precision. Besides, the roughness of most roads varies more than 5 percent between network monitoring visits.

Table A-4. Repeatability of profilers in measurement of IRI.

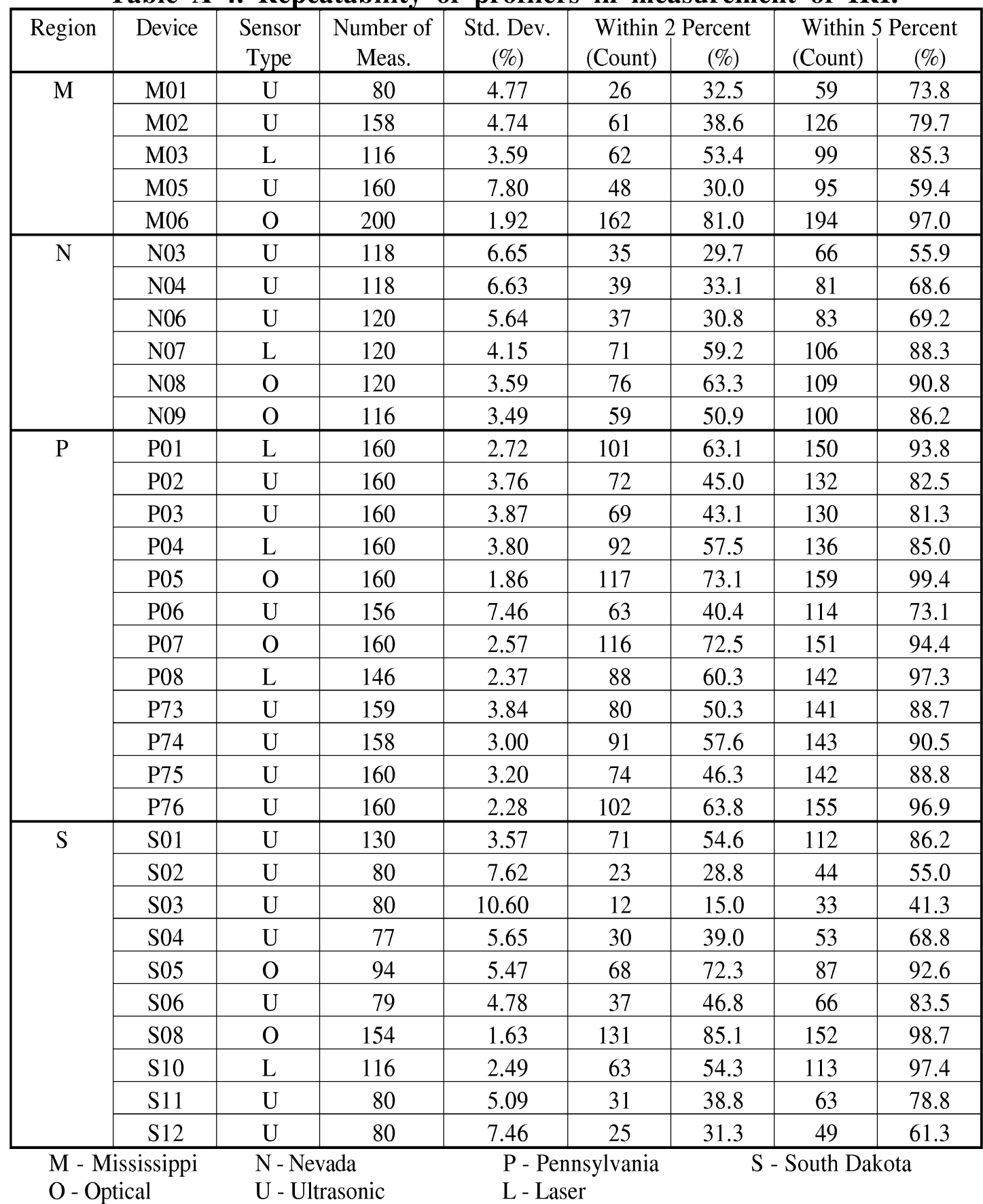

Keep in mind that the level of repeatability in percent, as expressed in this discussion, is tied very closely to the segment length. The variations in IRI would be much lower if the segment length were 1.6 kilometers, rather than 161 meters, and most of these profilers would meet the 5 percent sufficiency requirement just described.

The broad range of performance exhibited by these profilers can be attributed largely to height sensor technology. Table A-5 summarizes the performance of four broad types of profilers: (1) agency-built ultrasonic, (2) commercially built ultrasonic, (3) laser, and (4) 
optical. Strictly speaking, the normalized roughness values from different regions should not be mixed, because the differences in the test sections gives them different meaning, but they are combined anyway to illustrate the large disparity in performance between the profiler types.

Table A-5. Repeatability of profiler types in measurement of IRI.

\begin{tabular}{|l|c|c|c|c|c|c|}
\hline Profiler Type & Number of & Std. Dev. & \multicolumn{2}{|c|}{ Within 2 Percent } & \multicolumn{2}{|c|}{ Within 5 Percent } \\
& Meas. & $(\%)$ & (Count) & $(\%)$ & (Count) & $(\%)$ \\
\hline Optical & 923 & 2.95 & 670 & 72.6 & 877 & 95.0 \\
\hline Laser & 818 & 3.23 & 477 & 58.3 & 746 & 91.2 \\
\hline Ultrasonic, Commercial & 2157 & 5.32 & 911 & 42.2 & 1675 & 77.7 \\
\hline Ultrasonic, Agency-built & 316 & 6.47 & 115 & 36.4 & 212 & 67.1 \\
\hline
\end{tabular}

The profilers with ultrasonic sensors were much less repeatable than the others, and do not appear to be acceptable for measuring IRI. Commercially built ultrasonic profilers performed much better than the agency-built profilers, but only a handful of ultrasonic profilers from Pennsylvania exhibited acceptable repeatability for network-level roughness measurement. The profilers with laser sensors were very often within 5 percent of the average, but did not pass the "reference device" test of repeating IRI within 2 percent consistently. Optical profilers performed the best, and were all sufficient for network-level applications.

The difference between the laser and optical profilers is most likely the sensor footprint. The diameter of the footprint of laser sensors ranges from 1 to 5 millimeters. The optical profilers use a rectangular footprint that is $6 \mathrm{~mm}$ long and by 150 millimeters wide. This large footprint means that the optical profilers are much less prone to variations caused by short features in the road that a laser profiler might capture in one run but miss in another, such as a narrow crack. The large footprint of the optical height sensor also averages out coarse texture, which is a physical form of anti-alias filtering. The width of the optical height sensor footprint probably also reduces variations in roughness caused by inconsistency in lateral positioning from run to run. With aggressive anti-alias filtering and spike detection, sensors with a very small footprint should be able to perform as well as the optical profilers did in the RPUG experiment.

\section{Ride Number}

The same statistics presented in table A-4 were also compiled for the RN. RN is defined as an index computed from profiles in two wheeltracks (4). Thus, only profilers that measured two wheeltracks are included in the analysis. In addition, bias in the analysis caused by the nonlinearity of the 0 to 5 scale was avoided by compiling statistics on the Pre-Transform Profile Index used to compute RN, rather than the RN itself. Table A-6 provides the results.

Two optical profilers (P05 and S08) and one laser profiler (P08) stood out as the most repeatable in measuring $\mathrm{RN}$, but some of the others were not even repeatable enough for network-level measurements. About half of the ultrasonic profilers measured RN with good repeatability, but they also measured $\mathrm{RN}$ with a significant downward bias. 
Table A-6. Repeatability of profilers in measurement of RN.

\begin{tabular}{|c|c|c|c|c|c|c|c|c|}
\hline \multirow{2}{*}{ Region } & \multirow{2}{*}{ Device } & \multirow{2}{*}{$\begin{array}{l}\text { Sensor } \\
\text { Type }\end{array}$} & \multirow{2}{*}{$\begin{array}{c}\text { Number of } \\
\text { Meas. }\end{array}$} & \multirow{2}{*}{$\begin{array}{c}\text { Std. Dev. } \\
(\%)\end{array}$} & \multicolumn{2}{|c|}{ Within 2 Percent } & \multicolumn{2}{|c|}{ Within 5 Percent } \\
\hline & & & & & (Count) & $(\%)$ & (Count) & $(\%)$ \\
\hline \multirow[t]{4}{*}{$\mathrm{M}$} & M02 & $\mathrm{U}$ & 79 & 7.45 & 25 & 31.6 & 51 & 64.6 \\
\hline & M03 & $\mathrm{L}$ & 58 & 5.96 & 32 & 55.2 & 45 & 77.6 \\
\hline & M05 & $\mathrm{U}$ & 80 & 28.03 & 8 & 10.0 & 24 & 30.0 \\
\hline & M06 & $\mathrm{O}$ & 100 & 4.73 & 62 & 62.0 & 90 & 90.0 \\
\hline \multirow[t]{6}{*}{$\mathrm{N}$} & N03 & $\mathrm{U}$ & 59 & 10.42 & 8 & 13.6 & 20 & 33.9 \\
\hline & N04 & $\mathrm{U}$ & 59 & 6.27 & 16 & 27.1 & 35 & 59.3 \\
\hline & N06 & $\mathrm{U}$ & 60 & 6.84 & 15 & 25.0 & 36 & 60.0 \\
\hline & N07 & $\mathrm{L}$ & 60 & 3.60 & 35 & 58.3 & 52 & 86.7 \\
\hline & N08 & $\mathrm{O}$ & 60 & 12.09 & 18 & 30.0 & 31 & 51.7 \\
\hline & N09 & $\mathrm{O}$ & 58 & 8.19 & 23 & 39.7 & 31 & 53.4 \\
\hline \multirow[t]{12}{*}{$\mathrm{P}$} & P01 & $\mathrm{L}$ & 80 & 2.79 & 56 & 70.0 & 73 & 91.3 \\
\hline & $\mathrm{P} 02$ & $\mathrm{U}$ & 80 & 3.67 & 39 & 48.8 & 68 & 85.0 \\
\hline & P03 & $\mathrm{U}$ & 80 & 4.23 & 35 & 43.8 & 68 & 85.0 \\
\hline & P04 & $\mathrm{L}$ & 80 & 2.87 & 51 & 63.8 & 75 & 93.8 \\
\hline & P05 & $\mathrm{O}$ & 80 & 1.76 & 64 & 80.0 & 79 & 98.8 \\
\hline & P06 & $\mathrm{U}$ & 78 & 35.64 & 4 & 5.1 & 27 & 34.6 \\
\hline & P07 & $\mathrm{O}$ & 80 & 9.55 & 28 & 35.0 & 50 & 62.5 \\
\hline & P08 & $\mathrm{L}$ & 73 & 2.36 & 48 & 65.8 & 70 & 95.9 \\
\hline & P73 & $\mathrm{U}$ & 79 & 14.19 & 34 & 43.0 & 65 & 82.3 \\
\hline & $\mathrm{P} 74$ & $\mathrm{U}$ & 79 & 4.00 & 39 & 49.4 & 66 & 83.5 \\
\hline & P75 & $\mathrm{U}$ & 80 & 3.59 & 31 & 38.8 & 64 & 80.0 \\
\hline & P76 & $\mathrm{U}$ & 80 & 2.61 & 47 & 58.8 & 75 & 93.8 \\
\hline \multirow[t]{5}{*}{$\bar{S}$} & S01 & $\mathrm{U}$ & 65 & 3.36 & 37 & 56.9 & 57 & 87.7 \\
\hline & S05 & $\mathrm{O}$ & 47 & 20.31 & 26 & 55.3 & 36 & 76.6 \\
\hline & S08 & $\mathrm{O}$ & 77 & 3.51 & 58 & 75.3 & 75 & 97.4 \\
\hline & S10 & $\mathrm{L}$ & 58 & 2.71 & 37 & 63.8 & 51 & 87.9 \\
\hline & S11 & U & 40 & 5.93 & 19 & 47.5 & 30 & 75.0 \\
\hline \multicolumn{2}{|c|}{$\begin{array}{l}\text { M - Mississippi } \\
\text { O - Optical }\end{array}$} & $\begin{array}{l}\mathrm{N}-\mathrm{Ne} \\
\mathrm{U}-\mathrm{Ul}\end{array}$ & sonic & $\begin{array}{l}\mathrm{P}-\mathrm{Pe} \\
\mathrm{L}-\mathrm{La}\end{array}$ & Ivania & & & \\
\hline
\end{tabular}

\section{AGREEMENT WITH THE DIPSTICK}

The accuracy of a profiling device is its ability to produce a result that is near the truth without bias. This is an illusive concept. No profiler measures the true profile in the sense that they are all limited to a finite waveband. For example, profilers do not measure topography or texture well, so some part of the true shape of the road is missed. On the other hand, it is possible for a profiler to measure the range of wavelengths of interest for computing IRI or RN correctly. The goal of this section is to present statistics that summarize the accuracy level of the profilers in the 1993 RPUG experiment.

All of the test sections in Mississippi, Pennsylvania, and South Dakota were measured with a Dipstick. The roughness values from these measurements are used as reference values for assessing the "accuracy" of the inertial profilers. All of the measurements covered the same longitudinal range as the Dipstick measurements. Although the lateral position of the profilers was not strictly controlled, paint marks were placed every 7.6 meters along the left wheeltrack of each section to help guide the drivers along a similar 
path. Since the experiment took place over a few months, changes in roughness of these sections with time may bias the roughness values.

The IRI values computed for all of the measurements in Mississippi, Pennsylvania, and South Dakota were normalized by a reference value from the Dipstick. For example, the ProRut measured eight sections ten times each. The IRI was computed for the left and right wheeltrack in each measurement, for a total of 160 roughness values. The bias between all of these roughness values and the corresponding value from the Dipstick were compiled into a histogram, shown in figure A-2.

The average of the 160 values in the figure is 9.4, which means that the ProRut measured IRI an average of 9.4 percent higher than the Dipstick. This is an overall estimate of the bias between the ProRut and the Dipstick. The average bias level represents the accuracy of the ProRut if the Dipstick is accepted as a reference. The RMS error was 12.3 percent. The RMS error penalizes a profiler for bias and scatter, so it delineates a combination of the accuracy and repeatability problems in a profiler. All profilers will have some level of RMS error. For network-level applications, a combination of no bias and an RMS error under 5 percent is preferred.

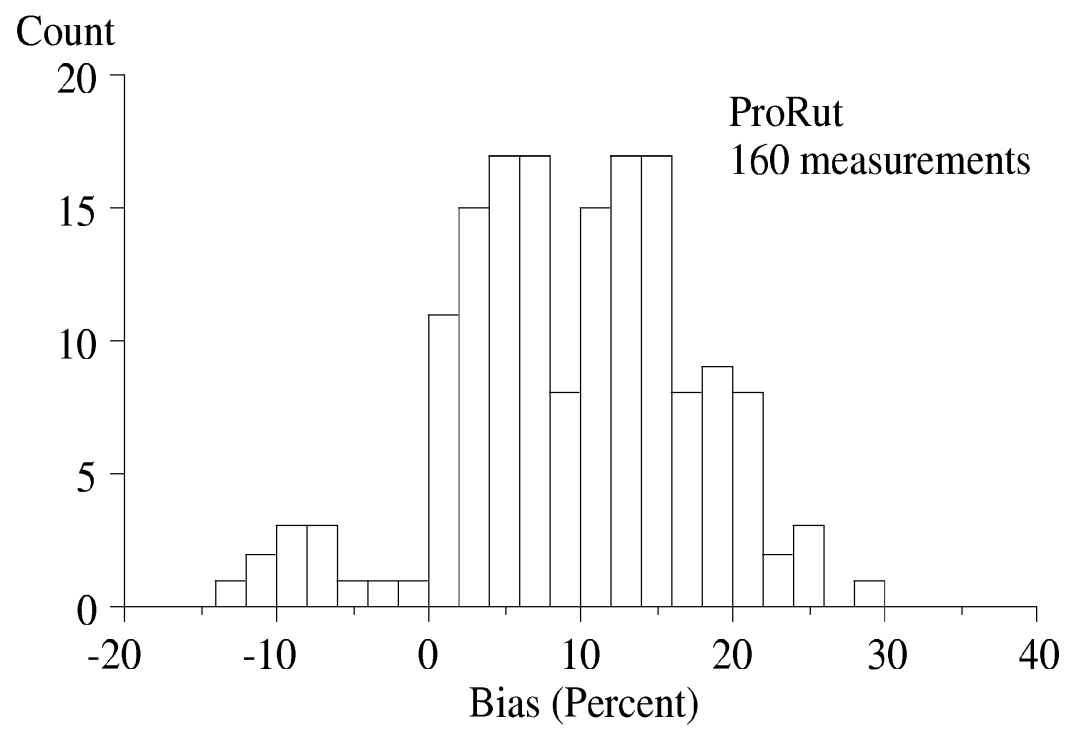

Figure A-2. Bias in IRI by the ProRut.

Bias errors can exist for several reasons: aliasing errors caused by narrow cracks or coarse surface texture, spikes in the sensor signals caused by the environment, variations in lateral tracking that consistently place a profiler on a path other than the one measured by the reference device, or changes in the road surface between the day of the reference measurement and the day of the other tests. The RMS error should include all of the factors that confound profiler measurement, including the factors just listed and everything that degrades the repeatability of a set of measurements.

IRI values from all of the profilers in Mississippi, Pennsylvania, and South Dakota were compared to Dipstick values as a means of characterizing each profiler's bias and RMS error level. The results are listed in table A-7. The results should not be interpreted too precisely, since not every profiler made the same set of measurements. Not all of the profilers within a region measured all of the sections exactly ten times. Some profilers, 
usually the South Dakota type, measured only the left wheeltrack. Each region had a unique set of sections, so only comparisons of major trends should be made across all of the regions. Nevertheless, some of the trends are so strong that they are meaningful.

Very few devices stood out as agreeing with the Dipstick measurements very well. The few promising numbers in table A-7 are listed in bold. The bias and RMS error of these devices was heavily linked to the sensor type. Figures A-3 through A-6 show the histograms for all measurements by four broad types of profiler: (1) agency-built ultrasonic, (2) commercially built ultrasonic, (3) laser, and (4) optical. Table A-8 also provides summary statistics. All of the histograms are shown on the same scale for comparison. The optical profilers had the lowest bias and RMS error, followed by the laser profilers. As described in the section on repeatability, the large footprint of the optical sensors is probably the reason optical profilers in the RPUG study generally agreed with the Dipstick more closely than laser profilers.

Table A-7. Agreement to Dipstick of profilers in measurement of IRI.

\begin{tabular}{|c|c|c|c|c|c|c|c|}
\hline Region & Device & $\begin{array}{l}\text { Sensor } \\
\text { Type }\end{array}$ & $\begin{array}{c}\text { Number of } \\
\text { Meas. }\end{array}$ & $\begin{array}{r}\text { Bias } \\
(\%) \\
\end{array}$ & $\begin{array}{c}\text { RMS Error } \\
(\%)\end{array}$ & $\begin{array}{l}\text { Within } 5 \\
\text { (Count) }\end{array}$ & $\begin{array}{l}\text { Percent } \\
(\%)\end{array}$ \\
\hline \multirow[t]{5}{*}{ M } & M01 & $\mathrm{U}$ & 80 & 11.4 & 14.8 & 19 & 23.8 \\
\hline & M02 & $\mathrm{U}$ & 158 & 21.3 & 26.9 & 21 & 13.3 \\
\hline & M03 & $\mathrm{L}$ & 116 & 5.1 & 10.8 & 38 & 32.8 \\
\hline & M05 & $\mathrm{U}$ & 160 & 9.3 & 15.9 & 46 & 28.8 \\
\hline & M06 & $\mathrm{O}$ & 200 & 4.7 & 8.4 & 77 & 38.5 \\
\hline \multirow[t]{12}{*}{$\mathrm{P}$} & P01 & $\mathrm{L}$ & 160 & 9.4 & 12.3 & 36 & 22.5 \\
\hline & $\mathrm{P} 02$ & $\mathrm{U}$ & 160 & 30.1 & 34.8 & 0 & 0.0 \\
\hline & P03 & $\mathrm{U}$ & 160 & 27.3 & 32.3 & 0 & 0.0 \\
\hline & P04 & $\mathrm{L}$ & 160 & 12.0 & 16.3 & 37 & 23.1 \\
\hline & P05 & $\mathrm{O}$ & 160 & 11.0 & 14.9 & 27 & 16.9 \\
\hline & P06 & $\mathrm{U}$ & 156 & 32.4 & 39.0 & 0 & 0.0 \\
\hline & P07 & $\mathrm{O}$ & 160 & 11.3 & 13.3 & 19 & 11.9 \\
\hline & P08 & $\mathrm{L}$ & 146 & 9.5 & 11.5 & 40 & 27.4 \\
\hline & P73 & $\mathrm{U}$ & 159 & 25.7 & 31.7 & 4 & 2.5 \\
\hline & P74 & $\mathrm{U}$ & 158 & 23.7 & 27.9 & 5 & 3.2 \\
\hline & P75 & $\mathrm{U}$ & 160 & 21.1 & 26.8 & 13 & 8.1 \\
\hline & P76 & $\mathrm{U}$ & 160 & 22.6 & 28.3 & 3 & 1.9 \\
\hline \multirow[t]{10}{*}{ S } & S01 & $\mathrm{U}$ & 130 & 44.8 & 62.3 & 23 & 17.7 \\
\hline & S02 & $\mathrm{U}$ & 80 & 18.9 & 29.2 & 24 & 30.0 \\
\hline & S03 & $\mathrm{U}$ & 80 & 42.4 & 68.5 & 27 & 33.8 \\
\hline & S04 & $\mathrm{U}$ & 77 & 51.1 & 70.4 & 18 & 23.4 \\
\hline & S05 & $\mathrm{O}$ & 94 & -0.3 & 7.6 & 70 & 74.5 \\
\hline & S06 & $\mathrm{U}$ & 79 & 50.6 & 71.7 & 16 & 20.3 \\
\hline & S08 & $\mathrm{O}$ & 154 & 2.9 & 7.3 & 83 & 53.9 \\
\hline & S10 & $\mathrm{L}$ & 116 & 13.7 & 19.1 & 33 & 28.4 \\
\hline & $\mathrm{S} 11$ & $\mathrm{U}$ & 80 & 36.0 & 51.0 & 8 & 10.0 \\
\hline & $\mathrm{S} 12$ & $\mathrm{U}$ & 80 & 51.8 & 77.3 & 19 & 23.8 \\
\hline \multicolumn{2}{|c|}{$\begin{array}{l}\text { M - Mississippi } \\
\text { O - Optical }\end{array}$} & $\begin{array}{l}\text { - Nevada } \\
\text { - Ultrasc }\end{array}$ & & $\begin{array}{l}\text { P - Pen } \\
L-\text { Lase }\end{array}$ & ania & \multicolumn{2}{|c|}{ S - South Dakota } \\
\hline
\end{tabular}




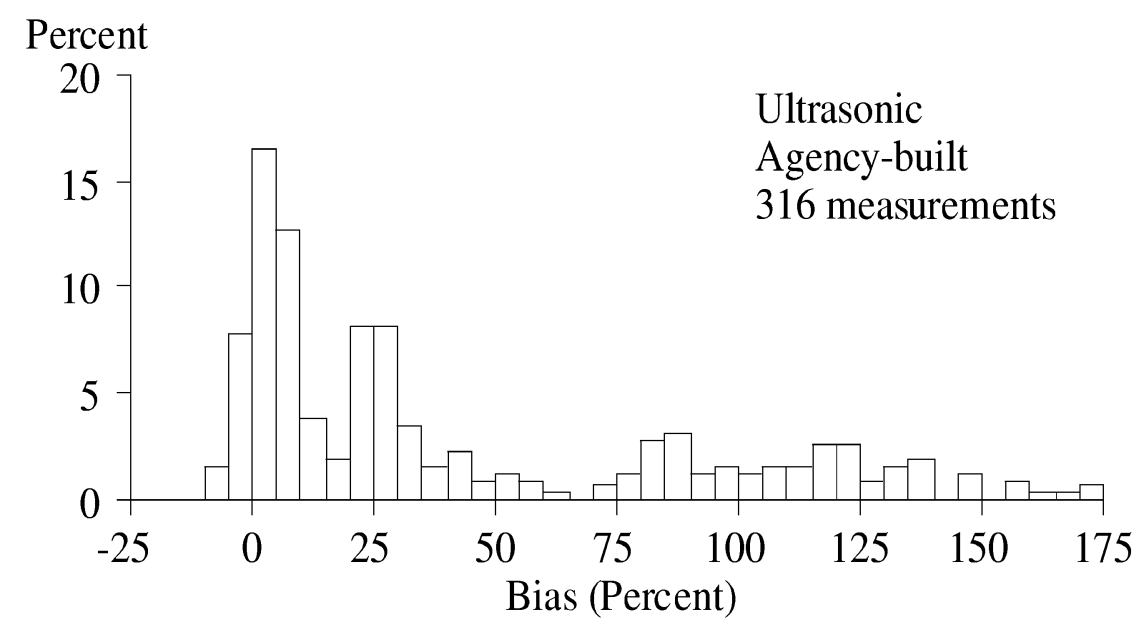

Figure A-3. Bias in agency-built ultrasonic profilers from the RPUG.

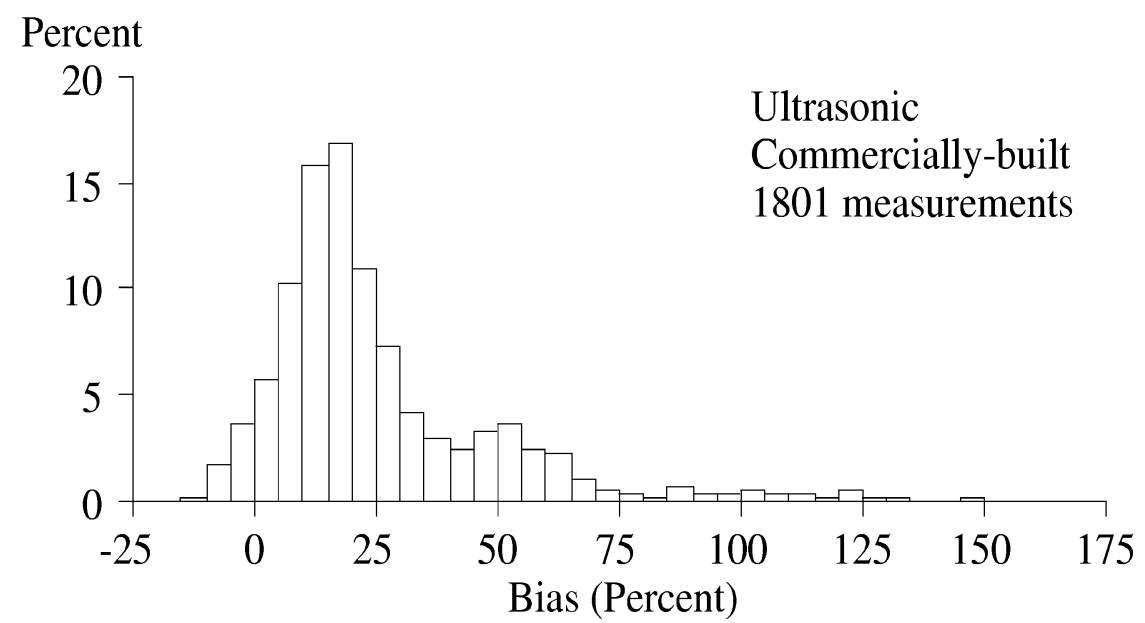

Figure A-4. Bias in commercial ultrasonic profilers from the RPUG.

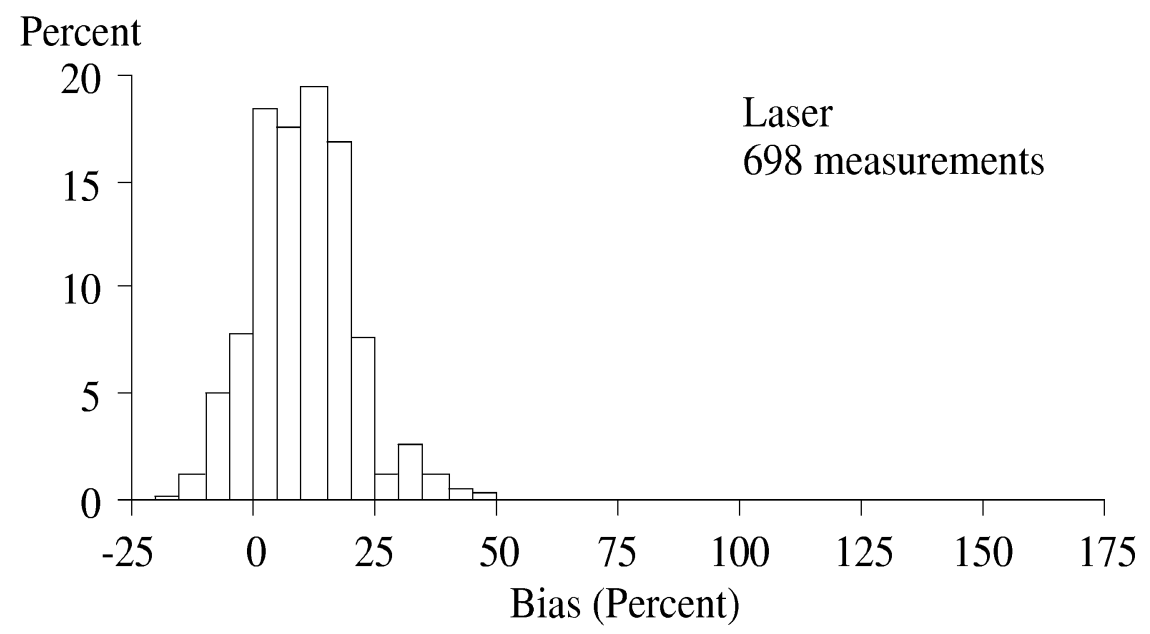

Figure A-5. Bias in laser profilers from the RPUG. 


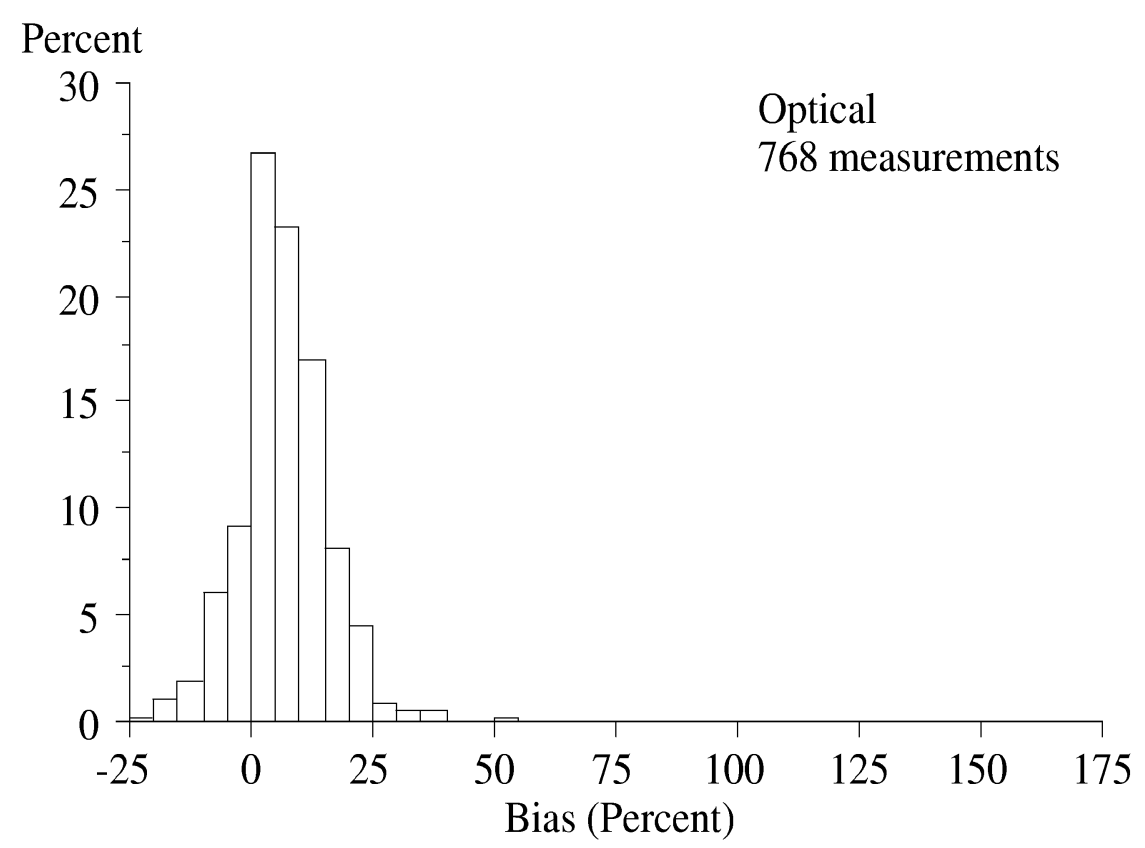

Figure A-6. Bias in optical profilers from the RPUG.

The scatter and bias of the laser and optical profilers compared to the Dipstick measurements are most likely caused by lateral tracking variations, sensing of short features that the Dipstick ignores, the lack of aggressive measures to avoid aliasing errors, and problems inherent in using noncontact sensors in an uncontrolled environment at high speed.

The ultrasonic profilers, both agency-built and commercial, measured IRI with huge bias and scatter. In contrast to the laser and optical sensors, ultrasonic sensors are not sufficient for the job of measuring IRI. Some of the commercial ultrasonic profilers performed well on most sections, but horribly on sections with coarse surface texture. This is why the histograms in figure A-3 and A-4 extend so far to the right. If IRI values are to be compared from agency to agency or year to year, ultrasonic sensors must be replaced.

Table A-8. Agreement to the Dipstick of profiler types in IRI measurement.

\begin{tabular}{|c|c|c|c|c|c|}
\hline Profiler Type & $\begin{array}{c}\text { Number of } \\
\text { Meas. }\end{array}$ & $\begin{array}{l}\text { Bias } \\
(\%)\end{array}$ & $\begin{array}{c}\text { RMS Error } \\
(\%)\end{array}$ & $\begin{array}{l}\text { Within } \\
\text { (Count) }\end{array}$ & $\begin{array}{c}\text { ercent } \\
(\%)\end{array}$ \\
\hline Optical & 768 & 6.4 & 10.9 & 276 & 35.9 \\
\hline Laser & 698 & 10.0 & 14.2 & 184 & 26.4 \\
\hline Ultrasonic, Commercial & 1801 & 26.1 & 36.4 & 169 & 9.4 \\
\hline Ultrasonic, Agency-built & 316 & 43.0 & 65.0 & 77 & 24.4 \\
\hline
\end{tabular}

\section{SURFACE TEXTURE}

Coarse surface macrotexture has the potential to cause an upward bias in roughness. For example, on a pavement with a fresh chip seal height sensors with a small footprint may detect the top of a piece of protruding aggregate in one sample and miss the aggregate in another. If the sample interval is too large or a profiler operates without anti-aliasing filters, the texture could erroneously appear in the final profile as deviations with a long 
enough wavelength to affect the IRI or RN. Laser sensors sample fast enough to allow surface texture to be recognized and averaged out using anti-alias filters. Optical height sensors have a footprint so large that coarse texture is probably averaged out.

Ultrasonic sensors have a footprint that is 50 to 100 millimeters in diameter. This footprint is large enough to average out texture, but ultrasonic height sensors do not work this way. They register a reading as soon as the reflected acoustic wave is first detected, so they actually detect the highest point within the footprint. There is no way to average these deviations out, because a reading can only be taken about 3 or 4 times per meter at highway speed. This causes a major bias in roughness measurement on roads with coarse texture.

For example, sections 3 and 4 in Pennsylvania were asphalt surfaces with chip seals. These two sections had very coarse surface texture compared to the others. Table A-9 lists the texture depth from ASTM sand patch tests of sections 1 through 8 in Pennsylvania. All of the profilers with ultrasonic sensors measured IRI with an extreme bias on sections 3 and 4. Figure A-7 shows a histogram of all of the measurements by ultrasonic profilers in Pennsylvania. There is a group of measurements with a bias around 20 percent, but a second, smaller group with a bias of about 55 percent. The group with a bias around 55 percent is mostly measurements of sections 3 and 4 .

Table A-9. Bias in profilers by section in Pennsylvania.

\begin{tabular}{|c|c|c|c|c|c|c|c|c|}
\hline Surface Type & \multicolumn{2}{|c|}{$\begin{array}{c}\text { AC Without } \\
\text { Chip Seal }\end{array}$} & \multicolumn{2}{|c|}{$\begin{array}{c}\text { AC With Chip } \\
\text { Seal }\end{array}$} & \multicolumn{4}{|c|}{ Portland Cement Concrete } \\
\hline Section Number & 1 & 2 & 3 & 4 & 5 & 6 & 7 & 8 \\
\hline Macrotexture Depth $(\mathrm{mm})$ & 0.65 & 0.53 & 1.78 & 1.39 & 0.66 & 0.58 & 0.53 & 0.69 \\
\hline Bias, Optical Profilers (\%) & 16.5 & -1.6 & 6.1 & 8.1 & 9.6 & 12.4 & 19.5 & 18.3 \\
\hline Bias, Laser Profilers (\%) & 15.3 & 5.9 & 4.8 & 5.0 & 3.2 & 11.6 & 16.8 & 19.9 \\
\hline Bias, Ultrasonic Profilers (\%) & 18.3 & 18.7 & 54.5 & 46.1 & 22.1 & 12.3 & 16.2 & 20.3 \\
\hline
\end{tabular}

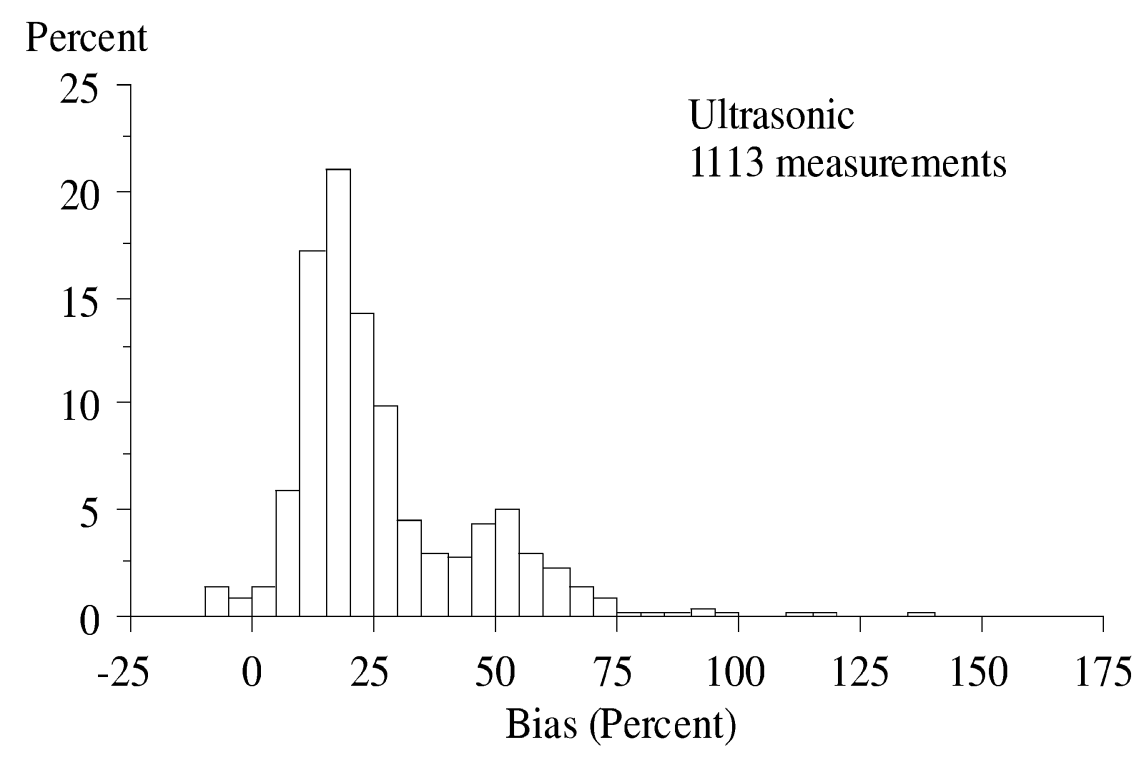

Figure A-7. Bias in ultrasonic profilers from the RPUG in Pennsylvania.

Table A-9 summarizes the bias in IRI compared to the Dipstick on each section in Pennsylvania by height sensor type. The results for the other six sections are also listed for 
comparison. The ultrasonic profilers had extreme difficulty with sections 3 and 4 . In fact, none of their measurements agree with the Dipstick within 5 percent. Their performance is much better on the other sections, but still not acceptable.

The profilers with laser and optical sensors actually agree with the Dipstick more closely on sections 3 and 4 than the others. Coarse macrotexture of the kind typical of a chip seal apparently does not cause systematic errors in these profilers. However, all of the laser and optical profilers showed the highest bias on sections 7 and 8. A likely explanation for the elevated roughness is that the laser and optical sensors registered roughness at opened joints (or cracks) that the Dipstick did not. This may also explain the bias in IRI on section 1, which was very rough and probably included narrow forms of distress like cracks that the Dipstick would ignore.

Table A-10 summarizes the bias in IRI compared to the Dipstick on each section in South Dakota by height sensor type. Sections 2, 3, and 4 in South Dakota all had chip seals, and all have high values of macrotexture depth. Profilers with ultrasonic sensors exhibited an extreme bias on these sections. The optical profilers agreed reasonably well with the Dipstick on all of the sections, but the laser profiler did not. Only one laser profiler participated in the study in South Dakota. Most of the bias in its measurements come from large upward spikes that are not caused by coarse texture.

Table A-10. Bias in profilers by section in South Dakota.

\begin{tabular}{|l|c|c|c|c|c|c|c|c|}
\hline Surface Type & \multicolumn{4}{|c|}{ Asphalt Concrete } & \multicolumn{3}{c|}{ Portland Cement Concrete } \\
\cline { 1 - 1 } Section Num. & 1 & 2 & 3 & 4 & 5 & 6 & 7 & 8 \\
\hline Macrotexture Depth (mm) & 1.08 & 1.42 & 1.31 & 1.33 & 0.78 & 0.33 & 0.67 & 0.38 \\
\hline Bias, Optical Profilers (\%) & -5.3 & -1.0 & -1.2 & 2.1 & 9.3 & 3.4 & 4.2 & 3.5 \\
\hline Bias, Laser Profilers (\%) & 5.6 & 20.3 & 10.6 & 28.2 & 12.9 & -1.5 & 6.7 & - \\
\hline Bias, Ultrasonic Profilers (\%) & 4.3 & 104.5 & 59.7 & 107.6 & 17.2 & 5.9 & 16.9 & 11.8 \\
\hline
\end{tabular}

\section{OPERATING SPEED}

Most of the profilers listed in table A-2 performed ten or more measurements of each section: five measurements at a speed near $80 \mathrm{kph}$ (called higher speed repeats) and five more at a speed near $64 \mathrm{kph}$ (called lower speed repeats). In a few isolated cases, measurements were made at speeds of $72 \mathrm{kph}$ and $56 \mathrm{kph}$ instead. This matrix of runs was intended to reveal any bias in roughness measurement caused by modest variations in operating speed. Tables A-11 through A-14 list the ratio of the Mean Roughness Index (MRI) measured at the higher speed to the MRI measured at the lower speed. (MRI is the average of the IRI from the left and right wheeltrack.) Each value in the tables represents the average of the higher speed repeats divided by the average of the lower speed repeats on a particular section by a particular profiler. In most cases, exactly five measurements of each section at each speed were made. A value is only listed if at least four measurements at each speed were available. A table is provided for each region, since each region used a distinct set of sections. (In other words, section 1 in South Dakota is not the same as section 1 in Pennsylvania, etc.) 
Table A-11. Speed sensitivity of profilers in Mississippi.

Average MRI at high speed/Average MRI at low speed Asphalt Sections Concrete Sections

\begin{tabular}{|c|c|c|c|c|c|c|c|c|}
\cline { 1 - 1 } & \multicolumn{3}{c|}{ Asphalt Sections } & \multicolumn{4}{c|}{ Concrete Sections } \\
Device & 1 & 2 & 3 & 4 & 5 & 6 & 7 & 8 \\
\hline M02 & 1.06 & 1.08 & 1.05 & 1.01 & 1.06 & 0.97 & 0.97 & 1.01 \\
\hline M03 & - & - & 0.97 & - & 1.02 & 1.04 & - & 0.99 \\
\hline M05 & 1.03 & 0.96 & 1.01 & 1.03 & 1.02 & 1.00 & 1.05 & 1.14 \\
\hline M06 & 1.02 & 1.01 & 0.99 & 1.03 & 1.00 & 0.99 & 1.01 & 1.01 \\
\hline
\end{tabular}

Table A-12. Speed sensitivity of profilers in Nevada.

\begin{tabular}{|c|c|c|c|c|c|c|}
\multicolumn{1}{c|}{} & \multicolumn{3}{c|}{ Average MRI at high speed/Average MRI at low speed } \\
\cline { 1 - 1 } Asphalt Sections & 1 & 2 & 3 & 4 & 5 & 6 \\
\hline N03 & 1.06 & 1.03 & 1.06 & 1.12 & 1.10 & 1.01 \\
\hline N04 & 1.08 & 1.07 & 1.15 & 1.01 & 1.04 & 1.01 \\
\hline N06 & 1.11 & 1.11 & 1.03 & 1.01 & 1.00 & 0.99 \\
\hline N07 & 0.98 & 0.97 & 0.96 & 1.01 & 0.99 & 0.99 \\
\hline N08 & 1.00 & 1.01 & 0.97 & 1.05 & 1.02 & 1.00 \\
\hline N09 & 0.97 & 1.01 & 1.03 & 1.07 & 0.97 & 1.00 \\
\hline
\end{tabular}

Table A-13. Speed sensitivity of profilers in Pennsylvania.

\begin{tabular}{|c|c|c|c|c|c|c|c|c|}
\multicolumn{1}{c|}{} & \multicolumn{7}{c|}{ Average MRI at high speed/Average MRI at low speed } \\
Anevice & 1 & 2 & 3 & 4 & 5 & 6 & 7 & 8 \\
\hline P01 & 1.00 & 1.01 & 0.99 & 0.98 & 0.99 & 0.97 & 1.05 & 0.99 \\
\hline P02 & 1.04 & 1.06 & 0.96 & $\mathbf{1 . 1 2}$ & 1.04 & 0.98 & 1.02 & 1.00 \\
\hline P03 & 1.05 & 0.98 & 0.95 & $\mathbf{1 . 0 8}$ & 1.05 & 0.99 & 0.99 & 1.03 \\
\hline P04 & 0.99 & 0.92 & 0.98 & 0.93 & 0.97 & 1.00 & 0.98 & 0.95 \\
\hline P05 & 1.05 & 1.00 & 1.00 & 1.00 & 1.02 & 1.03 & 1.04 & 1.03 \\
\hline P06 & 1.02 & 0.97 & 1.02 & $\mathbf{1 . 2 6}$ & 1.03 & 1.02 & 1.04 & 0.98 \\
\hline P07 & 1.00 & 1.07 & 1.01 & 1.00 & 1.00 & 0.99 & 0.98 & 1.01 \\
\hline P08 & 1.04 & 1.05 & 1.02 & 0.94 & 1.01 & 1.02 & 1.01 & 1.02 \\
\hline P73 & 0.99 & 1.01 & 0.98 & $\mathbf{1 . 1 2}$ & 0.98 & 0.97 & 1.00 & 1.01 \\
\hline P74 & 1.00 & 1.00 & 0.99 & $\mathbf{1 . 0 8}$ & 1.01 & 0.99 & 0.98 & 1.00 \\
\hline P75 & 1.02 & 1.03 & 0.99 & $\mathbf{1 . 0 8}$ & 0.99 & 0.98 & 0.98 & 0.99 \\
\hline P76 & 0.99 & 0.98 & 1.00 & $\mathbf{1 . 0 3}$ & 1.00 & 1.00 & 1.00 & 0.98 \\
\hline
\end{tabular}

Table A-14. Speed sensitivity of profilers in South Dakota.

\begin{tabular}{|c|c|c|c|c|c|c|c|c|}
\cline { 5 - 9 } \multicolumn{1}{c|}{} & \multicolumn{8}{c|}{ Average MRI at high speed/Average MRI at low speed } \\
\cline { 1 - 5 } Device & 1 & 2 & 3 & 4 & 5 & 6 & 7 & 8 \\
\hline S01 & - & 0.98 & 1.05 & 1.02 & 1.02 & - & 1.01 & - \\
\hline S08 & 1.01 & 0.99 & 1.01 & 1.02 & 1.01 & 0.99 & 1.02 & 1.00 \\
\hline S10 & 1.01 & 0.99 & 0.99 & 0.99 & 0.98 & - & - & - \\
\hline
\end{tabular}


Table A-15 summarizes the results for each profiler organized by sensor type. The table lists the average of the values given for all sections in tables A-11 through A-14 and the minimum and maximum. Keep in mind that profilers from different regions encountered different sections and not all profilers covered all sections, so only major trends are likely to have significant implications about the effect of operating speed. Very few of these devices showed an overall bias with operating speed that was more significant than the scatter they exhibit within a given speed. (That is, the average listed in table A-15 rarely accounts for most of the standard deviation listed in table A-4, and the scatter within repeats at the two speeds overlap each other.)

Table A-15. Summary of trends in MRI with operating speed.

\begin{tabular}{|c|c|c|c|c|}
\hline Sensor Type & Device & Average & Minimum & Maximum \\
\hline \multirow[t]{6}{*}{ Laser } & M03 & 1.00 & 0.97 & 1.04 \\
\hline & N07 & 0.98 & 0.96 & 1.01 \\
\hline & P01 & 1.00 & 0.97 & 1.05 \\
\hline & P04 & 0.97 & 0.92 & 1.00 \\
\hline & P08 & 1.01 & 0.94 & 1.05 \\
\hline & $\mathrm{S} 10$ & 0.99 & 0.98 & 1.01 \\
\hline \multirow[t]{6}{*}{ Optical } & M06 & 1.01 & 0.99 & 1.03 \\
\hline & N08 & 1.01 & 0.97 & 1.05 \\
\hline & N09 & 1.01 & 0.97 & 1.07 \\
\hline & P05 & 1.02 & 1.00 & 1.05 \\
\hline & P07 & 1.01 & 0.98 & 1.07 \\
\hline & S08 & 1.01 & 0.99 & 1.02 \\
\hline \multirow[t]{13}{*}{ Ultrasonic } & M02 & 1.03 & 0.97 & 1.08 \\
\hline & M05 & 1.03 & 0.96 & 1.14 \\
\hline & N03 & 1.06 & 1.01 & 1.12 \\
\hline & N04 & 1.06 & 1.01 & 1.15 \\
\hline & N06 & 1.04 & 0.99 & 1.11 \\
\hline & P02 & 1.03 & 0.96 & 1.12 \\
\hline & P03 & 1.01 & 0.95 & 1.08 \\
\hline & P06 & 1.04 & 0.97 & 1.26 \\
\hline & P73 & 1.01 & 0.98 & 1.12 \\
\hline & P74 & 1.01 & 0.98 & 1.08 \\
\hline & P75 & 1.01 & 0.98 & 1.08 \\
\hline & P76 & 1.00 & 0.98 & 1.03 \\
\hline & S01 & 1.02 & 0.98 & 1.05 \\
\hline
\end{tabular}

In general, the profilers with ultrasonic sensors measured higher MRI values at the higher speed. This is because a sensor error that drives up the roughness is more likely to occur at higher speed. This explains why the averages in table A-15 for ultrasonic sensors are all greater than one, although the trend is weak. One example that stands out (if for no other reason, because they are in bold type) is that all of the profilers with ultrasonic sensors measured MRI values that were significantly higher at the higher speed on Pennsylvania section 4. (See table A-14.) This is not the section with the highest macrotexture depth, but its texture did seem most problematic to profilers with ultrasonic sensors. 
A few other weak trends exist with speed, but most of them are not systematic. In most of the cases of extreme values in tables A-11 through A-14, a single anomalous value from one measurement skewed the average for one of the speeds, rather than a systematic bias in all repeats. The only other case of a systematic bias with speed was exhibited by profiler P04. It produced lower MRI at the higher speed on all sections. The explanation for this is not known.

Table A-16 shows a summary for trends in RN with operating speed compiled in the same manner as MRI in table A-15. Very few of the profilers are speed sensitive. A few of the ultrasonic profilers appeared speed sensitive on sections of coarse macrotexture, but the $\mathrm{RN}$ values were so far off at both speeds that the trend is not worth examination.

Table A-16. Summary of trends in RN with operating speed.

\begin{tabular}{|c|c|c|c|c|}
\hline Sensor Type & Device & Average & Minimum & Maximum \\
\hline Laser & M03 & 1.01 & 0.99 & 1.06 \\
\cline { 2 - 5 } & N07 & 1.00 & 1.00 & 1.01 \\
\cline { 2 - 5 } & P01 & 0.99 & 0.96 & 1.00 \\
\cline { 2 - 5 } & P04 & 1.00 & 0.96 & 1.02 \\
\cline { 2 - 5 } & P08 & 0.99 & 0.97 & 1.01 \\
\cline { 2 - 5 } & S10 & 1.02 & 0.99 & 1.04 \\
\hline Optical & M06 & 1.01 & 0.98 & 1.09 \\
\cline { 2 - 5 } & N08 & 0.98 & 0.89 & 1.10 \\
\cline { 2 - 5 } & N09 & 0.95 & 0.90 & 1.00 \\
\cline { 2 - 5 } & P05 & 0.99 & 0.97 & 1.00 \\
\cline { 2 - 5 } & P07 & 1.00 & 0.95 & 1.09 \\
\cline { 2 - 5 } & S08 & 1.01 & 1.00 & 1.02 \\
\hline Ultrasonic & M02 & 0.99 & 0.91 & 1.05 \\
\cline { 2 - 5 } & M05 & 1.01 & 0.85 & 1.13 \\
\cline { 2 - 5 } & N03 & 0.93 & 0.88 & 1.00 \\
\cline { 2 - 5 } & N04 & 0.98 & 0.93 & 1.04 \\
\cline { 2 - 5 } & N06 & 0.97 & 0.92 & 1.00 \\
\cline { 2 - 5 } & P02 & 1.00 & 0.96 & 1.04 \\
\cline { 2 - 5 } & P03 & 0.99 & 0.96 & 1.02 \\
\cline { 2 - 5 } & P06 & 0.87 & 0.50 & 1.03 \\
\cline { 2 - 5 } & P73 & 0.98 & 0.73 & 1.04 \\
\cline { 2 - 5 } & P74 & 1.00 & 0.97 & 1.02 \\
\cline { 2 - 5 } & P75 & 1.00 & 0.96 & 1.05 \\
\cline { 2 - 5 } & P76 & 1.00 & 0.98 & 1.02 \\
\cline { 2 - 5 } & S01 & 0.98 & 0.92 & 1.00 \\
\hline
\end{tabular}

\section{REFERENCES}

1. Karamihas, S. M., et. al., "Guidelines for Longitudinal Pavement Profile Measurement. Appendix C." National Cooperative Highway Research Program Report 454 (1999). 
2. Perera, R. W. and Kohn, S. D., "Road Profiler User Group Fifth Annual Meeting. Road Profiler Data Analysis and Correlation." Soil and Materials Engineers, Inc., Research Report No. 92-30 (1994) 87 p.

3. Huft, D. L. "Description and Evaluation of the South Dakota Road Profiler." Federal Highway Administration, FHWA-DP-89-072-002 (1989) 159 p.

4. Sayers, M. W. and Karamihas, S. M., "Interpretation of Road Roughness Profile Data." Federal Highway Administration, FHWA/RD-96/101 (1996) 177 p. 


\section{Appendix B: Cross Correlation Results, 1993 RPUG Study}

This appendix lists the results of cross correlation analysis performed on all of the profile measurements from the 1993 RPUG study. Details of the study are provided in Appendix A, and are documented elsewhere. (1) Ratings of agreement between profilers and repeatability of each device are listed for four wavebands:

1. IRI Filter: The output of the IRI algorithm. This is a slope profile with frequency weighting determined by the quarter car filter using the Golden Car parameters.

2. Long Wavelengths: The slope profile, passed through a four-pole Butterworth filter with cutoff wavelengths of 8 and 40 meters.

3. Medium Wavelengths: The slope profile, passed through a four-pole Butterworth filter with cutoff wavelengths of 1.6 and 8 meters.

4. Short Wavelengths: The slope profile, passed through a four-pole Butterworth filter with cutoff wavelengths of 0.32 and 1.6 meters.

Cross correlation of IRI filter output emphasizes content in the profiles that is relevant to the accumulation of IRI. The other three filters were included to help diagnose the source of disagreement between profiles by isolating each waveband. All of the cross correlation values were calculated after longitudinal distance measurement offset was removed, so the profiles cover the same pavement. On the other hand, linear distance measurement error was not corrected. Errors in distance measurement as small as a percent reduce correlation, particularly in the short wavelength range.

Two hundred forty tables of cross correlation values are provided, covering each type of filter and sixty individual wheeltracks (up to eight sites in four regions, left and right). Table B-1 lists all of the devices covered by the analysis and the number of measurements they made on each site. Tables B-2 through B-241 cover all possible combinations of device on all sites for all four wavebands.

For each combination of filter and site, all of the measurements from a given device are compared to all of the measurements from the other. The result is an average correlation level that constitutes a single entry in one table. Each table entry provides the average correlation level for every combination of measurements by the appropriate pair of devices. For example, on the left wheeltrack of Mississippi site 1, profiler M01 and M02 each made ten profile measurements. Thus, one hundred comparisons are possible, and the table entry is the average of one hundred cross correlation values.

Note that the two devices are compared twice. In the first comparison, M01 is deemed the "reference device," and the measurements from device M02 are compared to them. This means that the sample interval from device M01 is retained, and the measurements from device M02 are interpolated to obtain a one-to-one correspondence. In the second comparison, profiles from device M02 are treated as the reference, and profiles from device M01 are interpolated to match them. The two values are usually similar, except when the 
short wavelengths are compared. This is because the short wavelengths are affected most by sample interval.

Not all entrees are the average of one hundred values. When fewer measurements were made by one of the devices, it reduced the number of possible comparisons. Several devices did not measure the right wheeltrack, so they are omitted from the appropriate tables.

The individual table entrees provide a rating of the ability of one device to reproduce the measurements of another in a given waveband. Agreement to the Dipstick was thought of as a profiler's "accuracy" at the time of the experiment. Although the Dipstick measurements can not be considered a perfect reflection of the true profile, it is the only reference device that participated in the study. The Dipstick only measured each wheeltrack once. Thus, the majority of listings for agreement to the Dipstick are the average of ten correlation values.

The diagonal entrees in the tables provide a rating of repeatability, because they are the average of the correlation levels that result for all combinations of repeat measurements by the same device. (Ten repeat measurements yield forty-five possible comparisons, so these entrees are usually the average of forty-five correlation values.) No values are listed for repeatability of the Dipstick, because no repeat measurements were made.

Note also that correlation level is expressed on a scale that ranges from -100 to 100 , rather than -1 to 1 . This is done for ease of interpretation. There are several reasons why the correlation levels are lower than 100. Differences in height sensor technology, sample interval, and profile computation method degrade the level of agreement between devices. Most devices measured the site at different times and on different dates. The correlation levels are therefore affected by changes in roughness with time, including the trend toward higher roughness as the pavement ages, and cyclic changes caused by the weather and climate. Agreement is, of course, also degraded by measurement error. Agreement and repeatability are both degraded by lateral wander of the profilers. The RPUG study did include special provisions to help high-speed profilers maintain a consistent lateral position during the measurements, but this can never be completely controlled. Gentle lateral wander usually only affects measurement of short wavelengths.

Several relevant trends appear in these data that may have helped diagnose some of the measurement problems discovered in the 1993 RPUG study. For example, the lack of reciprocity in some of the cross correlation values suggests that the sample interval was insufficient for the waveband of interest. (Reciprocity is obtained when comparisons of one device to another are not sensitive to which device is chosen as a correlation reference. The choice of a reference is important because the other device's profile is interpolated to match its sample interval.) In particular, the lack of reciprocity observed when the IRI filter is used in the cross correlation procedure is evidence that the sample interval was not sufficient for computation of the IRI. The extremely poor correlation in the short wavelength range also indicates that the shorter sample interval was needed, or that filtering was not done properly for very short wavelengths. Indeed, the profilers with ultrasonic sensors used no low-pass filtering at all. 
Further, the diversity in cross correlation level of the same profiler on different sites provides insight into which sites challenge each profiler the most. The most obvious example of this was the degraded correlation in the medium wavelengths, short wavelengths and IRI filter on coarse textured sites by profilers with ultrasonic sensors.

Table B-1. Coverage of each site in the 1993 RPUG experiment.

\begin{tabular}{|c|c|c|c|c|c|c|c|c|c|c|c|c|}
\hline \multirow[t]{2}{*}{ Region } & \multirow[t]{2}{*}{ Inst. \# } & \multirow{2}{*}{$\begin{array}{c}\text { Sensor } \\
\text { Type }\end{array}$} & \multirow[t]{2}{*}{ Tracks } & \multicolumn{9}{|c|}{ Number of Measurements at Each Site } \\
\hline & & & & All & 1 & 2 & 3 & 4 & 5 & 6 & 7 & 8 \\
\hline \multirow[t]{6}{*}{$\bar{M}$} & MDS & 1 & B & 8 & 1 & 1 & 1 & 1 & 1 & 1 & 1 & 1 \\
\hline & M01 & $\mathrm{U}$ & $\mathrm{L}$ & 80 & 10 & 10 & 10 & 10 & 10 & 10 & 10 & 10 \\
\hline & M02 & $\mathrm{U}$ & B & 79 & 10 & 10 & 10 & 10 & 10 & 10 & 9 & 10 \\
\hline & M03 & $\mathrm{L}$ & $\mathrm{B}$ & 58 & 8 & 4 & 9 & 8 & 10 & 9 & 0 & 10 \\
\hline & M05 & $\mathrm{U}$ & $\mathrm{B}$ & 80 & 10 & 10 & 10 & 10 & 10 & 10 & 10 & 10 \\
\hline & M06 & $\mathrm{O}$ & $\mathrm{B}$ & 100 & 10 & 10 & 14 & 16 & 12 & 14 & 10 & 14 \\
\hline \multirow{7}{*}{$\mathrm{N}$} & NDS & I & B & 8 & 1 & 1 & 1 & 1 & 1 & 1 & - & - \\
\hline & N03 & $\mathrm{U}$ & B & 59 & 10 & 10 & 10 & 10 & 9 & 10 & - & - \\
\hline & N04 & $\mathrm{U}$ & $\mathrm{B}$ & 60 & 10 & 10 & 10 & 10 & 10 & 10 & - & - \\
\hline & N06 & $\mathrm{U}$ & B & 60 & 10 & 10 & 10 & 10 & 10 & 10 & - & - \\
\hline & N07 & $\mathrm{L}$ & B & 61 & 10 & 10 & 10 & 10 & 11 & 10 & - & - \\
\hline & N08 & $\mathrm{O}$ & B & 60 & 10 & 10 & 10 & 10 & 10 & 10 & - & - \\
\hline & N09 & $\mathrm{O}$ & B & 58 & 10 & 10 & 10 & 10 & 8 & 10 & - & - \\
\hline \multirow[t]{13}{*}{$\bar{P}$} & PDS & $\bar{I}$ & $\bar{B}$ & 8 & 1 & 1 & 1 & 1 & 1 & 1 & 1 & 1 \\
\hline & P01 & $\mathrm{L}$ & B & 80 & 10 & 10 & 10 & 10 & 10 & 10 & 10 & 10 \\
\hline & $\mathrm{P} 02$ & $\mathrm{U}$ & B & 80 & 10 & 10 & 10 & 10 & 10 & 10 & 10 & 10 \\
\hline & $\mathrm{P} 03$ & $\mathrm{U}$ & B & 80 & 10 & 10 & 10 & 10 & 10 & 10 & 10 & 10 \\
\hline & P04 & $\mathrm{L}$ & B & 80 & 10 & 10 & 10 & 10 & 10 & 10 & 10 & 10 \\
\hline & P05 & $\mathrm{O}$ & B & 80 & 10 & 10 & 10 & 10 & 10 & 10 & 10 & 10 \\
\hline & P06 & $\mathrm{U}$ & B & 78 & 9 & 10 & 10 & 10 & 10 & 10 & 10 & 9 \\
\hline & P07 & $\mathrm{O}$ & B & 80 & 10 & 10 & 10 & 10 & 10 & 10 & 10 & 10 \\
\hline & P08 & $\mathrm{L}$ & B & 74 & 9 & 8 & 9 & 10 & 9 & 10 & 9 & 10 \\
\hline & P73 & $\mathrm{U}$ & B & 80 & 10 & 10 & 10 & 10 & 10 & 10 & 10 & 10 \\
\hline & P74 & $\mathrm{U}$ & B & 79 & 10 & 10 & 10 & 10 & 10 & 10 & 9 & 10 \\
\hline & P75 & $\mathrm{U}$ & B & 80 & 10 & 10 & 10 & 10 & 10 & 10 & 10 & 10 \\
\hline & P76 & $\mathrm{U}$ & B & 80 & 10 & 10 & 10 & 10 & 10 & 10 & 10 & 10 \\
\hline \multirow[t]{11}{*}{$\bar{S}$} & SDS & I & B & 8 & 1 & 1 & 1 & 1 & 1 & 1 & 1 & 1 \\
\hline & S01 & U & B & 65 & 5 & 10 & 10 & 10 & 10 & 5 & 10 & 5 \\
\hline & S02 & $\mathrm{U}$ & $\mathrm{L}$ & 80 & 10 & 10 & 10 & 10 & 10 & 10 & 10 & 10 \\
\hline & S03 & $\mathrm{U}$ & $\mathrm{L}$ & 80 & 10 & 10 & 10 & 10 & 10 & 10 & 10 & 10 \\
\hline & S04 & $\mathrm{U}$ & $\mathrm{L}$ & 77 & 10 & 10 & 10 & 10 & 7 & 10 & 10 & 10 \\
\hline & S05 & $\mathrm{O}$ & B & 47 & 6 & 8 & 4 & 7 & 2 & 6 & 10 & 4 \\
\hline & S06 & $\mathrm{U}$ & $\mathrm{L}$ & 79 & 9 & 10 & 10 & 10 & 10 & 10 & 10 & 10 \\
\hline & S08 & $\mathrm{O}$ & B & 77 & 10 & 9 & 10 & 10 & 10 & 9 & 9 & 10 \\
\hline & S10 & $\mathrm{L}$ & B & 58 & 10 & 10 & 10 & 10 & 10 & 4 & 4 & 0 \\
\hline & S11 & $\mathrm{U}$ & B & 40 & 5 & 5 & 5 & 5 & 5 & 5 & 5 & 5 \\
\hline & S12 & $\mathrm{U}$ & $\mathrm{L}$ & 80 & 10 & 10 & 10 & 10 & 10 & 10 & 10 & 10 \\
\hline \multirow{2}{*}{\multicolumn{3}{|c|}{$\begin{array}{l}\text { M - Mississippi } \\
\text { I - Inclinometer }\end{array}$}} & & & & $\overline{\text { Penn }}$ & 17 & & & & & \\
\hline & & & Optical & & & Ult & & & & Las & & \\
\hline \multicolumn{2}{|l|}{$\begin{array}{l}\text { 1 - Inclin } \\
\text { L - Left }\end{array}$} & & Both (L & an & ht) & & & & & & & \\
\hline
\end{tabular}


Table B-2. Mississippi Site 1 Left, IRI Filter.

\begin{tabular}{|c|c|c|c|c|c|c|}
\hline Correlation & \multicolumn{6}{|c|}{ Correlated Device } \\
Reference & 01 & 02 & 03 & 05 & 06 & DS \\
\hline 01 & 78 & 54 & 69 & 65 & 69 & 70 \\
\hline 02 & 57 & 81 & 52 & 48 & 68 & 65 \\
\hline 03 & 71 & 52 & 79 & 67 & 70 & 76 \\
\hline 05 & 67 & 47 & 67 & 61 & 66 & 68 \\
\hline 06 & 72 & 69 & 70 & 66 & 88 & 83 \\
\hline DS & 74 & 65 & 78 & 67 & 84 & - \\
\hline
\end{tabular}

Table B-3. Mississippi Site 1 Left, Long Wavelengths.

\begin{tabular}{|c|c|c|c|c|c|c|}
\hline Correlation & \multicolumn{6}{|c|}{ Correlated Device } \\
Reference & 01 & 02 & 03 & 05 & 06 & DS \\
\hline 01 & 95 & 83 & 75 & 86 & 95 & 81 \\
\hline 02 & 84 & 97 & 88 & 76 & 86 & 90 \\
\hline 03 & 76 & 88 & 97 & 73 & 78 & 95 \\
\hline 05 & 85 & 75 & 73 & 85 & 86 & 76 \\
\hline 06 & 95 & 85 & 78 & 87 & $\mathbf{9 9}$ & 84 \\
\hline DS & 81 & 90 & 95 & 76 & 84 & - \\
\hline
\end{tabular}

Table B-4. Mississippi Site 1 Left, Medium Wavelengths.

\begin{tabular}{|c|c|c|c|c|c|c|}
\hline Correlation & \multicolumn{6}{|c|}{ Correlated Device } \\
Reference & 01 & 02 & 03 & 05 & 06 & DS \\
\hline 01 & 71 & 39 & 60 & 52 & 57 & 60 \\
\hline 02 & 45 & 71 & 38 & 33 & 55 & 50 \\
\hline 03 & 64 & 38 & 73 & 60 & 63 & 67 \\
\hline 05 & 59 & 34 & 61 & 53 & 57 & 61 \\
\hline 06 & 62 & 56 & 63 & 56 & 83 & 77 \\
\hline DS & 69 & 51 & 68 & 58 & 79 & - \\
\hline
\end{tabular}

Table B-5. Mississippi Site 1 Left, Short Wavelengths.

\begin{tabular}{|c|c|c|c|c|c|c|}
\hline Correlation & \multicolumn{6}{|c|}{ Correlated Device } \\
Reference & 01 & 02 & 03 & 05 & 06 & DS \\
\hline 01 & 45 & 9 & 11 & 8 & 7 & 15 \\
\hline 02 & 16 & 39 & 8 & 7 & 8 & 9 \\
\hline 03 & 21 & 13 & 42 & 23 & 12 & 26 \\
\hline 05 & 21 & 11 & 18 & 19 & 11 & 18 \\
\hline 06 & 22 & 15 & 13 & 12 & 50 & 29 \\
\hline DS & 31 & 12 & 17 & 14 & 24 & - \\
\hline
\end{tabular}


Table B-6. Mississippi Site 1 Right, IRI Filter.

\begin{tabular}{|c|c|c|c|c|c|}
\hline Correlation & \multicolumn{5}{|c|}{ Correlated Device } \\
Reference & 02 & 03 & 05 & 06 & DS \\
\hline 02 & 80 & 51 & 51 & 65 & 60 \\
\hline 03 & 49 & 90 & 78 & 73 & 79 \\
\hline 05 & 48 & 77 & 75 & 76 & 69 \\
\hline 06 & 61 & 72 & 74 & $\mathbf{9 5}$ & 72 \\
\hline DS & 58 & 83 & 74 & 76 & - \\
\hline
\end{tabular}

Table B-7. Mississippi Site 1 Right, Long Wavelengths.

\begin{tabular}{|c|c|c|c|c|c|}
\hline Correlation & \multicolumn{5}{|c|}{ Correlated Device } \\
Reference & 02 & 03 & 05 & 06 & DS \\
\hline 02 & 97 & 85 & 71 & 84 & 87 \\
\hline 03 & 85 & 98 & 72 & 79 & 96 \\
\hline 05 & 70 & 72 & 92 & 82 & 72 \\
\hline 06 & 83 & 79 & 83 & 99 & 82 \\
\hline DS & 86 & 96 & 73 & 82 & - \\
\hline
\end{tabular}

Table B-8. Mississippi Site 1 Right, Medium Wavelengths.

\begin{tabular}{|c|c|c|c|c|c|}
\hline Correlation & \multicolumn{5}{|c|}{ Correlated Device } \\
Reference & 02 & 03 & 05 & 06 & DS \\
\hline 02 & 73 & 43 & 42 & 56 & 50 \\
\hline 03 & 39 & 88 & 75 & 67 & 71 \\
\hline 05 & 39 & 75 & 68 & 68 & 64 \\
\hline 06 & 49 & 65 & 61 & 93 & 65 \\
\hline DS & 47 & 78 & 70 & 72 & - \\
\hline
\end{tabular}

Table B-9. Mississippi Site 1 Right, Short Wavelengths.

\begin{tabular}{|c|c|c|c|c|c|}
\hline $\begin{array}{c}\text { Correlation } \\
\text { Reference }\end{array}$ & \multicolumn{5}{|c|}{ Correlated Device } \\
\hline 02 & 42 & 11 & 9 & 10 & 7 \\
\hline 03 & 11 & 63 & 28 & 14 & 23 \\
\hline 05 & 10 & 31 & 21 & 17 & 18 \\
\hline 06 & 10 & 15 & 14 & 68 & 19 \\
\hline DS & 9 & 33 & 21 & 29 & - \\
\hline
\end{tabular}


Table B-10. Mississippi Site 2 Left, IRI Filter.

\begin{tabular}{|c|c|c|c|c|c|c|}
\hline Correlation & \multicolumn{6}{|c|}{ Correlated Device } \\
Reference & 01 & 02 & 03 & 05 & 06 & DS \\
\hline 01 & 71 & 34 & 57 & 62 & 75 & 64 \\
\hline 02 & 37 & 49 & 34 & 32 & 42 & 36 \\
\hline 03 & 57 & 32 & 71 & 56 & 61 & 65 \\
\hline 05 & 62 & 30 & 56 & 62 & 65 & 63 \\
\hline 06 & 75 & 39 & 60 & 65 & 92 & 72 \\
\hline DS & 61 & 33 & 64 & 60 & 71 & - \\
\hline
\end{tabular}

Table B-11. Mississippi Site 2 Left, Long Wavelengths.

\begin{tabular}{|c|c|c|c|c|c|c|}
\hline Correlation & \multicolumn{5}{|c|}{ Correlated Device } \\
Reference & 01 & 02 & 03 & 05 & 06 & DS \\
\hline 01 & 98 & 78 & 72 & 94 & 92 & 74 \\
\hline 02 & 78 & 95 & 87 & 76 & 86 & 92 \\
\hline 03 & 71 & 87 & 99 & 70 & 79 & 91 \\
\hline 05 & 94 & 76 & 70 & 93 & 88 & 73 \\
\hline 06 & 92 & 86 & 79 & 88 & 99 & 83 \\
\hline DS & 74 & 92 & 91 & 73 & 83 & - \\
\hline
\end{tabular}

Table B-12. Mississippi Site 2 Left, Medium Wavelengths.

\begin{tabular}{|c|c|c|c|c|c|c|}
\hline Correlation & \multicolumn{6}{|c|}{ Correlated Device } \\
Reference & 01 & 02 & 03 & 05 & 06 & DS \\
\hline 01 & 54 & 16 & 45 & 39 & 60 & 51 \\
\hline 02 & 19 & 33 & 15 & 15 & 24 & 18 \\
\hline 03 & 46 & 13 & 48 & 43 & 49 & 45 \\
\hline 05 & 42 & 13 & 42 & 43 & 44 & 47 \\
\hline 06 & 60 & 21 & 47 & 41 & 82 & 60 \\
\hline DS & 50 & 15 & 44 & 40 & 58 & - \\
\hline
\end{tabular}

Table B-13. Mississippi Site 2 Left, Short Wavelengths.

\begin{tabular}{|c|c|c|c|c|c|c|}
\hline Correlation & \multicolumn{6}{|c|}{ Correlated Device } \\
\hline Reference & 01 & 02 & 03 & 05 & 06 & DS \\
\hline 01 & 33 & 3 & 7 & 4 & 8 & 8 \\
\hline 02 & 6 & 19 & 8 & 7 & 8 & 8 \\
\hline 03 & 14 & 7 & 14 & 11 & 10 & 12 \\
\hline 05 & 13 & 6 & 11 & 16 & 9 & 8 \\
\hline 06 & 28 & 6 & 8 & 6 & 33 & 10 \\
\hline DS & 20 & 5 & 9 & 6 & 12 & - \\
\hline
\end{tabular}


Table B-14. Mississippi Site 2 Right, IRI Filter.

\begin{tabular}{|c|c|c|c|c|c|}
\hline Correlation & \multicolumn{5}{|c|}{ Correlated Device } \\
Reference & 02 & 03 & 05 & 06 & DS \\
\hline 02 & 55 & 44 & 40 & 48 & 50 \\
\hline 03 & 42 & 90 & 69 & 75 & 81 \\
\hline 05 & 38 & 71 & 68 & 73 & 67 \\
\hline 06 & 46 & 75 & 70 & $\mathbf{9 4}$ & 76 \\
\hline DS & 47 & 86 & 69 & 80 & - \\
\hline
\end{tabular}

Table B-15. Mississippi Site 2 Right, Long Wavelengths.

\begin{tabular}{|c|c|c|c|c|c|}
\hline Correlation & \multicolumn{5}{|c|}{ Correlated Device } \\
Reference & 02 & 03 & 05 & 06 & DS \\
\hline 02 & 95 & 92 & 71 & 84 & 91 \\
\hline 03 & 92 & 99 & 69 & 80 & 94 \\
\hline 05 & 71 & 69 & 95 & 85 & 70 \\
\hline 06 & 84 & 81 & 85 & 99 & 83 \\
\hline DS & 91 & 94 & 70 & 83 & - \\
\hline
\end{tabular}

Table B-16. Mississippi Site 2 Right, Medium Wavelengths.

\begin{tabular}{|c|c|c|c|c|c|}
\hline Correlation & \multicolumn{5}{|c|}{ Correlated Device } \\
Reference & 02 & 03 & 05 & 06 & DS \\
\hline 02 & 31 & 22 & 20 & 27 & 27 \\
\hline 03 & 20 & 81 & 56 & 69 & 69 \\
\hline 05 & 18 & 63 & 54 & 54 & 59 \\
\hline 06 & 24 & 70 & 47 & 87 & 64 \\
\hline DS & 23 & 78 & 53 & 73 & - \\
\hline
\end{tabular}

Table B-17. Mississippi Site 2 Right, Short Wavelengths.

\begin{tabular}{|c|c|c|c|c|c|}
\hline Correlation & \multicolumn{5}{|c|}{ Correlated Device } \\
Reference & 02 & 03 & 05 & 06 & DS \\
\hline 02 & 14 & 8 & 7 & 9 & 10 \\
\hline 03 & 6 & 36 & 15 & 11 & 19 \\
\hline 05 & 7 & 25 & 18 & 10 & 14 \\
\hline 06 & 6 & 11 & 6 & 50 & 19 \\
\hline DS & 8 & 27 & 10 & 23 & - \\
\hline
\end{tabular}


Table B-18. Mississippi Site 3 Left, IRI Filter.

\begin{tabular}{|c|c|c|c|c|c|c|}
\hline Correlation & \multicolumn{6}{|c|}{ Correlated Device } \\
Reference & 01 & 02 & 03 & 05 & 06 & DS \\
\hline 01 & 52 & 31 & 47 & 31 & 43 & 55 \\
\hline 02 & 34 & 44 & 28 & 29 & 33 & 37 \\
\hline 03 & 47 & 29 & 78 & 48 & 67 & 62 \\
\hline 05 & 34 & 30 & 49 & 47 & 50 & 46 \\
\hline 06 & 49 & 35 & 69 & 51 & 82 & 57 \\
\hline DS & 50 & 33 & 57 & 42 & 54 & - \\
\hline
\end{tabular}

Table B-19. Mississippi Site 3 Left, Long Wavelengths.

\begin{tabular}{|c|c|c|c|c|c|c|}
\hline Correlation & \multicolumn{6}{|c|}{ Correlated Device } \\
Reference & 01 & 02 & 03 & 05 & 06 & DS \\
\hline 01 & 94 & 69 & 54 & 78 & 91 & 64 \\
\hline 02 & 70 & 94 & 85 & 62 & 74 & 87 \\
\hline 03 & 55 & 85 & 92 & 51 & 59 & 87 \\
\hline 05 & 78 & 62 & 51 & 75 & 79 & 57 \\
\hline 06 & 92 & 74 & 60 & 79 & 99 & 69 \\
\hline DS & 65 & 86 & 87 & 57 & 69 & - \\
\hline
\end{tabular}

Table B-20. Mississippi Site 3 Left, Medium Wavelengths.

\begin{tabular}{|c|c|c|c|c|c|c|}
\hline Correlation & \multicolumn{6}{|c|}{ Correlated Device } \\
Reference & 01 & 02 & 03 & 05 & 06 & DS \\
\hline 01 & 47 & 20 & 42 & 24 & 33 & 51 \\
\hline 02 & 26 & 33 & 20 & 22 & 24 & 28 \\
\hline 03 & 43 & 21 & 76 & 43 & 61 & 54 \\
\hline 05 & 28 & 24 & 45 & 43 & 42 & 40 \\
\hline 06 & 40 & 27 & 63 & 44 & 79 & 49 \\
\hline DS & 44 & 22 & 49 & 35 & 45 & - \\
\hline
\end{tabular}

Table B-21. Mississippi Site 3 Left, Short Wavelengths.

\begin{tabular}{|c|c|c|c|c|c|c|}
\hline $\begin{array}{c}\text { Correlation } \\
\text { Reference }\end{array}$ & \multicolumn{6}{|c|}{ Correlated Device } \\
\hline 01 & 31 & 02 & 03 & 05 & 06 & DS \\
\hline 02 & 8 & 17 & 8 & 8 & 7 & 13 \\
\hline 03 & 20 & 8 & 63 & 21 & 28 & 27 \\
\hline 05 & 13 & 9 & 19 & 20 & 13 & 26 \\
\hline 06 & 17 & 12 & 35 & 21 & 64 & 30 \\
\hline DS & 27 & 7 & 20 & 16 & 15 & - \\
\hline
\end{tabular}


Table B-22. Mississippi Site 3 Right, IRI Filter.

\begin{tabular}{|c|c|c|c|c|c|}
\hline Correlation & \multicolumn{5}{|c|}{ Correlated Device } \\
Reference & 02 & 03 & 05 & 06 & DS \\
\hline 02 & 55 & 31 & 32 & 39 & 33 \\
\hline 03 & 28 & 76 & 38 & 64 & 65 \\
\hline 05 & 30 & 40 & 39 & 53 & 37 \\
\hline 06 & 36 & 65 & 52 & 89 & 49 \\
\hline DS & 30 & 62 & 34 & 47 & - \\
\hline
\end{tabular}

Table B-23. Mississippi Site 3 Right, Long Wavelengths.

\begin{tabular}{|c|c|c|c|c|c|}
\hline Correlation & \multicolumn{5}{|c|}{ Correlated Device } \\
Reference & 02 & 03 & 05 & 06 & DS \\
\hline 02 & 91 & 84 & 57 & 65 & 79 \\
\hline 03 & 83 & 97 & 54 & 61 & 92 \\
\hline 05 & 57 & 54 & 76 & 78 & 55 \\
\hline 06 & 65 & 61 & 78 & 99 & 64 \\
\hline DS & 79 & 92 & 55 & 64 & - \\
\hline
\end{tabular}

Table B-24. Mississippi Site 3 Right, Medium Wavelengths.

\begin{tabular}{|c|c|c|c|c|c|}
\hline Correlation & \multicolumn{5}{|c|}{ Correlated Device } \\
Reference & 02 & 03 & 05 & 06 & DS \\
\hline 02 & 45 & 23 & 25 & 32 & 25 \\
\hline 03 & 19 & 72 & 32 & 58 & 59 \\
\hline 05 & 22 & 34 & 33 & 46 & 30 \\
\hline 06 & 27 & 60 & 44 & 86 & 46 \\
\hline DS & 20 & 55 & 27 & 42 & - \\
\hline
\end{tabular}

Table B-25. Mississippi Site 3 Right, Short Wavelengths.

\begin{tabular}{|c|c|c|c|c|c|}
\hline Correlation & \multicolumn{5}{|c|}{ Correlated Device } \\
Reference & 02 & 03 & 05 & 06 & DS \\
\hline 02 & 22 & 8 & 9 & 12 & 9 \\
\hline 03 & 5 & 62 & 15 & 25 & 32 \\
\hline 05 & 8 & 17 & 12 & 17 & 14 \\
\hline 06 & 9 & 27 & 14 & 68 & 33 \\
\hline DS & 5 & 25 & 8 & 19 & - \\
\hline
\end{tabular}


Table B-26. Mississippi Site 4 Left, IRI Filter.

\begin{tabular}{|c|c|c|c|c|c|c|}
\hline Correlation & \multicolumn{6}{|c|}{ Correlated Device } \\
Reference & 01 & 02 & 03 & 05 & 06 & DS \\
\hline 01 & 53 & 35 & 41 & 32 & 56 & 58 \\
\hline 02 & 38 & 60 & 27 & 36 & 60 & 51 \\
\hline 03 & 42 & 28 & 74 & 48 & 58 & 47 \\
\hline 05 & 36 & 39 & 51 & 57 & 52 & 38 \\
\hline 06 & 56 & 56 & 53 & 47 & 90 & 73 \\
\hline DS & 52 & 45 & 46 & 34 & 71 & - \\
\hline
\end{tabular}

Table B-27. Mississippi Site 4 Left, Long Wavelengths.

\begin{tabular}{|c|c|c|c|c|c|c|}
\hline Correlation & \multicolumn{6}{|c|}{ Correlated Device } \\
Reference & 01 & 02 & 03 & 05 & 06 & DS \\
\hline 01 & 90 & 60 & 48 & 82 & 87 & 44 \\
\hline 02 & 60 & 95 & 59 & 60 & 68 & 91 \\
\hline 03 & 48 & 58 & 73 & 51 & 56 & 53 \\
\hline 05 & 82 & 60 & 51 & 88 & 84 & 46 \\
\hline 06 & 87 & 68 & 56 & 85 & 98 & 51 \\
\hline DS & 45 & 91 & 53 & 46 & 51 & - \\
\hline
\end{tabular}

Table B-28. Mississippi Site 4 Left, Medium Wavelengths.

\begin{tabular}{|c|c|c|c|c|c|c|}
\hline Correlation & \multicolumn{6}{|c|}{ Correlated Device } \\
Reference & 01 & 02 & 03 & 05 & 06 & DS \\
\hline 01 & 46 & 25 & 41 & 24 & 48 & 52 \\
\hline 02 & 30 & 50 & 26 & 28 & 54 & 43 \\
\hline 03 & 43 & 27 & 72 & 44 & 55 & 46 \\
\hline 05 & 30 & 32 & 49 & 49 & 46 & 32 \\
\hline 06 & 48 & 45 & 52 & 39 & 88 & 71 \\
\hline DS & 46 & 34 & 43 & 27 & 69 & - \\
\hline
\end{tabular}

Table B-29. Mississippi Site 4 Left, Short Wavelengths.

\begin{tabular}{|c|c|c|c|c|c|c|}
\hline Correlation & \multicolumn{6}{|c|}{ Correlated Device } \\
Reference & 01 & 02 & 03 & 05 & 06 & DS \\
\hline 01 & 28 & 4 & 9 & 4 & 8 & 10 \\
\hline 02 & 9 & 28 & 8 & 8 & 17 & 10 \\
\hline 03 & 16 & 10 & 42 & 21 & 25 & 21 \\
\hline 05 & 12 & 13 & 23 & 25 & 23 & 17 \\
\hline 06 & 22 & 13 & 20 & 12 & 77 & 30 \\
\hline DS & 24 & 7 & 13 & 8 & 38 & - \\
\hline
\end{tabular}


Table B-30. Mississippi Site 4 Right, IRI Filter.

\begin{tabular}{|c|c|c|c|c|c|}
\hline Correlation & \multicolumn{5}{|c|}{ Correlated Device } \\
Reference & 02 & 03 & 05 & 06 & DS \\
\hline 02 & 66 & 28 & 40 & 54 & 52 \\
\hline 03 & 26 & 84 & 48 & 56 & 68 \\
\hline 05 & 36 & 47 & 47 & 63 & 52 \\
\hline 06 & 49 & 54 & 59 & $\mathbf{9 5}$ & 73 \\
\hline DS & 46 & 67 & 51 & 77 & - \\
\hline
\end{tabular}

Table B-31. Mississippi Site 4 Right, Long Wavelengths.

\begin{tabular}{|c|c|c|c|c|c|}
\hline Correlation & \multicolumn{5}{|c|}{ Correlated Device } \\
Reference & 02 & 03 & 05 & 06 & DS \\
\hline 02 & 95 & 79 & 62 & 74 & 91 \\
\hline 03 & 80 & 91 & 59 & 67 & 74 \\
\hline 05 & 63 & 59 & 88 & 82 & 46 \\
\hline 06 & 75 & 68 & 82 & 99 & 55 \\
\hline DS & 91 & 74 & 47 & 55 & - \\
\hline
\end{tabular}

Table B-32. Mississippi Site 4 Right, Medium Wavelengths.

\begin{tabular}{|c|c|c|c|c|c|}
\hline Correlation & \multicolumn{5}{|c|}{ Correlated Device } \\
Reference & 02 & 03 & 05 & 06 & DS \\
\hline 02 & 59 & 24 & 33 & 47 & 45 \\
\hline 03 & 20 & 82 & 41 & 52 & 63 \\
\hline 05 & 29 & 44 & 39 & 54 & 46 \\
\hline 06 & 39 & 50 & 47 & 94 & 65 \\
\hline DS & 37 & 63 & 42 & 72 & - \\
\hline
\end{tabular}

Table B-33. Mississippi Site 4 Right, Short Wavelengths.

\begin{tabular}{|c|c|c|c|c|c|}
\hline Correlation & \multicolumn{5}{|c|}{ Correlated Device } \\
Reference & 02 & 03 & 05 & 06 & DS \\
\hline 02 & 30 & 9 & 7 & 15 & 8 \\
\hline 03 & 7 & 52 & 11 & 16 & 23 \\
\hline 05 & 9 & 17 & 14 & 19 & 15 \\
\hline 06 & 10 & 13 & 10 & 84 & 25 \\
\hline DS & 6 & 19 & 8 & 31 & - \\
\hline
\end{tabular}


Table B-34. Mississippi Site 5 Left, IRI Filter.

\begin{tabular}{|c|c|c|c|c|c|c|}
\hline Correlation & \multicolumn{6}{|c|}{ Correlated Device } \\
Reference & 01 & 02 & 03 & 05 & 06 & DS \\
\hline 01 & 87 & 49 & 78 & 76 & 74 & 78 \\
\hline 02 & 55 & 82 & 53 & 39 & 76 & 64 \\
\hline 03 & 82 & 50 & $\mathbf{9 4}$ & 82 & 80 & 86 \\
\hline 05 & 80 & 37 & 82 & 87 & 68 & 76 \\
\hline 06 & 78 & 70 & 79 & 66 & $\mathbf{9 7}$ & 86 \\
\hline DS & 80 & 58 & 84 & 73 & 86 & - \\
\hline
\end{tabular}

Table B-35. Mississippi Site 5 Left, Long Wavelengths.

\begin{tabular}{|c|c|c|c|c|c|c|}
\hline $\begin{array}{c}\text { Correlation } \\
\text { Reference }\end{array}$ & \multicolumn{5}{|c|}{ Correlated Device } \\
\hline 01 & 91 & 02 & 03 & 05 & 06 & DS \\
\hline 02 & 79 & 99 & 74 & 81 & 94 & 81 \\
\hline 03 & 74 & 80 & 98 & 67 & 81 & 83 \\
\hline 05 & 81 & 66 & 71 & 88 & 83 & 75 \\
\hline 06 & 93 & 81 & 78 & 83 & 99 & 85 \\
\hline DS & 81 & 83 & 95 & 76 & 85 & - \\
\hline
\end{tabular}

Table B-36. Mississippi Site 5 Left, Medium Wavelengths.

\begin{tabular}{|c|c|c|c|c|c|c|}
\hline Correlation & \multicolumn{6}{|c|}{ Correlated Device } \\
Reference & 01 & 02 & 03 & 05 & 06 & DS \\
\hline 01 & 85 & 39 & 74 & 68 & 66 & 70 \\
\hline 02 & 46 & 77 & 42 & 30 & 69 & 54 \\
\hline 03 & 79 & 42 & 93 & 79 & 76 & 81 \\
\hline 05 & 77 & 29 & 79 & 84 & 60 & 71 \\
\hline 06 & 73 & 64 & 73 & 56 & 97 & 81 \\
\hline DS & 79 & 49 & 79 & 66 & 84 & - \\
\hline
\end{tabular}

Table B-37. Mississippi Site 5 Left, Short Wavelengths.

\begin{tabular}{|c|c|c|c|c|c|c|}
\hline Correlation & \multicolumn{6}{|c|}{ Correlated Device } \\
\hline Reference & 01 & 02 & 03 & 05 & 06 & DS \\
\hline 01 & 69 & 7 & 26 & 17 & 13 & 20 \\
\hline 02 & 12 & 48 & 9 & 9 & 17 & 14 \\
\hline 03 & 46 & 11 & 78 & 38 & 22 & 35 \\
\hline 05 & 41 & 11 & 46 & 61 & 12 & 32 \\
\hline 06 & 32 & 19 & 22 & 9 & 85 & 35 \\
\hline DS & 36 & 15 & 45 & 27 & 47 & - \\
\hline
\end{tabular}


Table B-38. Mississippi Site 5 Right, IRI Filter.

\begin{tabular}{|c|c|c|c|c|c|}
\hline Correlation & \multicolumn{5}{|c|}{ Correlated Device } \\
Reference & 02 & 03 & 05 & 06 & DS \\
\hline 02 & 84 & 55 & 43 & 73 & 57 \\
\hline 03 & 52 & $\mathbf{9 7}$ & 86 & 81 & 79 \\
\hline 05 & 40 & 85 & 89 & 72 & 70 \\
\hline 06 & 67 & 80 & 69 & $\mathbf{9 7}$ & 81 \\
\hline DS & 52 & 76 & 66 & 80 & - \\
\hline
\end{tabular}

Table B-39. Mississippi Site 5 Right, Long Wavelengths.

\begin{tabular}{|c|c|c|c|c|c|}
\hline Correlation & \multicolumn{5}{|c|}{ Correlated Device } \\
Reference & 02 & 03 & 05 & 06 & DS \\
\hline 02 & 96 & 82 & 69 & 79 & 77 \\
\hline 03 & 81 & 98 & 74 & 81 & 90 \\
\hline 05 & 68 & 74 & 93 & 86 & 80 \\
\hline 06 & 79 & 80 & 87 & 98 & 83 \\
\hline DS & 76 & 90 & 80 & 82 & - \\
\hline
\end{tabular}

Table B-40. Mississippi Site 5 Right, Medium Wavelengths.

\begin{tabular}{|c|c|c|c|c|c|}
\hline Correlation & \multicolumn{5}{|c|}{ Correlated Device } \\
Reference & 02 & 03 & 05 & 06 & DS \\
\hline 02 & 79 & 49 & 37 & 70 & 52 \\
\hline 03 & 44 & 96 & 82 & 78 & 76 \\
\hline 05 & 34 & 84 & 87 & 66 & 65 \\
\hline 06 & 61 & 76 & 60 & 97 & 79 \\
\hline DS & 45 & 72 & 59 & 78 & - \\
\hline
\end{tabular}

Table B-41. Mississippi Site 5 Right, Short Wavelengths.

\begin{tabular}{|c|c|c|c|c|c|}
\hline Correlation & \multicolumn{5}{|c|}{ Correlated Device } \\
Reference & 02 & 03 & 05 & 06 & DS \\
\hline 02 & 52 & 8 & 9 & 19 & 17 \\
\hline 03 & 11 & 86 & 41 & 24 & 40 \\
\hline 05 & 10 & 55 & 67 & 13 & 24 \\
\hline 06 & 20 & 24 & 10 & 89 & 37 \\
\hline DS & 15 & 35 & 16 & 42 & - \\
\hline
\end{tabular}


Table B-42. Mississippi Site 6 Left, IRI Filter.

\begin{tabular}{|c|c|c|c|c|c|c|}
\hline Correlation & \multicolumn{6}{|c|}{ Correlated Device } \\
Reference & 01 & 02 & 03 & 05 & 06 & DS \\
\hline 01 & 81 & 45 & 70 & 56 & 71 & 77 \\
\hline 02 & 50 & 74 & 43 & 36 & 63 & 54 \\
\hline 03 & 73 & 39 & 86 & 60 & 72 & 77 \\
\hline 05 & 60 & 34 & 61 & 46 & 57 & 60 \\
\hline 06 & 74 & 58 & 71 & 55 & $\mathbf{9 4}$ & 79 \\
\hline DS & 78 & 49 & 74 & 57 & 78 & - \\
\hline
\end{tabular}

Table B-43. Mississippi Site 6 Left, Long Wavelengths.

\begin{tabular}{|c|c|c|c|c|c|c|}
\hline $\begin{array}{c}\text { Correlation } \\
\text { Reference }\end{array}$ & \multicolumn{5}{|c|}{ Correlated Device } \\
\hline 01 & 91 & 02 & 03 & 05 & 06 & DS \\
\hline 02 & 85 & 84 & 75 & 72 & 90 & 82 \\
\hline 03 & 74 & 76 & 95 & 74 & 83 & 93 \\
\hline 05 & 72 & 66 & 74 & 69 & 78 & 77 \\
\hline 06 & 89 & 82 & 83 & 77 & 98 & 90 \\
\hline DS & 81 & 82 & 93 & 77 & 90 & - \\
\hline
\end{tabular}

Table B-44. Mississippi Site 6 Left, Medium Wavelengths.

\begin{tabular}{|c|c|c|c|c|c|c|}
\hline Correlation & \multicolumn{6}{|c|}{ Correlated Device } \\
Reference & 01 & 02 & 03 & 05 & 06 & DS \\
\hline 01 & 78 & 33 & 63 & 47 & 61 & 69 \\
\hline 02 & 39 & 68 & 33 & 28 & 57 & 45 \\
\hline 03 & 69 & 29 & 82 & 55 & 67 & 69 \\
\hline 05 & 55 & 27 & 57 & 41 & 51 & 54 \\
\hline 06 & 68 & 49 & 65 & 47 & 93 & 74 \\
\hline DS & 76 & 39 & 65 & 49 & 72 & - \\
\hline
\end{tabular}

Table B-45. Mississippi Site 6 Left, Short Wavelengths.

\begin{tabular}{|c|c|c|c|c|c|c|}
\hline $\begin{array}{c}\text { Correlation } \\
\text { Reference }\end{array}$ & \multicolumn{6}{|c|}{ Correlated Device } \\
\hline 01 & 54 & 02 & 03 & 05 & 06 & DS \\
\hline 02 & 11 & 45 & 8 & 8 & 11 & 15 \\
\hline 03 & 28 & 13 & 59 & 23 & 20 & 22 \\
\hline 05 & 22 & 12 & 23 & 16 & 15 & 16 \\
\hline 06 & 28 & 15 & 16 & 10 & 71 & 30 \\
\hline DS & 36 & 14 & 16 & 11 & 35 & - \\
\hline
\end{tabular}


Table B-46. Mississippi Site 6 Right, IRI Filter.

\begin{tabular}{|c|c|c|c|c|c|}
\hline Correlation & \multicolumn{5}{|c|}{ Correlated Device } \\
Reference & 02 & 03 & 05 & 06 & DS \\
\hline 02 & 77 & 48 & 43 & 70 & 61 \\
\hline 03 & 46 & 92 & 71 & 77 & 84 \\
\hline 05 & 42 & 70 & 60 & 67 & 67 \\
\hline 06 & 66 & 76 & 67 & $\mathbf{9 8}$ & 82 \\
\hline DS & 60 & 84 & 69 & 86 & - \\
\hline
\end{tabular}

Table B-47. Mississippi Site 6 Right, Long Wavelengths.

\begin{tabular}{|c|c|c|c|c|c|}
\hline Correlation & \multicolumn{5}{|c|}{ Correlated Device } \\
Reference & 02 & 03 & 05 & 06 & DS \\
\hline 02 & 91 & 82 & 70 & 89 & 90 \\
\hline 03 & 82 & 97 & 78 & 82 & 91 \\
\hline 05 & 69 & 77 & 77 & 75 & 75 \\
\hline 06 & 90 & 82 & 75 & 99 & 91 \\
\hline DS & 89 & 92 & 75 & 91 & - \\
\hline
\end{tabular}

Table B-48. Mississippi Site 6 Right, Medium Wavelengths.

\begin{tabular}{|c|c|c|c|c|c|}
\hline Correlation & \multicolumn{5}{|c|}{ Correlated Device } \\
Reference & 02 & 03 & 05 & 06 & DS \\
\hline 02 & 69 & 37 & 35 & 62 & 52 \\
\hline 03 & 36 & 91 & 68 & 70 & 80 \\
\hline 05 & 35 & 67 & 56 & 63 & 63 \\
\hline 06 & 56 & 68 & 59 & 97 & 77 \\
\hline DS & 50 & 77 & 64 & 83 & - \\
\hline
\end{tabular}

Table B-49. Mississippi Site 6 Right, Short Wavelengths.

\begin{tabular}{|c|c|c|c|c|c|}
\hline Correlation & \multicolumn{5}{|c|}{ Correlated Device } \\
Reference & 02 & 03 & 05 & 06 & DS \\
\hline 02 & 41 & 6 & 10 & 12 & 13 \\
\hline 03 & 11 & 71 & 31 & 11 & 39 \\
\hline 05 & 11 & 21 & 21 & 17 & 21 \\
\hline 06 & 11 & 9 & 13 & 81 & 25 \\
\hline DS & 11 & 22 & 14 & 26 & - \\
\hline
\end{tabular}


Table B-50. Mississippi Site 7 Left, IRI Filter.

\begin{tabular}{|c|c|c|c|c|c|}
\hline Correlation & \multicolumn{5}{|c|}{ Correlated Device } \\
Reference & 01 & 02 & 05 & 06 & DS \\
\hline 01 & 80 & 62 & 65 & 81 & 75 \\
\hline 02 & 64 & 82 & 53 & 75 & 64 \\
\hline 05 & 62 & 51 & 65 & 70 & 73 \\
\hline 06 & 77 & 71 & 71 & $\mathbf{9 8}$ & 85 \\
\hline DS & 71 & 60 & 73 & 84 & - \\
\hline
\end{tabular}

Table B-51. Mississippi Site 7 Left, Long Wavelengths.

\begin{tabular}{|c|c|c|c|c|c|}
\hline Correlation & \multicolumn{5}{|c|}{ Correlated Device } \\
Reference & 01 & 02 & 05 & 06 & DS \\
\hline 01 & 96 & 90 & 77 & 95 & 85 \\
\hline 02 & 90 & 96 & 73 & 91 & 85 \\
\hline 05 & 76 & 73 & 89 & 79 & 82 \\
\hline 06 & 95 & 91 & 79 & 99 & 88 \\
\hline DS & 85 & 84 & 82 & 88 & - \\
\hline
\end{tabular}

Table B-52. Mississippi Site 7 Left, Medium Wavelengths.

\begin{tabular}{|c|c|c|c|c|c|}
\hline Correlation & \multicolumn{5}{|c|}{ Correlated Device } \\
Reference & 01 & 02 & 05 & 06 & DS \\
\hline 01 & 74 & 48 & 59 & 76 & 72 \\
\hline 02 & 52 & 72 & 44 & 68 & 54 \\
\hline 05 & 56 & 40 & 54 & 64 & 66 \\
\hline 06 & 71 & 61 & 65 & 97 & 83 \\
\hline DS & 66 & 47 & 63 & 81 & - \\
\hline
\end{tabular}

Table B-53. Mississippi Site 7 Left, Short Wavelengths.

\begin{tabular}{|c|c|c|c|c|c|}
\hline Correlation & \multicolumn{5}{|c|}{ Correlated Device } \\
Reference & 01 & 02 & 05 & 06 & DS \\
\hline 01 & 37 & 6 & 7 & 16 & 21 \\
\hline 02 & 11 & 31 & 9 & 14 & 9 \\
\hline 05 & 22 & 9 & 14 & 18 & 21 \\
\hline 06 & 28 & 11 & 9 & 70 & 40 \\
\hline DS & 29 & 6 & 10 & 36 & - \\
\hline
\end{tabular}


Table B-54. Mississippi Site 7 Right, IRI Filter.

\begin{tabular}{|c|c|c|c|c|}
\hline Correlation & \multicolumn{4}{|c|}{ Correlated Device } \\
Reference & 02 & 05 & 06 & DS \\
\hline 02 & 79 & 49 & 71 & 65 \\
\hline 05 & 47 & 59 & 70 & 68 \\
\hline 06 & 67 & 71 & $\mathbf{9 8}$ & 90 \\
\hline DS & 61 & 69 & 89 & - \\
\hline
\end{tabular}

Table B-55. Mississippi Site 7 Right, Long Wavelengths.

\begin{tabular}{|c|c|c|c|c|}
\hline Correlation & \multicolumn{4}{|c|}{ Correlated Device } \\
Reference & 02 & 05 & 06 & DS \\
\hline 02 & 96 & 70 & 89 & 85 \\
\hline 05 & 70 & 79 & 77 & 76 \\
\hline 06 & 89 & 77 & 99 & 90 \\
\hline DS & 84 & 77 & 90 & - \\
\hline
\end{tabular}

Table B-56. Mississippi Site 7 Right, Medium Wavelengths.

\begin{tabular}{|c|c|c|c|c|}
\hline Correlation & \multicolumn{4}{|c|}{ Correlated Device } \\
Reference & 02 & 05 & 06 & DS \\
\hline 02 & 67 & 42 & 63 & 56 \\
\hline 05 & 38 & 51 & 65 & 63 \\
\hline 06 & 56 & 64 & 98 & 88 \\
\hline DS & 49 & 62 & 89 & - \\
\hline
\end{tabular}

Table B-57. Mississippi Site 7 Right, Short Wavelengths.

\begin{tabular}{|c|c|c|c|c|}
\hline Correlation & \multicolumn{4}{|c|}{ Correlated Device } \\
Reference & 02 & 05 & 06 & DS \\
\hline 02 & 28 & 10 & 10 & 9 \\
\hline 05 & 9 & 17 & 20 & 25 \\
\hline 06 & 9 & 13 & 77 & 42 \\
\hline DS & 7 & 13 & 36 & - \\
\hline
\end{tabular}


Table B-58. Mississippi Site 8 Left, IRI Filter.

\begin{tabular}{|c|c|c|c|c|c|c|}
\hline Correlation & \multicolumn{6}{|c|}{ Correlated Device } \\
Reference & 01 & 02 & 03 & 05 & 06 & DS \\
\hline 01 & 57 & 33 & 60 & 47 & 57 & 57 \\
\hline 02 & 35 & 55 & 39 & 34 & 52 & 46 \\
\hline 03 & 56 & 36 & 87 & 60 & 76 & 74 \\
\hline 05 & 46 & 31 & 63 & 50 & 62 & 61 \\
\hline 06 & 53 & 47 & 77 & 58 & 93 & 78 \\
\hline DS & 54 & 41 & 80 & 61 & 84 & - \\
\hline
\end{tabular}

Table B-59. Mississippi Site 8 Left, Long Wavelengths.

\begin{tabular}{|c|c|c|c|c|c|c|}
\hline Correlation & \multicolumn{5}{|c|}{ Correlated Device } \\
Reference & 01 & 02 & 03 & 05 & 06 & DS \\
\hline 01 & 87 & 70 & 77 & 71 & 89 & 80 \\
\hline 02 & 71 & 86 & 74 & 57 & 76 & 77 \\
\hline 03 & 77 & 73 & 98 & 70 & 83 & 95 \\
\hline 05 & 71 & 56 & 70 & 66 & 77 & 71 \\
\hline 06 & 89 & 74 & 83 & 77 & 98 & 88 \\
\hline DS & 80 & 76 & 95 & 71 & 87 & - \\
\hline
\end{tabular}

Table B-60. Mississippi Site 8 Left, Medium Wavelengths.

\begin{tabular}{|c|c|c|c|c|c|c|}
\hline Correlation & \multicolumn{6}{|c|}{ Correlated Device } \\
Reference & 01 & 02 & 03 & 05 & 06 & DS \\
\hline 01 & 53 & 28 & 61 & 44 & 55 & 55 \\
\hline 02 & 32 & 48 & 36 & 31 & 49 & 41 \\
\hline 03 & 56 & 31 & 84 & 57 & 71 & 69 \\
\hline 05 & 44 & 27 & 62 & 47 & 58 & 58 \\
\hline 06 & 51 & 41 & 72 & 52 & 91 & 72 \\
\hline DS & 53 & 35 & 78 & 57 & 81 & - \\
\hline
\end{tabular}

Table B-61. Mississippi Site 8 Left, Short Wavelengths.

\begin{tabular}{|c|c|c|c|c|c|c|}
\hline $\begin{array}{c}\text { Correlation } \\
\text { Reference }\end{array}$ & \multicolumn{6}{|c|}{ Correlated Device } \\
\hline 01 & 30 & 5 & 16 & 8 & 8 & 11 \\
\hline 02 & 9 & 19 & 8 & 7 & 9 & 8 \\
\hline 03 & 28 & 6 & 55 & 22 & 14 & 28 \\
\hline 05 & 20 & 6 & 26 & 17 & 15 & 18 \\
\hline 06 & 20 & 8 & 15 & 11 & 53 & 28 \\
\hline DS & 24 & 7 & 38 & 18 & 35 & - \\
\hline
\end{tabular}


Table B-62. Mississippi Site 8 Right, IRI Filter.

\begin{tabular}{|c|c|c|c|c|c|}
\hline Correlation & \multicolumn{5}{|c|}{ Correlated Device } \\
Reference & 02 & 03 & 05 & 06 & DS \\
\hline 02 & 57 & 43 & 42 & 55 & 52 \\
\hline 03 & 40 & 93 & 62 & 80 & 75 \\
\hline 05 & 40 & 67 & 57 & 66 & 66 \\
\hline 06 & 51 & 80 & 61 & $\mathbf{9 7}$ & 74 \\
\hline DS & 47 & 81 & 63 & 80 & - \\
\hline
\end{tabular}

Table B-63. Mississippi Site 8 Right, Long Wavelengths.

\begin{tabular}{|c|c|c|c|c|c|}
\hline Correlation & \multicolumn{5}{|c|}{ Correlated Device } \\
Reference & 02 & 03 & 05 & 06 & DS \\
\hline 02 & 90 & 80 & 67 & 80 & 83 \\
\hline 03 & 80 & 98 & 74 & 83 & 95 \\
\hline 05 & 66 & 74 & 78 & 83 & 75 \\
\hline 06 & 79 & 83 & 84 & 99 & 85 \\
\hline DS & 82 & 95 & 75 & 87 & - \\
\hline
\end{tabular}

Table B-64. Mississippi Site 8 Right, Medium Wavelengths.

\begin{tabular}{|c|c|c|c|c|c|}
\hline Correlation & \multicolumn{5}{|c|}{ Correlated Device } \\
Reference & 02 & 03 & 05 & 06 & DS \\
\hline 02 & 51 & 36 & 35 & 49 & 45 \\
\hline 03 & 31 & 91 & 59 & 76 & 69 \\
\hline 05 & 33 & 66 & 54 & 62 & 61 \\
\hline 06 & 42 & 75 & 56 & 96 & 69 \\
\hline DS & 38 & 79 & 58 & 78 & - \\
\hline
\end{tabular}

Table B-65. Mississippi Site 8 Right, Short Wavelengths.

\begin{tabular}{|c|c|c|c|c|c|}
\hline Correlation & \multicolumn{5}{|c|}{ Correlated Device } \\
Reference & 02 & 03 & 05 & 06 & DS \\
\hline 02 & 27 & 9 & 9 & 13 & 11 \\
\hline 03 & 6 & 70 & 24 & 20 & 31 \\
\hline 05 & 8 & 32 & 19 & 21 & 22 \\
\hline 06 & 9 & 21 & 14 & 79 & 32 \\
\hline DS & 7 & 37 & 17 & 44 & - \\
\hline
\end{tabular}


Table B-66. Nevada Site 1 Left, IRI Filter.

\begin{tabular}{|c|c|c|c|c|c|c|c|}
\hline $\begin{array}{c}\text { Correlation } \\
\text { Reference }\end{array}$ & \multicolumn{6}{|c|}{ Correlated Device } \\
\hline 03 & 68 & 42 & 70 & 61 & 60 & 60 & 60 \\
\hline 04 & 47 & 51 & 47 & 42 & 38 & 35 & 35 \\
\hline 06 & 63 & 42 & 78 & 77 & 67 & 66 & 66 \\
\hline 07 & 55 & 39 & 73 & 92 & 66 & 57 & 57 \\
\hline 08 & 55 & 36 & 66 & 68 & $\mathbf{9 4}$ & 75 & 75 \\
\hline 09 & 54 & 32 & 62 & 58 & 74 & 91 & 93 \\
\hline DS & 54 & 33 & 63 & 58 & 73 & 93 & - \\
\hline
\end{tabular}

Table B-67. Nevada Site 1 Left, Long Wavelengths.

\begin{tabular}{|c|c|c|c|c|c|c|c|}
\hline $\begin{array}{c}\text { Correlation } \\
\text { Reference }\end{array}$ & \multicolumn{6}{|c|}{ Correlated Device } \\
\hline 03 & 92 & 31 & 77 & 80 & 73 & 77 & 81 \\
\hline 04 & 31 & 57 & 28 & 25 & 27 & 27 & 29 \\
\hline 06 & 77 & 28 & 93 & 71 & 88 & 92 & 92 \\
\hline 07 & 80 & 24 & 71 & 97 & 75 & 75 & 76 \\
\hline 08 & 73 & 27 & 88 & 75 & 96 & 92 & 90 \\
\hline 09 & 76 & 27 & 91 & 74 & 92 & 97 & 96 \\
\hline DS & 80 & 29 & 92 & 75 & 90 & 96 & - \\
\hline
\end{tabular}

Table B-68. Nevada Site 1 Left, Medium Wavelengths.

\begin{tabular}{|c|c|c|c|c|c|c|c|}
\hline $\begin{array}{c}\text { Correlation } \\
\text { Reference }\end{array}$ & \multicolumn{6}{|c|}{ Correlated Device } \\
\hline 03 & 60 & 39 & 65 & 55 & 56 & 55 & 55 \\
\hline 04 & 48 & 48 & 46 & 43 & 38 & 34 & 34 \\
\hline 06 & 54 & 38 & 76 & 76 & 64 & 61 & 62 \\
\hline 07 & 47 & 37 & 71 & 90 & 62 & 52 & 52 \\
\hline 08 & 49 & 33 & 64 & 65 & 93 & 71 & 69 \\
\hline 09 & 46 & 29 & 58 & 54 & 69 & 88 & 91 \\
\hline DS & 46 & 30 & 59 & 53 & 67 & 91 & - \\
\hline
\end{tabular}

Table B-69. Nevada Site 1 Left, Short Wavelengths.

\begin{tabular}{|c|c|c|c|c|c|c|c|}
\hline $\begin{array}{c}\text { Correlation } \\
\text { Reference }\end{array}$ & \multicolumn{7}{|c|}{ Correlated Device } \\
\hline 03 & 29 & 14 & 22 & 20 & 23 & 18 & 16 \\
\hline 04 & 20 & 27 & 16 & 20 & 13 & 13 & 13 \\
\hline 06 & 14 & 11 & 47 & 22 & 21 & 12 & 19 \\
\hline 07 & 16 & 16 & 35 & 64 & 27 & 17 & 23 \\
\hline 08 & 17 & 11 & 31 & 23 & 77 & 20 & 26 \\
\hline 09 & 12 & 9 & 27 & 21 & 25 & 56 & 55 \\
\hline DS & 12 & 9 & 28 & 19 & 25 & 55 & - \\
\hline
\end{tabular}


Table B-70. Nevada Site 1 Right, IRI Filter.

\begin{tabular}{|c|c|c|c|c|c|c|c|}
\hline $\begin{array}{c}\text { Correlation } \\
\text { Reference }\end{array}$ & \multicolumn{6}{|c|}{ Correlated Device } \\
\hline 03 & 77 & 50 & 77 & 74 & 76 & 71 & 74 \\
\hline 04 & 55 & 56 & 52 & 50 & 47 & 43 & 44 \\
\hline 06 & 71 & 47 & 81 & 82 & 82 & 77 & 79 \\
\hline 07 & 69 & 46 & 77 & 93 & 82 & 74 & 76 \\
\hline 08 & 71 & 44 & 80 & 83 & 92 & 85 & 88 \\
\hline 09 & 65 & 40 & 72 & 74 & 84 & 90 & 93 \\
\hline DS & 68 & 41 & 76 & 76 & 87 & 93 & - \\
\hline
\end{tabular}

Table B-71. Nevada Site 1 Right, Long Wavelengths.

\begin{tabular}{|c|c|c|c|c|c|c|c|}
\hline $\begin{array}{c}\text { Correlation } \\
\text { Reference }\end{array}$ & \multicolumn{6}{|c|}{ Correlated Device } \\
\hline 03 & 95 & 34 & 85 & 83 & 81 & 83 & 84 \\
\hline 04 & 34 & 70 & 29 & 29 & 29 & 30 & 30 \\
\hline 06 & 84 & 29 & 95 & 73 & 87 & 91 & 92 \\
\hline 07 & 82 & 28 & 72 & 98 & 85 & 81 & 82 \\
\hline 08 & 81 & 29 & 87 & 85 & 97 & 95 & 96 \\
\hline 09 & 83 & 30 & 91 & 81 & 95 & 97 & 98 \\
\hline DS & 83 & 30 & 91 & 82 & 96 & 98 & - \\
\hline
\end{tabular}

Table B-72. Nevada Site 1 Right, Medium Wavelengths.

\begin{tabular}{|c|c|c|c|c|c|c|c|}
\hline $\begin{array}{c}\text { Correlation } \\
\text { Reference }\end{array}$ & 03 & 04 & 06 & 07 & 08 & 09 & DS \\
\hline 03 & 70 & 46 & 72 & 70 & 73 & 66 & 69 \\
\hline 04 & 55 & 52 & 51 & 52 & 47 & 42 & 43 \\
\hline 06 & 62 & 44 & 75 & 81 & 78 & 72 & 74 \\
\hline 07 & 62 & 46 & 77 & 92 & 80 & 70 & 73 \\
\hline 08 & 65 & 41 & 76 & 81 & 89 & 81 & 84 \\
\hline 09 & 57 & 36 & 67 & 70 & 80 & 87 & 91 \\
\hline DS & 60 & 37 & 70 & 71 & 82 & 91 & - \\
\hline
\end{tabular}

Table B-73. Nevada Site 1 Right, Short Wavelengths.

\begin{tabular}{|c|c|c|c|c|c|c|c|}
\hline $\begin{array}{c}\text { Correlation } \\
\text { Reference }\end{array}$ & \multicolumn{7}{|c|}{ Correlated Device } \\
\hline 03 & 36 & 17 & 29 & 27 & 36 & 24 & 23 \\
\hline 04 & 22 & 30 & 19 & 25 & 18 & 13 & 12 \\
\hline 06 & 19 & 14 & 41 & 26 & 32 & 18 & 27 \\
\hline 07 & 21 & 20 & 39 & 68 & 40 & 23 & 25 \\
\hline 08 & 27 & 15 & 40 & 31 & 70 & 33 & 45 \\
\hline 09 & 18 & 10 & 31 & 22 & 42 & 69 & 62 \\
\hline DS & 19 & 10 & 33 & 20 & 39 & 61 & - \\
\hline
\end{tabular}


Table B-74. Nevada Site 2 Left, IRI Filter.

\begin{tabular}{|c|c|c|c|c|c|c|c|}
\hline $\begin{array}{c}\text { Correlation } \\
\text { Reference }\end{array}$ & \multicolumn{7}{|c|}{ Correlated Device } \\
\hline 03 & 37 & 23 & 33 & 35 & 38 & 32 & 31 \\
\hline 04 & 26 & 21 & 20 & 21 & 23 & 19 & 19 \\
\hline 06 & 30 & 18 & 48 & 50 & 55 & 48 & 47 \\
\hline 07 & 33 & 19 & 47 & 91 & 67 & 64 & 64 \\
\hline 08 & 35 & 21 & 51 & 69 & 89 & 62 & 61 \\
\hline 09 & 30 & 17 & 44 & 64 & 61 & 80 & 83 \\
\hline DS & 29 & 18 & 44 & 64 & 60 & 83 & - \\
\hline
\end{tabular}

Table B-75. Nevada Site 2 Left, Long Wavelengths.

\begin{tabular}{|c|c|c|c|c|c|c|c|}
\hline $\begin{array}{c}\text { Correlation } \\
\text { Reference }\end{array}$ & \multicolumn{7}{|c|}{ Correlated Device } \\
\hline 03 & 81 & 53 & 55 & 77 & 63 & 64 & 65 \\
\hline 04 & 54 & 69 & 36 & 51 & 37 & 39 & 41 \\
\hline 06 & 57 & 37 & 84 & 57 & 83 & 85 & 81 \\
\hline 07 & 76 & 51 & 53 & 98 & 60 & 62 & 63 \\
\hline 08 & 65 & 39 & 83 & 64 & 96 & 94 & 92 \\
\hline 09 & 65 & 41 & 85 & 66 & 93 & 95 & 94 \\
\hline DS & 68 & 44 & 82 & 69 & 92 & 95 & - \\
\hline
\end{tabular}

Table B-76. Nevada Site 2 Left, Medium Wavelengths.

\begin{tabular}{|c|c|c|c|c|c|c|c|}
\hline Correlation & \multicolumn{6}{|c|}{ Correlated Device } \\
Reference & 03 & 04 & 06 & 07 & 08 & 09 & DS \\
\hline 03 & 25 & 15 & 25 & 24 & 29 & 23 & 21 \\
\hline 04 & 18 & 14 & 14 & 14 & 17 & 13 & 13 \\
\hline 06 & 21 & 12 & 36 & 41 & 44 & 35 & 34 \\
\hline 07 & 21 & 12 & 37 & 87 & 56 & 44 & 47 \\
\hline 08 & 25 & 14 & 40 & 58 & 84 & 48 & 47 \\
\hline 09 & 19 & 11 & 31 & 47 & 47 & 63 & 69 \\
\hline DS & 18 & 11 & 31 & 50 & 45 & 69 & - \\
\hline
\end{tabular}

Table B-77. Nevada Site 2 Left, Short Wavelengths.

\begin{tabular}{|c|c|c|c|c|c|c|c|}
\hline $\begin{array}{c}\text { Correlation } \\
\text { Reference }\end{array}$ & \multicolumn{7}{|c|}{ Correlated Device } \\
\hline 03 & 11 & 6 & 9 & 8 & 12 & 7 & 8 \\
\hline 04 & 11 & 9 & 5 & 5 & 7 & 6 & 5 \\
\hline 06 & 5 & 3 & 18 & 16 & 13 & 3 & 4 \\
\hline 07 & 6 & 4 & 18 & 52 & 15 & 4 & 6 \\
\hline 08 & 8 & 5 & 17 & 17 & 53 & 4 & 7 \\
\hline 09 & 6 & 4 & 8 & 5 & 6 & 19 & 19 \\
\hline DS & 6 & 4 & 9 & 9 & 10 & 19 & - \\
\hline
\end{tabular}


Table B-78. Nevada Site 2 Right, IRI Filter.

\begin{tabular}{|c|c|c|c|c|c|c|c|}
\hline $\begin{array}{c}\text { Correlation } \\
\text { Reference }\end{array}$ & \multicolumn{6}{|c|}{ Correlated Device } \\
\hline 03 & 49 & 32 & 41 & 39 & 42 & 38 & 37 \\
\hline 04 & 36 & 30 & 30 & 29 & 29 & 23 & 23 \\
\hline 06 & 39 & 27 & 45 & 50 & 53 & 42 & 41 \\
\hline 07 & 36 & 26 & 47 & 93 & 79 & 61 & 60 \\
\hline 08 & 39 & 26 & 49 & 80 & 91 & 79 & 77 \\
\hline 09 & 35 & 21 & 39 & 61 & 77 & $\mathbf{9 5}$ & $\mathbf{9 5}$ \\
\hline DS & 34 & 21 & 38 & 59 & 75 & $\mathbf{9 5}$ & - \\
\hline
\end{tabular}

Table B-79. Nevada Site 2 Right, Long Wavelengths.

\begin{tabular}{|c|c|c|c|c|c|c|c|}
\hline $\begin{array}{c}\text { Correlation } \\
\text { Reference }\end{array}$ & \multicolumn{8}{|c|}{ Correlated Device } \\
\hline 03 & 84 & 64 & 61 & 76 & 65 & 65 & 62 \\
\hline 04 & 64 & 77 & 46 & 56 & 46 & 47 & 45 \\
\hline 06 & 61 & 46 & 85 & 59 & 86 & 85 & 86 \\
\hline 07 & 75 & 56 & 59 & 97 & 66 & 64 & 64 \\
\hline 08 & 65 & 46 & 86 & 67 & 98 & 96 & 96 \\
\hline 09 & 65 & 47 & 85 & 65 & 96 & 96 & 96 \\
\hline DS & 63 & 45 & 86 & 65 & 96 & 97 & - \\
\hline
\end{tabular}

Table B-80. Nevada Site 2 Right, Medium Wavelengths.

\begin{tabular}{|c|c|c|c|c|c|c|c|}
\hline $\begin{array}{c}\text { Correlation } \\
\text { Reference }\end{array}$ & \multicolumn{7}{|c|}{ Correlated Device } \\
\hline 03 & 40 & 23 & 34 & 30 & 37 & 30 & 28 \\
\hline 04 & 28 & 24 & 25 & 23 & 24 & 17 & 16 \\
\hline 06 & 31 & 21 & 38 & 45 & 47 & 33 & 32 \\
\hline 07 & 26 & 19 & 40 & 90 & 72 & 50 & 49 \\
\hline 08 & 32 & 21 & 42 & 74 & 86 & 68 & 66 \\
\hline 09 & 26 & 15 & 29 & 49 & 66 & 92 & 92 \\
\hline DS & 25 & 14 & 28 & 46 & 63 & 92 & - \\
\hline
\end{tabular}

Table B-81. Nevada Site 2 Right, Short Wavelengths.

\begin{tabular}{|c|c|c|c|c|c|c|c|}
\hline $\begin{array}{c}\text { Correlation } \\
\text { Reference }\end{array}$ & \multicolumn{7}{|c|}{ Correlated Device } \\
\hline 03 & 20 & 8 & 13 & 10 & 16 & 10 & 8 \\
\hline 04 & 12 & 12 & 10 & 9 & 9 & 5 & 4 \\
\hline 06 & 8 & 6 & 16 & 18 & 17 & 9 & 10 \\
\hline 07 & 6 & 7 & 17 & 65 & 24 & 17 & 17 \\
\hline 08 & 11 & 7 & 16 & 23 & 51 & 14 & 14 \\
\hline 09 & 6 & 3 & 8 & 18 & 14 & 75 & 60 \\
\hline DS & 5 & 3 & 7 & 14 & 11 & 60 & - \\
\hline
\end{tabular}


Table B-82. Nevada Site 3 Left, IRI Filter.

\begin{tabular}{|c|c|c|c|c|c|c|c|}
\hline $\begin{array}{c}\text { Correlation } \\
\text { Reference }\end{array}$ & \multicolumn{6}{|c|}{ Correlated Device } \\
\hline 03 & 66 & 54 & 33 & 53 & 51 & 62 & 64 \\
\hline 04 & 60 & 65 & 31 & 42 & 40 & 59 & 61 \\
\hline 06 & 35 & 30 & 36 & 24 & 22 & 35 & 37 \\
\hline 07 & 48 & 38 & 23 & 77 & 72 & 37 & 37 \\
\hline 08 & 46 & 36 & 20 & 72 & 81 & 40 & 40 \\
\hline 09 & 64 & 56 & 34 & 39 & 41 & 85 & 90 \\
\hline DS & 67 & 57 & 36 & 38 & 41 & 89 & - \\
\hline
\end{tabular}

Table B-83. Nevada Site 3 Left, Long Wavelengths.

\begin{tabular}{|c|c|c|c|c|c|c|c|}
\hline $\begin{array}{c}\text { Correlation } \\
\text { Reference }\end{array}$ & \multicolumn{6}{|c|}{ Correlated Device } \\
\hline 03 & 86 & 30 & 70 & 70 & 61 & 77 & 79 \\
\hline 04 & 31 & 41 & 27 & 21 & 17 & 27 & 27 \\
\hline 06 & 70 & 27 & 76 & 47 & 59 & 80 & 82 \\
\hline 07 & 70 & 21 & 47 & 90 & 64 & 55 & 55 \\
\hline 08 & 60 & 17 & 59 & 64 & 93 & 71 & 71 \\
\hline 09 & 77 & 26 & 80 & 55 & 72 & 92 & 95 \\
\hline DS & 79 & 27 & 81 & 55 & 71 & 95 & - \\
\hline
\end{tabular}

Table B-84. Nevada Site 3 Left, Medium Wavelengths.

\begin{tabular}{|c|c|c|c|c|c|c|c|}
\hline Correlation & \multicolumn{6}{|c|}{ Correlated Device } \\
Reference & 03 & 04 & 06 & 07 & 08 & 09 & DS \\
\hline 03 & 61 & 54 & 32 & 50 & 49 & 58 & 61 \\
\hline 04 & 62 & 64 & 35 & 44 & 42 & 58 & 61 \\
\hline 06 & 34 & 32 & 37 & 24 & 22 & 34 & 37 \\
\hline 07 & 43 & 37 & 22 & 75 & 70 & 33 & 33 \\
\hline 08 & 42 & 36 & 20 & 70 & 80 & 37 & 37 \\
\hline 09 & 59 & 53 & 35 & 35 & 39 & 84 & 89 \\
\hline DS & 62 & 55 & 37 & 35 & 39 & 89 & - \\
\hline
\end{tabular}

Table B-85. Nevada Site 3 Left, Short Wavelengths.

\begin{tabular}{|c|c|c|c|c|c|c|c|}
\hline Correlation & \multicolumn{6}{|c|}{ Correlated Device } \\
Reference & 03 & 04 & 06 & 07 & 08 & 09 & DS \\
\hline 03 & 36 & 25 & 20 & 27 & 36 & 21 & 22 \\
\hline 04 & 32 & 37 & 18 & 25 & 28 & 17 & 16 \\
\hline 06 & 13 & 12 & 25 & 21 & 26 & 10 & 12 \\
\hline 07 & 19 & 18 & 19 & 75 & 49 & 11 & 13 \\
\hline 08 & 24 & 20 & 20 & 45 & 78 & 15 & 17 \\
\hline 09 & 19 & 15 & 16 & 13 & 18 & 73 & 71 \\
\hline DS & 19 & 15 & 16 & 15 & 20 & 71 & - \\
\hline
\end{tabular}


Table B-86. Nevada Site 3 Right, IRI Filter.

\begin{tabular}{|c|c|c|c|c|c|c|c|}
\hline $\begin{array}{c}\text { Correlation } \\
\text { Reference }\end{array}$ & \multicolumn{7}{|c|}{ Correlated Device } \\
\hline 03 & 55 & 33 & 36 & 49 & 41 & 50 & 46 \\
\hline 04 & 37 & 40 & 22 & 31 & 25 & 29 & 26 \\
\hline 06 & 33 & 20 & 34 & 38 & 34 & 37 & 35 \\
\hline 07 & 45 & 28 & 36 & 90 & 67 & 50 & 49 \\
\hline 08 & 38 & 23 & 31 & 65 & 91 & 63 & 66 \\
\hline 09 & 46 & 26 & 35 & 52 & 64 & $\mathbf{9 4}$ & 92 \\
\hline DS & 43 & 24 & 33 & 51 & 68 & 92 & - \\
\hline
\end{tabular}

Table B-87. Nevada Site 3 Right, Long Wavelengths.

\begin{tabular}{|c|c|c|c|c|c|c|c|}
\hline $\begin{array}{c}\text { Correlation } \\
\text { Reference }\end{array}$ & \multicolumn{6}{|c|}{ Correlated Device } \\
\hline 03 & 90 & 25 & 71 & 78 & 68 & 74 & 69 \\
\hline 04 & 26 & 74 & 20 & 20 & 14 & 16 & 14 \\
\hline 06 & 69 & 15 & 75 & 61 & 76 & 80 & 79 \\
\hline 07 & 78 & 19 & 62 & 96 & 71 & 71 & 69 \\
\hline 08 & 67 & 14 & 75 & 71 & 98 & 92 & 95 \\
\hline 09 & 73 & 16 & 80 & 71 & 92 & 97 & 96 \\
\hline DS & 69 & 14 & 78 & 70 & 95 & 96 & - \\
\hline
\end{tabular}

Table B-88. Nevada Site 3 Right, Medium Wavelengths.

\begin{tabular}{|c|c|c|c|c|c|c|c|}
\hline $\begin{array}{c}\text { Correlation } \\
\text { Reference }\end{array}$ & 03 & 04 & 06 & 07 & 08 & 09 & DS \\
\hline 03 & 39 & 25 & 21 & 39 & 31 & 38 & 33 \\
\hline 04 & 31 & 28 & 16 & 28 & 22 & 23 & 21 \\
\hline 06 & 17 & 12 & 23 & 26 & 23 & 23 & 22 \\
\hline 07 & 32 & 23 & 23 & 86 & 59 & 41 & 39 \\
\hline 08 & 26 & 18 & 21 & 57 & 88 & 51 & 56 \\
\hline 09 & 31 & 19 & 21 & 43 & 54 & 93 & 90 \\
\hline DS & 28 & 17 & 20 & 41 & 58 & 90 & - \\
\hline
\end{tabular}

Table B-89. Nevada Site 3 Right, Short Wavelengths.

\begin{tabular}{|c|c|c|c|c|c|c|c|}
\hline $\begin{array}{c}\text { Correlation } \\
\text { Reference }\end{array}$ & 03 & 04 & 06 & 07 & 08 & 09 & DS \\
\hline 03 & 20 & 10 & 11 & 16 & 16 & 13 & 9 \\
\hline 04 & 14 & 16 & 9 & 14 & 11 & 9 & 7 \\
\hline 06 & 6 & 5 & 17 & 15 & 19 & 8 & 9 \\
\hline 07 & 10 & 9 & 18 & 76 & 21 & 22 & 20 \\
\hline 08 & 10 & 7 & 16 & 17 & 76 & 12 & 18 \\
\hline 09 & 8 & 6 & 10 & 25 & 15 & 84 & 69 \\
\hline DS & 6 & 5 & 8 & 16 & 19 & 69 & - \\
\hline
\end{tabular}


Table B-90. Nevada Site 4 Left, IRI Filter.

\begin{tabular}{|c|c|c|c|c|c|c|c|}
\hline Correlation & \multicolumn{6}{|c|}{ Correlated Device } \\
Reference & 03 & 04 & 06 & 07 & 08 & 09 & DS \\
\hline 03 & 60 & 64 & 49 & 54 & 69 & 60 & 58 \\
\hline 04 & 59 & 80 & 63 & 69 & 72 & 66 & 68 \\
\hline 06 & 50 & 66 & 60 & 64 & 59 & 59 & 65 \\
\hline 07 & 50 & 64 & 55 & 87 & 58 & 46 & 50 \\
\hline 08 & 67 & 74 & 55 & 60 & 86 & 72 & 67 \\
\hline 09 & 57 & 67 & 56 & 47 & 71 & 84 & 85 \\
\hline DS & 54 & 65 & 59 & 51 & 65 & 85 & - \\
\hline
\end{tabular}

Table B-91. Nevada Site 4 Left, Long Wavelengths.

\begin{tabular}{|c|c|c|c|c|c|c|c|}
\hline $\begin{array}{c}\text { Correlation } \\
\text { Reference }\end{array}$ & \multicolumn{6}{|c|}{ Correlated Device } \\
\hline 03 & 90 & 81 & 70 & 73 & 80 & 80 & 80 \\
\hline 04 & 81 & 84 & 67 & 67 & 77 & 78 & 78 \\
\hline 06 & 81 & 78 & 79 & 73 & 87 & 90 & 90 \\
\hline 07 & 73 & 67 & 62 & 95 & 81 & 78 & 78 \\
\hline 08 & 80 & 77 & 75 & 82 & 98 & 94 & 93 \\
\hline 09 & 79 & 77 & 78 & 79 & 94 & 96 & 97 \\
\hline DS & 79 & 77 & 78 & 79 & 93 & 97 & - \\
\hline
\end{tabular}

Table B-92. Nevada Site 4 Left, Medium Wavelengths.

\begin{tabular}{|c|c|c|c|c|c|c|c|}
\hline $\begin{array}{c}\text { Correlation } \\
\text { Reference }\end{array}$ & \multicolumn{6}{|c|}{ Correlated Device } \\
\hline 03 & 47 & 56 & 37 & 46 & 62 & 50 & 49 \\
\hline 04 & 49 & 73 & 54 & 66 & 66 & 57 & 61 \\
\hline 06 & 37 & 54 & 48 & 62 & 49 & 45 & 53 \\
\hline 07 & 40 & 58 & 50 & 83 & 54 & 38 & 44 \\
\hline 08 & 59 & 69 & 44 & 56 & 85 & 66 & 59 \\
\hline 09 & 46 & 58 & 42 & 39 & 64 & 76 & 77 \\
\hline DS & 43 & 55 & 47 & 45 & 58 & 77 & - \\
\hline
\end{tabular}

Table B-93. Nevada Site 4 Left, Short Wavelengths.

\begin{tabular}{|c|c|c|c|c|c|c|c|}
\hline $\begin{array}{c}\text { Correlation } \\
\text { Reference }\end{array}$ & 03 & 04 & 06 & 07 & 08 & 09 & DS \\
\hline 03 & 20 & 23 & 12 & 11 & 32 & 11 & 14 \\
\hline 04 & 16 & 39 & 25 & 23 & 35 & 10 & 13 \\
\hline 06 & 9 & 17 & 31 & 13 & 20 & 5 & 8 \\
\hline 07 & 10 & 23 & 19 & 52 & 21 & 8 & 11 \\
\hline 08 & 24 & 32 & 24 & 18 & 66 & 13 & 23 \\
\hline 09 & 12 & 15 & 11 & 10 & 18 & 34 & 32 \\
\hline DS & 11 & 13 & 12 & 9 & 21 & 32 & - \\
\hline
\end{tabular}


Table B-94. Nevada Site 4 Right, IRI Filter.

\begin{tabular}{|c|c|c|c|c|c|c|c|}
\hline $\begin{array}{c}\text { Correlation } \\
\text { Reference }\end{array}$ & \multicolumn{6}{|c|}{ Correlated Device } \\
\hline 03 & 59 & 66 & 54 & 54 & 65 & 62 & 62 \\
\hline 04 & 61 & 77 & 64 & 69 & 75 & 66 & 68 \\
\hline 06 & 56 & 69 & 62 & 66 & 70 & 62 & 66 \\
\hline 07 & 50 & 64 & 56 & 88 & 61 & 50 & 56 \\
\hline 08 & 61 & 74 & 62 & 63 & 84 & 77 & 80 \\
\hline 09 & 58 & 65 & 55 & 51 & 77 & 84 & 87 \\
\hline DS & 58 & 64 & 56 & 58 & 79 & 87 & - \\
\hline
\end{tabular}

Table B-95. Nevada Site 4 Right, Long Wavelengths.

\begin{tabular}{|c|c|c|c|c|c|c|c|}
\hline $\begin{array}{c}\text { Correlation } \\
\text { Reference }\end{array}$ & 03 & 04 & 06 & 07 & 08 & 09 & DS \\
\hline 03 & 90 & 83 & 73 & 75 & 79 & 82 & 82 \\
\hline 04 & 82 & 87 & 70 & 72 & 79 & 81 & 81 \\
\hline 06 & 84 & 81 & 78 & 74 & 87 & 89 & 88 \\
\hline 07 & 74 & 72 & 63 & 94 & 85 & 82 & 83 \\
\hline 08 & 79 & 79 & 74 & 85 & 98 & 95 & 96 \\
\hline 09 & 81 & 81 & 76 & 82 & 96 & 98 & 98 \\
\hline DS & 81 & 80 & 75 & 83 & 96 & 98 & - \\
\hline
\end{tabular}

Table B-96. Nevada Site 4 Right, Medium Wavelengths.

\begin{tabular}{|c|c|c|c|c|c|c|c|}
\hline $\begin{array}{c}\text { Correlation } \\
\text { Reference }\end{array}$ & 03 & 04 & 06 & 07 & 08 & 09 & DS \\
\hline 03 & 46 & 56 & 43 & 46 & 58 & 51 & 53 \\
\hline 04 & 51 & 71 & 56 & 66 & 69 & 56 & 62 \\
\hline 06 & 43 & 58 & 51 & 65 & 60 & 49 & 58 \\
\hline 07 & 40 & 58 & 52 & 84 & 56 & 41 & 51 \\
\hline 08 & 53 & 68 & 53 & 58 & 81 & 70 & 75 \\
\hline 09 & 47 & 55 & 44 & 43 & 70 & 74 & 79 \\
\hline DS & 47 & 55 & 48 & 53 & 72 & 80 & - \\
\hline
\end{tabular}

Table B-97. Nevada Site 4 Right, Short Wavelengths.

\begin{tabular}{|c|c|c|c|c|c|c|c|}
\hline $\begin{array}{c}\text { Correlation } \\
\text { Reference }\end{array}$ & 03 & 04 & 06 & 07 & 08 & 09 & DS \\
\hline 03 & 22 & 20 & 13 & 11 & 25 & 11 & 12 \\
\hline 04 & 15 & 38 & 23 & 22 & 28 & 10 & 12 \\
\hline 06 & 9 & 16 & 30 & 12 & 18 & 5 & 12 \\
\hline 07 & 10 & 21 & 18 & 48 & 14 & 9 & 7 \\
\hline 08 & 17 & 23 & 24 & 11 & 55 & 15 & 25 \\
\hline 09 & 11 & 12 & 11 & 9 & 18 & 38 & 36 \\
\hline DS & 9 & 11 & 15 & 5 & 20 & 36 & - \\
\hline
\end{tabular}


Table B-98. Nevada Site 5 Left, IRI Filter.

\begin{tabular}{|c|c|c|c|c|c|c|c|}
\hline $\begin{array}{c}\text { Correlation } \\
\text { Reference }\end{array}$ & \multicolumn{6}{|c|}{ Correlated Device } \\
\hline 03 & 45 & 49 & 45 & 41 & 52 & 47 & 47 \\
\hline 04 & 47 & 56 & 53 & 48 & 57 & 51 & 50 \\
\hline 06 & 43 & 51 & 61 & 60 & 71 & 48 & 47 \\
\hline 07 & 38 & 44 & 53 & 81 & 64 & 35 & 36 \\
\hline 08 & 48 & 53 & 63 & 66 & 93 & 64 & 64 \\
\hline 09 & 45 & 48 & 47 & 37 & 65 & 89 & 91 \\
\hline DS & 45 & 48 & 46 & 36 & 65 & 91 & - \\
\hline
\end{tabular}

Table B-99. Nevada Site 5 Left, Long Wavelengths.

\begin{tabular}{|c|c|c|c|c|c|c|c|}
\hline $\begin{array}{c}\text { Correlation } \\
\text { Reference }\end{array}$ & \multicolumn{6}{|c|}{ Correlated Device } \\
\hline 03 & 86 & 74 & 72 & 72 & 79 & 77 & 76 \\
\hline 04 & 75 & 80 & 61 & 58 & 64 & 65 & 64 \\
\hline 06 & 80 & 69 & 80 & 69 & 88 & 88 & 87 \\
\hline 07 & 72 & 58 & 60 & 95 & 79 & 72 & 74 \\
\hline 08 & 78 & 64 & 77 & 79 & 98 & 94 & 95 \\
\hline 09 & 76 & 64 & 77 & 72 & 94 & 97 & 97 \\
\hline DS & 75 & 63 & 76 & 74 & 95 & 97 & - \\
\hline
\end{tabular}

Table B-100. Nevada Site 5 Left, Medium Wavelengths.

\begin{tabular}{|c|c|c|c|c|c|c|c|}
\hline $\begin{array}{c}\text { Correlation } \\
\text { Reference }\end{array}$ & \multicolumn{7}{|c|}{ Correlated Device } \\
\hline 03 & 30 & 37 & 32 & 31 & 42 & 37 & 36 \\
\hline 04 & 33 & 43 & 41 & 42 & 49 & 43 & 42 \\
\hline 06 & 28 & 38 & 50 & 58 & 65 & 37 & 37 \\
\hline 07 & 25 & 35 & 48 & 75 & 59 & 29 & 30 \\
\hline 08 & 35 & 42 & 56 & 61 & 92 & 57 & 58 \\
\hline 09 & 32 & 38 & 36 & 30 & 59 & 85 & 88 \\
\hline DS & 32 & 37 & 35 & 31 & 59 & 88 & - \\
\hline
\end{tabular}

Table B-101. Nevada Site 5 Left, Short Wavelengths.

\begin{tabular}{|c|c|c|c|c|c|c|c|}
\hline $\begin{array}{c}\text { Correlation } \\
\text { Reference }\end{array}$ & \multicolumn{7}{|c|}{ Correlated Device } \\
\hline 03 & 12 & 10 & 6 & 6 & 11 & 8 & 8 \\
\hline 04 & 7 & 13 & 10 & 11 & 8 & 7 & 7 \\
\hline 06 & 4 & 6 & 22 & 13 & 16 & 4 & 7 \\
\hline 07 & 4 & 8 & 20 & 34 & 12 & 5 & 8 \\
\hline 08 & 8 & 7 & 21 & 9 & 63 & 11 & 22 \\
\hline 09 & 5 & 5 & 10 & 8 & 16 & 27 & 30 \\
\hline DS & 6 & 4 & 11 & 8 & 27 & 30 & - \\
\hline
\end{tabular}


Table B-102. Nevada Site 5 Right, IRI Filter.

\begin{tabular}{|c|c|c|c|c|c|c|c|}
\hline $\begin{array}{c}\text { Correlation } \\
\text { Reference }\end{array}$ & \multicolumn{6}{|c|}{ Correlated Device } \\
\hline 03 & 53 & 56 & 51 & 48 & 44 & 50 & 51 \\
\hline 04 & 53 & 64 & 59 & 56 & 47 & 52 & 54 \\
\hline 06 & 49 & 58 & 69 & 68 & 59 & 57 & 57 \\
\hline 07 & 44 & 52 & 60 & 83 & 63 & 46 & 45 \\
\hline 08 & 41 & 44 & 52 & 62 & 93 & 61 & 59 \\
\hline 09 & 46 & 49 & 55 & 48 & 63 & 91 & 93 \\
\hline DS & 48 & 50 & 54 & 47 & 60 & 93 & - \\
\hline
\end{tabular}

Table B-103. Nevada Site 5 Right, Long Wavelengths.

\begin{tabular}{|c|c|c|c|c|c|c|c|}
\hline $\begin{array}{c}\text { Correlation } \\
\text { Reference }\end{array}$ & \multicolumn{6}{|c|}{ Correlated Device } \\
\hline 03 & 90 & 77 & 74 & 75 & 63 & 72 & 75 \\
\hline 04 & 77 & 82 & 62 & 60 & 53 & 61 & 64 \\
\hline 06 & 84 & 72 & 82 & 71 & 69 & 81 & 84 \\
\hline 07 & 75 & 60 & 62 & 93 & 71 & 78 & 80 \\
\hline 08 & 63 & 52 & 60 & 72 & 97 & 84 & 81 \\
\hline 09 & 72 & 61 & 71 & 78 & 85 & 94 & 96 \\
\hline DS & 74 & 63 & 73 & 80 & 82 & 96 & - \\
\hline
\end{tabular}

Table B-104. Nevada Site 5 Right, Medium Wavelengths.

\begin{tabular}{|c|c|c|c|c|c|c|c|}
\hline $\begin{array}{c}\text { Correlation } \\
\text { Reference }\end{array}$ & 03 & 04 & 06 & 07 & 08 & 09 & DS \\
\hline 03 & 36 & 40 & 35 & 36 & 36 & 40 & 40 \\
\hline 04 & 36 & 50 & 46 & 49 & 41 & 43 & 44 \\
\hline 06 & 32 & 43 & 57 & 67 & 56 & 42 & 42 \\
\hline 07 & 29 & 41 & 55 & 77 & 56 & 35 & 34 \\
\hline 08 & 30 & 34 & 45 & 55 & 90 & 53 & 51 \\
\hline 09 & 33 & 38 & 39 & 36 & 55 & 89 & 91 \\
\hline DS & 34 & 39 & 38 & 35 & 53 & 91 & - \\
\hline
\end{tabular}

Table B-105. Nevada Site 5 Right, Short Wavelengths.

\begin{tabular}{|c|c|c|c|c|c|c|c|}
\hline $\begin{array}{c}\text { Correlation } \\
\text { Reference }\end{array}$ & \multicolumn{7}{|c|}{ Correlated Device } \\
\hline 03 & 16 & 13 & 10 & 11 & 12 & 12 & 12 \\
\hline 04 & 10 & 21 & 14 & 19 & 11 & 10 & 11 \\
\hline 06 & 6 & 10 & 33 & 15 & 16 & 5 & 9 \\
\hline 07 & 8 & 13 & 24 & 39 & 13 & 11 & 17 \\
\hline 08 & 9 & 8 & 21 & 10 & 61 & 11 & 15 \\
\hline 09 & 7 & 8 & 12 & 13 & 16 & 39 & 40 \\
\hline DS & 6 & 9 & 13 & 12 & 19 & 41 & - \\
\hline
\end{tabular}


Table B-106. Nevada Site 6 Left, IRI Filter.

\begin{tabular}{|c|c|c|c|c|c|c|c|}
\hline $\begin{array}{c}\text { Correlation } \\
\text { Reference }\end{array}$ & \multicolumn{6}{|c|}{ Correlated Device } \\
\hline 03 & 28 & 33 & 31 & 32 & 39 & 33 & 37 \\
\hline 04 & 31 & 48 & 40 & 37 & 45 & 43 & 48 \\
\hline 06 & 27 & 35 & 41 & 47 & 55 & 47 & 51 \\
\hline 07 & 28 & 33 & 43 & 82 & 73 & 49 & 52 \\
\hline 08 & 34 & 41 & 51 & 75 & 87 & 66 & 70 \\
\hline 09 & 29 & 38 & 43 & 51 & 68 & 80 & 79 \\
\hline DS & 34 & 44 & 48 & 54 & 72 & 79 & - \\
\hline
\end{tabular}

Table B-107. Nevada Site 6 Left, Long Wavelengths.

\begin{tabular}{|c|c|c|c|c|c|c|c|}
\hline $\begin{array}{c}\text { Correlation } \\
\text { Reference }\end{array}$ & 03 & 04 & 06 & 07 & 08 & 09 & DS \\
\hline 03 & 57 & 13 & 55 & 54 & 58 & 56 & 57 \\
\hline 04 & 13 & 41 & 15 & 9 & 13 & 13 & 15 \\
\hline 06 & 54 & 15 & 62 & 52 & 70 & 67 & 70 \\
\hline 07 & 53 & 9 & 51 & 91 & 76 & 67 & 62 \\
\hline 08 & 58 & 13 & 69 & 76 & 94 & 83 & 86 \\
\hline 09 & 55 & 13 & 66 & 68 & 84 & 84 & 86 \\
\hline DS & 57 & 15 & 70 & 63 & 86 & 86 & - \\
\hline
\end{tabular}

Table B-108. Nevada Site 6 Left, Medium Wavelengths.

\begin{tabular}{|c|c|c|c|c|c|c|c|}
\hline $\begin{array}{c}\text { Correlation } \\
\text { Reference }\end{array}$ & 03 & 04 & 06 & 07 & 08 & 09 & DS \\
\hline 03 & 24 & 31 & 28 & 29 & 37 & 31 & 34 \\
\hline 04 & 27 & 42 & 41 & 40 & 50 & 46 & 50 \\
\hline 06 & 23 & 33 & 40 & 49 & 55 & 44 & 48 \\
\hline 07 & 24 & 33 & 44 & 80 & 68 & 42 & 48 \\
\hline 08 & 31 & 41 & 51 & 71 & 83 & 60 & 67 \\
\hline 09 & 26 & 37 & 42 & 44 & 62 & 76 & 78 \\
\hline DS & 30 & 42 & 47 & 50 & 70 & 78 & - \\
\hline
\end{tabular}

Table B-109. Nevada Site 6 Left, Short Wavelengths.

\begin{tabular}{|c|c|c|c|c|c|c|c|}
\hline $\begin{array}{c}\text { Correlation } \\
\text { Reference }\end{array}$ & \multicolumn{7}{|c|}{ Correlated Device } \\
\hline 03 & 15 & 12 & 12 & 12 & 19 & 10 & 14 \\
\hline 04 & 10 & 14 & 16 & 16 & 21 & 8 & 14 \\
\hline 06 & 6 & 9 & 31 & 20 & 21 & 5 & 13 \\
\hline 07 & 8 & 11 & 29 & 52 & 31 & 4 & 10 \\
\hline 08 & 13 & 15 & 30 & 38 & 44 & 10 & 26 \\
\hline 09 & 9 & 10 & 16 & 7 & 15 & 22 & 22 \\
\hline DS & 10 & 11 & 22 & 12 & 24 & 22 & - \\
\hline
\end{tabular}


Table B-110. Nevada Site 6 Right, IRI Filter.

\begin{tabular}{|c|c|c|c|c|c|c|c|}
\hline $\begin{array}{c}\text { Correlation } \\
\text { Reference }\end{array}$ & \multicolumn{6}{|c|}{ Correlated Device } \\
\hline 03 & 43 & 48 & 45 & 48 & 42 & 46 & 48 \\
\hline 04 & 44 & 63 & 56 & 61 & 53 & 56 & 60 \\
\hline 06 & 39 & 50 & 56 & 65 & 56 & 55 & 59 \\
\hline 07 & 42 & 54 & 60 & 86 & 72 & 59 & 62 \\
\hline 08 & 37 & 47 & 51 & 70 & 87 & 70 & 68 \\
\hline 09 & 40 & 49 & 51 & 61 & 73 & 89 & 90 \\
\hline DS & 42 & 54 & 55 & 64 & 70 & 90 & - \\
\hline
\end{tabular}

Table B-111. Nevada Site 6 Right, Long Wavelengths.

\begin{tabular}{|c|c|c|c|c|c|c|c|}
\hline $\begin{array}{c}\text { Correlation } \\
\text { Reference }\end{array}$ & \multicolumn{6}{|c|}{ Correlated Device } \\
\hline 03 & 61 & 21 & 53 & 58 & 49 & 52 & 52 \\
\hline 04 & 22 & 44 & 24 & 18 & 22 & 23 & 23 \\
\hline 06 & 52 & 24 & 65 & 53 & 62 & 68 & 67 \\
\hline 07 & 57 & 18 & 53 & 90 & 68 & 66 & 72 \\
\hline 08 & 48 & 21 & 62 & 68 & 95 & 86 & 87 \\
\hline 09 & 51 & 23 & 67 & 66 & 86 & 91 & 90 \\
\hline DS & 52 & 23 & 67 & 72 & 87 & 90 & - \\
\hline
\end{tabular}

Table B-112. Nevada Site 6 Right, Medium Wavelengths.

\begin{tabular}{|c|c|c|c|c|c|c|c|}
\hline $\begin{array}{c}\text { Correlation } \\
\text { Reference }\end{array}$ & 03 & 04 & 06 & 07 & 08 & 09 & DS \\
\hline 03 & 39 & 45 & 42 & 46 & 41 & 44 & 46 \\
\hline 04 & 39 & 58 & 56 & 63 & 55 & 57 & 60 \\
\hline 06 & 34 & 46 & 55 & 65 & 56 & 52 & 56 \\
\hline 07 & 37 & 52 & 60 & 85 & 69 & 55 & 58 \\
\hline 08 & 34 & 45 & 52 & 69 & 84 & 67 & 66 \\
\hline 09 & 35 & 47 & 50 & 57 & 71 & 86 & 88 \\
\hline DS & 37 & 51 & 53 & 60 & 69 & 88 & - \\
\hline
\end{tabular}

Table B-113. Nevada Site 6 Right, Short Wavelengths.

\begin{tabular}{|c|c|c|c|c|c|c|c|}
\hline $\begin{array}{c}\text { Correlation } \\
\text { Reference }\end{array}$ & \multicolumn{7}{|c|}{ Correlated Device } \\
\hline 03 & 24 & 22 & 20 & 25 & 24 & 16 & 24 \\
\hline 04 & 18 & 27 & 27 & 32 & 27 & 14 & 25 \\
\hline 06 & 12 & 16 & 46 & 26 & 21 & 8 & 19 \\
\hline 07 & 17 & 23 & 38 & 64 & 31 & 10 & 21 \\
\hline 08 & 17 & 21 & 31 & 33 & 47 & 17 & 30 \\
\hline 09 & 15 & 17 & 24 & 14 & 24 & 29 & 34 \\
\hline DS & 16 & 19 & 29 & 18 & 26 & 34 & - \\
\hline
\end{tabular}


Table B-114. Pennsylvania Site 1 Left, IRI Filter.

\begin{tabular}{|c|c|c|c|c|c|c|c|c|c|c|c|c|c|}
\hline $\begin{array}{c}\text { Correlation } \\
\text { Reference }\end{array}$ & 01 & 02 & 03 & 04 & 05 & 06 & 07 & 08 & 73 & 74 & 75 & 76 & DS \\
\hline 01 & $\mathbf{9 6}$ & 80 & 80 & 90 & 80 & 85 & 91 & 89 & 88 & 90 & 88 & 88 & 86 \\
\hline 02 & 82 & 90 & 77 & 76 & 86 & 79 & 86 & 79 & 84 & 82 & 86 & 80 & 77 \\
\hline 03 & 85 & 82 & 89 & 85 & 78 & 82 & 85 & 80 & 85 & 84 & 82 & 86 & 76 \\
\hline 04 & 91 & 80 & 86 & $\mathbf{9 6}$ & 77 & 87 & 86 & 86 & 85 & 90 & 82 & 89 & 80 \\
\hline 05 & 80 & 82 & 72 & 75 & 92 & 76 & 84 & 77 & 81 & 76 & 81 & 77 & 73 \\
\hline 06 & 91 & 86 & 84 & 88 & 82 & 90 & 89 & 87 & 90 & 90 & 86 & 89 & 80 \\
\hline 07 & 91 & 80 & 77 & 84 & 84 & 82 & 93 & 85 & 87 & 84 & 87 & 85 & 85 \\
\hline 08 & 90 & 79 & 76 & 86 & 79 & 84 & 88 & 88 & 87 & 84 & 85 & 84 & 80 \\
\hline 73 & 89 & 83 & 80 & 83 & 83 & 84 & 91 & 85 & 92 & 85 & 90 & 85 & 84 \\
\hline 74 & 92 & 84 & 80 & 86 & 81 & 84 & 91 & 86 & 89 & 92 & 90 & 89 & 87 \\
\hline 75 & 87 & 80 & 76 & 79 & 81 & 80 & 87 & 82 & 86 & 82 & 90 & 81 & 88 \\
\hline 76 & 91 & 82 & 82 & 86 & 81 & 83 & 90 & 84 & 88 & 89 & 88 & 91 & 84 \\
\hline DS & 84 & 70 & 69 & 77 & 72 & 72 & 83 & 77 & 76 & 78 & 80 & 77 & - \\
\hline
\end{tabular}

Table B-115. Pennsylvania Site 1 Left, Long Wavelengths.

\begin{tabular}{|c|c|c|c|c|c|c|c|c|c|c|c|c|c|}
\hline $\begin{array}{c}\text { Correlation } \\
\text { Reference }\end{array}$ & 01 & 02 & 03 & 04 & 05 & 06 & 07 & 08 & 73 & 74 & 75 & 76 & DS \\
\hline 01 & 96 & 67 & 73 & 87 & 78 & 77 & 84 & 81 & 81 & 84 & 83 & 84 & 89 \\
\hline 02 & 68 & 89 & 84 & 63 & 83 & 86 & 77 & 72 & 86 & 83 & 83 & 82 & 63 \\
\hline 03 & 73 & 84 & 89 & 69 & 82 & 82 & 80 & 78 & 83 & 83 & 82 & 83 & 69 \\
\hline 04 & 87 & 62 & 69 & 82 & 70 & 71 & 75 & 76 & 74 & 76 & 76 & 77 & 82 \\
\hline 05 & 78 & 83 & 82 & 70 & 99 & 88 & 92 & 74 & 92 & 94 & 92 & 94 & 72 \\
\hline 06 & 77 & 86 & 82 & 71 & 89 & 94 & 84 & 75 & 95 & 92 & 92 & 91 & 71 \\
\hline 07 & 84 & 77 & 80 & 75 & 92 & 83 & 96 & 78 & 86 & 89 & 88 & 89 & 78 \\
\hline 08 & 82 & 72 & 78 & 76 & 74 & 74 & 78 & 81 & 77 & 79 & 79 & 80 & 73 \\
\hline 73 & 81 & 86 & 83 & 74 & 92 & 94 & 87 & 78 & 97 & 96 & 96 & 95 & 74 \\
\hline 74 & 84 & 82 & 83 & 77 & 94 & 91 & 90 & 80 & 95 & 97 & 95 & 97 & 76 \\
\hline 75 & 83 & 83 & 82 & 76 & 92 & 92 & 88 & 79 & 95 & 96 & 95 & 95 & 75 \\
\hline 76 & 85 & 81 & 83 & 78 & 93 & 90 & 90 & 80 & 94 & 97 & 95 & 97 & 77 \\
\hline DS & 89 & 63 & 68 & 82 & 72 & 70 & 78 & 73 & 74 & 76 & 75 & 77 & - \\
\hline
\end{tabular}


Table B-116. Pennsylvania Site 1 Left, Medium Wavelengths.

\begin{tabular}{|c|c|c|c|c|c|c|c|c|c|c|c|c|c|}
\hline $\begin{array}{c}\text { Correlation } \\
\text { Reference }\end{array}$ & 01 & 02 & 03 & 04 & 05 & 06 & 07 & 08 & 73 & 74 & 75 & 76 & DS \\
\hline 01 & 95 & 75 & 77 & 88 & 75 & 85 & 90 & 86 & 86 & 88 & 83 & 86 & 84 \\
\hline 02 & 73 & 85 & 67 & 69 & 81 & 70 & 77 & 70 & 76 & 74 & 77 & 71 & 75 \\
\hline 03 & 80 & 74 & 84 & 80 & 72 & 75 & 81 & 71 & 79 & 78 & 77 & 79 & 76 \\
\hline 04 & 89 & 74 & 84 & 96 & 72 & 86 & 85 & 83 & 80 & 86 & 77 & 85 & 77 \\
\hline 05 & 74 & 76 & 64 & 69 & 89 & 69 & 77 & 70 & 73 & 67 & 74 & 68 & 68 \\
\hline 06 & 89 & 79 & 77 & 82 & 77 & 86 & 88 & 81 & 87 & 85 & 83 & 83 & 81 \\
\hline 07 & 89 & 76 & 73 & 82 & 77 & 80 & 88 & 81 & 85 & 80 & 82 & 81 & 82 \\
\hline 08 & 87 & 73 & 72 & 83 & 72 & 82 & 83 & 85 & 82 & 80 & 79 & 79 & 75 \\
\hline 73 & 82 & 77 & 73 & 77 & 77 & 79 & 84 & 76 & 88 & 79 & 84 & 78 & 82 \\
\hline 74 & 85 & 78 & 74 & 80 & 76 & 78 & 86 & 77 & 83 & 89 & 84 & 83 & 88 \\
\hline 75 & 80 & 74 & 69 & 73 & 76 & 74 & 81 & 73 & 80 & 75 & 84 & 73 & 82 \\
\hline 76 & 85 & 75 & 76 & 81 & 75 & 77 & 85 & 75 & 82 & 83 & 81 & 86 & 83 \\
\hline DS & 80 & 68 & 65 & 73 & 69 & 69 & 80 & 71 & 72 & 75 & 78 & 73 & - \\
\hline
\end{tabular}

Table B-117. Pennsylvania Site 1 Left, Short Wavelengths.

\begin{tabular}{|c|c|c|c|c|c|c|c|c|c|c|c|c|c|}
\hline $\begin{array}{c}\text { Correlation } \\
\text { Reference }\end{array}$ & 01 & 02 & 03 & 04 & 05 & 06 & 07 & 08 & 73 & 74 & 75 & 76 & DS \\
\hline 01 & 88 & 52 & 44 & 61 & 55 & 60 & 64 & 68 & 70 & 70 & 60 & 66 & 68 \\
\hline 02 & 39 & 66 & 42 & 38 & 52 & 41 & 41 & 37 & 51 & 51 & 52 & 51 & 38 \\
\hline 03 & 34 & 43 & 65 & 39 & 40 & 41 & 35 & 29 & 52 & 50 & 52 & 51 & 31 \\
\hline 04 & 72 & 61 & 60 & 79 & 61 & 63 & 65 & 57 & 70 & 75 & 65 & 73 & 58 \\
\hline 05 & 50 & 49 & 43 & 42 & 81 & 47 & 54 & 47 & 52 & 50 & 53 & 46 & 37 \\
\hline 06 & 51 & 44 & 45 & 43 & 48 & 64 & 45 & 46 & 65 & 61 & 61 & 58 & 40 \\
\hline 07 & 64 & 48 & 43 & 51 & 54 & 56 & 60 & 56 & 62 & 58 & 58 & 60 & 47 \\
\hline 08 & 73 & 49 & 40 & 50 & 55 & 61 & 57 & 74 & 65 & 59 & 63 & 58 & 53 \\
\hline 73 & 45 & 45 & 45 & 39 & 44 & 52 & 42 & 42 & 75 & 55 & 58 & 54 & 37 \\
\hline 74 & 48 & 45 & 43 & 40 & 43 & 49 & 43 & 43 & 55 & 75 & 55 & 58 & 44 \\
\hline 75 & 49 & 45 & 44 & 37 & 47 & 49 & 42 & 43 & 57 & 54 & 69 & 50 & 48 \\
\hline 76 & 45 & 47 & 45 & 42 & 44 & 48 & 42 & 38 & 56 & 60 & 52 & 68 & 42 \\
\hline DS & 50 & 39 & 34 & 34 & 40 & 38 & 41 & 41 & 45 & 54 & 56 & 49 & - \\
\hline
\end{tabular}


Table B-118. Pennsylvania Site 1 Right, IRI Filter

\begin{tabular}{|c|c|c|c|c|c|c|c|c|c|c|c|c|c|}
\hline $\begin{array}{c}\text { Correlation } \\
\text { Reference }\end{array}$ & 01 & 02 & 03 & 04 & 05 & 06 & 07 & 08 & 73 & 74 & 75 & 76 & DS \\
\hline 01 & $\mathbf{9 4}$ & 81 & 77 & 86 & 86 & 83 & 87 & 92 & 90 & 89 & 89 & 86 & 89 \\
\hline 02 & 86 & 88 & 76 & 76 & 80 & 78 & 80 & 85 & 85 & 83 & 85 & 81 & 78 \\
\hline 03 & 84 & 81 & 89 & 87 & 88 & 83 & 88 & 80 & 86 & 86 & 83 & 87 & 78 \\
\hline 04 & 87 & 78 & 85 & $\mathbf{9 5}$ & 90 & 84 & 92 & 83 & 86 & 85 & 81 & 89 & 80 \\
\hline 05 & 87 & 82 & 86 & 91 & $\mathbf{9 5}$ & 87 & $\mathbf{9 4}$ & 82 & 90 & 88 & 85 & 92 & 81 \\
\hline 06 & 89 & 84 & 85 & 87 & 89 & 89 & 90 & 85 & 90 & 89 & 86 & 90 & 80 \\
\hline 07 & 89 & 81 & 84 & 92 & $\mathbf{9 4}$ & 86 & $\mathbf{9 6}$ & 84 & 90 & 89 & 86 & 91 & 83 \\
\hline 08 & 92 & 80 & 74 & 81 & 81 & 79 & 83 & 92 & 86 & 83 & 86 & 82 & 86 \\
\hline 73 & 92 & 84 & 81 & 83 & 85 & 84 & 86 & 89 & 92 & 88 & 90 & 86 & 85 \\
\hline 74 & 91 & 82 & 79 & 83 & 85 & 82 & 85 & 88 & 87 & 91 & 89 & 88 & 88 \\
\hline 75 & 89 & 80 & 76 & 78 & 80 & 78 & 81 & 86 & 85 & 84 & 90 & 80 & 89 \\
\hline 76 & 90 & 81 & 82 & 87 & 87 & 83 & 88 & 87 & 89 & 90 & 88 & 92 & 86 \\
\hline DS & 85 & 71 & 71 & 78 & 78 & 73 & 79 & 83 & 78 & 79 & 83 & 78 & - \\
\hline
\end{tabular}

Table B-119. Pennsylvania Site 1 Right, Long Wavelengths.

\begin{tabular}{|c|c|c|c|c|c|c|c|c|c|c|c|c|c|}
\hline $\begin{array}{c}\text { Correlation } \\
\text { Reference }\end{array}$ & 01 & 02 & 03 & 04 & 05 & 06 & 07 & 08 & 73 & 74 & 75 & 76 & DS \\
\hline 01 & 98 & 84 & 78 & 88 & 87 & 79 & 85 & 95 & 84 & 84 & 87 & 83 & 90 \\
\hline 02 & 84 & 94 & 89 & 77 & 89 & 88 & 89 & 83 & 93 & 92 & 94 & 91 & 77 \\
\hline 03 & 78 & 90 & 92 & 72 & 84 & 89 & 85 & 77 & 91 & 90 & 89 & 91 & 72 \\
\hline 04 & 88 & 77 & 72 & 82 & 80 & 72 & 78 & 85 & 77 & 77 & 79 & 76 & 84 \\
\hline 05 & 87 & 89 & 84 & 80 & 99 & 85 & 98 & 83 & 89 & 89 & 91 & 88 & 80 \\
\hline 06 & 80 & 89 & 90 & 73 & 85 & 96 & 85 & 77 & 94 & 94 & 91 & 95 & 73 \\
\hline 07 & 84 & 89 & 84 & 78 & 98 & 85 & 98 & 81 & 90 & 89 & 91 & 88 & 78 \\
\hline 08 & 95 & 82 & 77 & 85 & 83 & 76 & 81 & 93 & 82 & 81 & 84 & 80 & 87 \\
\hline 73 & 85 & 93 & 91 & 77 & 89 & 94 & 90 & 82 & 98 & 97 & 96 & 97 & 77 \\
\hline 74 & 85 & 92 & 90 & 77 & 89 & 94 & 89 & 82 & 97 & 97 & 96 & 97 & 77 \\
\hline 75 & 87 & 94 & 88 & 79 & 91 & 90 & 91 & 85 & 96 & 95 & 97 & 94 & 79 \\
\hline 76 & 84 & 92 & 91 & 76 & 88 & 94 & 89 & 81 & 97 & 97 & 95 & 97 & 76 \\
\hline DS & 89 & 77 & 72 & 84 & 80 & 72 & 78 & 86 & 77 & 77 & 79 & 76 & - \\
\hline
\end{tabular}


Table B-120. Pennsylvania Site 1 Right, Medium Wavelengths.

\begin{tabular}{|c|c|c|c|c|c|c|c|c|c|c|c|c|c|}
\hline $\begin{array}{c}\text { Correlation } \\
\text { Reference }\end{array}$ & 01 & 02 & 03 & 04 & 05 & 06 & 07 & 08 & 73 & 74 & 75 & 76 & DS \\
\hline 01 & 94 & 76 & 73 & 82 & 83 & 83 & 86 & 90 & 87 & 86 & 83 & 83 & 87 \\
\hline 02 & 79 & 83 & 66 & 67 & 70 & 71 & 72 & 77 & 77 & 76 & 79 & 72 & 76 \\
\hline 03 & 79 & 73 & 85 & 81 & 81 & 78 & 82 & 76 & 81 & 81 & 78 & 81 & 78 \\
\hline 04 & 83 & 71 & 84 & 95 & 90 & 83 & 92 & 80 & 81 & 78 & 74 & 84 & 77 \\
\hline 05 & 85 & 75 & 83 & 89 & 92 & 86 & 91 & 80 & 86 & 83 & 79 & 88 & 80 \\
\hline 06 & 89 & 78 & 77 & 81 & 82 & 84 & 85 & 85 & 86 & 85 & 83 & 84 & 83 \\
\hline 07 & 89 & 76 & 80 & 89 & 91 & 85 & 94 & 84 & 88 & 85 & 81 & 90 & 83 \\
\hline 08 & 91 & 76 & 70 & 77 & 78 & 79 & 82 & 90 & 85 & 81 & 82 & 81 & 82 \\
\hline 73 & 86 & 76 & 73 & 75 & 77 & 79 & 80 & 82 & 88 & 81 & 84 & 79 & 85 \\
\hline 74 & 85 & 75 & 72 & 76 & 77 & 77 & 80 & 81 & 80 & 87 & 83 & 81 & 87 \\
\hline 75 & 81 & 72 & 68 & 70 & 71 & 72 & 74 & 78 & 78 & 76 & 85 & 72 & 85 \\
\hline 76 & 84 & 74 & 74 & 80 & 80 & 78 & 82 & 80 & 82 & 84 & 82 & 88 & 88 \\
\hline DS & 82 & 67 & 67 & 73 & 75 & 71 & 78 & 78 & 75 & 77 & 83 & 76 & - \\
\hline
\end{tabular}

Table B-121. Pennsylvania Site 1 Right, Short Wavelengths.

\begin{tabular}{|c|c|c|c|c|c|c|c|c|c|c|c|c|c|}
\hline $\begin{array}{c}\text { Correlation } \\
\text { Reference }\end{array}$ & 01 & 02 & 03 & 04 & 05 & 06 & 07 & 08 & 73 & 74 & 75 & 76 & DS \\
\hline 01 & 85 & 52 & 38 & 51 & 58 & 60 & 70 & 76 & 74 & 67 & 62 & 62 & 74 \\
\hline 02 & 38 & 64 & 32 & 30 & 37 & 39 & 42 & 41 & 45 & 49 & 52 & 45 & 41 \\
\hline 03 & 26 & 35 & 66 & 35 & 41 & 42 & 41 & 28 & 50 & 49 & 52 & 46 & 38 \\
\hline 04 & 60 & 43 & 61 & 80 & 69 & 64 & 77 & 55 & 69 & 60 & 60 & 70 & 51 \\
\hline 05 & 60 & 46 & 56 & 56 & 79 & 61 & 73 & 54 & 69 & 62 & 65 & 68 & 61 \\
\hline 06 & 48 & 43 & 45 & 40 & 46 & 62 & 52 & 51 & 63 & 60 & 64 & 57 & 50 \\
\hline 07 & 66 & 47 & 48 & 55 & 73 & 59 & 81 & 67 & 68 & 62 & 64 & 67 & 65 \\
\hline 08 & 69 & 46 & 33 & 38 & 45 & 52 & 58 & 81 & 63 & 56 & 63 & 55 & 59 \\
\hline 73 & 47 & 43 & 44 & 37 & 45 & 54 & 50 & 49 & 74 & 54 & 62 & 55 & 48 \\
\hline 74 & 46 & 44 & 39 & 34 & 43 & 47 & 48 & 51 & 50 & 70 & 53 & 53 & 45 \\
\hline 75 & 43 & 42 & 38 & 30 & 37 & 45 & 42 & 46 & 52 & 48 & 67 & 45 & 54 \\
\hline 76 & 40 & 43 & 40 & 39 & 46 & 47 & 50 & 42 & 54 & 56 & 53 & 68 & 51 \\
\hline DS & 47 & 39 & 35 & 31 & 43 & 41 & 48 & 49 & 47 & 51 & 62 & 50 & - \\
\hline
\end{tabular}


Table B-122. Pennsylvania Site 2 Left, IRI Filter.

\begin{tabular}{|c|c|c|c|c|c|c|c|c|c|c|c|c|c|}
\hline $\begin{array}{c}\text { Correlation } \\
\text { Reference }\end{array}$ & 01 & 02 & 03 & 04 & 05 & 06 & 07 & 08 & 73 & 74 & 75 & 76 & DS \\
\hline 01 & 92 & 52 & 44 & 71 & 55 & 50 & 78 & 70 & 62 & 78 & 70 & 79 & 60 \\
\hline 02 & 57 & 59 & 45 & 56 & 50 & 47 & 59 & 59 & 58 & 62 & 58 & 55 & 49 \\
\hline 03 & 47 & 48 & 49 & 50 & 34 & 47 & 49 & 46 & 52 & 51 & 50 & 49 & 47 \\
\hline 04 & 72 & 52 & 46 & 71 & 43 & 48 & 68 & 65 & 61 & 69 & 65 & 65 & 59 \\
\hline 05 & 56 & 45 & 31 & 42 & 87 & 31 & 59 & 43 & 43 & 55 & 47 & 51 & 34 \\
\hline 06 & 53 & 49 & 45 & 51 & 34 & 49 & 52 & 53 & 55 & 54 & 55 & 53 & 50 \\
\hline 07 & 79 & 54 & 45 & 67 & 59 & 48 & 82 & 64 & 60 & 76 & 68 & 76 & 56 \\
\hline 08 & 71 & 55 & 43 & 66 & 44 & 49 & 65 & 73 & 61 & 69 & 67 & 65 & 59 \\
\hline 73 & 66 & 54 & 46 & 64 & 46 & 49 & 66 & 64 & 61 & 68 & 66 & 65 & 58 \\
\hline 74 & 82 & 55 & 46 & 68 & 60 & 48 & 78 & 66 & 61 & 79 & 69 & 77 & 56 \\
\hline 75 & 76 & 52 & 44 & 66 & 50 & 49 & 72 & 65 & 61 & 74 & 69 & 74 & 57 \\
\hline 76 & 83 & 48 & 44 & 65 & 54 & 47 & 73 & 62 & 59 & 72 & 67 & 81 & 56 \\
\hline DS & 63 & 45 & 42 & 61 & 35 & 46 & 60 & 62 & 58 & 62 & 62 & 62 & - \\
\hline
\end{tabular}

Table B-123. Pennsylvania Site 2 Left, Long Wavelengths.

\begin{tabular}{|c|c|c|c|c|c|c|c|c|c|c|c|c|c|}
\hline $\begin{array}{c}\text { Correlation } \\
\text { Reference }\end{array}$ & 01 & 02 & 03 & 04 & 05 & 06 & 07 & 08 & 73 & 74 & 75 & 76 & DS \\
\hline 01 & 98 & 85 & 84 & 60 & 77 & 84 & 81 & 92 & 87 & 90 & 90 & 91 & 89 \\
\hline 02 & 85 & 92 & 90 & 59 & 84 & 89 & 82 & 91 & 92 & 93 & 92 & 91 & 77 \\
\hline 03 & 84 & 90 & 89 & 57 & 82 & 88 & 82 & 89 & 90 & 92 & 91 & 91 & 77 \\
\hline 04 & 60 & 59 & 57 & 50 & 46 & 57 & 46 & 63 & 59 & 59 & 58 & 58 & 53 \\
\hline 05 & 76 & 84 & 82 & 46 & 99 & 81 & 93 & 78 & 83 & 86 & 85 & 86 & 71 \\
\hline 06 & 84 & 89 & 88 & 57 & 82 & 87 & 82 & 88 & 90 & 92 & 91 & 91 & 77 \\
\hline 07 & 81 & 81 & 81 & 46 & 93 & 81 & 98 & 76 & 82 & 86 & 85 & 86 & 76 \\
\hline 08 & 92 & 90 & 89 & 63 & 78 & 88 & 77 & 97 & 91 & 94 & 93 & 94 & 81 \\
\hline 73 & 87 & 91 & 90 & 59 & 83 & 90 & 83 & 92 & 92 & 94 & 93 & 94 & 78 \\
\hline 74 & 90 & 93 & 92 & 59 & 87 & 91 & 86 & 94 & 94 & 97 & 96 & 97 & 81 \\
\hline 75 & 90 & 91 & 91 & 58 & 85 & 90 & 86 & 93 & 93 & 96 & 94 & 96 & 82 \\
\hline 76 & 91 & 91 & 91 & 58 & 86 & 91 & 86 & 94 & 93 & 97 & 95 & 97 & 82 \\
\hline DS & 89 & 77 & 77 & 53 & 71 & 77 & 76 & 81 & 78 & 81 & 81 & 82 & - \\
\hline
\end{tabular}


Table B-124. Pennsylvania Site 2 Left, Medium Wavelengths.

\begin{tabular}{|c|c|c|c|c|c|c|c|c|c|c|c|c|c|}
\hline $\begin{array}{c}\text { Correlation } \\
\text { Reference }\end{array}$ & 01 & 02 & 03 & 04 & 05 & 06 & 07 & 08 & 73 & 74 & 75 & 76 & DS \\
\hline 01 & 88 & 40 & 30 & 71 & 46 & 39 & 67 & 59 & 51 & 69 & 60 & 70 & 49 \\
\hline 02 & 47 & 48 & 29 & 47 & 41 & 31 & 50 & 48 & 45 & 49 & 45 & 40 & 37 \\
\hline 03 & 34 & 34 & 35 & 39 & 22 & 32 & 39 & 33 & 38 & 37 & 35 & 34 & 35 \\
\hline 04 & 72 & 41 & 34 & 74 & 42 & 37 & 68 & 56 & 52 & 67 & 59 & 62 & 49 \\
\hline 05 & 45 & 34 & 18 & 39 & 81 & 19 & 43 & 32 & 30 & 43 & 32 & 35 & 23 \\
\hline 06 & 42 & 35 & 30 & 42 & 22 & 36 & 43 & 42 & 41 & 41 & 42 & 40 & 40 \\
\hline 07 & 69 & 43 & 33 & 69 & 43 & 37 & 69 & 56 & 50 & 66 & 58 & 66 & 47 \\
\hline 08 & 61 & 43 & 28 & 56 & 34 & 36 & 57 & 62 & 48 & 55 & 54 & 51 & 47 \\
\hline 73 & 57 & 40 & 31 & 58 & 36 & 35 & 57 & 51 & 48 & 57 & 52 & 52 & 45 \\
\hline 74 & 76 & 41 & 30 & 65 & 50 & 34 & 63 & 52 & 47 & 68 & 55 & 64 & 43 \\
\hline 75 & 68 & 37 & 29 & 63 & 38 & 35 & 60 & 52 & 47 & 61 & 56 & 62 & 43 \\
\hline 76 & 76 & 33 & 28 & 62 & 38 & 33 & 58 & 48 & 44 & 58 & 53 & 70 & 43 \\
\hline DS & 53 & 33 & 29 & 54 & 25 & 34 & 52 & 51 & 46 & 50 & 49 & 50 & - \\
\hline
\end{tabular}

Table B-125. Pennsylvania Site 2 Left, Short Wavelengths.

\begin{tabular}{|c|c|c|c|c|c|c|c|c|c|c|c|c|c|}
\hline Correlation & \multicolumn{13}{|c|}{ Correlated Device } \\
\hline Reference & 01 & 02 & 03 & 04 & 05 & 06 & 07 & 08 & 73 & 74 & 75 & 76 & DS \\
\hline 01 & 58 & 19 & 10 & 20 & 20 & 18 & 12 & 25 & 30 & 40 & 34 & 38 & 23 \\
\hline 02 & 23 & 26 & 11 & 12 & 23 & 12 & 10 & 17 & 23 & 31 & 25 & 25 & 14 \\
\hline 03 & 11 & 16 & 15 & 13 & 11 & 11 & 9 & 11 & 19 & 20 & 16 & 17 & 13 \\
\hline 04 & 30 & 18 & 13 & 26 & 22 & 14 & 13 & 17 & 29 & 42 & 32 & 38 & 23 \\
\hline 05 & 25 & 18 & 8 & 12 & 50 & 9 & 10 & 13 & 19 & 29 & 19 & 22 & 10 \\
\hline 06 & 23 & 17 & 10 & 15 & 13 & 17 & 10 & 18 & 23 & 25 & 25 & 25 & 17 \\
\hline 07 & 16 & 21 & 13 & 18 & 10 & 15 & 18 & 15 & 26 & 33 & 29 & 33 & 17 \\
\hline 08 & 29 & 22 & 9 & 14 & 16 & 15 & 10 & 27 & 24 & 29 & 29 & 27 & 18 \\
\hline 73 & 31 & 17 & 11 & 15 & 18 & 13 & 10 & 15 & 26 & 33 & 28 & 32 & 15 \\
\hline 74 & 30 & 18 & 10 & 14 & 19 & 12 & 9 & 14 & 22 & 40 & 25 & 31 & 15 \\
\hline 75 & 34 & 16 & 9 & 13 & 15 & 12 & 9 & 16 & 23 & 32 & 30 & 31 & 16 \\
\hline 76 & 33 & 14 & 9 & 14 & 14 & 12 & 9 & 14 & 21 & 31 & 25 & 43 & 17 \\
\hline DS & 31 & 14 & 10 & 15 & 14 & 13 & 10 & 17 & 22 & 31 & 27 & 34 & - \\
\hline
\end{tabular}


Table B-126. Pennsylvania Site 2 Right, IRI Filter.

\begin{tabular}{|c|c|c|c|c|c|c|c|c|c|c|c|c|c|}
\hline $\begin{array}{c}\text { Correlation } \\
\text { Reference }\end{array}$ & 01 & 02 & 03 & 04 & 05 & 06 & 07 & 08 & 73 & 74 & 75 & 76 & DS \\
\hline 01 & 88 & 59 & 43 & 66 & 75 & 52 & 72 & 85 & 63 & 76 & 73 & 77 & 75 \\
\hline 02 & 63 & 67 & 48 & 46 & 51 & 54 & 49 & 57 & 62 & 65 & 59 & 54 & 48 \\
\hline 03 & 46 & 51 & 45 & 39 & 41 & 46 & 39 & 43 & 50 & 51 & 46 & 45 & 39 \\
\hline 04 & 66 & 43 & 37 & 60 & 61 & 42 & 60 & 66 & 51 & 58 & 58 & 65 & 62 \\
\hline 05 & 75 & 47 & 38 & 59 & 86 & 45 & 81 & 73 & 55 & 65 & 64 & 76 & 74 \\
\hline 06 & 55 & 53 & 42 & 45 & 49 & 49 & 47 & 53 & 57 & 57 & 55 & 53 & 47 \\
\hline 07 & 72 & 45 & 36 & 60 & 81 & 43 & 83 & 71 & 52 & 62 & 61 & 73 & 69 \\
\hline 08 & 86 & 53 & 39 & 67 & 74 & 49 & 71 & 88 & 59 & 70 & 69 & 78 & 76 \\
\hline 73 & 67 & 57 & 45 & 54 & 59 & 53 & 56 & 63 & 62 & 69 & 65 & 63 & 56 \\
\hline 74 & 80 & 59 & 45 & 61 & 71 & 52 & 68 & 75 & 64 & 75 & 71 & 74 & 67 \\
\hline 75 & 76 & 53 & 41 & 61 & 69 & 50 & 66 & 74 & 61 & 71 & 70 & 73 & 66 \\
\hline 76 & 78 & 49 & 40 & 67 & 79 & 48 & 77 & 78 & 57 & 68 & 67 & 81 & 76 \\
\hline DS & 74 & 43 & 36 & 63 & 71 & 43 & 71 & 74 & 51 & 61 & 61 & 71 & - \\
\hline
\end{tabular}

Table B-127. Pennsylvania Site 2 Right, Long Wavelengths.

\begin{tabular}{|c|c|c|c|c|c|c|c|c|c|c|c|c|c|}
\hline $\begin{array}{c}\text { Correlation } \\
\text { Reference }\end{array}$ & 01 & 02 & 03 & 04 & 05 & 06 & 07 & 08 & 73 & 74 & 75 & 76 & DS \\
\hline 01 & 98 & 88 & 89 & 69 & 80 & 89 & 79 & 97 & 90 & 94 & 93 & 96 & 88 \\
\hline 02 & 88 & 96 & 92 & 65 & 79 & 92 & 78 & 90 & 94 & 93 & 92 & 90 & 79 \\
\hline 03 & 89 & 92 & 91 & 64 & 79 & 92 & 79 & 91 & 93 & 94 & 93 & 92 & 80 \\
\hline 04 & 69 & 65 & 64 & 52 & 54 & 64 & 54 & 69 & 65 & 66 & 66 & 66 & 63 \\
\hline 05 & 80 & 79 & 79 & 55 & 98 & 81 & 98 & 80 & 80 & 83 & 84 & 86 & 81 \\
\hline 06 & 90 & 92 & 92 & 64 & 81 & 92 & 81 & 91 & 93 & 94 & 93 & 92 & 80 \\
\hline 07 & 80 & 79 & 79 & 55 & 98 & 81 & 97 & 79 & 80 & 83 & 83 & 86 & 81 \\
\hline 08 & 97 & 90 & 91 & 69 & 79 & 91 & 79 & 99 & 92 & 96 & 95 & 96 & 87 \\
\hline 73 & 90 & 94 & 93 & 65 & 80 & 93 & 80 & 92 & 94 & 95 & 94 & 93 & 81 \\
\hline 74 & 94 & 93 & 93 & 66 & 84 & 94 & 83 & 96 & 95 & 98 & 97 & 97 & 84 \\
\hline 75 & 93 & 92 & 93 & 66 & 84 & 93 & 83 & 95 & 94 & 97 & 95 & 96 & 84 \\
\hline 76 & 96 & 90 & 91 & 66 & 86 & 92 & 86 & 96 & 92 & 96 & 96 & 98 & 86 \\
\hline DS & 88 & 79 & 80 & 63 & 82 & 80 & 81 & 87 & 80 & 84 & 84 & 86 & - \\
\hline
\end{tabular}


Table B-128. Pennsylvania Site 2 Right, Medium Wavelengths.

\begin{tabular}{|c|c|c|c|c|c|c|c|c|c|c|c|c|c|}
\hline $\begin{array}{c}\text { Correlation } \\
\text { Reference }\end{array}$ & 01 & 02 & 03 & 04 & 05 & 06 & 07 & 08 & 73 & 74 & 75 & 76 & DS \\
\hline 01 & 82 & 43 & 24 & 61 & 64 & 32 & 68 & 75 & 45 & 63 & 56 & 59 & 64 \\
\hline 02 & 49 & 52 & 26 & 32 & 39 & 33 & 36 & 43 & 44 & 50 & 41 & 36 & 36 \\
\hline 03 & 27 & 31 & 26 & 24 & 27 & 26 & 24 & 25 & 29 & 30 & 26 & 25 & 24 \\
\hline 04 & 61 & 28 & 20 & 63 & 58 & 25 & 66 & 59 & 35 & 46 & 44 & 60 & 56 \\
\hline 05 & 65 & 33 & 22 & 57 & 71 & 28 & 63 & 64 & 40 & 53 & 50 & 64 & 64 \\
\hline 06 & 36 & 34 & 22 & 29 & 33 & 29 & 31 & 35 & 36 & 36 & 34 & 32 & 33 \\
\hline 07 & 68 & 31 & 20 & 65 & 63 & 26 & 72 & 64 & 36 & 49 & 46 & 60 & 57 \\
\hline 08 & 76 & 37 & 21 & 60 & 61 & 30 & 66 & 79 & 40 & 55 & 51 & 61 & 63 \\
\hline 73 & 52 & 38 & 24 & 40 & 47 & 31 & 43 & 47 & 43 & 51 & 46 & 44 & 43 \\
\hline 74 & 69 & 41 & 25 & 53 & 59 & 30 & 58 & 63 & 43 & 60 & 51 & 56 & 57 \\
\hline 75 & 62 & 34 & 21 & 51 & 57 & 28 & 54 & 59 & 39 & 52 & 50 & 55 & 55 \\
\hline 76 & 65 & 30 & 21 & 64 & 60 & 27 & 68 & 61 & 36 & 49 & 45 & 63 & 58 \\
\hline DS & 66 & 30 & 20 & 62 & 60 & 27 & 63 & 65 & 36 & 50 & 47 & 59 & - \\
\hline
\end{tabular}

Table B-129. Pennsylvania Site 2 Right, Short Wavelengths.

\begin{tabular}{|c|c|c|c|c|c|c|c|c|c|c|c|c|c|}
\hline $\begin{array}{c}\text { Correlation } \\
\text { Reference }\end{array}$ & 01 & 02 & 03 & 04 & 05 & 06 & 07 & 08 & 73 & 74 & 75 & 76 & DS \\
\hline 01 & 54 & 21 & 8 & 20 & 14 & 11 & 23 & 40 & 24 & 37 & 29 & 32 & 33 \\
\hline 02 & 23 & 26 & 9 & 13 & 11 & 10 & 16 & 21 & 22 & 32 & 25 & 23 & 20 \\
\hline 03 & 9 & 14 & 13 & 9 & 11 & 10 & 10 & 9 & 16 & 17 & 14 & 14 & 11 \\
\hline 04 & 29 & 13 & 8 & 28 & 15 & 10 & 25 & 29 & 19 & 27 & 24 & 36 & 28 \\
\hline 05 & 18 & 15 & 9 & 20 & 27 & 11 & 16 & 16 & 22 & 30 & 27 & 35 & 26 \\
\hline 06 & 14 & 15 & 9 & 13 & 12 & 13 & 15 & 16 & 19 & 20 & 20 & 19 & 16 \\
\hline 07 & 29 & 13 & 8 & 22 & 16 & 10 & 24 & 28 & 19 & 27 & 23 & 33 & 28 \\
\hline 08 & 43 & 17 & 7 & 18 & 12 & 11 & 21 & 45 & 21 & 30 & 27 & 32 & 30 \\
\hline 73 & 26 & 17 & 9 & 18 & 13 & 10 & 20 & 24 & 23 & 31 & 27 & 30 & 25 \\
\hline 74 & 36 & 18 & 9 & 16 & 12 & 9 & 19 & 29 & 21 & 37 & 24 & 30 & 27 \\
\hline 75 & 31 & 15 & 7 & 16 & 12 & 10 & 19 & 29 & 20 & 27 & 27 & 28 & 26 \\
\hline 76 & 32 & 13 & 7 & 20 & 13 & 10 & 21 & 28 & 18 & 27 & 22 & 35 & 25 \\
\hline DS & 36 & 14 & 7 & 18 & 13 & 10 & 21 & 32 & 18 & 27 & 23 & 29 & - \\
\hline
\end{tabular}


Table B-130. Pennsylvania Site 3 Left, IRI Filter.

\begin{tabular}{|c|c|c|c|c|c|c|c|c|c|c|c|c|c|}
\hline $\begin{array}{c}\text { Correlation } \\
\text { Reference }\end{array}$ & 01 & 02 & 03 & 04 & 05 & 06 & 07 & 08 & 73 & 74 & 75 & 76 & DS \\
\hline 01 & 91 & 43 & 41 & 85 & 80 & 30 & 84 & 89 & 40 & 48 & 45 & 45 & 82 \\
\hline 02 & 45 & 48 & 45 & 46 & 44 & 33 & 46 & 46 & 44 & 51 & 48 & 49 & 44 \\
\hline 03 & 43 & 44 & 46 & 46 & 40 & 33 & 42 & 42 & 44 & 48 & 48 & 49 & 44 \\
\hline 04 & 86 & 44 & 44 & 87 & 75 & 30 & 80 & 83 & 42 & 49 & 46 & 47 & 78 \\
\hline 05 & 81 & 41 & 37 & 73 & $\mathbf{9 6}$ & 26 & 90 & 81 & 35 & 43 & 39 & 40 & 75 \\
\hline 06 & 31 & 35 & 35 & 32 & 29 & 25 & 31 & 31 & 34 & 37 & 37 & 37 & 32 \\
\hline 07 & 85 & 42 & 39 & 79 & 90 & 28 & 91 & 85 & 38 & 46 & 41 & 43 & 80 \\
\hline 08 & 90 & 42 & 39 & 82 & 81 & 29 & 85 & 92 & 38 & 47 & 43 & 43 & 85 \\
\hline 73 & 42 & 47 & 47 & 43 & 38 & 33 & 41 & 42 & 47 & 51 & 49 & 51 & 41 \\
\hline 74 & 51 & 48 & 46 & 51 & 47 & 34 & 50 & 51 & 45 & 52 & 50 & 51 & 49 \\
\hline 75 & 47 & 46 & 46 & 48 & 42 & 33 & 45 & 47 & 45 & 51 & 49 & 51 & 47 \\
\hline 76 & 48 & 47 & 48 & 49 & 43 & 34 & 46 & 47 & 46 & 52 & 51 & 51 & 48 \\
\hline DS & 86 & 40 & 39 & 79 & 77 & 28 & 80 & 85 & 37 & 44 & 42 & 43 & - \\
\hline
\end{tabular}

Table B-131. Pennsylvania Site 3 Left, Long Wavelengths.

\begin{tabular}{|c|c|c|c|c|c|c|c|c|c|c|c|c|c|}
\hline Correlation & \multicolumn{13}{|c|}{ Correlated Device } \\
\hline Reference & 01 & 02 & 03 & 04 & 05 & 06 & 07 & 08 & 73 & 74 & 75 & 76 & DS \\
\hline 01 & 97 & 89 & 86 & 84 & 77 & 81 & 77 & 97 & 85 & 87 & 86 & 84 & 94 \\
\hline 02 & 89 & 94 & 92 & 85 & 82 & 88 & 83 & 90 & 92 & 93 & 92 & 91 & 86 \\
\hline 03 & 86 & 93 & 93 & 85 & 80 & 90 & 81 & 86 & 93 & 94 & 94 & 93 & 83 \\
\hline 04 & 84 & 85 & 84 & 86 & 64 & 82 & 64 & 84 & 85 & 86 & 86 & 86 & 78 \\
\hline 05 & 77 & 82 & 80 & 64 & 99 & 75 & 98 & 77 & 77 & 80 & 77 & 77 & 78 \\
\hline 06 & 82 & 89 & 90 & 83 & 75 & 88 & 76 & 81 & 92 & 92 & 92 & 92 & 78 \\
\hline 07 & 78 & 82 & 80 & 64 & 98 & 75 & 98 & 77 & 78 & 80 & 78 & 78 & 79 \\
\hline 08 & 97 & 89 & 85 & 84 & 77 & 81 & 77 & 98 & 84 & 86 & 85 & 84 & 93 \\
\hline 73 & 85 & 92 & 93 & 86 & 78 & 91 & 79 & 85 & 95 & 95 & 95 & 96 & 82 \\
\hline 74 & 87 & 94 & 94 & 86 & 80 & 91 & 81 & 87 & 95 & 96 & 95 & 95 & 84 \\
\hline 75 & 86 & 92 & 94 & 86 & 77 & 92 & 79 & 86 & 95 & 95 & 96 & 96 & 83 \\
\hline 76 & 85 & 92 & 94 & 86 & 78 & 92 & 78 & 84 & 96 & 95 & 96 & 96 & 82 \\
\hline DS & 94 & 85 & 82 & 78 & 77 & 78 & 78 & 93 & 81 & 83 & 82 & 81 & - \\
\hline
\end{tabular}


Table B-132. Pennsylvania Site 3 Left, Medium Wavelengths.

\begin{tabular}{|c|c|c|c|c|c|c|c|c|c|c|c|c|c|}
\hline $\begin{array}{c}\text { Correlation } \\
\text { Reference }\end{array}$ & 01 & 02 & 03 & 04 & 05 & 06 & 07 & 08 & 73 & 74 & 75 & 76 & DS \\
\hline 01 & 87 & 30 & 27 & 78 & 79 & 19 & 82 & 84 & 27 & 34 & 31 & 30 & 73 \\
\hline 02 & 34 & 34 & 30 & 35 & 35 & 21 & 36 & 35 & 28 & 35 & 32 & 32 & 33 \\
\hline 03 & 31 & 29 & 31 & 34 & 30 & 20 & 31 & 31 & 27 & 32 & 30 & 31 & 32 \\
\hline 04 & 80 & 31 & 31 & 84 & 72 & 20 & 78 & 78 & 28 & 35 & 32 & 32 & 71 \\
\hline 05 & 81 & 28 & 25 & 69 & 95 & 17 & 86 & 78 & 23 & 30 & 26 & 27 & 68 \\
\hline 06 & 22 & 23 & 23 & 23 & 21 & 15 & 22 & 22 & 22 & 25 & 24 & 24 & 22 \\
\hline 07 & 85 & 29 & 26 & 74 & 86 & 18 & 86 & 83 & 25 & 32 & 28 & 29 & 73 \\
\hline 08 & 86 & 29 & 25 & 75 & 80 & 18 & 83 & 87 & 25 & 33 & 29 & 29 & 74 \\
\hline 73 & 30 & 32 & 32 & 32 & 28 & 22 & 30 & 31 & 31 & 36 & 34 & 35 & 30 \\
\hline 74 & 39 & 32 & 30 & 40 & 36 & 21 & 39 & 40 & 29 & 36 & 33 & 33 & 37 \\
\hline 75 & 35 & 30 & 29 & 36 & 32 & 20 & 34 & 35 & 29 & 33 & 32 & 33 & 35 \\
\hline 76 & 35 & 31 & 31 & 37 & 32 & 21 & 35 & 35 & 29 & 34 & 33 & 34 & 35 \\
\hline DS & 79 & 26 & 25 & 71 & 73 & 18 & 76 & 76 & 23 & 29 & 27 & 28 & - \\
\hline
\end{tabular}

Table B-133. Pennsylvania Site 3 Left, Short Wavelengths.

\begin{tabular}{|c|c|c|c|c|c|c|c|c|c|c|c|c|c|}
\hline Correlation & \multicolumn{13}{|c|}{ Correlated Device } \\
\hline Reference & 01 & 02 & 03 & 04 & 05 & 06 & 07 & 08 & 73 & 74 & 75 & 76 & DS \\
\hline 01 & 28 & 5 & 4 & 12 & 14 & 3 & 19 & 23 & 5 & 7 & 6 & 5 & 24 \\
\hline 02 & 6 & 12 & 7 & 7 & 6 & 5 & 6 & 6 & 6 & 9 & 8 & 8 & 5 \\
\hline 03 & 4 & 8 & 11 & 7 & 4 & 5 & 4 & 5 & 7 & 8 & 7 & 8 & 7 \\
\hline 04 & 19 & 6 & 5 & 20 & 12 & 3 & 18 & 16 & 5 & 7 & 5 & 6 & 22 \\
\hline 05 & 16 & 4 & 3 & 5 & 59 & 2 & 23 & 16 & 3 & 4 & 3 & 3 & 10 \\
\hline 06 & 4 & 7 & 7 & 4 & 3 & 6 & 3 & 4 & 7 & 8 & 7 & 8 & 5 \\
\hline 07 & 21 & 4 & 3 & 8 & 23 & 2 & 26 & 20 & 4 & 5 & 4 & 4 & 14 \\
\hline 08 & 25 & 4 & 3 & 7 & 20 & 2 & 19 & 30 & 4 & 6 & 5 & 5 & 15 \\
\hline 73 & 5 & 7 & 8 & 6 & 4 & 6 & 5 & 5 & 11 & 9 & 10 & 8 & 6 \\
\hline 74 & 7 & 7 & 7 & 8 & 5 & 4 & 6 & 7 & 6 & 11 & 7 & 8 & 8 \\
\hline 75 & 6 & 7 & 6 & 6 & 4 & 4 & 5 & 6 & 7 & 7 & 11 & 7 & 7 \\
\hline 76 & 6 & 7 & 7 & 7 & 4 & 5 & 5 & 6 & 7 & 8 & 8 & 10 & 8 \\
\hline DS & 19 & 4 & 4 & 9 & 12 & 3 & 18 & 18 & 4 & 5 & 5 & 5 & - \\
\hline
\end{tabular}


Table B-134. Pennsylvania Site 3 Right, IRI Filter.

\begin{tabular}{|c|c|c|c|c|c|c|c|c|c|c|c|c|c|}
\hline $\begin{array}{c}\text { Correlation } \\
\text { Reference }\end{array}$ & 01 & 02 & 03 & 04 & 05 & 06 & 07 & 08 & 73 & 74 & 75 & 76 & DS \\
\hline 01 & 88 & 45 & 55 & 71 & 79 & 46 & 84 & 85 & 48 & 54 & 50 & 44 & 67 \\
\hline 02 & 47 & 53 & 46 & 36 & 42 & 49 & 48 & 45 & 53 & 52 & 48 & 47 & 37 \\
\hline 03 & 57 & 41 & 58 & 51 & 59 & 46 & 60 & 55 & 48 & 54 & 51 & 46 & 50 \\
\hline 04 & 71 & 34 & 49 & 84 & 82 & 39 & 72 & 75 & 40 & 45 & 44 & 39 & 77 \\
\hline 05 & 80 & 39 & 55 & 83 & $\mathbf{9 4}$ & 44 & 86 & 83 & 45 & 52 & 48 & 43 & 78 \\
\hline 06 & 47 & 46 & 51 & 41 & 48 & 48 & 50 & 46 & 51 & 55 & 52 & 49 & 42 \\
\hline 07 & 85 & 45 & 57 & 73 & 86 & 47 & 92 & 82 & 49 & 56 & 51 & 45 & 69 \\
\hline 08 & 87 & 42 & 51 & 75 & 83 & 43 & 82 & 91 & 45 & 52 & 47 & 41 & 74 \\
\hline 73 & 50 & 49 & 53 & 41 & 48 & 51 & 53 & 49 & 53 & 58 & 52 & 50 & 41 \\
\hline 74 & 56 & 47 & 57 & 46 & 55 & 50 & 60 & 55 & 53 & 59 & 54 & 49 & 47 \\
\hline 75 & 52 & 43 & 55 & 46 & 52 & 48 & 54 & 50 & 49 & 55 & 52 & 48 & 46 \\
\hline 76 & 46 & 43 & 52 & 40 & 47 & 49 & 49 & 45 & 50 & 54 & 52 & 48 & 41 \\
\hline DS & 69 & 33 & 46 & 78 & 77 & 38 & 68 & 73 & 37 & 43 & 42 & 37 & - \\
\hline
\end{tabular}

Table B-135. Pennsylvania Site 3 Right, Long Wavelengths.

\begin{tabular}{|c|c|c|c|c|c|c|c|c|c|c|c|c|c|}
\hline $\begin{array}{c}\text { Correlation } \\
\text { Reference }\end{array}$ & 01 & 02 & 03 & 04 & 05 & 06 & 07 & 08 & 73 & 74 & 75 & 76 & DS \\
\hline 01 & 96 & 87 & 91 & 80 & 77 & 92 & 79 & 95 & 91 & 92 & 92 & 93 & 82 \\
\hline 02 & 87 & 94 & 85 & 72 & 76 & 90 & 81 & 84 & 92 & 91 & 86 & 88 & 72 \\
\hline 03 & 91 & 85 & 93 & 79 & 85 & 91 & 83 & 92 & 90 & 90 & 94 & 93 & 83 \\
\hline 04 & 81 & 72 & 79 & 85 & 62 & 77 & 60 & 83 & 76 & 77 & 80 & 79 & 79 \\
\hline 05 & 77 & 76 & 85 & 61 & 99 & 83 & 93 & 79 & 80 & 81 & 85 & 84 & 73 \\
\hline 06 & 92 & 90 & 91 & 76 & 83 & 93 & 86 & 90 & 93 & 94 & 93 & 94 & 79 \\
\hline 07 & 80 & 81 & 83 & 59 & 93 & 86 & 97 & 77 & 85 & 86 & 84 & 86 & 68 \\
\hline 08 & 95 & 83 & 92 & 82 & 79 & 89 & 77 & 97 & 88 & 89 & 93 & 92 & 86 \\
\hline 73 & 91 & 91 & 90 & 76 & 81 & 93 & 85 & 89 & 94 & 94 & 91 & 93 & 77 \\
\hline 74 & 92 & 91 & 91 & 77 & 81 & 94 & 86 & 89 & 94 & 95 & 92 & 94 & 78 \\
\hline 75 & 93 & 86 & 94 & 80 & 85 & 93 & 84 & 94 & 91 & 92 & 95 & 95 & 83 \\
\hline 76 & 93 & 87 & 93 & 79 & 84 & 94 & 86 & 92 & 93 & 94 & 95 & 96 & 81 \\
\hline DS & 82 & 72 & 83 & 79 & 72 & 78 & 68 & 86 & 77 & 77 & 83 & 80 & - \\
\hline
\end{tabular}


Table B-136. Pennsylvania Site 3 Right, Medium Wavelengths.

\begin{tabular}{|c|c|c|c|c|c|c|c|c|c|c|c|c|c|}
\hline $\begin{array}{c}\text { Correlation } \\
\text { Reference }\end{array}$ & 01 & 02 & 03 & 04 & 05 & 06 & 07 & 08 & 73 & 74 & 75 & 76 & DS \\
\hline 01 & 79 & 25 & 29 & 61 & 68 & 24 & 78 & 78 & 27 & 31 & 26 & 22 & 54 \\
\hline 02 & 29 & 32 & 27 & 21 & 25 & 27 & 30 & 30 & 31 & 31 & 28 & 24 & 22 \\
\hline 03 & 34 & 21 & 35 & 34 & 40 & 24 & 40 & 35 & 25 & 29 & 26 & 23 & 33 \\
\hline 04 & 62 & 18 & 30 & 78 & 80 & 23 & 70 & 63 & 23 & 26 & 26 & 22 & 61 \\
\hline 05 & 69 & 21 & 33 & 78 & 88 & 25 & 80 & 71 & 26 & 30 & 27 & 24 & 64 \\
\hline 06 & 28 & 24 & 30 & 26 & 31 & 27 & 32 & 29 & 28 & 32 & 29 & 26 & 27 \\
\hline 07 & 79 & 24 & 33 & 72 & 80 & 26 & 86 & 81 & 28 & 32 & 28 & 24 & 64 \\
\hline 08 & 80 & 24 & 28 & 63 & 73 & 24 & 80 & 85 & 26 & 31 & 25 & 22 & 58 \\
\hline 73 & 31 & 27 & 32 & 26 & 31 & 28 & 34 & 32 & 31 & 34 & 30 & 26 & 26 \\
\hline 74 & 35 & 24 & 33 & 30 & 37 & 27 & 40 & 38 & 28 & 35 & 28 & 25 & 30 \\
\hline 75 & 30 & 22 & 32 & 30 & 34 & 26 & 34 & 31 & 27 & 31 & 30 & 26 & 30 \\
\hline 76 & 26 & 22 & 31 & 26 & 29 & 27 & 30 & 27 & 27 & 31 & 30 & 27 & 25 \\
\hline DS & 57 & 17 & 26 & 65 & 69 & 21 & 63 & 59 & 20 & 24 & 23 & 20 & - \\
\hline
\end{tabular}

Table B-137. Pennsylvania Site 3 Right, Short Wavelengths.

\begin{tabular}{|c|c|c|c|c|c|c|c|c|c|c|c|c|c|}
\hline $\begin{array}{c}\text { Correlation } \\
\text { Reference }\end{array}$ & 01 & 02 & 03 & 04 & 05 & 06 & 07 & 08 & 73 & 74 & 75 & 76 & DS \\
\hline 01 & 39 & 6 & 6 & 13 & 20 & 5 & 30 & 35 & 8 & 9 & 7 & 6 & 13 \\
\hline 02 & 9 & 13 & 10 & 5 & 6 & 7 & 9 & 8 & 9 & 9 & 8 & 7 & 6 \\
\hline 03 & 9 & 6 & 14 & 8 & 11 & 5 & 9 & 8 & 7 & 7 & 7 & 6 & 9 \\
\hline 04 & 16 & 4 & 6 & 28 & 34 & 5 & 29 & 17 & 6 & 6 & 6 & 6 & 12 \\
\hline 05 & 20 & 4 & 7 & 17 & 53 & 4 & 43 & 23 & 7 & 5 & 5 & 5 & 15 \\
\hline 06 & 8 & 7 & 9 & 8 & 9 & 10 & 9 & 8 & 8 & 10 & 9 & 8 & 7 \\
\hline 07 & 31 & 5 & 6 & 15 & 43 & 5 & 51 & 38 & 7 & 7 & 6 & 5 & 14 \\
\hline 08 & 36 & 6 & 5 & 11 & 27 & 5 & 42 & 50 & 7 & 9 & 6 & 5 & 9 \\
\hline 73 & 10 & 7 & 10 & 8 & 10 & 7 & 11 & 10 & 13 & 8 & 10 & 8 & 9 \\
\hline 74 & 12 & 6 & 8 & 8 & 10 & 7 & 13 & 12 & 7 & 16 & 8 & 8 & 9 \\
\hline 75 & 9 & 6 & 9 & 8 & 9 & 6 & 10 & 9 & 9 & 9 & 11 & 8 & 8 \\
\hline 76 & 7 & 6 & 9 & 8 & 8 & 7 & 8 & 6 & 9 & 10 & 9 & 11 & 7 \\
\hline DS & 12 & 3 & 4 & 10 & 22 & 3 & 21 & 12 & 5 & 4 & 4 & 4 & - \\
\hline
\end{tabular}


Table B-138. Pennsylvania Site 4 Left, IRI Filter.

\begin{tabular}{|c|c|c|c|c|c|c|c|c|c|c|c|c|c|}
\hline $\begin{array}{c}\text { Correlation } \\
\text { Reference }\end{array}$ & 01 & 02 & 03 & 04 & 05 & 06 & 07 & 08 & 73 & 74 & 75 & 76 & DS \\
\hline 01 & $\mathbf{9 6}$ & 55 & 53 & 88 & 81 & 57 & 81 & 93 & 52 & 58 & 56 & 58 & 86 \\
\hline 02 & 56 & 61 & 60 & 52 & 55 & 57 & 54 & 58 & 58 & 64 & 62 & 63 & 53 \\
\hline 03 & 54 & 61 & 63 & 52 & 52 & 57 & 52 & 55 & 61 & 65 & 64 & 67 & 51 \\
\hline 04 & 88 & 51 & 50 & 86 & 78 & 53 & 78 & 86 & 50 & 54 & 52 & 55 & 85 \\
\hline 05 & 81 & 52 & 50 & 78 & $\mathbf{9 7}$ & 54 & $\mathbf{9 4}$ & 78 & 49 & 54 & 50 & 54 & 83 \\
\hline 06 & 58 & 56 & 56 & 54 & 56 & 52 & 56 & 59 & 53 & 58 & 56 & 58 & 54 \\
\hline 07 & 82 & 52 & 49 & 78 & $\mathbf{9 4}$ & 54 & 93 & 78 & 49 & 54 & 50 & 54 & 84 \\
\hline 08 & 93 & 55 & 52 & 86 & 79 & 57 & 79 & 93 & 52 & 58 & 56 & 58 & 84 \\
\hline 73 & 54 & 58 & 59 & 51 & 51 & 54 & 51 & 55 & 55 & 62 & 60 & 63 & 51 \\
\hline 74 & 59 & 64 & 63 & 56 & 56 & 58 & 56 & 61 & 61 & 67 & 64 & 67 & 56 \\
\hline 75 & 57 & 62 & 63 & 53 & 52 & 57 & 52 & 59 & 60 & 66 & 63 & 67 & 53 \\
\hline 76 & 60 & 62 & 64 & 56 & 56 & 59 & 56 & 61 & 62 & 67 & 65 & 69 & 56 \\
\hline DS & 87 & 50 & 48 & 86 & 82 & 52 & 83 & 84 & 47 & 53 & 50 & 53 & - \\
\hline
\end{tabular}

Table B-139. Pennsylvania Site 4 Left, Long Wavelengths.

\begin{tabular}{|c|c|c|c|c|c|c|c|c|c|c|c|c|c|}
\hline $\begin{array}{c}\text { Correlation } \\
\text { Reference }\end{array}$ & 01 & 02 & 03 & 04 & 05 & 06 & 07 & 08 & 73 & 74 & 75 & 76 & DS \\
\hline 01 & 98 & 81 & 81 & 88 & 87 & 80 & 87 & 96 & 82 & 83 & 83 & 83 & 89 \\
\hline 02 & 81 & 92 & 91 & 75 & 82 & 90 & 81 & 83 & 92 & 92 & 92 & 92 & 73 \\
\hline 03 & 80 & 91 & 91 & 74 & 80 & 90 & 80 & 82 & 92 & 92 & 92 & 92 & 72 \\
\hline 04 & 88 & 75 & 75 & 84 & 79 & 74 & 79 & 87 & 76 & 76 & 76 & 77 & 87 \\
\hline 05 & 87 & 82 & 80 & 79 & 98 & 80 & 97 & 87 & 82 & 83 & 82 & 82 & 79 \\
\hline 06 & 79 & 90 & 90 & 73 & 80 & 89 & 79 & 81 & 91 & 91 & 90 & 91 & 71 \\
\hline 07 & 88 & 81 & 80 & 79 & 97 & 79 & 97 & 87 & 81 & 82 & 81 & 82 & 80 \\
\hline 08 & 96 & 84 & 82 & 87 & 87 & 82 & 86 & 96 & 84 & 85 & 84 & 84 & 86 \\
\hline 73 & 82 & 92 & 92 & 76 & 82 & 90 & 81 & 83 & 93 & 94 & 93 & 94 & 73 \\
\hline 74 & 82 & 92 & 92 & 76 & 83 & 90 & 82 & 84 & 94 & 94 & 94 & 94 & 74 \\
\hline 75 & 82 & 92 & 92 & 76 & 82 & 90 & 81 & 84 & 93 & 94 & 93 & 94 & 74 \\
\hline 76 & 82 & 92 & 92 & 76 & 82 & 91 & 82 & 84 & 94 & 94 & 94 & 95 & 74 \\
\hline DS & 88 & 73 & 72 & 87 & 79 & 71 & 81 & 86 & 73 & 74 & 74 & 74 & - \\
\hline
\end{tabular}


Table B-140. Pennsylvania Site 4 Left, Medium Wavelengths.

\begin{tabular}{|c|c|c|c|c|c|c|c|c|c|c|c|c|c|}
\hline $\begin{array}{c}\text { Correlation } \\
\text { Reference }\end{array}$ & 01 & 02 & 03 & 04 & 05 & 06 & 07 & 08 & 73 & 74 & 75 & 76 & DS \\
\hline 01 & 85 & 17 & 16 & 73 & 57 & 28 & 59 & 78 & 17 & 21 & 18 & 21 & 74 \\
\hline 02 & 20 & 22 & 19 & 20 & 18 & 21 & 18 & 21 & 19 & 23 & 20 & 20 & 20 \\
\hline 03 & 19 & 20 & 25 & 21 & 16 & 21 & 16 & 19 & 22 & 24 & 22 & 25 & 20 \\
\hline 04 & 75 & 17 & 18 & 77 & 54 & 28 & 56 & 73 & 18 & 21 & 18 & 22 & 69 \\
\hline 05 & 59 & 15 & 13 & 51 & 91 & 22 & 73 & 56 & 12 & 15 & 13 & 15 & 55 \\
\hline 06 & 31 & 17 & 17 & 32 & 26 & 23 & 26 & 31 & 17 & 20 & 17 & 20 & 31 \\
\hline 07 & 61 & 15 & 13 & 53 & 73 & 22 & 69 & 59 & 13 & 16 & 13 & 15 & 58 \\
\hline 08 & 80 & 17 & 15 & 70 & 57 & 27 & 60 & 80 & 16 & 20 & 17 & 19 & 72 \\
\hline 73 & 19 & 20 & 21 & 21 & 15 & 20 & 16 & 20 & 20 & 23 & 21 & 23 & 19 \\
\hline 74 & 24 & 22 & 21 & 24 & 19 & 24 & 20 & 25 & 21 & 29 & 22 & 25 & 26 \\
\hline 75 & 21 & 22 & 23 & 21 & 16 & 21 & 17 & 22 & 22 & 26 & 25 & 26 & 20 \\
\hline 76 & 24 & 19 & 22 & 25 & 18 & 24 & 19 & 24 & 22 & 25 & 23 & 28 & 24 \\
\hline DS & 80 & 15 & 16 & 68 & 59 & 26 & 61 & 73 & 15 & 20 & 15 & 19 & - \\
\hline
\end{tabular}

Table B-141. Pennsylvania Site 4 Left, Short Wavelengths.

\begin{tabular}{|c|c|c|c|c|c|c|c|c|c|c|c|c|c|}
\hline $\begin{array}{c}\text { Correlation } \\
\text { Reference }\end{array}$ & 01 & 02 & 03 & 04 & 05 & 06 & 07 & 08 & 73 & 74 & 75 & 76 & DS \\
\hline 01 & 36 & 4 & 3 & 14 & 10 & 5 & 11 & 27 & 4 & 5 & 4 & 5 & 36 \\
\hline 02 & 4 & 11 & 7 & 5 & 4 & 8 & 5 & 5 & 8 & 8 & 7 & 8 & 5 \\
\hline 03 & 4 & 7 & 12 & 5 & 3 & 8 & 5 & 5 & 7 & 8 & 7 & 8 & 5 \\
\hline 04 & 22 & 4 & 4 & 19 & 11 & 6 & 10 & 24 & 4 & 5 & 4 & 5 & 32 \\
\hline 05 & 10 & 2 & 2 & 5 & 46 & 3 & 12 & 11 & 2 & 3 & 3 & 2 & 10 \\
\hline 06 & 7 & 6 & 5 & 8 & 5 & 9 & 6 & 7 & 5 & 5 & 6 & 6 & 7 \\
\hline 07 & 14 & 3 & 3 & 7 & 12 & 4 & 14 & 15 & 3 & 4 & 3 & 3 & 13 \\
\hline 08 & 29 & 3 & 3 & 12 & 14 & 5 & 11 & 32 & 3 & 5 & 4 & 4 & 24 \\
\hline 73 & 4 & 8 & 8 & 5 & 3 & 8 & 4 & 5 & 10 & 8 & 8 & 8 & 4 \\
\hline 74 & 6 & 7 & 7 & 6 & 4 & 8 & 5 & 6 & 6 & 12 & 7 & 9 & 6 \\
\hline 75 & 5 & 8 & 8 & 5 & 4 & 8 & 5 & 6 & 8 & 9 & 13 & 9 & 5 \\
\hline 76 & 5 & 7 & 7 & 6 & 3 & 8 & 5 & 5 & 7 & 9 & 7 & 13 & 5 \\
\hline DS & 26 & 3 & 3 & 11 & 14 & 4 & 8 & 23 & 2 & 4 & 3 & 3 & - \\
\hline
\end{tabular}


Table B-142. Pennsylvania Site 4 Right, IRI Filter.

\begin{tabular}{|c|c|c|c|c|c|c|c|c|c|c|c|c|c|}
\hline $\begin{array}{c}\text { Correlation } \\
\text { Reference }\end{array}$ & 01 & 02 & 03 & 04 & 05 & 06 & 07 & 08 & 73 & 74 & 75 & 76 & DS \\
\hline 01 & $\mathbf{9 7}$ & 65 & 56 & 73 & 89 & 72 & 85 & 91 & 62 & 60 & 74 & 77 & 88 \\
\hline 02 & 66 & 73 & 65 & 49 & 63 & 76 & 68 & 61 & 68 & 69 & 73 & 74 & 62 \\
\hline 03 & 57 & 67 & 62 & 43 & 56 & 70 & 58 & 54 & 63 & 64 & 67 & 67 & 55 \\
\hline 04 & 73 & 48 & 42 & 83 & 72 & 53 & 64 & 78 & 47 & 45 & 56 & 59 & 77 \\
\hline 05 & 89 & 61 & 54 & 73 & $\mathbf{9 6}$ & 69 & 88 & 85 & 59 & 56 & 70 & 74 & 87 \\
\hline 06 & 73 & 74 & 67 & 54 & 71 & 79 & 75 & 67 & 71 & 69 & 79 & 81 & 68 \\
\hline 07 & 85 & 65 & 56 & 64 & 88 & 73 & $\mathbf{9 4}$ & 77 & 62 & 60 & 73 & 77 & 78 \\
\hline 08 & 91 & 59 & 52 & 78 & 83 & 66 & 74 & $\mathbf{9 5}$ & 57 & 55 & 69 & 72 & 92 \\
\hline 73 & 63 & 67 & 60 & 48 & 62 & 72 & 64 & 59 & 62 & 63 & 71 & 73 & 60 \\
\hline 74 & 61 & 70 & 62 & 45 & 59 & 72 & 63 & 58 & 65 & 66 & 70 & 70 & 58 \\
\hline 75 & 75 & 70 & 62 & 57 & 72 & 78 & 75 & 71 & 69 & 66 & 79 & 82 & 71 \\
\hline 76 & 78 & 70 & 62 & 60 & 76 & 79 & 79 & 73 & 69 & 65 & 80 & 83 & 74 \\
\hline DS & 89 & 59 & 52 & 78 & 86 & 66 & 77 & 92 & 57 & 54 & 68 & 72 & - \\
\hline
\end{tabular}

Table B-143. Pennsylvania Site 4 Right, Long Wavelengths.

\begin{tabular}{|c|c|c|c|c|c|c|c|c|c|c|c|c|c|}
\hline $\begin{array}{c}\text { Correlation } \\
\text { Reference }\end{array}$ & 01 & 02 & 03 & 04 & 05 & 06 & 07 & 08 & 73 & 74 & 75 & 76 & DS \\
\hline 01 & 99 & 75 & 73 & 77 & 86 & 73 & 83 & 94 & 76 & 78 & 77 & 77 & 92 \\
\hline 02 & 75 & 95 & 92 & 61 & 79 & 93 & 81 & 72 & 94 & 93 & 94 & 94 & 70 \\
\hline 03 & 74 & 92 & 91 & 60 & 79 & 92 & 80 & 71 & 92 & 92 & 92 & 92 & 69 \\
\hline 04 & 78 & 61 & 60 & 78 & 70 & 60 & 67 & 81 & 63 & 65 & 64 & 65 & 80 \\
\hline 05 & 85 & 79 & 78 & 69 & 99 & 78 & 96 & 82 & 80 & 83 & 82 & 83 & 80 \\
\hline 06 & 73 & 93 & 92 & 59 & 79 & 93 & 80 & 70 & 92 & 92 & 93 & 93 & 69 \\
\hline 07 & 83 & 80 & 79 & 67 & 96 & 79 & 98 & 79 & 81 & 84 & 82 & 83 & 77 \\
\hline 08 & 95 & 72 & 71 & 80 & 83 & 70 & 79 & 95 & 73 & 76 & 75 & 75 & 93 \\
\hline 73 & 76 & 94 & 91 & 63 & 81 & 92 & 82 & 73 & 94 & 94 & 95 & 95 & 71 \\
\hline 74 & 78 & 93 & 91 & 64 & 83 & 92 & 84 & 75 & 94 & 96 & 96 & 96 & 72 \\
\hline 75 & 77 & 94 & 92 & 64 & 82 & 93 & 83 & 75 & 95 & 96 & 97 & 97 & 72 \\
\hline 76 & 77 & 93 & 92 & 64 & 83 & 93 & 84 & 75 & 95 & 96 & 97 & 97 & 72 \\
\hline DS & 92 & 69 & 69 & 80 & 81 & 68 & 77 & 92 & 71 & 73 & 72 & 73 & - \\
\hline
\end{tabular}


Table B-144. Pennsylvania Site 4 Right, Medium Wavelengths.

\begin{tabular}{|c|c|c|c|c|c|c|c|c|c|c|c|c|c|}
\hline $\begin{array}{c}\text { Correlation } \\
\text { Reference }\end{array}$ & 01 & 02 & 03 & 04 & 05 & 06 & 07 & 08 & 73 & 74 & 75 & 76 & DS \\
\hline 01 & 91 & 38 & 22 & 64 & 79 & 52 & 81 & 81 & 30 & 26 & 44 & 49 & 76 \\
\hline 02 & 42 & 41 & 26 & 30 & 38 & 44 & 45 & 39 & 34 & 31 & 40 & 41 & 37 \\
\hline 03 & 25 & 31 & 25 & 20 & 26 & 31 & 27 & 24 & 27 & 27 & 29 & 29 & 25 \\
\hline 04 & 64 & 26 & 17 & 73 & 72 & 40 & 58 & 69 & 24 & 19 & 35 & 41 & 63 \\
\hline 05 & 80 & 33 & 21 & 73 & 86 & 48 & 74 & 79 & 29 & 24 & 40 & 47 & 75 \\
\hline 06 & 57 & 38 & 24 & 44 & 55 & 49 & 60 & 53 & 32 & 27 & 44 & 49 & 53 \\
\hline 07 & 82 & 39 & 22 & 60 & 74 & 53 & 84 & 72 & 31 & 27 & 43 & 49 & 70 \\
\hline 08 & 81 & 33 & 20 & 69 & 78 & 47 & 71 & 85 & 28 & 24 & 41 & 45 & 74 \\
\hline 73 & 34 & 34 & 23 & 27 & 34 & 38 & 37 & 33 & 27 & 26 & 35 & 38 & 33 \\
\hline 74 & 31 & 36 & 26 & 22 & 29 & 34 & 33 & 30 & 30 & 32 & 33 & 32 & 28 \\
\hline 75 & 49 & 35 & 23 & 39 & 47 & 47 & 51 & 47 & 31 & 27 & 42 & 47 & 47 \\
\hline 76 & 54 & 34 & 22 & 46 & 54 & 48 & 56 & 52 & 31 & 25 & 43 & 48 & 53 \\
\hline DS & 77 & 31 & 19 & 69 & 79 & 46 & 67 & 79 & 27 & 22 & 39 & 45 & - \\
\hline
\end{tabular}

Table B-145. Pennsylvania Site 4 Right, Short Wavelengths.

\begin{tabular}{|c|c|c|c|c|c|c|c|c|c|c|c|c|c|}
\hline $\begin{array}{c}\text { Correlation } \\
\text { Reference }\end{array}$ & 01 & 02 & 03 & 04 & 05 & 06 & 07 & 08 & 73 & 74 & 75 & 76 & DS \\
\hline 01 & 54 & 12 & 4 & 14 & 27 & 15 & 40 & 37 & 7 & 5 & 10 & 11 & 34 \\
\hline 02 & 15 & 16 & 6 & 8 & 11 & 13 & 14 & 11 & 8 & 7 & 11 & 14 & 10 \\
\hline 03 & 5 & 10 & 11 & 5 & 6 & 9 & 5 & 4 & 9 & 9 & 11 & 10 & 5 \\
\hline 04 & 19 & 6 & 4 & 30 & 28 & 12 & 18 & 23 & 6 & 4 & 10 & 13 & 19 \\
\hline 05 & 28 & 7 & 4 & 19 & 40 & 13 & 27 & 31 & 6 & 4 & 9 & 11 & 27 \\
\hline 06 & 20 & 9 & 5 & 13 & 21 & 16 & 19 & 19 & 7 & 6 & 10 & 13 & 17 \\
\hline 07 & 40 & 9 & 3 & 11 & 27 & 12 & 41 & 35 & 6 & 5 & 8 & 9 & 21 \\
\hline 08 & 37 & 8 & 3 & 12 & 24 & 14 & 28 & 48 & 6 & 5 & 10 & 11 & 24 \\
\hline 73 & 8 & 9 & 6 & 7 & 9 & 11 & 8 & 7 & 9 & 7 & 12 & 12 & 8 \\
\hline 74 & 7 & 11 & 7 & 5 & 7 & 10 & 6 & 6 & 10 & 13 & 11 & 10 & 6 \\
\hline 75 & 11 & 8 & 5 & 11 & 14 & 12 & 10 & 12 & 9 & 6 & 17 & 15 & 11 \\
\hline 76 & 13 & 9 & 5 & 14 & 18 & 12 & 12 & 13 & 7 & 6 & 12 & 18 & 16 \\
\hline DS & 27 & 6 & 3 & 12 & 26 & 11 & 24 & 32 & 5 & 4 & 7 & 10 & - \\
\hline
\end{tabular}


Table B-146. Pennsylvania Site 5 Left, IRI Filter.

\begin{tabular}{|c|c|c|c|c|c|c|c|c|c|c|c|c|c|}
\hline $\begin{array}{c}\text { Correlation } \\
\text { Reference }\end{array}$ & 01 & 02 & 03 & 04 & 05 & 06 & 07 & 08 & 73 & 74 & 75 & 76 & DS \\
\hline 01 & $\mathbf{9 5}$ & 74 & 74 & 89 & 81 & 77 & 87 & 90 & 79 & 75 & 79 & 78 & 84 \\
\hline 02 & 77 & 83 & 80 & 73 & 74 & 83 & 80 & 77 & 85 & 85 & 83 & 84 & 77 \\
\hline 03 & 77 & 77 & 82 & 75 & 75 & 80 & 78 & 77 & 82 & 80 & 81 & 80 & 78 \\
\hline 04 & 89 & 69 & 72 & 91 & 78 & 72 & 82 & 86 & 75 & 73 & 75 & 73 & 84 \\
\hline 05 & 82 & 70 & 70 & 78 & $\mathbf{9 5}$ & 69 & 92 & 77 & 73 & 71 & 72 & 70 & 78 \\
\hline 06 & 79 & 82 & 83 & 75 & 74 & 86 & 79 & 78 & 86 & 85 & 85 & 86 & 79 \\
\hline 07 & 89 & 74 & 74 & 84 & 92 & 74 & $\mathbf{9 7}$ & 85 & 78 & 76 & 78 & 76 & 82 \\
\hline 08 & 91 & 73 & 72 & 87 & 77 & 74 & 86 & 92 & 78 & 76 & 78 & 76 & 85 \\
\hline 73 & 81 & 81 & 81 & 79 & 78 & 82 & 83 & 82 & 86 & 83 & 85 & 84 & 82 \\
\hline 74 & 81 & 82 & 81 & 78 & 75 & 83 & 81 & 81 & 86 & 87 & 86 & 85 & 81 \\
\hline 75 & 84 & 78 & 78 & 80 & 77 & 79 & 83 & 84 & 83 & 81 & 85 & 82 & 84 \\
\hline 76 & 81 & 81 & 81 & 77 & 75 & 83 & 81 & 81 & 86 & 84 & 86 & 87 & 81 \\
\hline DS & 89 & 72 & 73 & 88 & 81 & 73 & 87 & 87 & 77 & 75 & 78 & 75 & - \\
\hline
\end{tabular}

Table B-147. Pennsylvania Site 5 Left, Long Wavelengths.

\begin{tabular}{|c|c|c|c|c|c|c|c|c|c|c|c|c|c|}
\hline $\begin{array}{c}\text { Correlation } \\
\text { Reference }\end{array}$ & 01 & 02 & 03 & 04 & 05 & 06 & 07 & 08 & 73 & 74 & 75 & 76 & DS \\
\hline 01 & 98 & 79 & 79 & 91 & 80 & 78 & 84 & 96 & 82 & 82 & 83 & 81 & 96 \\
\hline 02 & 79 & 96 & 94 & 73 & 86 & 95 & 86 & 77 & 96 & 95 & 94 & 96 & 79 \\
\hline 03 & 79 & 93 & 95 & 73 & 86 & 92 & 86 & 79 & 94 & 94 & 94 & 94 & 79 \\
\hline 04 & 91 & 73 & 72 & 86 & 72 & 71 & 76 & 90 & 75 & 75 & 77 & 75 & 88 \\
\hline 05 & 80 & 85 & 86 & 72 & 99 & 85 & 96 & 75 & 88 & 89 & 89 & 88 & 80 \\
\hline 06 & 78 & 95 & 92 & 71 & 85 & 94 & 85 & 76 & 94 & 94 & 93 & 95 & 77 \\
\hline 07 & 85 & 85 & 86 & 76 & 96 & 85 & 99 & 81 & 88 & 89 & 89 & 88 & 84 \\
\hline 08 & 96 & 77 & 78 & 90 & 75 & 75 & 81 & 96 & 79 & 80 & 81 & 79 & 94 \\
\hline 73 & 82 & 95 & 94 & 75 & 88 & 94 & 88 & 80 & 97 & 97 & 96 & 97 & 81 \\
\hline 74 & 82 & 95 & 94 & 76 & 89 & 93 & 89 & 80 & 97 & 97 & 97 & 97 & 82 \\
\hline 75 & 83 & 94 & 94 & 77 & 89 & 93 & 89 & 81 & 96 & 97 & 97 & 96 & 83 \\
\hline 76 & 81 & 96 & 94 & 75 & 88 & 95 & 88 & 79 & 97 & 97 & 97 & 98 & 81 \\
\hline DS & 96 & 79 & 78 & 89 & 80 & 77 & 84 & 94 & 81 & 81 & 82 & 80 & - \\
\hline
\end{tabular}


Table B-148. Pennsylvania Site 5 Left, Medium Wavelengths.

\begin{tabular}{|c|c|c|c|c|c|c|c|c|c|c|c|c|c|}
\hline $\begin{array}{c}\text { Correlation } \\
\text { Reference }\end{array}$ & 01 & 02 & 03 & 04 & 05 & 06 & 07 & 08 & 73 & 74 & 75 & 76 & DS \\
\hline 01 & 91 & 68 & 67 & 83 & 75 & 75 & 82 & 84 & 74 & 69 & 72 & 68 & 75 \\
\hline 02 & 75 & 73 & 68 & 74 & 71 & 71 & 76 & 77 & 75 & 73 & 73 & 71 & 74 \\
\hline 03 & 73 & 64 & 70 & 76 & 70 & 67 & 72 & 71 & 70 & 64 & 66 & 65 & 70 \\
\hline 04 & 84 & 67 & 70 & 88 & 76 & 72 & 81 & 80 & 73 & 69 & 71 & 69 & 77 \\
\hline 05 & 75 & 61 & 60 & 72 & 92 & 61 & 88 & 70 & 66 & 61 & 62 & 61 & 68 \\
\hline 06 & 82 & 68 & 70 & 78 & 70 & 75 & 75 & 79 & 75 & 71 & 72 & 72 & 76 \\
\hline 07 & 81 & 65 & 63 & 76 & 88 & 65 & 94 & 78 & 71 & 66 & 67 & 65 & 70 \\
\hline 08 & 86 & 69 & 65 & 81 & 73 & 72 & 81 & 87 & 75 & 71 & 73 & 71 & 75 \\
\hline 73 & 81 & 68 & 69 & 79 & 75 & 71 & 80 & 80 & 77 & 69 & 73 & 71 & 77 \\
\hline 74 & 80 & 70 & 68 & 79 & 71 & 73 & 76 & 81 & 75 & 78 & 74 & 72 & 77 \\
\hline 75 & 82 & 66 & 65 & 78 & 72 & 68 & 78 & 79 & 72 & 67 & 74 & 70 & 75 \\
\hline 76 & 82 & 68 & 68 & 78 & 70 & 73 & 76 & 81 & 76 & 71 & 75 & 77 & 78 \\
\hline DS & 82 & 65 & 65 & 81 & 74 & 68 & 80 & 79 & 72 & 68 & 71 & 67 & - \\
\hline
\end{tabular}

Table B-149. Pennsylvania Site 5 Left, Short Wavelengths.

\begin{tabular}{|c|c|c|c|c|c|c|c|c|c|c|c|c|c|}
\hline $\begin{array}{c}\text { Correlation } \\
\text { Reference }\end{array}$ & 01 & 02 & 03 & 04 & 05 & 06 & 07 & 08 & 73 & 74 & 75 & 76 & DS \\
\hline 01 & 65 & 43 & 34 & 34 & 26 & 48 & 34 & 49 & 43 & 45 & 49 & 39 & 45 \\
\hline 02 & 27 & 41 & 29 & 22 & 40 & 28 & 38 & 33 & 41 & 36 & 37 & 31 & 34 \\
\hline 03 & 22 & 27 & 39 & 22 & 32 & 30 & 25 & 25 & 40 & 28 & 32 & 32 & 30 \\
\hline 04 & 41 & 45 & 42 & 53 & 37 & 44 & 38 & 41 & 47 & 45 & 44 & 41 & 50 \\
\hline 05 & 25 & 25 & 23 & 21 & 70 & 24 & 48 & 23 & 33 & 26 & 26 & 25 & 32 \\
\hline 06 & 35 & 29 & 33 & 25 & 39 & 47 & 35 & 39 & 47 & 37 & 34 & 39 & 39 \\
\hline 07 & 32 & 27 & 23 & 22 & 48 & 27 & 58 & 36 & 39 & 34 & 30 & 31 & 32 \\
\hline 08 & 48 & 40 & 31 & 26 & 30 & 40 & 43 & 62 & 48 & 46 & 43 & 41 & 37 \\
\hline 73 & 26 & 29 & 31 & 20 & 40 & 34 & 32 & 30 & 50 & 31 & 35 & 37 & 37 \\
\hline 74 & 30 & 33 & 26 & 22 & 34 & 32 & 34 & 35 & 39 & 53 & 35 & 34 & 38 \\
\hline 75 & 33 & 33 & 30 & 23 & 35 & 30 & 35 & 38 & 43 & 35 & 50 & 40 & 43 \\
\hline 76 & 35 & 30 & 33 & 24 & 36 & 37 & 36 & 39 & 49 & 37 & 43 & 49 & 44 \\
\hline DS & 32 & 32 & 30 & 24 & 42 & 36 & 41 & 37 & 48 & 40 & 42 & 38 & - \\
\hline
\end{tabular}


Table B-150. Pennsylvania Site 5 Right, IRI Filter.

\begin{tabular}{|c|c|c|c|c|c|c|c|c|c|c|c|c|c|}
\hline $\begin{array}{c}\text { Correlation } \\
\text { Reference }\end{array}$ & 01 & 02 & 03 & 04 & 05 & 06 & 07 & 08 & 73 & 74 & 75 & 76 & DS \\
\hline 01 & $\mathbf{9 7}$ & 77 & 73 & 90 & 86 & 76 & 88 & 93 & 79 & 75 & 82 & 80 & 89 \\
\hline 02 & 80 & 86 & 81 & 73 & 75 & 85 & 84 & 77 & 88 & 84 & 86 & 87 & 75 \\
\hline 03 & 76 & 81 & 79 & 72 & 76 & 80 & 82 & 73 & 83 & 78 & 82 & 82 & 72 \\
\hline 04 & 89 & 70 & 69 & 88 & 84 & 70 & 82 & 87 & 73 & 68 & 74 & 74 & 84 \\
\hline 05 & 87 & 71 & 72 & 86 & $\mathbf{9 6}$ & 73 & 90 & 82 & 75 & 70 & 75 & 75 & 83 \\
\hline 06 & 78 & 86 & 82 & 72 & 76 & 88 & 83 & 75 & 87 & 84 & 86 & 88 & 73 \\
\hline 07 & 89 & 80 & 78 & 84 & 90 & 79 & $\mathbf{9 8}$ & 85 & 83 & 78 & 84 & 83 & 83 \\
\hline 08 & $\mathbf{9 4}$ & 73 & 69 & 88 & 81 & 72 & 83 & $\mathbf{9 5}$ & 76 & 72 & 78 & 77 & 88 \\
\hline 73 & 83 & 85 & 82 & 76 & 78 & 83 & 87 & 80 & 89 & 83 & 88 & 87 & 78 \\
\hline 74 & 78 & 84 & 80 & 72 & 74 & 84 & 82 & 76 & 86 & 82 & 85 & 86 & 73 \\
\hline 75 & 85 & 82 & 78 & 78 & 79 & 81 & 88 & 82 & 86 & 80 & 88 & 86 & 79 \\
\hline 76 & 83 & 84 & 81 & 77 & 79 & 84 & 87 & 80 & 87 & 83 & 89 & 89 & 78 \\
\hline DS & 90 & 72 & 68 & 86 & 81 & 70 & 82 & 87 & 74 & 69 & 76 & 74 & - \\
\hline
\end{tabular}

Table B-151. Pennsylvania Site 5 Right, Long Wavelengths.

\begin{tabular}{|c|c|c|c|c|c|c|c|c|c|c|c|c|c|}
\hline $\begin{array}{c}\text { Correlation } \\
\text { Reference }\end{array}$ & 01 & 02 & 03 & 04 & 05 & 06 & 07 & 08 & 73 & 74 & 75 & 76 & DS \\
\hline 01 & 98 & 82 & 84 & 89 & 87 & 78 & 87 & 98 & 83 & 83 & 84 & 83 & 91 \\
\hline 02 & 83 & 97 & 95 & 78 & 82 & 93 & 88 & 81 & 97 & 97 & 96 & 97 & 76 \\
\hline 03 & 85 & 94 & 96 & 80 & 86 & 90 & 90 & 83 & 95 & 95 & 96 & 95 & 78 \\
\hline 04 & 89 & 78 & 79 & 84 & 79 & 73 & 81 & 89 & 79 & 78 & 80 & 78 & 84 \\
\hline 05 & 88 & 82 & 85 & 80 & 99 & 79 & 95 & 83 & 83 & 82 & 84 & 83 & 82 \\
\hline 06 & 78 & 94 & 91 & 73 & 79 & 94 & 84 & 76 & 93 & 94 & 92 & 93 & 72 \\
\hline 07 & 88 & 87 & 90 & 81 & 95 & 84 & 99 & 84 & 88 & 88 & 89 & 88 & 82 \\
\hline 08 & 98 & 80 & 82 & 89 & 83 & 75 & 84 & 98 & 81 & 81 & 83 & 81 & 90 \\
\hline 73 & 84 & 97 & 95 & 79 & 83 & 93 & 89 & 82 & 97 & 97 & 97 & 97 & 77 \\
\hline 74 & 83 & 97 & 95 & 79 & 83 & 93 & 88 & 81 & 97 & 97 & 97 & 97 & 77 \\
\hline 75 & 85 & 96 & 96 & 80 & 84 & 91 & 89 & 83 & 97 & 96 & 98 & 97 & 78 \\
\hline 76 & 84 & 97 & 95 & 79 & 83 & 93 & 89 & 82 & 97 & 97 & 97 & 97 & 77 \\
\hline DS & 91 & 76 & 78 & 84 & 81 & 71 & 81 & 90 & 77 & 76 & 78 & 77 & - \\
\hline
\end{tabular}


Table B-152. Pennsylvania Site 5 Right, Medium Wavelengths.

\begin{tabular}{|c|c|c|c|c|c|c|c|c|c|c|c|c|c|}
\hline $\begin{array}{c}\text { Correlation } \\
\text { Reference }\end{array}$ & 01 & 02 & 03 & 04 & 05 & 06 & 07 & 08 & 73 & 74 & 75 & 76 & DS \\
\hline 01 & 95 & 74 & 64 & 86 & 86 & 75 & 89 & 90 & 76 & 69 & 79 & 76 & 79 \\
\hline 02 & 79 & 77 & 68 & 72 & 75 & 76 & 84 & 76 & 80 & 71 & 77 & 77 & 75 \\
\hline 03 & 70 & 69 & 66 & 69 & 72 & 69 & 76 & 67 & 72 & 65 & 69 & 70 & 68 \\
\hline 04 & 87 & 66 & 62 & 86 & 87 & 70 & 83 & 83 & 70 & 63 & 72 & 72 & 74 \\
\hline 05 & 87 & 67 & 64 & 88 & 92 & 70 & 88 & 83 & 71 & 64 & 72 & 72 & 76 \\
\hline 06 & 80 & 75 & 67 & 75 & 78 & 79 & 84 & 76 & 78 & 72 & 78 & 79 & 76 \\
\hline 07 & 91 & 75 & 68 & 86 & 88 & 76 & 96 & 87 & 80 & 72 & 81 & 80 & 84 \\
\hline 08 & 91 & 69 & 60 & 83 & 81 & 69 & 85 & 91 & 72 & 66 & 75 & 73 & 75 \\
\hline 73 & 83 & 76 & 67 & 77 & 79 & 73 & 87 & 80 & 80 & 70 & 79 & 77 & 79 \\
\hline 74 & 76 & 73 & 65 & 69 & 71 & 74 & 80 & 74 & 76 & 70 & 75 & 76 & 72 \\
\hline 75 & 86 & 71 & 62 & 78 & 79 & 70 & 85 & 83 & 75 & 67 & 77 & 75 & 78 \\
\hline 76 & 84 & 73 & 65 & 77 & 79 & 75 & 87 & 81 & 77 & 71 & 79 & 80 & 79 \\
\hline DS & 87 & 69 & 60 & 80 & 81 & 68 & 84 & 83 & 72 & 64 & 75 & 72 & - \\
\hline
\end{tabular}

Table B-153. Pennsylvania Site 5 Right, Short Wavelengths.

\begin{tabular}{|c|c|c|c|c|c|c|c|c|c|c|c|c|c|}
\hline $\begin{array}{c}\text { Correlation } \\
\text { Reference }\end{array}$ & 01 & 02 & 03 & 04 & 05 & 06 & 07 & 08 & 73 & 74 & 75 & 76 & DS \\
\hline 01 & 68 & 43 & 20 & 27 & 38 & 41 & 56 & 52 & 46 & 36 & 48 & 42 & 29 \\
\hline 02 & 30 & 41 & 21 & 16 & 31 & 27 & 41 & 33 & 41 & 29 & 37 & 31 & 23 \\
\hline 03 & 17 & 28 & 25 & 17 & 28 & 22 & 24 & 15 & 32 & 23 & 25 & 24 & 14 \\
\hline 04 & 33 & 30 & 22 & 44 & 52 & 32 & 46 & 25 & 38 & 28 & 34 & 35 & 20 \\
\hline 05 & 34 & 29 & 21 & 30 & 62 & 33 & 54 & 29 & 36 & 26 & 34 & 36 & 21 \\
\hline 06 & 33 & 30 & 19 & 20 & 37 & 38 & 42 & 32 & 34 & 30 & 32 & 35 & 16 \\
\hline 07 & 50 & 34 & 19 & 26 & 54 & 32 & 74 & 49 & 42 & 33 & 40 & 42 & 22 \\
\hline 08 & 50 & 35 & 15 & 15 & 27 & 30 & 50 & 63 & 39 & 34 & 41 & 41 & 23 \\
\hline 73 & 28 & 34 & 20 & 17 & 31 & 25 & 39 & 30 & 43 & 27 & 38 & 33 & 22 \\
\hline 74 & 28 & 29 & 17 & 16 & 28 & 27 & 37 & 31 & 33 & 33 & 35 & 34 & 20 \\
\hline 75 & 32 & 30 & 15 & 14 & 28 & 23 & 39 & 35 & 37 & 28 & 43 & 37 & 27 \\
\hline 76 & 34 & 26 & 15 & 16 & 31 & 27 & 42 & 35 & 34 & 30 & 39 & 45 & 24 \\
\hline DS & 41 & 37 & 16 & 18 & 33 & 25 & 40 & 38 & 37 & 30 & 45 & 41 & - \\
\hline
\end{tabular}


Table B-154. Pennsylvania Site 6 Left, IRI Filter.

\begin{tabular}{|c|c|c|c|c|c|c|c|c|c|c|c|c|c|}
\hline $\begin{array}{c}\text { Correlation } \\
\text { Reference }\end{array}$ & 01 & 02 & 03 & 04 & 05 & 06 & 07 & 08 & 73 & 74 & 75 & 76 & DS \\
\hline 01 & $\mathbf{9 4}$ & 85 & 78 & 87 & 91 & 76 & 93 & 88 & 84 & 80 & 86 & 83 & 88 \\
\hline 02 & 90 & 86 & 80 & 86 & 89 & 77 & 89 & 87 & 86 & 84 & 85 & 84 & 83 \\
\hline 03 & 83 & 78 & 89 & 84 & 85 & 71 & 88 & 75 & 82 & 78 & 80 & 80 & 84 \\
\hline 04 & 88 & 87 & 85 & $\mathbf{9 5}$ & 88 & 80 & 89 & 89 & 86 & 84 & 80 & 89 & 84 \\
\hline 05 & 93 & 83 & 81 & 86 & $\mathbf{9 6}$ & 75 & 92 & 86 & 86 & 81 & 86 & 82 & 85 \\
\hline 06 & 82 & 81 & 77 & 83 & 82 & 75 & 82 & 83 & 79 & 81 & 78 & 80 & 76 \\
\hline 07 & $\mathbf{9 4}$ & 82 & 81 & 86 & 92 & 75 & $\mathbf{9 6}$ & 86 & 85 & 81 & 87 & 82 & 89 \\
\hline 08 & 90 & 85 & 75 & 87 & 88 & 79 & 87 & $\mathbf{9 5}$ & 84 & 86 & 85 & 85 & 79 \\
\hline 73 & 90 & 82 & 80 & 84 & 89 & 75 & 90 & 83 & 86 & 80 & 85 & 82 & 86 \\
\hline 74 & 89 & 84 & 80 & 86 & 88 & 77 & 90 & 87 & 85 & 89 & 86 & 84 & 84 \\
\hline 75 & 89 & 79 & 74 & 80 & 85 & 73 & 88 & 82 & 82 & 79 & 85 & 79 & 86 \\
\hline 76 & 89 & 84 & 82 & 87 & 88 & 77 & 91 & 86 & 85 & 84 & 86 & 88 & 85 \\
\hline DS & 86 & 75 & 75 & 80 & 84 & 69 & 88 & 76 & 77 & 74 & 79 & 76 & - \\
\hline
\end{tabular}

Table B-155. Pennsylvania Site 6 Left, Long Wavelengths.

\begin{tabular}{|c|c|c|c|c|c|c|c|c|c|c|c|c|c|}
\hline $\begin{array}{c}\text { Correlation } \\
\text { Reference }\end{array}$ & 01 & 02 & 03 & 04 & 05 & 06 & 07 & 08 & 73 & 74 & 75 & 76 & DS \\
\hline 01 & 98 & 82 & 86 & 78 & 85 & 82 & 90 & 96 & 85 & 85 & 88 & 87 & 93 \\
\hline 02 & 82 & 94 & 89 & 81 & 90 & 91 & 85 & 79 & 94 & 93 & 90 & 91 & 79 \\
\hline 03 & 88 & 88 & 93 & 80 & 91 & 88 & 91 & 84 & 91 & 91 & 92 & 93 & 84 \\
\hline 04 & 78 & 80 & 80 & 75 & 80 & 80 & 77 & 76 & 82 & 81 & 80 & 81 & 75 \\
\hline 05 & 85 & 88 & 91 & 80 & 99 & 86 & 94 & 80 & 91 & 91 & 91 & 92 & 83 \\
\hline 06 & 82 & 91 & 89 & 80 & 88 & 89 & 85 & 80 & 92 & 92 & 89 & 90 & 79 \\
\hline 07 & 90 & 83 & 89 & 77 & 93 & 83 & 99 & 86 & 87 & 87 & 89 & 90 & 88 \\
\hline 08 & 96 & 78 & 83 & 76 & 80 & 79 & 86 & 98 & 81 & 82 & 85 & 85 & 94 \\
\hline 73 & 86 & 93 & 93 & 82 & 92 & 91 & 88 & 83 & 95 & 94 & 93 & 94 & 82 \\
\hline 74 & 86 & 91 & 92 & 82 & 92 & 91 & 89 & 83 & 94 & 94 & 93 & 94 & 82 \\
\hline 75 & 90 & 88 & 93 & 80 & 91 & 88 & 91 & 87 & 92 & 92 & 94 & 94 & 85 \\
\hline 76 & 89 & 89 & 94 & 82 & 92 & 89 & 91 & 86 & 93 & 93 & 94 & 95 & 85 \\
\hline DS & 94 & 77 & 83 & 74 & 82 & 78 & 88 & 93 & 81 & 81 & 83 & 83 & - \\
\hline
\end{tabular}


Table B-156. Pennsylvania Site 6 Left, Medium Wavelengths.

\begin{tabular}{|c|c|c|c|c|c|c|c|c|c|c|c|c|c|}
\hline $\begin{array}{c}\text { Correlation } \\
\text { Reference }\end{array}$ & 01 & 02 & 03 & 04 & 05 & 06 & 07 & 08 & 73 & 74 & 75 & 76 & DS \\
\hline 01 & 94 & 85 & 75 & 87 & 92 & 74 & 94 & 87 & 83 & 79 & 85 & 83 & 85 \\
\hline 02 & 90 & 84 & 78 & 84 & 88 & 73 & 89 & 84 & 84 & 81 & 84 & 81 & 84 \\
\hline 03 & 81 & 73 & 87 & 81 & 82 & 66 & 87 & 70 & 78 & 74 & 77 & 76 & 84 \\
\hline 04 & 88 & 86 & 82 & 95 & 89 & 77 & 89 & 88 & 86 & 82 & 80 & 88 & 84 \\
\hline 05 & 94 & 81 & 80 & 86 & 95 & 73 & 91 & 84 & 85 & 81 & 85 & 79 & 85 \\
\hline 06 & 82 & 78 & 74 & 80 & 81 & 71 & 82 & 79 & 77 & 77 & 76 & 76 & 75 \\
\hline 07 & 93 & 79 & 79 & 85 & 91 & 72 & 96 & 83 & 82 & 80 & 84 & 79 & 88 \\
\hline 08 & 90 & 84 & 73 & 86 & 87 & 77 & 86 & 95 & 83 & 85 & 83 & 83 & 77 \\
\hline 73 & 89 & 79 & 78 & 83 & 88 & 72 & 90 & 80 & 85 & 78 & 83 & 79 & 86 \\
\hline 74 & 90 & 80 & 77 & 83 & 87 & 72 & 90 & 84 & 81 & 87 & 84 & 80 & 83 \\
\hline 75 & 86 & 76 & 72 & 78 & 83 & 69 & 86 & 79 & 78 & 76 & 83 & 76 & 84 \\
\hline 76 & 89 & 80 & 80 & 85 & 87 & 73 & 90 & 83 & 83 & 81 & 84 & 86 & 85 \\
\hline DS & 83 & 73 & 73 & 78 & 82 & 66 & 86 & 74 & 75 & 72 & 77 & 74 & - \\
\hline
\end{tabular}

Table B-157. Pennsylvania Site 6 Left, Short Wavelengths.

\begin{tabular}{|c|c|c|c|c|c|c|c|c|c|c|c|c|c|}
\hline $\begin{array}{c}\text { Correlation } \\
\text { Reference }\end{array}$ & 01 & 02 & 03 & 04 & 05 & 06 & 07 & 08 & 73 & 74 & 75 & 76 & DS \\
\hline 01 & 81 & 64 & 33 & 52 & 75 & 47 & 70 & 67 & 60 & 57 & 66 & 64 & 63 \\
\hline 02 & 43 & 60 & 36 & 29 & 49 & 38 & 45 & 43 & 53 & 50 & 52 & 48 & 41 \\
\hline 03 & 25 & 33 & 64 & 24 & 34 & 27 & 34 & 21 & 43 & 41 & 38 & 40 & 30 \\
\hline 04 & 65 & 62 & 51 & 68 & 71 & 44 & 69 & 50 & 61 & 55 & 63 & 68 & 80 \\
\hline 05 & 65 & 51 & 45 & 40 & 85 & 45 & 72 & 54 & 58 & 56 & 54 & 50 & 48 \\
\hline 06 & 40 & 46 & 35 & 29 & 45 & 42 & 42 & 39 & 49 & 45 & 50 & 42 & 40 \\
\hline 07 & 58 & 49 & 47 & 41 & 72 & 44 & 74 & 50 & 58 & 55 & 54 & 51 & 43 \\
\hline 08 & 60 & 53 & 31 & 30 & 55 & 45 & 50 & 78 & 51 & 61 & 60 & 52 & 41 \\
\hline 73 & 39 & 47 & 40 & 30 & 47 & 39 & 44 & 36 & 64 & 44 & 52 & 48 & 40 \\
\hline 74 & 40 & 44 & 40 & 27 & 44 & 35 & 43 & 43 & 46 & 68 & 50 & 46 & 34 \\
\hline 75 & 41 & 44 & 33 & 26 & 46 & 39 & 43 & 42 & 50 & 47 & 62 & 47 & 44 \\
\hline 76 & 41 & 46 & 40 & 30 & 48 & 36 & 46 & 40 & 52 & 48 & 53 & 63 & 45 \\
\hline DS & 40 & 42 & 36 & 34 & 52 & 37 & 51 & 34 & 47 & 39 & 50 & 48 & - \\
\hline
\end{tabular}


Table B-158. Pennsylvania Site 6 Right, IRI Filter.

\begin{tabular}{|c|c|c|c|c|c|c|c|c|c|c|c|c|c|}
\hline $\begin{array}{c}\text { Correlation } \\
\text { Reference }\end{array}$ & 01 & 02 & 03 & 04 & 05 & 06 & 07 & 08 & 73 & 74 & 75 & 76 & DS \\
\hline 01 & $\mathbf{9 5}$ & 87 & 79 & 77 & 84 & 82 & 88 & 89 & 86 & 81 & 86 & 85 & 76 \\
\hline 02 & 90 & 88 & 81 & 77 & 85 & 83 & 88 & 88 & 87 & 84 & 87 & 85 & 74 \\
\hline 03 & 83 & 79 & 85 & 75 & 82 & 78 & 84 & 77 & 80 & 78 & 80 & 81 & 74 \\
\hline 04 & 78 & 77 & 75 & 79 & 86 & 81 & 87 & 81 & 77 & 80 & 76 & 79 & 66 \\
\hline 05 & 86 & 88 & 85 & 86 & $\mathbf{9 6}$ & 89 & $\mathbf{9 5}$ & 89 & 88 & 89 & 87 & 90 & 75 \\
\hline 06 & 87 & 88 & 83 & 82 & 89 & 89 & 92 & 90 & 88 & 87 & 87 & 89 & 72 \\
\hline 07 & 89 & 89 & 85 & 85 & $\mathbf{9 5}$ & 88 & $\mathbf{9 8}$ & 92 & 89 & 88 & 90 & 90 & 76 \\
\hline 08 & 90 & 86 & 77 & 80 & 88 & 85 & 91 & $\mathbf{9 6}$ & 86 & 85 & 89 & 87 & 72 \\
\hline 73 & 90 & 85 & 81 & 77 & 84 & 83 & 87 & 86 & 88 & 81 & 87 & 85 & 75 \\
\hline 74 & 89 & 87 & 81 & 80 & 87 & 85 & 90 & 90 & 85 & 90 & 88 & 87 & 75 \\
\hline 75 & 91 & 82 & 78 & 74 & 81 & 81 & 84 & 85 & 83 & 80 & 87 & 82 & 77 \\
\hline 76 & 90 & 85 & 83 & 79 & 87 & 84 & 89 & 89 & 86 & 84 & 87 & 89 & 77 \\
\hline DS & 76 & 68 & 67 & 64 & 72 & 67 & 74 & 71 & 68 & 68 & 70 & 70 & - \\
\hline
\end{tabular}

Table B-159. Pennsylvania Site 6 Right, Long Wavelengths.

\begin{tabular}{|c|c|c|c|c|c|c|c|c|c|c|c|c|c|}
\hline $\begin{array}{c}\text { Correlation } \\
\text { Reference }\end{array}$ & 01 & 02 & 03 & 04 & 05 & 06 & 07 & 08 & 73 & 74 & 75 & 76 & DS \\
\hline 01 & 98 & 86 & 87 & 77 & 91 & 84 & 88 & 95 & 86 & 85 & 90 & 88 & 79 \\
\hline 02 & 86 & 96 & 92 & 84 & 88 & 93 & 91 & 83 & 96 & 96 & 93 & 94 & 70 \\
\hline 03 & 89 & 91 & 92 & 81 & 91 & 89 & 91 & 85 & 92 & 92 & 93 & 93 & 73 \\
\hline 04 & 77 & 84 & 82 & 81 & 79 & 83 & 81 & 75 & 84 & 84 & 81 & 83 & 64 \\
\hline 05 & 91 & 87 & 90 & 79 & 99 & 85 & 96 & 89 & 88 & 88 & 91 & 91 & 77 \\
\hline 06 & 84 & 93 & 90 & 84 & 86 & 92 & 89 & 82 & 93 & 93 & 90 & 92 & 68 \\
\hline 07 & 88 & 89 & 91 & 81 & 96 & 87 & 99 & 85 & 91 & 90 & 93 & 93 & 74 \\
\hline 08 & 95 & 82 & 84 & 75 & 88 & 80 & 85 & 98 & 83 & 83 & 87 & 86 & 77 \\
\hline 73 & 87 & 95 & 93 & 84 & 89 & 92 & 92 & 84 & 95 & 95 & 94 & 95 & 71 \\
\hline 74 & 87 & 95 & 93 & 85 & 89 & 93 & 92 & 84 & 95 & 96 & 93 & 95 & 71 \\
\hline 75 & 91 & 91 & 92 & 80 & 92 & 90 & 93 & 88 & 93 & 92 & 95 & 94 & 74 \\
\hline 76 & 90 & 93 & 93 & 83 & 92 & 91 & 93 & 87 & 94 & 94 & 95 & 95 & 74 \\
\hline DS & 80 & 69 & 71 & 64 & 76 & 67 & 73 & 77 & 70 & 69 & 73 & 73 & - \\
\hline
\end{tabular}


Table B-160. Pennsylvania Site 6 Right, Medium Wavelengths.

\begin{tabular}{|c|c|c|c|c|c|c|c|c|c|c|c|c|c|}
\hline $\begin{array}{c}\text { Correlation } \\
\text { Reference }\end{array}$ & 01 & 02 & 03 & 04 & 05 & 06 & 07 & 08 & 73 & 74 & 75 & 76 & DS \\
\hline 01 & 94 & 86 & 76 & 78 & 85 & 81 & 89 & 88 & 86 & 81 & 84 & 83 & 75 \\
\hline 02 & 90 & 87 & 77 & 76 & 83 & 81 & 87 & 86 & 83 & 82 & 84 & 82 & 76 \\
\hline 03 & 81 & 75 & 83 & 74 & 79 & 74 & 81 & 73 & 76 & 74 & 77 & 77 & 75 \\
\hline 04 & 80 & 77 & 75 & 81 & 86 & 81 & 88 & 82 & 77 & 80 & 76 & 79 & 67 \\
\hline 05 & 87 & 86 & 83 & 86 & 95 & 87 & 95 & 90 & 86 & 87 & 87 & 88 & 77 \\
\hline 06 & 89 & 85 & 80 & 81 & 87 & 87 & 91 & 89 & 86 & 84 & 86 & 86 & 74 \\
\hline 07 & 91 & 86 & 83 & 85 & 95 & 87 & 98 & 93 & 87 & 87 & 89 & 88 & 77 \\
\hline 08 & 90 & 85 & 74 & 80 & 87 & 85 & 91 & 96 & 85 & 84 & 87 & 86 & 72 \\
\hline 73 & 90 & 83 & 78 & 77 & 83 & 81 & 86 & 84 & 86 & 78 & 85 & 82 & 76 \\
\hline 74 & 90 & 83 & 78 & 78 & 85 & 81 & 88 & 88 & 81 & 88 & 85 & 83 & 77 \\
\hline 75 & 88 & 78 & 74 & 73 & 80 & 78 & 83 & 83 & 80 & 78 & 85 & 79 & 77 \\
\hline 76 & 90 & 82 & 79 & 79 & 85 & 81 & 88 & 86 & 83 & 81 & 85 & 87 & 79 \\
\hline DS & 75 & 67 & 67 & 65 & 72 & 67 & 74 & 70 & 66 & 68 & 69 & 69 & - \\
\hline
\end{tabular}

Table B-161. Pennsylvania Site 6 Right, Short Wavelengths.

\begin{tabular}{|c|c|c|c|c|c|c|c|c|c|c|c|c|c|}
\hline $\begin{array}{c}\text { Correlation } \\
\text { Reference }\end{array}$ & 01 & 02 & 03 & 04 & 05 & 06 & 07 & 08 & 73 & 74 & 75 & 76 & DS \\
\hline 01 & 81 & 68 & 37 & 48 & 64 & 60 & 66 & 57 & 69 & 55 & 62 & 65 & 47 \\
\hline 02 & 42 & 64 & 32 & 28 & 41 & 44 & 49 & 46 & 52 & 51 & 48 & 49 & 36 \\
\hline 03 & 23 & 31 & 54 & 21 & 29 & 29 & 33 & 21 & 36 & 38 & 36 & 37 & 31 \\
\hline 04 & 58 & 49 & 45 & 60 & 63 & 55 & 71 & 44 & 52 & 58 & 57 & 56 & 47 \\
\hline 05 & 64 & 49 & 44 & 46 & 78 & 52 & 72 & 54 & 53 & 61 & 60 & 59 & 60 \\
\hline 06 & 43 & 48 & 34 & 31 & 45 & 59 & 54 & 48 & 55 & 49 & 59 & 53 & 38 \\
\hline 07 & 61 & 48 & 40 & 42 & 72 & 52 & 88 & 61 & 54 & 58 & 60 & 58 & 51 \\
\hline 08 & 53 & 50 & 27 & 26 & 42 & 49 & 54 & 83 & 54 & 55 & 55 & 57 & 34 \\
\hline 73 & 42 & 50 & 34 & 29 & 42 & 47 & 50 & 42 & 67 & 43 & 54 & 52 & 33 \\
\hline 74 & 39 & 44 & 33 & 24 & 37 & 38 & 45 & 47 & 40 & 66 & 48 & 46 & 34 \\
\hline 75 & 39 & 41 & 30 & 25 & 38 & 46 & 47 & 44 & 48 & 47 & 62 & 48 & 35 \\
\hline 76 & 40 & 43 & 33 & 28 & 42 & 42 & 49 & 44 & 50 & 48 & 51 & 64 & 38 \\
\hline DS & 31 & 33 & 31 & 25 & 39 & 33 & 45 & 32 & 34 & 39 & 37 & 40 & - \\
\hline
\end{tabular}


Table B-162. Pennsylvania Site 7 Left, IRI Filter.

\begin{tabular}{|c|c|c|c|c|c|c|c|c|c|c|c|c|c|}
\hline $\begin{array}{c}\text { Correlation } \\
\text { Reference }\end{array}$ & 01 & 02 & 03 & 04 & 05 & 06 & 07 & 08 & 73 & 74 & 75 & 76 & DS \\
\hline 01 & $\mathbf{9 4}$ & 84 & 80 & 89 & 84 & 73 & 89 & 89 & 85 & 86 & 84 & 85 & 75 \\
\hline 02 & 89 & 84 & 80 & 86 & 83 & 71 & 86 & 83 & 83 & 84 & 83 & 83 & 73 \\
\hline 03 & 80 & 73 & 89 & 81 & 74 & 63 & 80 & 75 & 76 & 76 & 75 & 79 & 77 \\
\hline 04 & 90 & 81 & 83 & 91 & 82 & 72 & 87 & 84 & 84 & 84 & 81 & 85 & 73 \\
\hline 05 & 86 & 87 & 79 & 85 & $\mathbf{9 5}$ & 70 & 83 & 76 & 83 & 81 & 82 & 80 & 69 \\
\hline 06 & 75 & 68 & 68 & 73 & 67 & 60 & 72 & 73 & 70 & 72 & 70 & 72 & 65 \\
\hline 07 & 90 & 80 & 82 & 87 & 83 & 70 & 87 & 85 & 82 & 83 & 83 & 83 & 75 \\
\hline 08 & 89 & 76 & 75 & 82 & 75 & 68 & 83 & 92 & 78 & 81 & 82 & 81 & 76 \\
\hline 73 & 87 & 78 & 82 & 85 & 78 & 70 & 85 & 84 & 82 & 82 & 82 & 82 & 76 \\
\hline 74 & 87 & 77 & 80 & 84 & 76 & 69 & 84 & 86 & 80 & 84 & 81 & 82 & 78 \\
\hline 75 & 87 & 77 & 78 & 82 & 76 & 67 & 83 & 85 & 79 & 80 & 81 & 80 & 77 \\
\hline 76 & 85 & 76 & 81 & 84 & 75 & 68 & 82 & 84 & 79 & 80 & 79 & 84 & 77 \\
\hline DS & 75 & 66 & 70 & 73 & 67 & 59 & 73 & 74 & 69 & 70 & 70 & 70 & - \\
\hline
\end{tabular}

Table B-163. Pennsylvania Site 7 Left, Long Wavelengths.

\begin{tabular}{|c|c|c|c|c|c|c|c|c|c|c|c|c|c|}
\hline $\begin{array}{c}\text { Correlation } \\
\text { Reference }\end{array}$ & 01 & 02 & 03 & 04 & 05 & 06 & 07 & 08 & 73 & 74 & 75 & 76 & DS \\
\hline 01 & 97 & 83 & 87 & 66 & 85 & 77 & 92 & 96 & 83 & 83 & 87 & 83 & 84 \\
\hline 02 & 84 & 92 & 91 & 71 & 92 & 86 & 86 & 80 & 92 & 92 & 91 & 91 & 72 \\
\hline 03 & 88 & 90 & 94 & 69 & 91 & 84 & 90 & 85 & 91 & 90 & 92 & 90 & 76 \\
\hline 04 & 66 & 70 & 69 & 58 & 70 & 68 & 67 & 65 & 71 & 71 & 68 & 72 & 58 \\
\hline 05 & 85 & 91 & 91 & 70 & 99 & 83 & 91 & 80 & 91 & 90 & 91 & 89 & 72 \\
\hline 06 & 78 & 86 & 85 & 68 & 84 & 81 & 80 & 75 & 86 & 87 & 84 & 87 & 68 \\
\hline 07 & 92 & 85 & 89 & 67 & 91 & 79 & 98 & 88 & 86 & 85 & 89 & 84 & 79 \\
\hline 08 & 96 & 79 & 84 & 65 & 80 & 74 & 88 & 97 & 82 & 81 & 84 & 80 & 85 \\
\hline 73 & 85 & 91 & 92 & 71 & 92 & 86 & 88 & 83 & 92 & 92 & 92 & 92 & 74 \\
\hline 74 & 84 & 91 & 91 & 71 & 90 & 86 & 86 & 82 & 92 & 92 & 91 & 92 & 73 \\
\hline 75 & 88 & 89 & 92 & 68 & 91 & 83 & 90 & 85 & 91 & 90 & 92 & 89 & 75 \\
\hline 76 & 83 & 91 & 91 & 72 & 90 & 87 & 85 & 81 & 92 & 92 & 90 & 93 & 73 \\
\hline DS & 85 & 71 & 75 & 58 & 72 & 67 & 79 & 84 & 73 & 72 & 74 & 72 & - \\
\hline
\end{tabular}


Table B-164. Pennsylvania Site 7 Left, Medium Wavelengths.

\begin{tabular}{|c|c|c|c|c|c|c|c|c|c|c|c|c|c|}
\hline $\begin{array}{c}\text { Correlation } \\
\text { Reference }\end{array}$ & 01 & 02 & 03 & 04 & 05 & 06 & 07 & 08 & 73 & 74 & 75 & 76 & DS \\
\hline 01 & 93 & 83 & 76 & 89 & 85 & 70 & 85 & 89 & 83 & 85 & 82 & 82 & 71 \\
\hline 02 & 88 & 81 & 78 & 86 & 82 & 68 & 82 & 83 & 80 & 81 & 81 & 81 & 71 \\
\hline 03 & 76 & 69 & 86 & 80 & 73 & 59 & 75 & 71 & 72 & 72 & 71 & 75 & 76 \\
\hline 04 & 89 & 80 & 82 & 92 & 83 & 70 & 85 & 85 & 83 & 84 & 81 & 84 & 72 \\
\hline 05 & 87 & 87 & 78 & 86 & 95 & 69 & 83 & 77 & 83 & 81 & 81 & 81 & 68 \\
\hline 06 & 73 & 64 & 65 & 72 & 66 & 56 & 69 & 72 & 67 & 69 & 67 & 69 & 63 \\
\hline 07 & 86 & 78 & 79 & 85 & 83 & 66 & 82 & 82 & 78 & 79 & 80 & 80 & 71 \\
\hline 08 & 88 & 75 & 73 & 83 & 76 & 66 & 81 & 90 & 76 & 79 & 81 & 80 & 72 \\
\hline 73 & 85 & 75 & 80 & 84 & 77 & 66 & 81 & 82 & 80 & 79 & 79 & 80 & 75 \\
\hline 74 & 85 & 75 & 78 & 83 & 76 & 65 & 80 & 83 & 76 & 82 & 78 & 79 & 76 \\
\hline 75 & 85 & 74 & 75 & 81 & 75 & 63 & 79 & 82 & 76 & 77 & 79 & 78 & 76 \\
\hline 76 & 82 & 73 & 79 & 83 & 74 & 64 & 78 & 81 & 75 & 77 & 77 & 82 & 76 \\
\hline DS & 72 & 63 & 68 & 71 & 66 & 56 & 69 & 70 & 66 & 67 & 67 & 67 & - \\
\hline
\end{tabular}

Table B-165. Pennsylvania Site 7 Left, Short Wavelengths.

\begin{tabular}{|c|c|c|c|c|c|c|c|c|c|c|c|c|c|}
\hline $\begin{array}{c}\text { Correlation } \\
\text { Reference }\end{array}$ & 01 & 02 & 03 & 04 & 05 & 06 & 07 & 08 & 73 & 74 & 75 & 76 & DS \\
\hline 01 & 67 & 52 & 32 & 33 & 54 & 42 & 33 & 49 & 53 & 61 & 55 & 52 & 30 \\
\hline 02 & 43 & 53 & 38 & 26 & 49 & 34 & 26 & 35 & 48 & 48 & 48 & 49 & 46 \\
\hline 03 & 18 & 30 & 54 & 19 & 32 & 21 & 17 & 14 & 36 & 35 & 31 & 38 & 36 \\
\hline 04 & 45 & 47 & 49 & 50 & 58 & 39 & 32 & 32 & 51 & 56 & 48 & 55 & 37 \\
\hline 05 & 56 & 56 & 48 & 38 & 82 & 40 & 40 & 39 & 55 & 54 & 56 & 60 & 54 \\
\hline 06 & 33 & 33 & 27 & 21 & 34 & 31 & 20 & 28 & 37 & 37 & 35 & 39 & 41 \\
\hline 07 & 37 & 44 & 34 & 27 & 40 & 32 & 23 & 28 & 44 & 43 & 45 & 45 & 35 \\
\hline 08 & 47 & 39 & 25 & 19 & 34 & 32 & 22 & 52 & 38 & 43 & 47 & 45 & 35 \\
\hline 73 & 38 & 42 & 39 & 26 & 45 & 34 & 25 & 29 & 54 & 45 & 47 & 47 & 51 \\
\hline 74 & 38 & 39 & 35 & 25 & 43 & 31 & 24 & 31 & 42 & 56 & 44 & 45 & 47 \\
\hline 75 & 40 & 39 & 31 & 22 & 40 & 29 & 23 & 34 & 44 & 44 & 53 & 43 & 48 \\
\hline 76 & 33 & 38 & 35 & 25 & 40 & 30 & 22 & 27 & 41 & 42 & 40 & 54 & 46 \\
\hline DS & 30 & 31 & 29 & 20 & 35 & 26 & 20 & 23 & 36 & 33 & 35 & 36 & - \\
\hline
\end{tabular}


Table B-166. Pennsylvania Site 7 Right, IRI Filter.

\begin{tabular}{|c|c|c|c|c|c|c|c|c|c|c|c|c|c|}
\hline $\begin{array}{c}\text { Correlation } \\
\text { Reference }\end{array}$ & 01 & 02 & 03 & 04 & 05 & 06 & 07 & 08 & 73 & 74 & 75 & 76 & DS \\
\hline 01 & 91 & 85 & 76 & 85 & 87 & 85 & 88 & 87 & 82 & 84 & 83 & 83 & 78 \\
\hline 02 & 88 & 85 & 79 & 83 & 87 & 82 & 87 & 86 & 83 & 82 & 83 & 83 & 79 \\
\hline 03 & 76 & 73 & 86 & 80 & 84 & 74 & 81 & 76 & 76 & 74 & 74 & 78 & 81 \\
\hline 04 & 85 & 82 & 81 & 91 & 91 & 83 & 91 & 82 & 82 & 82 & 80 & 85 & 78 \\
\hline 05 & 87 & 81 & 84 & 89 & $\mathbf{9 6}$ & 82 & 93 & 88 & 82 & 81 & 83 & 86 & 86 \\
\hline 06 & 87 & 83 & 81 & 85 & 89 & 85 & 89 & 86 & 84 & 83 & 83 & 85 & 80 \\
\hline 07 & 89 & 84 & 84 & 90 & 93 & 85 & $\mathbf{9 4}$ & 87 & 84 & 83 & 84 & 88 & 82 \\
\hline 08 & 88 & 80 & 74 & 81 & 86 & 80 & 85 & $\mathbf{9 5}$ & 79 & 79 & 82 & 81 & 83 \\
\hline 73 & 85 & 80 & 80 & 82 & 87 & 81 & 86 & 86 & 83 & 79 & 81 & 81 & 82 \\
\hline 74 & 86 & 82 & 80 & 84 & 88 & 82 & 87 & 86 & 82 & 84 & 82 & 83 & 81 \\
\hline 75 & 84 & 79 & 77 & 80 & 86 & 79 & 84 & 86 & 80 & 78 & 81 & 80 & 82 \\
\hline 76 & 85 & 80 & 82 & 85 & 91 & 81 & 88 & 87 & 81 & 80 & 81 & 86 & 82 \\
\hline DS & 78 & 72 & 74 & 76 & 83 & 73 & 80 & 81 & 74 & 72 & 74 & 75 & - \\
\hline
\end{tabular}

Table B-167. Pennsylvania Site 7 Right, Long Wavelengths.

\begin{tabular}{|c|c|c|c|c|c|c|c|c|c|c|c|c|c|}
\hline $\begin{array}{c}\text { Correlation } \\
\text { Reference }\end{array}$ & 01 & 02 & 03 & 04 & 05 & 06 & 07 & 08 & 73 & 74 & 75 & 76 & DS \\
\hline 01 & 96 & 87 & 90 & 70 & 89 & 84 & 89 & 93 & 87 & 87 & 90 & 88 & 87 \\
\hline 02 & 88 & 93 & 92 & 71 & 84 & 92 & 87 & 82 & 93 & 92 & 92 & 92 & 78 \\
\hline 03 & 91 & 91 & 95 & 71 & 88 & 90 & 91 & 86 & 93 & 92 & 93 & 93 & 82 \\
\hline 04 & 70 & 71 & 71 & 61 & 69 & 72 & 71 & 67 & 72 & 72 & 70 & 73 & 64 \\
\hline 05 & 90 & 83 & 87 & 69 & 99 & 82 & 96 & 89 & 85 & 86 & 86 & 87 & 86 \\
\hline 06 & 85 & 93 & 91 & 73 & 83 & 94 & 86 & 81 & 93 & 92 & 91 & 93 & 77 \\
\hline 07 & 89 & 86 & 90 & 70 & 96 & 85 & 98 & 85 & 88 & 89 & 89 & 90 & 82 \\
\hline 08 & 93 & 82 & 85 & 67 & 89 & 80 & 85 & 98 & 83 & 83 & 85 & 83 & 92 \\
\hline 73 & 89 & 93 & 94 & 72 & 86 & 92 & 89 & 84 & 94 & 93 & 93 & 94 & 80 \\
\hline 74 & 88 & 92 & 93 & 72 & 87 & 92 & 90 & 84 & 93 & 92 & 92 & 93 & 80 \\
\hline 75 & 91 & 91 & 93 & 71 & 88 & 90 & 90 & 86 & 92 & 92 & 92 & 92 & 82 \\
\hline 76 & 89 & 92 & 94 & 73 & 88 & 92 & 91 & 84 & 94 & 93 & 93 & 95 & 81 \\
\hline DS & 87 & 77 & 81 & 64 & 85 & 76 & 82 & 92 & 79 & 79 & 80 & 80 & - \\
\hline
\end{tabular}


Table B-168. Pennsylvania Site 7 Right, Medium Wavelengths.

\begin{tabular}{|c|c|c|c|c|c|c|c|c|c|c|c|c|c|}
\hline $\begin{array}{c}\text { Correlation } \\
\text { Reference }\end{array}$ & 01 & 02 & 03 & 04 & 05 & 06 & 07 & 08 & 73 & 74 & 75 & 76 & DS \\
\hline 01 & 90 & 83 & 73 & 84 & 86 & 83 & 87 & 87 & 79 & 83 & 80 & 81 & 76 \\
\hline 02 & 86 & 82 & 76 & 83 & 86 & 78 & 85 & 86 & 79 & 78 & 80 & 80 & 80 \\
\hline 03 & 72 & 70 & 83 & 78 & 82 & 70 & 78 & 73 & 72 & 71 & 71 & 74 & 80 \\
\hline 04 & 85 & 81 & 80 & 93 & 92 & 82 & 91 & 84 & 81 & 81 & 81 & 85 & 79 \\
\hline 05 & 85 & 79 & 81 & 89 & 95 & 79 & 92 & 88 & 79 & 78 & 80 & 84 & 86 \\
\hline 06 & 85 & 79 & 77 & 85 & 88 & 82 & 87 & 87 & 81 & 80 & 80 & 82 & 80 \\
\hline 07 & 87 & 82 & 81 & 90 & 92 & 82 & 92 & 88 & 82 & 81 & 83 & 86 & 82 \\
\hline 08 & 87 & 79 & 72 & 81 & 87 & 79 & 86 & 94 & 78 & 77 & 81 & 81 & 81 \\
\hline 73 & 82 & 76 & 77 & 81 & 86 & 78 & 84 & 84 & 80 & 76 & 79 & 78 & 82 \\
\hline 74 & 84 & 78 & 77 & 83 & 87 & 79 & 86 & 86 & 79 & 81 & 79 & 80 & 81 \\
\hline 75 & 82 & 75 & 74 & 79 & 84 & 75 & 82 & 85 & 77 & 74 & 79 & 77 & 83 \\
\hline 76 & 82 & 77 & 79 & 84 & 89 & 78 & 86 & 85 & 78 & 77 & 79 & 84 & 82 \\
\hline DS & 76 & 70 & 71 & 76 & 82 & 71 & 80 & 79 & 72 & 70 & 72 & 73 & - \\
\hline
\end{tabular}

Table B-169. Pennsylvania Site 7 Right, Short Wavelengths.

\begin{tabular}{|c|c|c|c|c|c|c|c|c|c|c|c|c|c|}
\hline $\begin{array}{c}\text { Correlation } \\
\text { Reference }\end{array}$ & 01 & 02 & 03 & 04 & 05 & 06 & 07 & 08 & 73 & 74 & 75 & 76 & DS \\
\hline 01 & 69 & 50 & 32 & 35 & 45 & 48 & 45 & 49 & 51 & 58 & 54 & 53 & 40 \\
\hline 02 & 41 & 54 & 32 & 27 & 43 & 39 & 44 & 47 & 45 & 45 & 47 & 48 & 51 \\
\hline 03 & 19 & 28 & 51 & 22 & 33 & 26 & 29 & 19 & 35 & 35 & 32 & 37 & 39 \\
\hline 04 & 43 & 45 & 46 & 58 & 65 & 47 & 57 & 39 & 51 & 52 & 50 & 56 & 51 \\
\hline 05 & 43 & 44 & 37 & 39 & 69 & 43 & 58 & 47 & 47 & 45 & 47 & 55 & 63 \\
\hline 06 & 40 & 41 & 32 & 28 & 45 & 49 & 45 & 50 & 48 & 47 & 44 & 49 & 56 \\
\hline 07 & 45 & 45 & 35 & 37 & 58 & 44 & 55 & 50 & 47 & 47 & 48 & 56 & 56 \\
\hline 08 & 47 & 41 & 24 & 21 & 38 & 41 & 42 & 75 & 41 & 44 & 46 & 50 & 48 \\
\hline 73 & 35 & 39 & 34 & 26 & 41 & 40 & 41 & 41 & 52 & 42 & 47 & 44 & 51 \\
\hline 74 & 36 & 38 & 33 & 26 & 42 & 39 & 42 & 45 & 42 & 56 & 43 & 46 & 48 \\
\hline 75 & 38 & 39 & 30 & 24 & 39 & 35 & 40 & 46 & 45 & 42 & 52 & 43 & 50 \\
\hline 76 & 34 & 39 & 33 & 28 & 45 & 38 & 43 & 42 & 41 & 44 & 42 & 60 & 46 \\
\hline DS & 35 & 37 & 33 & 25 & 45 & 39 & 45 & 43 & 43 & 39 & 42 & 42 & - \\
\hline
\end{tabular}




\begin{tabular}{|c|c|c|c|c|c|c|c|c|c|c|c|c|c|}
\hline- & $8 \mathrm{~L}$ & $6 L$ & $6 L$ & $8 \mathrm{~L}$ & 26 & 28 & $8 L$ & $6 L$ & $0 t$ & $6 L$ & $L L$ & $\varepsilon 6$ & $\mathrm{SA}$ \\
\hline 08 & 28 & $\varepsilon 8$ & 78 & 78 & $6 L$ & I8 & 28 & 58 & $z t$ & $\varepsilon 8$ & 28 & I8 & $9 \mathrm{~L}$ \\
\hline I8 & $\varepsilon 8$ & t8 & 58 & $\$ 8$ & 08 & 28 & 28 & 98 & It & $\varsigma 8$ & $\varepsilon 8$ & $\varepsilon 8$ & $S L$ \\
\hline 08 & 78 & 58 & 98 & $\angle 8$ & $6 L$ & $\varepsilon 8$ & $\varepsilon 8$ & 88 & $\varepsilon t$ & 98 & 78 & 28 & $t L$ \\
\hline $6 L$ & t8 & 98 & $\angle 8$ & $\angle 8$ & $6 \mathrm{~L}$ & 28 & $\varepsilon 8$ & 06 & $\varepsilon t$ & $\angle 8$ & $\$ 8$ & 28 & $\varepsilon L$ \\
\hline I6 & $L L$ & $6 \mathrm{~L}$ & $8 L$ & $L L$ & $\subseteq 6$ & 28 & $\nabla L$ & $6 L$ & $0 t$ & $8 \mathrm{~L}$ & $L L$ & $\$ 6$ & 80 \\
\hline$\varepsilon 8$ & 08 & I8 & I8 & I8 & 28 & 06 & $9 L$ & $\angle 8$ & $\angle \mathcal{E}$ & I8 & $6 L$ & 98 & $\angle 0$ \\
\hline $6 L$ & 28 & $\varepsilon 8$ & 78 & 78 & $S L$ & $8 \mathrm{~L}$ & 88 & 28 & $\varepsilon t$ & $\varepsilon 8$ & I8 & $8 L$ & 90 \\
\hline 08 & $\$ 8$ & $\angle 8$ & 88 & 68 & $6 \mathrm{~L}$ & $\angle 8$ & I8 & 66 & It & 68 & 98 & $\varepsilon 8$ & $\varsigma 0$ \\
\hline $0 t$ & $z t$ & It & $z t$ & $2 t$ & $0 t$ & $\angle \mathcal{E}$ & $\varepsilon t$ & It & $\tau \mathcal{E}$ & It & It & It & t0 \\
\hline I8 & $\varepsilon 8$ & $\$ 8$ & 58 & 98 & 08 & 28 & 28 & 88 & It & 88 & 78 & $\varepsilon 8$ & $\varepsilon 0$ \\
\hline $6 L$ & 28 & $\varepsilon 8$ & 78 & $\$ 8$ & $8 \mathrm{~L}$ & 08 & I8 & 98 & It & $\$ 8$ & 28 & I8 & 20 \\
\hline 26 & 08 & 28 & I8 & 08 & 76 & 98 & LL & $\varepsilon 8$ & It & 28 & 08 & 96 & I0 \\
\hline SQ & $9 L$ & $S L$ & $t L$ & $\varepsilon L$ & $\begin{array}{l}80 \\
\text { วัน } \Lambda \partial\end{array}$ & $\left\{\begin{array}{l}\angle 0 \\
\text { pəI }\end{array}\right.$ & 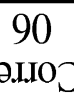 & $\varsigma_{0}$ & t0 & $\varepsilon 0$ & 20 & I0 & 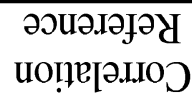 \\
\hline
\end{tabular}

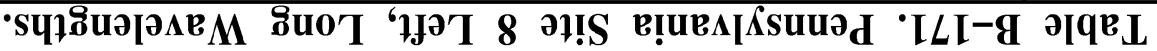

\begin{tabular}{|c|c|c|c|c|c|c|c|c|c|c|c|c|c|}
\hline- & 99 & 89 & 69 & 89 & $Z L$ & $\angle S$ & $\$ 9$ & IL & $Z L$ & 89 & $\varepsilon 9$ & $L L$ & SA \\
\hline$\nabla L$ & $\varepsilon L$ & $\varepsilon L$ & $\nabla L$ & $\nabla L$ & $8 \mathrm{~L}$ & I9 & 69 & $t L$ & $9 L$ & 69 & 69 & 08 & $9 L$ \\
\hline$L L$ & 69 & $9 L$ & $\nabla L$ & $S L$ & $8 \mathrm{~L}$ & I9 & $\$ 9$ & $L L$ & $\varepsilon L$ & $0 L$ & 89 & $\$ 8$ & $S L$ \\
\hline$L L$ & IL & $S L$ & $L L$ & $S L$ & $8 \mathrm{~L}$ & $\varepsilon 9$ & $\angle 9$ & $L L$ & $\nabla L$ & $\mathrm{IL}$ & 69 & $\$ 8$ & $t L$ \\
\hline$L L$ & $0 L$ & $S L$ & $S L$ & $L L$ & LL & $\varepsilon 9$ & 99 & $6 \mathrm{~L}$ & $S L$ & $\nabla L$ & $0 L$ & 78 & $\varepsilon L$ \\
\hline$\nabla L$ & $9 L$ & $6 L$ & $6 L$ & $6 L$ & 88 & 89 & 69 & I8 & $6 L$ & $\mathrm{IL}$ & $\nabla L$ & 98 & 80 \\
\hline 09 & 79 & $\angle 9$ & 69 & $\angle 9$ & $0 L$ & 09 & 09 & 89 & 99 & t9 & $\$ 9$ & 89 & $\angle 0$ \\
\hline$\tau L$ & $\angle 9$ & $\angle 9$ & $\angle 9$ & $\angle 9$ & 89 & $9 \varsigma$ & $t L$ & 99 & $\varepsilon L$ & $\angle 9$ & 29 & $\mathrm{IL}$ & 90 \\
\hline$\nabla L$ & $\mathrm{IL}$ & $6 L$ & $L L$ & $6 L$ & I8 & 89 & 99 & 96 & $8 L$ & I8 & $S L$ & 98 & $\subseteq 0$ \\
\hline$S L$ & $\varepsilon L$ & $\mathrm{I} L$ & $\varepsilon L$ & $S L$ & $8 \mathrm{~L}$ & t9 & $Z L$ & $L L$ & I8 & $\varepsilon L$ & $0 L$ & $8 L$ & t0 \\
\hline$S L$ & $\$ 9$ & $0 L$ & 69 & $\varepsilon L$ & 69 & 85 & 99 & 08 & $\nabla L$ & $\varepsilon 8$ & 89 & $9 L$ & $\varepsilon 0$ \\
\hline $0 L$ & $\mathrm{IL}$ & $t L$ & $\nabla L$ & $S L$ & $L L$ & $\varepsilon 9$ & 99 & 08 & $\nabla L$ & $\nabla L$ & $\varepsilon L$ & $6 L$ & 20 \\
\hline $8 \mathrm{~L}$ & $\nabla L$ & $8 L$ & $8 L$ & 08 & 78 & 99 & $\angle 9$ & 78 & $L L$ & $Z L$ & $\varepsilon L$ & $\varepsilon 6$ & $\mathrm{I0}$ \\
\hline $\mathrm{SC}$ & $9 L$ & $S L$ & $t L$ & $\varepsilon L$ & 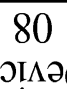 & $\begin{array}{l}\angle 0 \\
\text { pəIx }\end{array}$ & $\begin{array}{c}90 \\
. \mathrm{L}^{\prime}\end{array}$ & $\varsigma_{0}$ & t0 & $\varepsilon 0$ & 20 & $\mathrm{I0}$ & 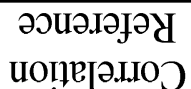 \\
\hline
\end{tabular}


Table B-172. Pennsylvania Site 8 Left, Medium Wavelengths.

\begin{tabular}{|c|c|c|c|c|c|c|c|c|c|c|c|c|c|}
\hline $\begin{array}{c}\text { Correlation } \\
\text { Reference }\end{array}$ & 01 & 02 & 03 & 04 & 05 & 06 & 07 & 08 & 73 & 74 & 75 & 76 & DS \\
\hline 01 & 93 & 70 & 69 & 79 & 83 & 65 & 57 & 82 & 78 & 76 & 77 & 73 & 76 \\
\hline 02 & 80 & 72 & 71 & 75 & 79 & 63 & 53 & 74 & 73 & 71 & 72 & 68 & 71 \\
\hline 03 & 72 & 64 & 81 & 72 & 77 & 61 & 48 & 64 & 68 & 64 & 65 & 61 & 74 \\
\hline 04 & 80 & 70 & 73 & 85 & 79 & 73 & 57 & 78 & 76 & 74 & 72 & 74 & 77 \\
\hline 05 & 86 & 72 & 79 & 79 & 96 & 63 & 58 & 78 & 76 & 72 & 76 & 69 & 73 \\
\hline 06 & 69 & 58 & 63 & 73 & 64 & 71 & 47 & 65 & 64 & 64 & 64 & 64 & 72 \\
\hline 07 & 59 & 59 & 57 & 60 & 58 & 54 & 51 & 63 & 61 & 62 & 61 & 58 & 52 \\
\hline 08 & 84 & 71 & 67 & 78 & 79 & 67 & 58 & 86 & 76 & 77 & 77 & 74 & 71 \\
\hline 73 & 83 & 67 & 70 & 75 & 78 & 63 & 53 & 74 & 76 & 71 & 73 & 68 & 77 \\
\hline 74 & 84 & 66 & 66 & 75 & 75 & 64 & 53 & 75 & 71 & 75 & 72 & 69 & 78 \\
\hline 75 & 83 & 65 & 65 & 72 & 75 & 62 & 52 & 74 & 71 & 70 & 74 & 66 & 77 \\
\hline 76 & 80 & 65 & 66 & 76 & 72 & 66 & 52 & 75 & 70 & 71 & 70 & 72 & 75 \\
\hline DS & 74 & 60 & 64 & 72 & 69 & 61 & 48 & 68 & 64 & 66 & 65 & 64 & - \\
\hline
\end{tabular}

Table B-173. Pennsylvania Site 8 Left, Short Wavelengths.

\begin{tabular}{|c|c|c|c|c|c|c|c|c|c|c|c|c|c|}
\hline $\begin{array}{c}\text { Correlation } \\
\text { Reference }\end{array}$ & 01 & 02 & 03 & 04 & 05 & 06 & 07 & 08 & 73 & 74 & 75 & 76 & DS \\
\hline 01 & 75 & 45 & 30 & 39 & 51 & 39 & 13 & 55 & 54 & 54 & 55 & 53 & 55 \\
\hline 02 & 45 & 52 & 36 & 29 & 49 & 31 & 9 & 36 & 45 & 43 & 45 & 42 & 47 \\
\hline 03 & 21 & 29 & 58 & 21 & 42 & 22 & 5 & 17 & 34 & 28 & 29 & 27 & 33 \\
\hline 04 & 51 & 44 & 41 & 56 & 55 & 46 & 12 & 44 & 54 & 53 & 48 & 53 & 61 \\
\hline 05 & 49 & 44 & 48 & 34 & 84 & 32 & 12 & 36 & 47 & 42 & 46 & 41 & 47 \\
\hline 06 & 34 & 29 & 27 & 26 & 33 & 43 & 8 & 28 & 36 & 36 & 35 & 35 & 43 \\
\hline 07 & 18 & 30 & 20 & 19 & 12 & 23 & 13 & 16 & 31 & 32 & 32 & 28 & 14 \\
\hline 08 & 60 & 42 & 28 & 32 & 42 & 36 & 11 & 54 & 49 & 51 & 52 & 48 & 40 \\
\hline 73 & 44 & 39 & 36 & 29 & 48 & 33 & 9 & 36 & 56 & 44 & 48 & 41 & 47 \\
\hline 74 & 47 & 37 & 28 & 27 & 41 & 33 & 9 & 38 & 43 & 57 & 43 & 43 & 51 \\
\hline 75 & 45 & 37 & 29 & 26 & 40 & 30 & 9 & 37 & 45 & 42 & 54 & 40 & 45 \\
\hline 76 & 42 & 35 & 28 & 27 & 37 & 32 & 8 & 36 & 40 & 42 & 41 & 51 & 52 \\
\hline DS & 37 & 32 & 29 & 29 & 39 & 30 & 8 & 31 & 36 & 40 & 37 & 39 & - \\
\hline
\end{tabular}


Table B-174. Pennsylvania Site 8 Right, IRI Filter.

\begin{tabular}{|c|c|c|c|c|c|c|c|c|c|c|c|c|c|}
\hline $\begin{array}{c}\text { Correlation } \\
\text { Reference }\end{array}$ & 01 & 02 & 03 & 04 & 05 & 06 & 07 & 08 & 73 & 74 & 75 & 76 & DS \\
\hline 01 & 88 & 69 & 64 & 70 & 75 & 68 & 80 & 81 & 74 & 72 & 73 & 70 & 72 \\
\hline 02 & 75 & 70 & 66 & 67 & 73 & 67 & 73 & 71 & 72 & 68 & 69 & 68 & 64 \\
\hline 03 & 66 & 61 & 71 & 63 & 75 & 60 & 72 & 67 & 66 & 59 & 61 & 59 & 68 \\
\hline 04 & 71 & 64 & 65 & 78 & 81 & 77 & 79 & 72 & 69 & 65 & 66 & 69 & 69 \\
\hline 05 & 76 & 65 & 74 & 79 & 93 & 73 & 89 & 79 & 73 & 66 & 68 & 68 & 77 \\
\hline 06 & 75 & 64 & 65 & 79 & 81 & 79 & 81 & 75 & 72 & 69 & 69 & 70 & 72 \\
\hline 07 & 80 & 66 & 69 & 77 & 89 & 73 & $\mathbf{9 4}$ & 87 & 74 & 70 & 71 & 71 & 84 \\
\hline 08 & 81 & 65 & 64 & 70 & 78 & 69 & 87 & 88 & 74 & 71 & 72 & 70 & 79 \\
\hline 73 & 77 & 65 & 68 & 68 & 77 & 66 & 81 & 79 & 74 & 68 & 70 & 67 & 73 \\
\hline 74 & 77 & 64 & 62 & 66 & 72 & 66 & 78 & 77 & 71 & 70 & 70 & 68 & 70 \\
\hline 75 & 78 & 64 & 64 & 66 & 73 & 66 & 78 & 78 & 72 & 68 & 71 & 66 & 71 \\
\hline 76 & 76 & 65 & 63 & 70 & 75 & 70 & 79 & 77 & 72 & 70 & 70 & 71 & 71 \\
\hline DS & 70 & 57 & 61 & 66 & 75 & 64 & 82 & 76 & 64 & 62 & 62 & 63 & - \\
\hline
\end{tabular}

Table B-175. Pennsylvania Site 8 Right, Long Wavelengths.

\begin{tabular}{|c|c|c|c|c|c|c|c|c|c|c|c|c|c|}
\hline $\begin{array}{c}\text { Correlation } \\
\text { Reference }\end{array}$ & 01 & 02 & 03 & 04 & 05 & 06 & 07 & 08 & 73 & 74 & 75 & 76 & DS \\
\hline 01 & 92 & 81 & 83 & 47 & 79 & 81 & 81 & 86 & 83 & 85 & 83 & 82 & 80 \\
\hline 02 & 82 & 86 & 84 & 51 & 76 & 82 & 79 & 75 & 86 & 86 & 86 & 85 & 73 \\
\hline 03 & 83 & 83 & 84 & 49 & 79 & 83 & 82 & 77 & 85 & 85 & 84 & 83 & 75 \\
\hline 04 & 47 & 51 & 49 & 38 & 47 & 51 & 48 & 45 & 50 & 50 & 50 & 51 & 44 \\
\hline 05 & 80 & 75 & 79 & 47 & 98 & 85 & 95 & 83 & 79 & 79 & 76 & 79 & 84 \\
\hline 06 & 82 & 81 & 83 & 51 & 86 & 90 & 88 & 79 & 85 & 85 & 83 & 85 & 80 \\
\hline 07 & 82 & 78 & 81 & 48 & 95 & 87 & 97 & 81 & 82 & 83 & 79 & 81 & 82 \\
\hline 08 & 86 & 74 & 76 & 45 & 83 & 78 & 81 & 93 & 76 & 77 & 76 & 76 & 91 \\
\hline 73 & 84 & 85 & 85 & 50 & 80 & 85 & 83 & 78 & 86 & 87 & 86 & 85 & 75 \\
\hline 74 & 86 & 86 & 85 & 50 & 80 & 86 & 84 & 79 & 87 & 88 & 87 & 86 & 76 \\
\hline 75 & 84 & 86 & 84 & 50 & 76 & 84 & 79 & 77 & 86 & 87 & 87 & 85 & 74 \\
\hline 76 & 82 & 85 & 84 & 51 & 80 & 85 & 82 & 77 & 85 & 86 & 85 & 85 & 75 \\
\hline DS & 80 & 72 & 74 & 45 & 84 & 78 & 82 & 90 & 74 & 76 & 73 & 74 & - \\
\hline
\end{tabular}


Table B-176. Pennsylvania Site 8 Right, Medium Wavelengths.

\begin{tabular}{|c|c|c|c|c|c|c|c|c|c|c|c|c|c|}
\hline $\begin{array}{c}\text { Correlation } \\
\text { Reference }\end{array}$ & 01 & 02 & 03 & 04 & 05 & 06 & 07 & 08 & 73 & 74 & 75 & 76 & DS \\
\hline 01 & 89 & 65 & 57 & 72 & 73 & 66 & 82 & 79 & 71 & 68 & 71 & 67 & 73 \\
\hline 02 & 74 & 66 & 61 & 67 & 71 & 61 & 75 & 72 & 66 & 61 & 64 & 60 & 65 \\
\hline 03 & 59 & 54 & 69 & 61 & 70 & 53 & 66 & 60 & 58 & 50 & 53 & 50 & 66 \\
\hline 04 & 73 & 66 & 64 & 84 & 83 & 78 & 83 & 72 & 70 & 66 & 68 & 69 & 70 \\
\hline 05 & 74 & 62 & 69 & 79 & 90 & 69 & 86 & 76 & 68 & 61 & 65 & 63 & 75 \\
\hline 06 & 75 & 59 & 60 & 78 & 79 & 77 & 83 & 76 & 67 & 64 & 66 & 64 & 74 \\
\hline 07 & 82 & 64 & 62 & 78 & 86 & 70 & 93 & 87 & 70 & 66 & 69 & 68 & 83 \\
\hline 08 & 79 & 61 & 55 & 69 & 73 & 65 & 84 & 87 & 68 & 66 & 68 & 65 & 78 \\
\hline 73 & 73 & 59 & 60 & 67 & 73 & 61 & 78 & 76 & 70 & 61 & 65 & 61 & 75 \\
\hline 74 & 75 & 58 & 54 & 65 & 68 & 61 & 77 & 76 & 64 & 66 & 64 & 61 & 72 \\
\hline 75 & 75 & 57 & 55 & 64 & 68 & 60 & 77 & 77 & 65 & 61 & 68 & 60 & 73 \\
\hline 76 & 75 & 60 & 56 & 69 & 73 & 65 & 81 & 76 & 67 & 64 & 65 & 68 & 73 \\
\hline DS & 69 & 54 & 55 & 67 & 72 & 62 & 80 & 74 & 60 & 59 & 60 & 60 & - \\
\hline
\end{tabular}

Table B-177. Pennsylvania Site 8 Right, Short Wavelengths.

\begin{tabular}{|c|c|c|c|c|c|c|c|c|c|c|c|c|c|}
\hline $\begin{array}{c}\text { Correlation } \\
\text { Reference }\end{array}$ & 01 & 02 & 03 & 04 & 05 & 06 & 07 & 08 & 73 & 74 & 75 & 76 & DS \\
\hline 01 & 74 & 44 & 26 & 42 & 44 & 47 & 66 & 56 & 52 & 49 & 55 & 49 & 62 \\
\hline 02 & 45 & 50 & 32 & 34 & 42 & 38 & 52 & 48 & 41 & 38 & 41 & 40 & 48 \\
\hline 03 & 20 & 28 & 53 & 26 & 39 & 26 & 32 & 24 & 31 & 23 & 26 & 23 & 31 \\
\hline 04 & 53 & 51 & 44 & 64 & 66 & 59 & 71 & 48 & 55 & 49 & 54 & 53 & 55 \\
\hline 05 & 46 & 43 & 44 & 47 & 73 & 45 & 63 & 43 & 47 & 38 & 44 & 43 & 49 \\
\hline 06 & 47 & 38 & 30 & 41 & 47 & 57 & 59 & 55 & 45 & 42 & 45 & 44 & 57 \\
\hline 07 & 62 & 44 & 32 & 47 & 63 & 48 & 84 & 68 & 50 & 46 & 52 & 50 & 62 \\
\hline 08 & 54 & 38 & 22 & 33 & 36 & 40 & 58 & 68 & 43 & 43 & 47 & 45 & 49 \\
\hline 73 & 45 & 38 & 32 & 36 & 44 & 41 & 55 & 51 & 56 & 40 & 49 & 43 & 52 \\
\hline 74 & 48 & 36 & 24 & 33 & 38 & 40 & 55 & 55 & 42 & 52 & 44 & 42 & 53 \\
\hline 75 & 46 & 34 & 25 & 31 & 37 & 38 & 52 & 53 & 45 & 39 & 55 & 40 & 48 \\
\hline 76 & 47 & 37 & 24 & 34 & 41 & 40 & 57 & 56 & 44 & 41 & 45 & 55 & 56 \\
\hline DS & 45 & 34 & 26 & 36 & 45 & 40 & 62 & 52 & 39 & 40 & 42 & 43 & - \\
\hline
\end{tabular}


Table B-178. South Dakota Site 1 Left, IRI Filter.

\begin{tabular}{|c|c|c|c|c|c|c|c|c|c|c|c|}
\hline $\begin{array}{c}\text { Correlation } \\
\text { Reference }\end{array}$ & \multicolumn{10}{|c|}{ Correlated Device } \\
\hline 01 & 81 & 74 & 71 & 71 & 67 & 71 & 69 & 33 & 63 & 74 & 81 \\
\hline 02 & 68 & 83 & 71 & 68 & 75 & 67 & 77 & 25 & 61 & 73 & 83 \\
\hline 03 & 67 & 75 & 70 & 67 & 70 & 63 & 72 & 25 & 60 & 70 & 76 \\
\hline 04 & 67 & 68 & 68 & 67 & 68 & 64 & 70 & 26 & 56 & 69 & 70 \\
\hline 05 & 62 & 71 & 64 & 64 & $\mathbf{9 4}$ & 56 & 93 & 23 & 53 & 65 & 70 \\
\hline 06 & 70 & 67 & 67 & 64 & 60 & 73 & 64 & 31 & 61 & 66 & 68 \\
\hline 08 & 64 & 72 & 66 & 66 & 93 & 60 & $\mathbf{9 5}$ & 25 & 54 & 67 & 71 \\
\hline 10 & 37 & 28 & 29 & 28 & 25 & 34 & 27 & 90 & 26 & 28 & 33 \\
\hline 11 & 68 & 67 & 65 & 61 & 57 & 65 & 59 & 25 & 70 & 66 & 68 \\
\hline 12 & 70 & 73 & 70 & 68 & 69 & 66 & 71 & 25 & 61 & 71 & 77 \\
\hline DS & 75 & 83 & 75 & 70 & 74 & 68 & 75 & 29 & 62 & 76 & - \\
\hline
\end{tabular}

Table B-179. South Dakota Site 1 Left, Long Wavelengths.

\begin{tabular}{|c|c|c|c|c|c|c|c|c|c|c|c|}
\hline $\begin{array}{c}\text { Correlation } \\
\text { Reference }\end{array}$ & \multicolumn{10}{|c|}{ Correlated Device } \\
\hline 01 & 98 & 77 & 75 & 77 & 80 & 79 & 82 & 86 & 71 & 77 & 87 \\
\hline 02 & 76 & 97 & 95 & 94 & 87 & 94 & 86 & 67 & 64 & 96 & 81 \\
\hline 03 & 74 & 95 & 95 & 94 & 89 & 92 & 87 & 64 & 66 & 95 & 79 \\
\hline 04 & 77 & 94 & 94 & 94 & 90 & 93 & 88 & 66 & 63 & 95 & 82 \\
\hline 05 & 79 & 88 & 89 & 90 & 98 & 90 & 97 & 66 & 69 & 89 & 84 \\
\hline 06 & 79 & 94 & 93 & 93 & 90 & 95 & 88 & 69 & 66 & 94 & 83 \\
\hline 08 & 81 & 86 & 87 & 88 & 97 & 88 & 98 & 68 & 71 & 87 & 85 \\
\hline 10 & 86 & 67 & 64 & 67 & 66 & 69 & 68 & 94 & 56 & 66 & 81 \\
\hline 11 & 72 & 65 & 66 & 64 & 69 & 67 & 72 & 56 & 84 & 66 & 58 \\
\hline 12 & 77 & 96 & 95 & 95 & 89 & 94 & 87 & 66 & 65 & 96 & 81 \\
\hline DS & 87 & 81 & 79 & 82 & 85 & 83 & 85 & 81 & 57 & 82 & - \\
\hline
\end{tabular}


Table B-180. South Dakota Site 1 Left, Medium Wavelengths.

\begin{tabular}{|c|c|c|c|c|c|c|c|c|c|c|c|}
\hline $\begin{array}{c}\text { Correlation } \\
\text { Reference }\end{array}$ & \multicolumn{10}{|c|}{ Correlated Device } \\
\hline 01 & 71 & 69 & 60 & 56 & 61 & 55 & 64 & 22 & 56 & 62 & 74 \\
\hline 02 & 60 & 77 & 60 & 56 & 67 & 57 & 68 & 18 & 57 & 62 & 78 \\
\hline 03 & 61 & 68 & 60 & 56 & 61 & 52 & 62 & 19 & 55 & 59 & 71 \\
\hline 04 & 60 & 56 & 58 & 56 & 57 & 52 & 58 & 18 & 51 & 57 & 58 \\
\hline 05 & 53 & 60 & 51 & 50 & 92 & 44 & 92 & 16 & 46 & 52 & 63 \\
\hline 06 & 61 & 57 & 56 & 52 & 49 & 61 & 52 & 24 & 56 & 54 & 55 \\
\hline 08 & 55 & 61 & 52 & 52 & 92 & 47 & 93 & 17 & 47 & 53 & 63 \\
\hline 10 & 26 & 22 & 21 & 20 & 18 & 25 & 19 & 86 & 22 & 20 & 24 \\
\hline 11 & 63 & 67 & 60 & 54 & 53 & 56 & 55 & 21 & 60 & 60 & 69 \\
\hline 12 & 63 & 62 & 60 & 57 & 58 & 54 & 60 & 17 & 56 & 60 & 65 \\
\hline DS & 64 & 78 & 63 & 58 & 69 & 55 & 70 & 20 & 58 & 65 & - \\
\hline
\end{tabular}

Table B-181. South Dakota Site 1 Left, Short Wavelengths.

\begin{tabular}{|c|c|c|c|c|c|c|c|c|c|c|c|}
\hline $\begin{array}{c}\text { Correlation } \\
\text { Reference }\end{array}$ & 01 & 02 & 03 & 04 & 05 & 06 & 08 & 10 & 11 & 12 & DS \\
\hline 01 & 37 & 27 & 22 & 15 & 30 & 14 & 33 & 8 & 19 & 18 & 26 \\
\hline 02 & 21 & 42 & 25 & 19 & 34 & 15 & 35 & 7 & 21 & 23 & 38 \\
\hline 03 & 22 & 30 & 27 & 16 & 28 & 12 & 29 & 7 & 19 & 18 & 32 \\
\hline 04 & 22 & 19 & 25 & 21 & 21 & 13 & 22 & 9 & 19 & 22 & 18 \\
\hline 05 & 17 & 22 & 18 & 14 & 66 & 8 & 63 & 5 & 15 & 15 & 24 \\
\hline 06 & 21 & 15 & 19 & 13 & 11 & 22 & 14 & 8 & 16 & 13 & 11 \\
\hline 08 & 20 & 22 & 18 & 14 & 63 & 10 & 73 & 5 & 15 & 15 & 20 \\
\hline 10 & 10 & 10 & 9 & 7 & 9 & 7 & 10 & 47 & 8 & 8 & 9 \\
\hline 11 & 18 & 24 & 18 & 11 & 21 & 9 & 21 & 7 & 20 & 14 & 23 \\
\hline 12 & 23 & 23 & 24 & 22 & 23 & 13 & 23 & 9 & 19 & 24 & 25 \\
\hline DS & 19 & 38 & 27 & 18 & 35 & 11 & 34 & 7 & 20 & 25 & - \\
\hline
\end{tabular}


Table B-182. South Dakota Site 1 Right, IRI Filter.

\begin{tabular}{|c|c|c|c|c|c|c|}
\hline Correlation & \multicolumn{6}{|c|}{ Correlated Device } \\
Reference & 01 & 05 & 08 & 10 & 11 & DS \\
\hline 01 & 83 & 79 & 82 & 40 & 63 & 78 \\
\hline 05 & 73 & $\mathbf{9 5}$ & 92 & 42 & 63 & 84 \\
\hline 08 & 77 & 92 & $\mathbf{9 5}$ & 42 & 65 & 79 \\
\hline 10 & 36 & 40 & 39 & 88 & 31 & 45 \\
\hline 11 & 66 & 68 & 71 & 34 & 70 & 64 \\
\hline DS & 72 & 82 & 78 & 44 & 60 & - \\
\hline
\end{tabular}

Table B-183. South Dakota Site 1 Right, Long Wavelengths.

\begin{tabular}{|c|c|c|c|c|c|c|}
\hline $\begin{array}{c}\text { Correlation } \\
\text { Reference }\end{array}$ & \multicolumn{6}{|c|}{ Correlated Device } \\
\hline 01 & 97 & 90 & 92 & 73 & 61 & 87 \\
\hline 05 & 90 & 99 & 97 & 64 & 67 & 83 \\
\hline 08 & 92 & 97 & 99 & 62 & 65 & 80 \\
\hline 10 & 73 & 64 & 62 & 94 & 57 & 85 \\
\hline 11 & 60 & 67 & 65 & 58 & 85 & 68 \\
\hline DS & 86 & 82 & 80 & 86 & 68 & - \\
\hline
\end{tabular}

Table B-184. South Dakota Site 1 Right, Medium Wavelengths.

\begin{tabular}{|c|c|c|c|c|c|c|}
\hline Correlation & \multicolumn{6}{|c|}{ Correlated Device } \\
Reference & 01 & 05 & 08 & 10 & 11 & DS \\
\hline 01 & 72 & 71 & 74 & 28 & 56 & 72 \\
\hline 05 & 61 & 92 & 90 & 27 & 57 & 75 \\
\hline 08 & 65 & 90 & 92 & 29 & 59 & 76 \\
\hline 10 & 27 & 32 & 33 & 83 & 26 & 34 \\
\hline 11 & 63 & 66 & 69 & 28 & 61 & 62 \\
\hline DS & 62 & 77 & 74 & 29 & 53 & - \\
\hline
\end{tabular}

Table B-185. South Dakota Site 1 Right, Short Wavelengths.

\begin{tabular}{|c|c|c|c|c|c|c|}
\hline Correlation & \multicolumn{6}{|c|}{ Correlated Device } \\
Reference & 01 & 05 & 08 & 10 & 11 & DS \\
\hline 01 & 40 & 29 & 34 & 8 & 23 & 23 \\
\hline 05 & 25 & 74 & 68 & 6 & 22 & 30 \\
\hline 08 & 28 & 68 & 77 & 6 & 23 & 25 \\
\hline 10 & 12 & 14 & 12 & 50 & 10 & 11 \\
\hline 11 & 21 & 22 & 25 & 6 & 19 & 17 \\
\hline DS & 22 & 31 & 28 & 5 & 18 & - \\
\hline
\end{tabular}


Table B-186. South Dakota Site 2 Left, IRI Filter.

\begin{tabular}{|c|c|c|c|c|c|c|c|c|c|c|c|}
\hline Correlation & \multicolumn{11}{|c|}{ Correlated Device } \\
\hline Reference & 01 & 02 & 03 & 04 & 05 & 06 & 08 & 10 & 11 & 12 & DS \\
\hline 01 & 30 & 24 & 20 & 25 & 21 & 25 & 24 & 18 & 23 & 21 & 24 \\
\hline 02 & 21 & 49 & 15 & 22 & 46 & 20 & 51 & 28 & 20 & 18 & 50 \\
\hline 03 & 21 & 18 & 16 & 20 & 15 & 19 & 17 & 12 & 19 & 18 & 16 \\
\hline 04 & 22 & 22 & 18 & 25 & 21 & 21 & 23 & 15 & 22 & 19 & 21 \\
\hline 05 & 19 & 43 & 14 & 19 & 91 & 17 & 88 & 32 & 16 & 14 & 75 \\
\hline 06 & 25 & 20 & 18 & 21 & 18 & 23 & 21 & 15 & 23 & 20 & 19 \\
\hline 08 & 21 & 48 & 15 & 22 & 88 & 19 & 93 & 33 & 18 & 15 & 78 \\
\hline 10 & 16 & 29 & 11 & 14 & 34 & 14 & 35 & 71 & 14 & 12 & 33 \\
\hline 11 & 22 & 24 & 17 & 23 & 18 & 22 & 21 & 15 & 21 & 20 & 22 \\
\hline 12 & 22 & 18 & 18 & 19 & 15 & 20 & 16 & 12 & 21 & 19 & 16 \\
\hline DS & 20 & 50 & 14 & 21 & 75 & 19 & 76 & 30 & 19 & 16 & - \\
\hline
\end{tabular}

Table B-187. South Dakota Site 2 Left, Long Wavelengths.

\begin{tabular}{|c|c|c|c|c|c|c|c|c|c|c|c|}
\hline $\begin{array}{c}\text { Correlation } \\
\text { Reference }\end{array}$ & \multicolumn{10}{|c|}{ Correlated Device } \\
\hline 01 & 89 & 64 & 61 & 65 & 76 & 67 & 76 & 72 & 59 & 63 & 73 \\
\hline 02 & 64 & 94 & 81 & 86 & 80 & 82 & 80 & 68 & 66 & 83 & 83 \\
\hline 03 & 60 & 82 & 75 & 78 & 77 & 76 & 78 & 63 & 61 & 76 & 76 \\
\hline 04 & 64 & 86 & 78 & 83 & 80 & 80 & 80 & 68 & 62 & 79 & 82 \\
\hline 05 & 76 & 80 & 78 & 80 & 98 & 83 & 98 & 75 & 73 & 78 & 81 \\
\hline 06 & 67 & 82 & 77 & 80 & 83 & 83 & 83 & 71 & 69 & 79 & 82 \\
\hline 08 & 76 & 81 & 78 & 80 & 98 & 83 & 99 & 76 & 72 & 78 & 81 \\
\hline 10 & 72 & 68 & 63 & 67 & 75 & 70 & 75 & 86 & 57 & 65 & 82 \\
\hline 11 & 59 & 66 & 61 & 63 & 72 & 69 & 72 & 57 & 74 & 64 & 59 \\
\hline 12 & 62 & 83 & 76 & 79 & 78 & 79 & 78 & 65 & 64 & 77 & 79 \\
\hline DS & 72 & 82 & 75 & 82 & 81 & 82 & 81 & 82 & 59 & 79 & - \\
\hline
\end{tabular}


Table B-188. South Dakota Site 2 Left, Medium Wavelengths.

\begin{tabular}{|c|c|c|c|c|c|c|c|c|c|c|c|}
\hline $\begin{array}{c}\text { Correlation } \\
\text { Reference }\end{array}$ & \multicolumn{10}{|c|}{ Correlated Device } \\
\hline 01 & 19 & 17 & 12 & 15 & 11 & 15 & 14 & 9 & 14 & 13 & 14 \\
\hline 02 & 13 & 36 & 9 & 14 & 29 & 13 & 34 & 16 & 13 & 12 & 34 \\
\hline 03 & 14 & 13 & 11 & 13 & 9 & 13 & 10 & 7 & 13 & 13 & 10 \\
\hline 04 & 14 & 14 & 11 & 17 & 11 & 14 & 13 & 8 & 15 & 14 & 12 \\
\hline 05 & 9 & 25 & 7 & 9 & 81 & 9 & 78 & 16 & 9 & 7 & 59 \\
\hline 06 & 16 & 13 & 12 & 14 & 10 & 17 & 12 & 9 & 15 & 14 & 11 \\
\hline 08 & 11 & 29 & 8 & 11 & 78 & 11 & 87 & 17 & 11 & 8 & 71 \\
\hline 10 & 8 & 15 & 6 & 7 & 18 & 8 & 19 & 59 & 8 & 6 & 17 \\
\hline 11 & 14 & 17 & 12 & 16 & 11 & 15 & 13 & 9 & 14 & 14 & 15 \\
\hline 12 & 16 & 12 & 12 & 14 & 8 & 14 & 10 & 8 & 16 & 15 & 9 \\
\hline DS & 11 & 34 & 8 & 12 & 65 & 11 & 72 & 14 & 11 & 9 & - \\
\hline
\end{tabular}

Table B-189. South Dakota Site 2 Left, Short Wavelengths.

\begin{tabular}{|c|c|c|c|c|c|c|c|c|c|c|c|}
\hline $\begin{array}{c}\text { Correlation } \\
\text { Reference }\end{array}$ & \multicolumn{10}{|c|}{ Correlated Device } \\
\hline 01 & 13 & 8 & 7 & 7 & 4 & 8 & 5 & 6 & 8 & 6 & 4 \\
\hline 02 & 4 & 15 & 4 & 7 & 9 & 5 & 11 & 7 & 4 & 7 & 10 \\
\hline 03 & 8 & 7 & 9 & 7 & 3 & 6 & 4 & 6 & 8 & 7 & 4 \\
\hline 04 & 7 & 7 & 7 & 11 & 4 & 9 & 4 & 5 & 8 & 10 & 3 \\
\hline 05 & 2 & 6 & 2 & 2 & 46 & 2 & 37 & 3 & 2 & 2 & 23 \\
\hline 06 & 9 & 5 & 6 & 9 & 3 & 11 & 3 & 5 & 8 & 9 & 2 \\
\hline 08 & 3 & 7 & 2 & 3 & 37 & 2 & 57 & 3 & 2 & 3 & 31 \\
\hline 10 & 3 & 7 & 3 & 4 & 6 & 3 & 6 & 19 & 4 & 3 & 5 \\
\hline 11 & 7 & 8 & 6 & 6 & 3 & 6 & 4 & 7 & 9 & 6 & 4 \\
\hline 12 & 9 & 7 & 8 & 10 & 3 & 9 & 4 & 5 & 9 & 12 & 3 \\
\hline DS & 2 & 10 & 2 & 3 & 13 & 2 & 23 & 2 & 2 & 3 & - \\
\hline
\end{tabular}


Table B-190. South Dakota Site 2 Right, IRI Filter.

\begin{tabular}{|c|c|c|c|c|c|c|}
\hline Correlation & \multicolumn{6}{|c|}{ Correlated Device } \\
Reference & 01 & 05 & 08 & 10 & 11 & DS \\
\hline 01 & 47 & 37 & 36 & 24 & 39 & 39 \\
\hline 05 & 34 & $\mathbf{9 4}$ & 93 & 45 & 38 & 84 \\
\hline 08 & 33 & 93 & 93 & 46 & 37 & 83 \\
\hline 10 & 23 & 47 & 48 & 84 & 20 & 54 \\
\hline 11 & 35 & 41 & 40 & 22 & 41 & 39 \\
\hline DS & 35 & 86 & 85 & 51 & 34 & - \\
\hline
\end{tabular}

Table B-191. South Dakota Site 2 Right, Long Wavelengths.

\begin{tabular}{|c|c|c|c|c|c|c|}
\hline Correlation & \multicolumn{6}{|c|}{ Correlated Device } \\
Reference & 01 & 05 & 08 & 10 & 11 & DS \\
\hline 01 & 93 & 75 & 76 & 70 & 66 & 80 \\
\hline 05 & 75 & 98 & 98 & 79 & 85 & 88 \\
\hline 08 & 76 & 98 & 98 & 79 & 85 & 89 \\
\hline 10 & 69 & 79 & 79 & 93 & 67 & 86 \\
\hline 11 & 66 & 85 & 85 & 67 & 87 & 77 \\
\hline DS & 80 & 88 & 89 & 86 & 77 & - \\
\hline
\end{tabular}

Table B-192. South Dakota Site 2 Right, Medium Wavelengths.

\begin{tabular}{|c|c|c|c|c|c|c|}
\hline Correlation & \multicolumn{6}{|c|}{ Correlated Device } \\
Reference & 01 & 05 & 08 & 10 & 11 & DS \\
\hline 01 & 28 & 23 & 22 & 11 & 27 & 23 \\
\hline 05 & 19 & 90 & 87 & 25 & 22 & 78 \\
\hline 08 & 17 & 87 & 88 & 26 & 20 & 74 \\
\hline 10 & 10 & 28 & 29 & 72 & 10 & 29 \\
\hline 11 & 23 & 27 & 25 & 12 & 27 & 26 \\
\hline DS & 18 & 83 & 80 & 26 & 20 & - \\
\hline
\end{tabular}

Table B-193. South Dakota Site 2 Right, Short Wavelengths.

\begin{tabular}{|c|c|c|c|c|c|c|}
\hline $\begin{array}{c}\text { Correlation } \\
\text { Reference }\end{array}$ & 01 & 05 & 08 & 10 & 11 & DS \\
\hline 01 & 12 & 5 & 5 & 5 & 8 & 5 \\
\hline 05 & 3 & 59 & 53 & 3 & 3 & 37 \\
\hline 08 & 3 & 53 & 57 & 3 & 3 & 32 \\
\hline 10 & 3 & 7 & 7 & 17 & 3 & 6 \\
\hline 11 & 6 & 5 & 4 & 5 & 10 & 4 \\
\hline DS & 3 & 26 & 26 & 3 & 2 & - \\
\hline
\end{tabular}


Table B-194. South Dakota Site 3 Left, IRI Filter.

\begin{tabular}{|c|c|c|c|c|c|c|c|c|c|c|c|}
\hline $\begin{array}{c}\text { Correlation } \\
\text { Reference }\end{array}$ & 01 & 02 & 03 & 04 & 05 & 06 & 08 & 10 & 11 & 12 & DS \\
\hline 01 & 42 & 40 & 30 & 24 & 40 & 25 & 44 & 30 & 32 & 26 & 39 \\
\hline 02 & 35 & 64 & 29 & 22 & 69 & 23 & 74 & 31 & 31 & 24 & 55 \\
\hline 03 & 32 & 34 & 29 & 24 & 35 & 23 & 37 & 22 & 30 & 26 & 32 \\
\hline 04 & 28 & 22 & 27 & 29 & 26 & 24 & 27 & 18 & 26 & 27 & 22 \\
\hline 05 & 37 & 66 & 31 & 24 & 91 & 22 & 91 & 38 & 32 & 27 & 62 \\
\hline 06 & 29 & 23 & 26 & 24 & 24 & 28 & 26 & 17 & 28 & 24 & 19 \\
\hline 08 & 40 & 71 & 33 & 25 & 91 & 24 & $\mathbf{9 5}$ & 37 & 34 & 27 & 65 \\
\hline 10 & 28 & 34 & 20 & 17 & 40 & 16 & 39 & 73 & 22 & 18 & 36 \\
\hline 11 & 33 & 36 & 29 & 22 & 36 & 24 & 39 & 23 & 32 & 24 & 34 \\
\hline 12 & 30 & 24 & 29 & 27 & 29 & 24 & 30 & 19 & 27 & 28 & 24 \\
\hline DS & 34 & 55 & 27 & 22 & 67 & 19 & 70 & 33 & 29 & 24 & - \\
\hline
\end{tabular}

Table B-195. South Dakota Site 3 Left, Long Wavelengths.

\begin{tabular}{|c|c|c|c|c|c|c|c|c|c|c|c|}
\hline $\begin{array}{c}\text { Correlation } \\
\text { Reference }\end{array}$ & \multicolumn{10}{|c|}{ Correlated Device } \\
\hline 01 & 94 & 70 & 63 & 72 & 76 & 69 & 77 & 83 & 51 & 68 & 77 \\
\hline 02 & 70 & 97 & 92 & 90 & 83 & 93 & 82 & 59 & 80 & 93 & 69 \\
\hline 03 & 63 & 92 & 91 & 85 & 78 & 87 & 78 & 51 & 81 & 88 & 60 \\
\hline 04 & 72 & 90 & 86 & 90 & 85 & 89 & 84 & 61 & 73 & 88 & 70 \\
\hline 05 & 76 & 83 & 79 & 85 & 98 & 81 & 98 & 63 & 67 & 81 & 60 \\
\hline 06 & 69 & 93 & 88 & 89 & 81 & 91 & 81 & 58 & 78 & 91 & 68 \\
\hline 08 & 77 & 82 & 78 & 85 & 98 & 81 & 99 & 62 & 67 & 80 & 59 \\
\hline 10 & 83 & 58 & 51 & 61 & 63 & 58 & 62 & 93 & 40 & 57 & 80 \\
\hline 11 & 51 & 80 & 81 & 72 & 67 & 77 & 67 & 40 & 83 & 77 & 48 \\
\hline 12 & 68 & 93 & 88 & 88 & 81 & 91 & 80 & 57 & 78 & 90 & 67 \\
\hline DS & 76 & 69 & 61 & 70 & 60 & 68 & 60 & 80 & 48 & 68 & - \\
\hline
\end{tabular}


Table B-196. South Dakota Site 3 Left, Medium Wavelengths.

\begin{tabular}{|c|c|c|c|c|c|c|c|c|c|c|c|}
\hline $\begin{array}{c}\text { Correlation } \\
\text { Reference }\end{array}$ & \multicolumn{10}{|c|}{ Correlated Device } \\
\hline 01 & 29 & 31 & 21 & 15 & 28 & 16 & 33 & 18 & 22 & 17 & 27 \\
\hline 02 & 23 & 54 & 20 & 15 & 57 & 16 & 64 & 22 & 22 & 17 & 46 \\
\hline 03 & 24 & 27 & 22 & 18 & 26 & 16 & 29 & 15 & 23 & 19 & 24 \\
\hline 04 & 20 & 15 & 21 & 21 & 19 & 17 & 20 & 11 & 20 & 19 & 14 \\
\hline 05 & 23 & 52 & 21 & 16 & 86 & 13 & 85 & 25 & 22 & 18 & 54 \\
\hline 06 & 21 & 16 & 19 & 17 & 16 & 21 & 18 & 11 & 21 & 17 & 12 \\
\hline 08 & 27 & 57 & 23 & 17 & 85 & 15 & 92 & 25 & 24 & 19 & 56 \\
\hline 10 & 15 & 25 & 13 & 9 & 29 & 9 & 29 & 60 & 14 & 11 & 23 \\
\hline 11 & 24 & 29 & 22 & 15 & 28 & 17 & 30 & 17 & 24 & 17 & 26 \\
\hline 12 & 21 & 17 & 22 & 19 & 21 & 17 & 22 & 13 & 21 & 21 & 16 \\
\hline DS & 21 & 46 & 18 & 14 & 61 & 12 & 64 & 19 & 20 & 16 & - \\
\hline
\end{tabular}

Table B-197. South Dakota Site 3 Left, Short Wavelengths.

\begin{tabular}{|c|c|c|c|c|c|c|c|c|c|c|c|}
\hline $\begin{array}{c}\text { Correlation } \\
\text { Reference }\end{array}$ & \multicolumn{10}{|c|}{ Correlated Device } \\
\hline 01 & 15 & 12 & 8 & 4 & 6 & 5 & 10 & 9 & 8 & 5 & 9 \\
\hline 02 & 6 & 27 & 6 & 5 & 20 & 4 & 31 & 5 & 5 & 5 & 20 \\
\hline 03 & 10 & 11 & 11 & 6 & 9 & 5 & 11 & 8 & 9 & 6 & 8 \\
\hline 04 & 10 & 5 & 11 & 12 & 5 & 9 & 6 & 6 & 9 & 10 & 4 \\
\hline 05 & 4 & 15 & 5 & 4 & 53 & 2 & 49 & 4 & 5 & 4 & 19 \\
\hline 06 & 11 & 4 & 10 & 9 & 3 & 12 & 4 & 6 & 9 & 9 & 3 \\
\hline 08 & 6 & 21 & 7 & 4 & 49 & 3 & 69 & 4 & 5 & 5 & 23 \\
\hline 10 & 6 & 10 & 5 & 4 & 8 & 4 & 8 & 20 & 6 & 4 & 9 \\
\hline 11 & 8 & 9 & 8 & 4 & 8 & 5 & 9 & 8 & 10 & 6 & 7 \\
\hline 12 & 10 & 5 & 10 & 10 & 6 & 9 & 7 & 6 & 10 & 11 & 4 \\
\hline DS & 4 & 20 & 4 & 4 & 24 & 3 & 37 & 4 & 4 & 4 & - \\
\hline
\end{tabular}


Table B-198. South Dakota Site 3 Right, IRI Filter.

\begin{tabular}{|c|c|c|c|c|c|c|}
\hline $\begin{array}{c}\text { Correlation } \\
\text { Reference }\end{array}$ & \multicolumn{6}{|c|}{ Correlated Device } \\
\hline 01 & 45 & 41 & 40 & 24 & 35 & 41 \\
\hline 05 & 37 & 92 & 91 & 40 & 33 & 76 \\
\hline 08 & 36 & 91 & $\mathbf{9 6}$ & 41 & 32 & 72 \\
\hline 10 & 22 & 42 & 43 & 80 & 19 & 45 \\
\hline 11 & 35 & 37 & 36 & 21 & 33 & 36 \\
\hline DS & 36 & 81 & 77 & 45 & 31 & - \\
\hline
\end{tabular}

Table B-199. South Dakota Site 3 Right, Long Wavelengths.

\begin{tabular}{|c|c|c|c|c|c|c|}
\hline Correlation & \multicolumn{6}{|c|}{ Correlated Device } \\
Reference & 01 & 05 & 08 & 10 & 11 & DS \\
\hline 01 & 92 & 75 & 77 & 81 & 73 & 86 \\
\hline 05 & 74 & 99 & 97 & 57 & 85 & 66 \\
\hline 08 & 76 & 97 & 99 & 58 & 85 & 68 \\
\hline 10 & 80 & 56 & 58 & 90 & 57 & 84 \\
\hline 11 & 73 & 85 & 85 & 57 & 84 & 65 \\
\hline DS & 85 & 67 & 68 & 84 & 65 & - \\
\hline
\end{tabular}

Table B-200. South Dakota Site 3 Right, Medium Wavelengths.

\begin{tabular}{|c|c|c|c|c|c|c|}
\hline Correlation & \multicolumn{6}{|c|}{ Correlated Device } \\
Reference & 01 & 05 & 08 & 10 & 11 & DS \\
\hline 01 & 34 & 33 & 32 & 16 & 26 & 32 \\
\hline 05 & 27 & 90 & 87 & 30 & 23 & 76 \\
\hline 08 & 26 & 87 & 93 & 30 & 22 & 70 \\
\hline 10 & 13 & 33 & 34 & 71 & 13 & 34 \\
\hline 11 & 26 & 29 & 28 & 15 & 24 & 29 \\
\hline DS & 25 & 81 & 78 & 32 & 23 & - \\
\hline
\end{tabular}

Table B-201. South Dakota Site 3 Right, Short Wavelengths.

\begin{tabular}{|c|c|c|c|c|c|c|}
\hline $\begin{array}{c}\text { Correlation } \\
\text { Reference }\end{array}$ & 01 & 05 & 08 & 10 & 11 & DS \\
\hline 01 & 17 & 7 & 10 & 6 & 8 & 9 \\
\hline 05 & 5 & 63 & 60 & 6 & 5 & 38 \\
\hline 08 & 6 & 60 & 72 & 7 & 5 & 39 \\
\hline 10 & 4 & 13 & 15 & 25 & 4 & 12 \\
\hline 11 & 7 & 7 & 8 & 7 & 12 & 8 \\
\hline DS & 5 & 35 & 38 & 5 & 4 & - \\
\hline
\end{tabular}


Table B-202. South Dakota Site 4 Left, IRI Filter.

\begin{tabular}{|c|c|c|c|c|c|c|c|c|c|c|c|}
\hline Correlation & \multicolumn{11}{|c|}{ Correlated Device } \\
\hline Reference & 01 & 02 & 03 & 04 & 05 & 06 & 08 & 10 & 11 & 12 & DS \\
\hline 01 & 30 & 26 & 22 & 22 & 25 & 19 & 27 & 19 & 21 & 20 & 27 \\
\hline 02 & 23 & 36 & 20 & 19 & 40 & 18 & 42 & 24 & 21 & 21 & 38 \\
\hline 03 & 23 & 23 & 20 & 19 & 22 & 19 & 24 & 13 & 21 & 20 & 23 \\
\hline 04 & 26 & 19 & 21 & 23 & 20 & 19 & 21 & 12 & 24 & 20 & 19 \\
\hline 05 & 22 & 37 & 20 & 18 & 89 & 16 & 89 & 27 & 18 & 18 & 74 \\
\hline 06 & 21 & 18 & 19 & 19 & 18 & 20 & 18 & 14 & 20 & 20 & 16 \\
\hline 08 & 24 & 39 & 21 & 19 & 89 & 17 & 92 & 28 & 19 & 19 & 77 \\
\hline 10 & 17 & 24 & 12 & 12 & 29 & 14 & 30 & 62 & 13 & 13 & 33 \\
\hline 11 & 23 & 25 & 21 & 21 & 21 & 19 & 22 & 14 & 29 & 22 & 23 \\
\hline 12 & 22 & 21 & 21 & 20 & 20 & 20 & 21 & 14 & 23 & 23 & 19 \\
\hline DS & 24 & 38 & 20 & 19 & 80 & 16 & 81 & 29 & 20 & 19 & - \\
\hline
\end{tabular}

Table B-203. South Dakota Site 4 Left, Long Wavelengths.

\begin{tabular}{|c|c|c|c|c|c|c|c|c|c|c|c|}
\hline $\begin{array}{c}\text { Correlation } \\
\text { Reference }\end{array}$ & \multicolumn{10}{|c|}{ Correlated Device } \\
\hline 01 & 92 & 67 & 62 & 68 & 69 & 69 & 70 & 79 & 53 & 69 & 88 \\
\hline 02 & 67 & 95 & 89 & 89 & 86 & 85 & 85 & 56 & 80 & 89 & 64 \\
\hline 03 & 62 & 89 & 86 & 85 & 82 & 82 & 82 & 50 & 76 & 85 & 57 \\
\hline 04 & 68 & 89 & 86 & 88 & 86 & 86 & 85 & 57 & 75 & 88 & 65 \\
\hline 05 & 69 & 86 & 82 & 86 & 99 & 87 & 98 & 56 & 73 & 86 & 63 \\
\hline 06 & 69 & 85 & 83 & 86 & 87 & 87 & 86 & 57 & 75 & 88 & 66 \\
\hline 08 & 70 & 85 & 82 & 85 & 98 & 86 & 98 & 56 & 72 & 85 & 63 \\
\hline 10 & 79 & 56 & 49 & 57 & 54 & 57 & 54 & 89 & 49 & 57 & 89 \\
\hline 11 & 52 & 80 & 76 & 74 & 73 & 75 & 72 & 48 & 80 & 76 & 49 \\
\hline 12 & 69 & 89 & 85 & 88 & 86 & 88 & 85 & 58 & 77 & 90 & 66 \\
\hline DS & 87 & 63 & 57 & 65 & 62 & 65 & 63 & 89 & 51 & 65 & - \\
\hline
\end{tabular}


Table B-204. South Dakota Site 4 Left, Medium Wavelengths.

\begin{tabular}{|c|c|c|c|c|c|c|c|c|c|c|c|}
\hline $\begin{array}{c}\text { Correlation } \\
\text { Reference }\end{array}$ & \multicolumn{10}{|c|}{ Correlated Device } \\
\hline 01 & 20 & 20 & 15 & 15 & 18 & 12 & 20 & 11 & 15 & 13 & 19 \\
\hline 02 & 15 & 29 & 14 & 14 & 30 & 12 & 32 & 15 & 15 & 15 & 28 \\
\hline 03 & 17 & 18 & 15 & 14 & 17 & 13 & 18 & 9 & 16 & 15 & 18 \\
\hline 04 & 19 & 14 & 16 & 18 & 14 & 13 & 15 & 8 & 18 & 15 & 14 \\
\hline 05 & 14 & 26 & 13 & 12 & 84 & 10 & 85 & 14 & 12 & 11 & 64 \\
\hline 06 & 14 & 12 & 13 & 13 & 12 & 14 & 12 & 9 & 14 & 15 & 10 \\
\hline 08 & 15 & 27 & 14 & 12 & 85 & 11 & 89 & 15 & 13 & 12 & 68 \\
\hline 10 & 9 & 13 & 7 & 6 & 18 & 8 & 18 & 51 & 8 & 7 & 18 \\
\hline 11 & 17 & 19 & 15 & 15 & 16 & 13 & 17 & 10 & 20 & 15 & 19 \\
\hline 12 & 16 & 15 & 15 & 15 & 14 & 15 & 15 & 9 & 17 & 18 & 13 \\
\hline DS & 15 & 28 & 13 & 14 & 76 & 10 & 77 & 14 & 14 & 13 & - \\
\hline
\end{tabular}

Table B-205. South Dakota Site 4 Left, Short Wavelengths.

\begin{tabular}{|c|c|c|c|c|c|c|c|c|c|c|c|}
\hline $\begin{array}{c}\text { Correlation } \\
\text { Reference }\end{array}$ & \multicolumn{10}{|c|}{ Correlated Device } \\
\hline 01 & 12 & 02 & 03 & 04 & 05 & 06 & 08 & 10 & 11 & 12 & DS \\
\hline 02 & 6 & 16 & 6 & 6 & 9 & 6 & 10 & 8 & 9 & 6 & 10 \\
\hline 03 & 8 & 10 & 10 & 6 & 8 & 6 & 9 & 7 & 9 & 6 & 9 \\
\hline 04 & 9 & 6 & 9 & 10 & 6 & 9 & 6 & 6 & 10 & 9 & 6 \\
\hline 05 & 5 & 13 & 5 & 3 & 60 & 3 & 62 & 4 & 5 & 4 & 30 \\
\hline 06 & 8 & 6 & 7 & 9 & 5 & 10 & 5 & 7 & 8 & 10 & 4 \\
\hline 08 & 5 & 13 & 5 & 3 & 62 & 3 & 67 & 4 & 5 & 4 & 34 \\
\hline 10 & 5 & 7 & 5 & 4 & 7 & 4 & 8 & 23 & 6 & 4 & 9 \\
\hline 11 & 8 & 10 & 8 & 6 & 8 & 6 & 8 & 9 & 10 & 6 & 14 \\
\hline 12 & 9 & 8 & 9 & 10 & 6 & 10 & 6 & 6 & 10 & 13 & 6 \\
\hline DS & 5 & 16 & 5 & 6 & 44 & 4 & 49 & 5 & 8 & 6 & - \\
\hline
\end{tabular}


Table B-206. South Dakota Site 4 Right, IRI Filter.

\begin{tabular}{|c|c|c|c|c|c|c|}
\hline Correlation & \multicolumn{6}{|c|}{ Correlated Device } \\
Reference & 01 & 05 & 08 & 10 & 11 & DS \\
\hline 01 & 36 & 32 & 32 & 17 & 30 & 34 \\
\hline 05 & 28 & 91 & 91 & 29 & 29 & 73 \\
\hline 08 & 29 & 91 & 91 & 29 & 29 & 73 \\
\hline 10 & 16 & 31 & 31 & 61 & 13 & 41 \\
\hline 11 & 27 & 33 & 33 & 14 & 30 & 31 \\
\hline DS & 30 & 79 & 80 & 38 & 27 & - \\
\hline
\end{tabular}

Table B-207. South Dakota Site 4 Right, Long Wavelengths.

\begin{tabular}{|c|c|c|c|c|c|c|}
\hline Correlation & \multicolumn{6}{|c|}{ Correlated Device } \\
Reference & 01 & 05 & 08 & 10 & 11 & DS \\
\hline 01 & 89 & 68 & 70 & 77 & 57 & 83 \\
\hline 05 & 68 & 98 & 97 & 52 & 78 & 64 \\
\hline 08 & 70 & 97 & 99 & 52 & 76 & 63 \\
\hline 10 & 77 & 52 & 51 & 87 & 46 & 86 \\
\hline 11 & 57 & 78 & 76 & 47 & 78 & 54 \\
\hline DS & 82 & 64 & 63 & 86 & 53 & - \\
\hline
\end{tabular}

Table B-208. South Dakota Site 4 Right, Medium Wavelengths.

\begin{tabular}{|c|c|c|c|c|c|c|}
\hline Correlation & \multicolumn{6}{|c|}{ Correlated Device } \\
Reference & 01 & 05 & 08 & 10 & 11 & DS \\
\hline 01 & 26 & 26 & 26 & 9 & 23 & 26 \\
\hline 05 & 20 & 87 & 88 & 17 & 22 & 66 \\
\hline 08 & 20 & 88 & 88 & 16 & 22 & 67 \\
\hline 10 & 7 & 20 & 19 & 51 & 8 & 28 \\
\hline 11 & 20 & 28 & 28 & 10 & 22 & 26 \\
\hline DS & 20 & 72 & 74 & 23 & 20 & - \\
\hline
\end{tabular}

Table B-209. South Dakota Site 4 Right, Short Wavelengths.

\begin{tabular}{|c|c|c|c|c|c|c|}
\hline Correlation & \multicolumn{6}{|c|}{ Correlated Device } \\
Reference & 01 & 05 & 08 & 10 & 11 & DS \\
\hline 01 & 15 & 13 & 13 & 7 & 11 & 12 \\
\hline 05 & 8 & 71 & 69 & 5 & 8 & 28 \\
\hline 08 & 8 & 69 & 72 & 5 & 9 & 29 \\
\hline 10 & 4 & 10 & 10 & 28 & 5 & 9 \\
\hline 11 & 7 & 15 & 15 & 8 & 11 & 10 \\
\hline DS & 7 & 26 & 28 & 4 & 7 & - \\
\hline
\end{tabular}


Table B-210. South Dakota Site 5 Left, IRI Filter.

\begin{tabular}{|c|c|c|c|c|c|c|c|c|c|c|c|}
\hline $\begin{array}{c}\text { Correlation } \\
\text { Reference }\end{array}$ & \multicolumn{10}{|c|}{ Correlated Device } \\
\hline 01 & 76 & 68 & 66 & 51 & 80 & 63 & 75 & 29 & 63 & 64 & 77 \\
\hline 02 & 70 & 69 & 64 & 51 & 73 & 61 & 70 & 24 & 62 & 65 & 72 \\
\hline 03 & 71 & 68 & 73 & 61 & 75 & 72 & 78 & 27 & 72 & 76 & 73 \\
\hline 04 & 57 & 51 & 65 & 58 & 56 & 64 & 65 & 25 & 67 & 68 & 54 \\
\hline 05 & 76 & 68 & 68 & 51 & 92 & 63 & 85 & 29 & 64 & 65 & 74 \\
\hline 06 & 69 & 61 & 72 & 64 & 68 & 83 & 77 & 41 & 81 & 79 & 62 \\
\hline 08 & 79 & 72 & 76 & 61 & 85 & 73 & $\mathbf{9 5}$ & 30 & 73 & 77 & 77 \\
\hline 10 & 32 & 27 & 28 & 25 & 31 & 40 & 32 & 77 & 39 & 30 & 26 \\
\hline 11 & 68 & 67 & 71 & 61 & 68 & 78 & 78 & 38 & 84 & 78 & 66 \\
\hline 12 & 70 & 65 & 76 & 68 & 70 & 79 & 82 & 30 & 78 & 84 & 69 \\
\hline DS & 74 & 72 & 67 & 54 & 78 & 62 & 75 & 24 & 61 & 69 & - \\
\hline
\end{tabular}

Table B-211. South Dakota Site 5 Left, Long Wavelengths.

\begin{tabular}{|c|c|c|c|c|c|c|c|c|c|c|c|}
\hline $\begin{array}{c}\text { Correlation } \\
\text { Reference }\end{array}$ & \multicolumn{10}{|c|}{ Correlated Device } \\
\hline 01 & 91 & 81 & 80 & 83 & 81 & 87 & 87 & 76 & 70 & 86 & 88 \\
\hline 02 & 80 & 93 & 91 & 80 & 92 & 82 & 85 & 63 & 65 & 84 & 75 \\
\hline 03 & 79 & 92 & 95 & 83 & 93 & 84 & 88 & 60 & 66 & 88 & 74 \\
\hline 04 & 84 & 80 & 85 & 88 & 82 & 90 & 90 & 65 & 71 & 91 & 80 \\
\hline 05 & 80 & 92 & 93 & 81 & 99 & 83 & 91 & 63 & 65 & 85 & 74 \\
\hline 06 & 87 & 82 & 85 & 90 & 84 & 94 & 92 & 72 & 75 & 93 & 83 \\
\hline 08 & 86 & 86 & 89 & 89 & 91 & 90 & 98 & 66 & 71 & 94 & 80 \\
\hline 10 & 76 & 63 & 61 & 65 & 63 & 72 & 67 & 86 & 60 & 66 & 73 \\
\hline 11 & 71 & 66 & 67 & 72 & 66 & 76 & 72 & 61 & 83 & 74 & 66 \\
\hline 12 & 86 & 84 & 89 & 91 & 86 & 93 & 94 & 66 & 73 & 96 & 83 \\
\hline DS & 88 & 75 & 74 & 80 & 75 & 83 & 81 & 72 & 65 & 83 & - \\
\hline
\end{tabular}


Table B-212. South Dakota Site 5 Left, Medium Wavelengths.

\begin{tabular}{|c|c|c|c|c|c|c|c|c|c|c|c|}
\hline $\begin{array}{c}\text { Correlation } \\
\text { Reference }\end{array}$ & \multicolumn{10}{|c|}{ Correlated Device } \\
\hline 01 & 73 & 61 & 61 & 44 & 74 & 58 & 73 & 26 & 61 & 59 & 74 \\
\hline 02 & 67 & 64 & 61 & 46 & 68 & 57 & 70 & 24 & 62 & 61 & 66 \\
\hline 03 & 69 & 66 & 71 & 54 & 72 & 67 & 78 & 28 & 72 & 73 & 71 \\
\hline 04 & 52 & 46 & 62 & 52 & 51 & 58 & 59 & 25 & 64 & 62 & 48 \\
\hline 05 & 73 & 61 & 63 & 45 & 86 & 58 & 84 & 27 & 62 & 61 & 73 \\
\hline 06 & 65 & 57 & 70 & 57 & 64 & 79 & 73 & 40 & 80 & 75 & 57 \\
\hline 08 & 78 & 68 & 72 & 54 & 84 & 67 & 95 & 29 & 72 & 72 & 77 \\
\hline 10 & 30 & 27 & 30 & 24 & 30 & 39 & 33 & 72 & 41 & 31 & 26 \\
\hline 11 & 68 & 67 & 71 & 55 & 68 & 74 & 79 & 38 & 84 & 74 & 66 \\
\hline 12 & 67 & 62 & 74 & 62 & 67 & 75 & 78 & 31 & 79 & 82 & 65 \\
\hline DS & 70 & 66 & 62 & 48 & 71 & 57 & 74 & 23 & 60 & 65 & - \\
\hline
\end{tabular}

Table B-213. South Dakota Site 5 Left, Short Wavelengths.

\begin{tabular}{|c|c|c|c|c|c|c|c|c|c|c|c|}
\hline Correlation & \multicolumn{11}{|c|}{ Correlated Device } \\
\hline Reference & 01 & 02 & 03 & 04 & 05 & 06 & 08 & 10 & 11 & 12 & DS \\
\hline 01 & 38 & 15 & 29 & 9 & 20 & 18 & 32 & 4 & 32 & 19 & 27 \\
\hline 02 & 27 & 25 & 31 & 13 & 19 & 16 & 26 & 5 & 30 & 23 & 21 \\
\hline 03 & 32 & 19 & 40 & 11 & 19 & 19 & 35 & 4 & 34 & 22 & 31 \\
\hline 04 & 18 & 13 & 24 & 11 & 14 & 11 & 17 & 6 & 20 & 16 & 13 \\
\hline 05 & 37 & 17 & 33 & 10 & 38 & 18 & 36 & 5 & 32 & 23 & 28 \\
\hline 06 & 29 & 16 & 28 & 12 & 16 & 28 & 25 & 5 & 36 & 18 & 12 \\
\hline 08 & 33 & 18 & 33 & 13 & 36 & 19 & 66 & 4 & 34 & 27 & 39 \\
\hline 10 & 9 & 8 & 9 & 7 & 9 & 9 & 8 & 19 & 9 & 8 & 6 \\
\hline 11 & 24 & 14 & 24 & 8 & 12 & 17 & 22 & 3 & 40 & 17 & 24 \\
\hline 12 & 28 & 23 & 31 & 16 & 20 & 18 & 37 & 4 & 34 & 30 & 30 \\
\hline DS & 22 & 21 & 24 & 13 & 14 & 12 & 32 & 3 & 26 & 30 & - \\
\hline
\end{tabular}


Table B-214. South Dakota Site 5 Right, IRI Filter.

\begin{tabular}{|c|c|c|c|c|c|c|}
\hline Correlation & \multicolumn{6}{|c|}{ Correlated Device } \\
Reference & 01 & 05 & 08 & 10 & 11 & DS \\
\hline 01 & 81 & 83 & 85 & 30 & 73 & 82 \\
\hline 05 & 75 & $\mathbf{9 6}$ & 85 & 29 & 71 & 80 \\
\hline 08 & 84 & 85 & $\mathbf{9 6}$ & 27 & 81 & 86 \\
\hline 10 & 29 & 31 & 26 & 86 & 31 & 21 \\
\hline 11 & 79 & 78 & 87 & 33 & 83 & 75 \\
\hline DS & 75 & 87 & 85 & 21 & 68 & - \\
\hline
\end{tabular}

Table B-215. South Dakota Site 5 Right, Long Wavelengths.

\begin{tabular}{|c|c|c|c|c|c|c|}
\hline Correlation & \multicolumn{5}{|c|}{ Correlated Device } \\
Reference & 01 & 05 & 08 & 10 & 11 & DS \\
\hline 01 & 92 & 84 & 88 & 65 & 70 & 83 \\
\hline 05 & 82 & 99 & 93 & 64 & 77 & 82 \\
\hline 08 & 87 & 93 & 98 & 60 & 81 & 79 \\
\hline 10 & 65 & 64 & 60 & 78 & 54 & 67 \\
\hline 11 & 69 & 78 & 81 & 54 & 82 & 65 \\
\hline DS & 82 & 83 & 79 & 67 & 64 & - \\
\hline
\end{tabular}

Table B-216. South Dakota Site 5 Right, Medium Wavelengths.

\begin{tabular}{|c|c|c|c|c|c|c|}
\hline $\begin{array}{c}\text { Correlation } \\
\text { Reference }\end{array}$ & \multicolumn{6}{|c|}{ Correlated Device } \\
\hline 01 & 79 & 05 & 08 & 10 & 11 & DS \\
\hline 05 & 74 & 95 & 82 & 32 & 71 & 81 \\
\hline 08 & 83 & 85 & 94 & 29 & 70 & 80 \\
\hline 10 & 30 & 32 & 28 & 84 & 33 & 85 \\
\hline 11 & 79 & 78 & 86 & 36 & 83 & 75 \\
\hline DS & 73 & 88 & 83 & 22 & 67 & - \\
\hline
\end{tabular}

Table B-217. South Dakota Site 5 Right, Short Wavelengths.

\begin{tabular}{|c|c|c|c|c|c|c|}
\hline Correlation & \multicolumn{6}{|c|}{ Correlated Device } \\
Reference & 01 & 05 & 08 & 10 & 11 & DS \\
\hline 01 & 49 & 38 & 42 & 6 & 41 & 38 \\
\hline 05 & 37 & 54 & 64 & 5 & 43 & 43 \\
\hline 08 & 39 & 64 & 73 & 6 & 46 & 48 \\
\hline 10 & 10 & 8 & 10 & 35 & 12 & 11 \\
\hline 11 & 28 & 26 & 29 & 5 & 42 & 26 \\
\hline DS & 31 & 37 & 48 & 6 & 35 & - \\
\hline
\end{tabular}


Table B-218. South Dakota Site 6 Left, IRI Filter.

\begin{tabular}{|c|c|c|c|c|c|c|c|c|c|c|c|}
\hline $\begin{array}{c}\text { Correlation } \\
\text { Reference }\end{array}$ & \multicolumn{10}{|c|}{ Correlated Device } \\
\hline 01 & 82 & 67 & 66 & 68 & 73 & 70 & 74 & 58 & 68 & 67 & 74 \\
\hline 02 & 64 & 64 & 69 & 64 & 72 & 67 & 72 & 51 & 66 & 62 & 70 \\
\hline 03 & 63 & 64 & 82 & 71 & 82 & 73 & 81 & 55 & 66 & 65 & 81 \\
\hline 04 & 65 & 65 & 75 & 71 & 80 & 72 & 81 & 55 & 66 & 66 & 75 \\
\hline 05 & 70 & 69 & 85 & 78 & $\mathbf{9 5}$ & 80 & $\mathbf{9 5}$ & 61 & 71 & 70 & 86 \\
\hline 06 & 66 & 67 & 78 & 72 & 82 & 79 & 81 & 62 & 70 & 70 & 80 \\
\hline 08 & 71 & 70 & 83 & 79 & $\mathbf{9 5}$ & 80 & $\mathbf{9 5}$ & 62 & 71 & 70 & 84 \\
\hline 10 & 56 & 50 & 58 & 56 & 62 & 63 & 63 & 86 & 55 & 60 & 66 \\
\hline 11 & 64 & 65 & 70 & 68 & 74 & 74 & 75 & 56 & 77 & 64 & 72 \\
\hline 12 & 64 & 62 & 69 & 65 & 70 & 70 & 70 & 58 & 61 & 81 & 82 \\
\hline DS & 70 & 70 & 82 & 75 & 84 & 80 & 83 & 63 & 68 & 82 & - \\
\hline
\end{tabular}

Table B-219. South Dakota Site 6 Left, Long Wavelengths.

\begin{tabular}{|c|c|c|c|c|c|c|c|c|c|c|c|}
\hline $\begin{array}{c}\text { Correlation } \\
\text { Reference }\end{array}$ & \multicolumn{10}{|c|}{ Correlated Device } \\
\hline 01 & 98 & 74 & 71 & 77 & 85 & 77 & 86 & 73 & 72 & 59 & 79 \\
\hline 02 & 73 & 96 & 95 & 93 & 83 & 93 & 81 & 72 & 78 & 67 & 87 \\
\hline 03 & 71 & 95 & 96 & 92 & 83 & 92 & 82 & 68 & 78 & 62 & 83 \\
\hline 04 & 76 & 93 & 93 & 96 & 88 & 96 & 86 & 73 & 81 & 62 & 89 \\
\hline 05 & 85 & 83 & 83 & 88 & 99 & 87 & 98 & 69 & 84 & 51 & 80 \\
\hline 06 & 77 & 93 & 92 & 96 & 87 & 97 & 85 & 76 & 82 & 64 & 90 \\
\hline 08 & 86 & 81 & 82 & 86 & 98 & 85 & 99 & 68 & 82 & 50 & 79 \\
\hline 10 & 73 & 73 & 68 & 74 & 69 & 77 & 67 & 95 & 64 & 74 & 90 \\
\hline 11 & 72 & 79 & 78 & 82 & 83 & 83 & 81 & 63 & 93 & 53 & 75 \\
\hline 12 & 58 & 66 & 61 & 61 & 51 & 64 & 49 & 73 & 53 & 97 & 77 \\
\hline DS & 79 & 87 & 83 & 89 & 80 & 90 & 78 & 89 & 75 & 78 & - \\
\hline
\end{tabular}


Table B-220. South Dakota Site 6 Left, Medium Wavelengths.

\begin{tabular}{|c|c|c|c|c|c|c|c|c|c|c|c|}
\hline $\begin{array}{c}\text { Correlation } \\
\text { Reference }\end{array}$ & \multicolumn{10}{|c|}{ Correlated Device } \\
\hline 01 & 69 & 56 & 68 & 63 & 67 & 66 & 68 & 49 & 66 & 70 & 71 \\
\hline 02 & 59 & 50 & 58 & 49 & 54 & 51 & 55 & 37 & 58 & 53 & 54 \\
\hline 03 & 60 & 49 & 74 & 61 & 79 & 63 & 79 & 46 & 63 & 65 & 79 \\
\hline 04 & 59 & 50 & 68 & 59 & 68 & 58 & 68 & 43 & 60 & 61 & 63 \\
\hline 05 & 60 & 50 & 74 & 62 & 91 & 63 & 90 & 47 & 62 & 66 & 83 \\
\hline 06 & 59 & 51 & 68 & 58 & 69 & 66 & 69 & 48 & 65 & 63 & 65 \\
\hline 08 & 62 & 50 & 74 & 63 & 90 & 64 & 89 & 48 & 63 & 67 & 82 \\
\hline 10 & 47 & 35 & 50 & 43 & 51 & 50 & 51 & 78 & 49 & 45 & 49 \\
\hline 11 & 59 & 50 & 69 & 58 & 69 & 65 & 69 & 48 & 67 & 65 & 72 \\
\hline 12 & 62 & 53 & 70 & 61 & 71 & 63 & 71 & 43 & 63 & 71 & 73 \\
\hline DS & 63 & 54 & 73 & 62 & 83 & 65 & 82 & 46 & 64 & 73 & - \\
\hline
\end{tabular}

Table B-221. South Dakota Site 6 Left, Short Wavelengths.

\begin{tabular}{|c|c|c|c|c|c|c|c|c|c|c|c|}
\hline $\begin{array}{c}\text { Correlation } \\
\text { Reference }\end{array}$ & 01 & 02 & 03 & 04 & 05 & 06 & 08 & 10 & 11 & 12 & DS \\
\hline 01 & 27 & 9 & 28 & 10 & 20 & 12 & 21 & 6 & 25 & 15 & 27 \\
\hline 02 & 18 & 13 & 15 & 9 & 9 & 10 & 10 & 7 & 17 & 13 & 12 \\
\hline 03 & 16 & 6 & 32 & 9 & 18 & 8 & 22 & 4 & 19 & 10 & 30 \\
\hline 04 & 16 & 9 & 22 & 14 & 17 & 12 & 16 & 6 & 18 & 14 & 13 \\
\hline 05 & 16 & 7 & 27 & 13 & 42 & 10 & 43 & 4 & 18 & 13 & 31 \\
\hline 06 & 18 & 10 & 20 & 11 & 13 & 19 & 12 & 6 & 23 & 12 & 11 \\
\hline 08 & 18 & 8 & 26 & 12 & 43 & 10 & 46 & 4 & 19 & 13 & 28 \\
\hline 10 & 10 & 5 & 12 & 7 & 7 & 7 & 6 & 26 & 13 & 9 & 9 \\
\hline 11 & 16 & 7 & 23 & 8 & 13 & 10 & 15 & 4 & 25 & 10 & 26 \\
\hline 12 & 20 & 12 & 25 & 14 & 17 & 12 & 16 & 7 & 21 & 24 & 19 \\
\hline DS & 17 & 12 & 26 & 12 & 20 & 11 & 24 & 4 & 20 & 19 & - \\
\hline
\end{tabular}


Table B-222. South Dakota Site 6 Right, IRI Filter.

\begin{tabular}{|c|c|c|c|c|c|c|}
\hline $\begin{array}{c}\text { Correlation } \\
\text { Reference }\end{array}$ & \multicolumn{6}{|c|}{ Correlated Device } \\
\hline 01 & 01 & 05 & 08 & 10 & 11 & DS \\
\hline 05 & 81 & 73 & 75 & 53 & 60 & 72 \\
\hline 08 & 71 & $\mathbf{9 6}$ & $\mathbf{9 6}$ & 62 & 80 & 90 \\
\hline 10 & 52 & $\mathbf{9 6}$ & $\mathbf{9 7}$ & 61 & 80 & 89 \\
\hline 11 & 57 & 81 & 81 & 50 & 51 & 69 \\
\hline DS & 69 & 88 & 88 & 70 & 71 & - \\
\hline
\end{tabular}

Table B-223. South Dakota Site 6 Right, Long Wavelengths.

\begin{tabular}{|c|c|c|c|c|c|c|}
\hline Correlation & \multicolumn{5}{|c|}{ Correlated Device } \\
Reference & 01 & 05 & 08 & 10 & 11 & DS \\
\hline 01 & 98 & 82 & 85 & 64 & 63 & 79 \\
\hline 05 & 82 & 99 & 96 & 65 & 79 & 86 \\
\hline 08 & 85 & 96 & 99 & 62 & 76 & 83 \\
\hline 10 & 64 & 65 & 62 & 91 & 62 & 83 \\
\hline 11 & 63 & 79 & 76 & 62 & 91 & 75 \\
\hline DS & 79 & 86 & 83 & 84 & 76 & - \\
\hline
\end{tabular}

Table B-224. South Dakota Site 6 Right, Medium Wavelengths.

\begin{tabular}{|c|c|c|c|c|c|c|}
\hline $\begin{array}{c}\text { Correlation } \\
\text { Reference }\end{array}$ & \multicolumn{5}{|c|}{ Correlated Device } \\
\hline 01 & 63 & 05 & 08 & 10 & 11 & DS \\
\hline 05 & 66 & 92 & 91 & 58 & 71 & 86 \\
\hline 08 & 65 & 91 & 93 & 58 & 70 & 89 \\
\hline 10 & 42 & 56 & 57 & 83 & 49 & 58 \\
\hline 11 & 57 & 78 & 77 & 52 & 71 & 70 \\
\hline DS & 60 & 82 & 85 & 55 & 63 & - \\
\hline
\end{tabular}

Table B-225. South Dakota Site 6 Right, Short Wavelengths.

\begin{tabular}{|c|c|c|c|c|c|c|}
\hline $\begin{array}{c}\text { Correlation } \\
\text { Reference }\end{array}$ & \multicolumn{6}{|c|}{ Correlated Device } \\
\hline 01 & 27 & 32 & 33 & 6 & 23 & 17 \\
\hline 05 & 21 & 54 & 54 & 4 & 26 & 22 \\
\hline 08 & 21 & 54 & 54 & 4 & 24 & 21 \\
\hline 10 & 6 & 8 & 8 & 30 & 9 & 7 \\
\hline 11 & 13 & 22 & 23 & 5 & 24 & 13 \\
\hline DS & 15 & 34 & 38 & 6 & 20 & - \\
\hline
\end{tabular}


Table B-226. South Dakota Site 7 Left, IRI Filter.

\begin{tabular}{|c|c|c|c|c|c|c|c|c|c|c|c|}
\hline $\begin{array}{c}\text { Correlation } \\
\text { Reference }\end{array}$ & \multicolumn{10}{|c|}{ Correlated Device } \\
\hline 01 & 80 & 71 & 66 & 60 & 75 & 65 & 74 & 27 & 65 & 63 & 85 \\
\hline 02 & 73 & 72 & 68 & 66 & 70 & 68 & 75 & 26 & 69 & 69 & 79 \\
\hline 03 & 73 & 73 & 77 & 74 & 70 & 75 & 79 & 25 & 76 & 78 & 79 \\
\hline 04 & 66 & 66 & 74 & 75 & 63 & 74 & 75 & 25 & 76 & 79 & 68 \\
\hline 05 & 71 & 68 & 64 & 59 & 76 & 62 & 78 & 23 & 62 & 63 & 78 \\
\hline 06 & 71 & 68 & 75 & 74 & 65 & 84 & 78 & 32 & 81 & 79 & 68 \\
\hline 08 & 79 & 75 & 76 & 71 & 78 & 75 & $\mathbf{9 6}$ & 26 & 75 & 75 & 80 \\
\hline 10 & 30 & 28 & 25 & 24 & 25 & 30 & 28 & 82 & 32 & 25 & 25 \\
\hline 11 & 71 & 73 & 75 & 72 & 67 & 79 & 81 & 32 & 82 & 77 & 74 \\
\hline 12 & 69 & 69 & 77 & 79 & 66 & 79 & 79 & 26 & 81 & 83 & 72 \\
\hline DS & 82 & 79 & 71 & 68 & 81 & 68 & 80 & 23 & 68 & 72 & - \\
\hline
\end{tabular}

Table B-227. South Dakota Site 7 Left, Long Wavelengths.

\begin{tabular}{|c|c|c|c|c|c|c|c|c|c|c|c|}
\hline $\begin{array}{c}\text { Correlation } \\
\text { Reference }\end{array}$ & \multicolumn{10}{|c|}{ Correlated Device } \\
\hline 01 & 93 & 77 & 78 & 82 & 84 & 84 & 87 & 75 & 72 & 84 & 91 \\
\hline 02 & 76 & 97 & 91 & 87 & 86 & 87 & 82 & 60 & 69 & 87 & 81 \\
\hline 03 & 77 & 93 & 95 & 91 & 90 & 91 & 86 & 61 & 72 & 91 & 82 \\
\hline 04 & 81 & 87 & 92 & 93 & 91 & 93 & 88 & 63 & 73 & 94 & 87 \\
\hline 05 & 83 & 87 & 90 & 91 & 95 & 92 & 92 & 64 & 74 & 92 & 86 \\
\hline 06 & 83 & 87 & 92 & 93 & 93 & 95 & 89 & 67 & 75 & 95 & 88 \\
\hline 08 & 87 & 82 & 87 & 89 & 92 & 90 & 97 & 68 & 79 & 91 & 84 \\
\hline 10 & 76 & 60 & 62 & 63 & 64 & 67 & 68 & 88 & 62 & 64 & 73 \\
\hline 11 & 74 & 70 & 74 & 74 & 75 & 76 & 80 & 63 & 87 & 78 & 70 \\
\hline 12 & 83 & 87 & 93 & 94 & 93 & 95 & 90 & 64 & 76 & 97 & 89 \\
\hline DS & 90 & 81 & 83 & 87 & 86 & 88 & 84 & 73 & 68 & 89 & - \\
\hline
\end{tabular}


Table B-228. South Dakota Site 7 Left, Medium Wavelengths.

\begin{tabular}{|c|c|c|c|c|c|c|c|c|c|c|c|}
\hline $\begin{array}{c}\text { Correlation } \\
\text { Reference }\end{array}$ & \multicolumn{10}{|c|}{ Correlated Device } \\
\hline 01 & 76 & 64 & 62 & 53 & 72 & 59 & 72 & 24 & 63 & 58 & 80 \\
\hline 02 & 72 & 69 & 67 & 61 & 67 & 65 & 74 & 24 & 68 & 66 & 74 \\
\hline 03 & 71 & 71 & 76 & 68 & 67 & 72 & 79 & 26 & 76 & 74 & 77 \\
\hline 04 & 61 & 61 & 73 & 70 & 57 & 69 & 69 & 24 & 75 & 75 & 62 \\
\hline 05 & 68 & 62 & 60 & 53 & 73 & 57 & 77 & 20 & 60 & 58 & 75 \\
\hline 06 & 67 & 65 & 74 & 69 & 61 & 80 & 74 & 34 & 80 & 75 & 64 \\
\hline 08 & 77 & 73 & 72 & 64 & 77 & 69 & 95 & 23 & 72 & 70 & 81 \\
\hline 10 & 28 & 26 & 26 & 22 & 22 & 31 & 26 & 79 & 32 & 24 & 25 \\
\hline 11 & 70 & 73 & 75 & 66 & 65 & 75 & 80 & 32 & 80 & 74 & 75 \\
\hline 12 & 65 & 66 & 77 & 75 & 62 & 75 & 75 & 26 & 80 & 80 & 69 \\
\hline DS & 79 & 74 & 68 & 62 & 79 & 64 & 79 & 22 & 68 & 69 & - \\
\hline
\end{tabular}

Table B-229. South Dakota Site 7 Left, Short Wavelengths.

\begin{tabular}{|c|c|c|c|c|c|c|c|c|c|c|c|}
\hline $\begin{array}{c}\text { Correlation } \\
\text { Reference }\end{array}$ & 01 & 02 & 03 & 04 & 05 & 06 & 08 & 10 & 11 & 12 & DS \\
\hline 01 & 43 & 21 & 33 & 14 & 31 & 22 & 34 & 4 & 35 & 24 & 42 \\
\hline 02 & 34 & 32 & 37 & 23 & 31 & 24 & 37 & 5 & 37 & 31 & 35 \\
\hline 03 & 35 & 24 & 47 & 17 & 32 & 20 & 36 & 3 & 39 & 26 & 47 \\
\hline 04 & 28 & 23 & 34 & 20 & 24 & 18 & 29 & 5 & 30 & 25 & 24 \\
\hline 05 & 30 & 27 & 30 & 19 & 46 & 22 & 58 & 3 & 32 & 29 & 51 \\
\hline 06 & 35 & 24 & 32 & 18 & 25 & 34 & 30 & 4 & 38 & 23 & 22 \\
\hline 08 & 34 & 33 & 36 & 23 & 58 & 27 & 75 & 3 & 41 & 35 & 59 \\
\hline 10 & 10 & 8 & 8 & 7 & 6 & 7 & 7 & 36 & 9 & 8 & 4 \\
\hline 11 & 29 & 19 & 30 & 13 & 25 & 20 & 28 & 3 & 37 & 21 & 34 \\
\hline 12 & 35 & 31 & 38 & 25 & 34 & 23 & 39 & 4 & 39 & 37 & 40 \\
\hline DS & 32 & 35 & 37 & 24 & 37 & 22 & 42 & 3 & 34 & 40 & - \\
\hline
\end{tabular}


Table B-230. South Dakota Site 7 Right, IRI Filter.

\begin{tabular}{|c|c|c|c|c|c|c|}
\hline Correlation & \multicolumn{6}{|c|}{ Correlated Device } \\
\hline Reference & 01 & 05 & 08 & 10 & 11 & DS \\
\hline 01 & 85 & 88 & 89 & 35 & 76 & 87 \\
\hline 05 & 81 & 96 & 93 & 35 & 76 & 90 \\
\hline 08 & 85 & 93 & 97 & 32 & 81 & 91 \\
\hline 10 & 32 & 32 & 30 & 89 & 30 & 31 \\
\hline 11 & 82 & 83 & 87 & 32 & 85 & 82 \\
\hline DS & 82 & 95 & 91 & 33 & 76 & - \\
\hline
\end{tabular}

Table B-231. South Dakota Site 7 Right, Long Wavelengths.

\begin{tabular}{|c|c|c|c|c|c|c|}
\hline Correlation & \multicolumn{5}{|c|}{ Correlated Device } \\
Reference & 01 & 05 & 08 & 10 & 11 & DS \\
\hline 01 & 95 & 86 & 88 & 69 & 71 & 88 \\
\hline 05 & 85 & 99 & 97 & 57 & 82 & 86 \\
\hline 08 & 87 & 97 & 98 & 56 & 82 & 83 \\
\hline 10 & 69 & 57 & 56 & 81 & 51 & 72 \\
\hline 11 & 70 & 82 & 82 & 51 & 86 & 71 \\
\hline DS & 87 & 86 & 83 & 72 & 70 & - \\
\hline
\end{tabular}

Table B-232. South Dakota Site 7 Right, Medium Wavelengths.

\begin{tabular}{|c|c|c|c|c|c|c|}
\hline Correlation & \multicolumn{6}{|c|}{ Correlated Device } \\
Reference & 01 & 05 & 08 & 10 & 11 & DS \\
\hline 01 & 83 & 88 & 87 & 38 & 73 & 86 \\
\hline 05 & 80 & 95 & 93 & 36 & 74 & 89 \\
\hline 08 & 83 & 93 & 96 & 35 & 78 & 89 \\
\hline 10 & 33 & 34 & 33 & 88 & 34 & 33 \\
\hline 11 & 81 & 82 & 85 & 37 & 84 & 81 \\
\hline DS & 80 & 94 & 91 & 36 & 73 & - \\
\hline
\end{tabular}

Table B-233. South Dakota Site 7 Right, Short Wavelengths.

\begin{tabular}{|c|c|c|c|c|c|c|}
\hline $\begin{array}{c}\text { Correlation } \\
\text { Reference }\end{array}$ & \multicolumn{6}{|c|}{ Correlated Device } \\
\hline 01 & 52 & 36 & 46 & 7 & 41 & 46 \\
\hline 05 & 41 & 60 & 62 & 6 & 44 & 56 \\
\hline 08 & 43 & 62 & 77 & 6 & 47 & 45 \\
\hline 10 & 12 & 10 & 12 & 50 & 17 & 16 \\
\hline 11 & 30 & 25 & 31 & 6 & 43 & 33 \\
\hline DS & 39 & 52 & 62 & 8 & 44 & - \\
\hline
\end{tabular}


Table B-234. South Dakota Site 8 Left, IRI Filter.

\begin{tabular}{|c|c|c|c|c|c|c|c|c|c|c|}
\hline $\begin{array}{c}\text { Correlation } \\
\text { Reference }\end{array}$ & \multicolumn{9}{|c|}{ Correlated Device } \\
\hline 01 & 85 & 57 & 71 & 73 & 77 & 73 & 74 & 70 & 75 & 77 \\
\hline 02 & 62 & 49 & 54 & 50 & 54 & 51 & 52 & 59 & 53 & 52 \\
\hline 03 & 67 & 48 & 79 & 74 & 83 & 72 & 82 & 68 & 75 & 82 \\
\hline 04 & 69 & 50 & 78 & 77 & 85 & 72 & 83 & 68 & 77 & 77 \\
\hline 05 & 73 & 51 & 84 & 82 & $\mathbf{9 6}$ & 77 & $\mathbf{9 5}$ & 72 & 81 & 86 \\
\hline 06 & 69 & 51 & 75 & 72 & 79 & 77 & 77 & 69 & 74 & 77 \\
\hline 08 & 70 & 50 & 82 & 79 & $\mathbf{9 5}$ & 74 & $\mathbf{9 7}$ & 70 & 78 & 86 \\
\hline 11 & 67 & 52 & 72 & 71 & 76 & 72 & 74 & 80 & 75 & 72 \\
\hline 12 & 71 & 52 & 78 & 77 & 84 & 74 & 81 & 72 & 80 & 81 \\
\hline DS & 73 & 52 & 81 & 77 & 85 & 77 & 84 & 68 & 81 & - \\
\hline
\end{tabular}

Table B-235. South Dakota Site 8 Left, Long Wavelengths.

\begin{tabular}{|c|c|c|c|c|c|c|c|c|c|c|}
\hline Correlation & \multicolumn{9}{|c|}{ Correlated Device } \\
Reference & 01 & 02 & 03 & 04 & 05 & 06 & 08 & 11 & 12 & DS \\
\hline 01 & 97 & 75 & 73 & 76 & 85 & 78 & 85 & 78 & 77 & 81 \\
\hline 02 & 75 & 93 & 93 & 91 & 84 & 93 & 81 & 68 & 93 & 87 \\
\hline 03 & 72 & 92 & 94 & 92 & 83 & 91 & 80 & 67 & 93 & 85 \\
\hline 04 & 75 & 91 & 92 & 92 & 84 & 92 & 82 & 65 & 93 & 86 \\
\hline 05 & 85 & 84 & 83 & 84 & 99 & 86 & 98 & 76 & 86 & 78 \\
\hline 06 & 78 & 93 & 92 & 92 & 86 & 95 & 83 & 69 & 94 & 89 \\
\hline 08 & 85 & 81 & 80 & 82 & 98 & 83 & 99 & 77 & 83 & 76 \\
\hline 11 & 79 & 68 & 67 & 66 & 77 & 70 & 77 & 88 & 69 & 66 \\
\hline 12 & 77 & 93 & 93 & 93 & 86 & 94 & 83 & 69 & 95 & 89 \\
\hline DS & 81 & 87 & 85 & 86 & 78 & 89 & 76 & 65 & 89 & - \\
\hline
\end{tabular}


Table B-236. South Dakota Site 8 Left, Medium Wavelengths.

\begin{tabular}{|c|c|c|c|c|c|c|c|c|c|c|}
\hline $\begin{array}{c}\text { Correlation } \\
\text { Reference }\end{array}$ & \multicolumn{9}{|c|}{ Correlated Device } \\
\hline 01 & 73 & 35 & 71 & 64 & 77 & 64 & 73 & 66 & 67 & 73 \\
\hline 02 & 43 & 32 & 41 & 34 & 38 & 34 & 36 & 43 & 36 & 34 \\
\hline 03 & 65 & 33 & 70 & 63 & 77 & 59 & 76 & 64 & 64 & 77 \\
\hline 04 & 64 & 34 & 69 & 66 & 75 & 58 & 71 & 64 & 66 & 63 \\
\hline 05 & 67 & 33 & 73 & 67 & 92 & 61 & 90 & 64 & 67 & 79 \\
\hline 06 & 63 & 34 & 64 & 58 & 68 & 66 & 64 & 64 & 61 & 59 \\
\hline 08 & 63 & 32 & 69 & 64 & 90 & 58 & 94 & 60 & 64 & 80 \\
\hline 11 & 63 & 35 & 67 & 60 & 71 & 62 & 68 & 68 & 64 & 72 \\
\hline 12 & 67 & 36 & 70 & 66 & 74 & 61 & 71 & 68 & 70 & 67 \\
\hline DS & 64 & 34 & 67 & 63 & 75 & 59 & 76 & 64 & 67 & - \\
\hline
\end{tabular}

Table B-237. South Dakota Site 8 Left, Short Wavelengths.

\begin{tabular}{|c|c|c|c|c|c|c|c|c|c|c|}
\hline $\begin{array}{c}\text { Correlation } \\
\text { Reference }\end{array}$ & 01 & 02 & 03 & 04 & 05 & 06 & 08 & 11 & 12 & DS \\
\hline 01 & 42 & 6 & 35 & 19 & 38 & 18 & 40 & 31 & 21 & 38 \\
\hline 02 & 13 & 9 & 12 & 8 & 11 & 6 & 11 & 12 & 9 & 6 \\
\hline 03 & 27 & 5 & 40 & 17 & 32 & 13 & 35 & 28 & 18 & 36 \\
\hline 04 & 28 & 8 & 33 & 28 & 38 & 15 & 40 & 28 & 29 & 25 \\
\hline 05 & 36 & 7 & 42 & 27 & 68 & 21 & 71 & 34 & 27 & 51 \\
\hline 06 & 25 & 6 & 24 & 15 & 26 & 24 & 28 & 22 & 16 & 12 \\
\hline 08 & 36 & 7 & 41 & 28 & 71 & 21 & 79 & 34 & 28 & 52 \\
\hline 11 & 21 & 5 & 25 & 13 & 23 & 10 & 25 & 26 & 15 & 31 \\
\hline 12 & 30 & 9 & 34 & 29 & 38 & 16 & 41 & 30 & 32 & 26 \\
\hline DS & 24 & 6 & 30 & 25 & 32 & 12 & 35 & 30 & 26 & - \\
\hline
\end{tabular}


Table B-238. South Dakota Site 8 Right, IRI Filter.

\begin{tabular}{|c|c|c|c|c|c|}
\hline Correlation & \multicolumn{5}{|c|}{ Correlated Device } \\
Reference & 01 & 05 & 08 & 11 & DS \\
\hline 01 & 82 & 75 & 81 & 64 & 79 \\
\hline 05 & 71 & $\mathbf{9 5}$ & 91 & 77 & 88 \\
\hline 08 & 76 & 91 & $\mathbf{9 6}$ & 78 & 91 \\
\hline 11 & 60 & 75 & 75 & 76 & 75 \\
\hline DS & 74 & 88 & 90 & 77 & - \\
\hline
\end{tabular}

Table B-239. South Dakota Site 8 Right, Long Wavelengths.

\begin{tabular}{|c|c|c|c|c|c|}
\hline Correlation & \multicolumn{5}{|c|}{ Correlated Device } \\
Reference & 01 & 05 & 08 & 11 & DS \\
\hline 01 & 98 & 82 & 85 & 52 & 84 \\
\hline 05 & 81 & 99 & 96 & 69 & 86 \\
\hline 08 & 85 & 96 & 99 & 66 & 83 \\
\hline 11 & 51 & 69 & 66 & 81 & 57 \\
\hline DS & 83 & 85 & 83 & 57 & - \\
\hline
\end{tabular}

Table B-240. South Dakota Site 8 Right, Medium Wavelengths.

\begin{tabular}{|c|c|c|c|c|c|}
\hline Correlation & \multicolumn{5}{|c|}{ Correlated Device } \\
Reference & 01 & 05 & 08 & 11 & DS \\
\hline 01 & 72 & 76 & 82 & 70 & 78 \\
\hline 05 & 69 & 93 & 87 & 75 & 85 \\
\hline 08 & 72 & 87 & 93 & 76 & 88 \\
\hline 11 & 62 & 70 & 78 & 72 & 74 \\
\hline DS & 69 & 79 & 88 & 74 & - \\
\hline
\end{tabular}

Table B-241. South Dakota Site 8 Right, Short Wavelengths.

\begin{tabular}{|c|c|c|c|c|c|}
\hline Correlation & \multicolumn{5}{|c|}{ Correlated Device } \\
Reference & 01 & 05 & 08 & 11 & DS \\
\hline 01 & 39 & 33 & 41 & 31 & 35 \\
\hline 05 & 39 & 76 & 60 & 39 & 54 \\
\hline 08 & 33 & 60 & 70 & 34 & 44 \\
\hline 11 & 21 & 22 & 26 & 29 & 24 \\
\hline DS & 30 & 39 & 47 & 33 & - \\
\hline
\end{tabular}





\section{Appendix C: Interpretation of 1993 RPUG Cross Correlation Results}

This appendix interprets the cross correlation results from the 1993 RPUG study. These analyses establish a relationship between cross correlation level and scatter, RMS error, and individual error in summary IRI measurement. First, overall results for repeatability in IRI measurement (listed in Appendix A) are compared to the appropriate cross correlation values (listed in Appendix B). Second, RMS difference in IRI measurement compared to the Dipstick (listed in Appendix A) is compared to cross correlation of profile with Dipstick measurements (listed in Appendix B). Third, all possible pairs of measurement from the 1993 RPUG study are examined. In this case, every possible IRI comparison (all measurement on a given site compared to all others) are compared to every possible cross correlation value between the corresponding pairs of profiles. This covers 378,758 paired measurements. The analyses are repeated with profiles from devices with ultrasonic sensors eliminated, since they are no longer in use.

\section{REPEATABILITY}

In Appendix A, a composite level of repeatability was calculated for each of 33 inertial profilers from the 1993 RPUG study. Each individual measured IRI value was compared to the average by a given device on a given site and wheeltrack. Often, 10 measurements were made on both wheeltracks of 8 sites. Thus, the 10 individual IRI values for a given wheeltrack were compared to their average. This was repeated for each site (and wheeltrack), and all of the values (usually 160) of percent difference from the average were assembled into a distribution for that device. (See figure A-1.) The standard deviation exhibited by each device is listed in tables $\mathrm{C}-1$ and $\mathrm{C}-2$. These values quantify the scatter in IRI measurement, and represent an overall assessment of repeatability.

The tables also list the average cross correlation values for each device when all of its measurements of a given wheeltrack are compared to each other. In this case, the IRI filter was used in the procedure. These values are the average of all cross correlation values for all possible combinations of repeat measurements (i.e., repeat 1 to repeat 2, repeat 1 to repeat 3 , etc.). When 10 repeat measurements were made, the corresponding value in the table represents 45 comparisons. Table $\mathrm{C}-1$ lists the results for the left wheeltrack and table $\mathrm{C}-2$ lists the results for the right. (Not every device measured both wheeltracks, and some devices did not measure some of the sites at all.)

Overall, a vague relationship exists between the ratings of "scatter" and the cross correlation values. The lowest level of scatter ( 1.63 percent) in table $\mathrm{C}-1$ is exhibited by device S08, and is accompanied by cross correlation values for all 8 sites greater than 0.90 . The highest level of scatter (10.60 percent) is exhibited by device S03, which shows some of the lowest cross correlation values. An important observation to be made about the cross correlation values is that they depend on the test site as well as the device. Several of the profilers have high cross correlation levels on some sites and low levels on others. This is 
because some sites are more problematic to some profilers than others. Since the listed standard deviation values often pertain to both wheeltracks of all sites, a direct comparison to cross correlation level is not possible.

Table C-1. Repeatability of profilers in measurement of IRI, left side.

\begin{tabular}{|c|c|c|c|c|c|c|c|c|c|c|c|}
\hline \multirow[t]{2}{*}{ Region } & \multirow[t]{2}{*}{ Device } & \multirow{2}{*}{$\begin{array}{c}\text { Sensor } \\
\text { Type }\end{array}$} & \multirow{2}{*}{$\begin{array}{c}\text { Std. Dev. } \\
(\%)\end{array}$} & \multicolumn{8}{|c|}{ Average Correlation at Each Site } \\
\hline & & & & 1 & 2 & 3 & 4 & 5 & 6 & 7 & 8 \\
\hline \multirow[t]{5}{*}{ M } & M01 & $\mathrm{U}$ & 4.77 & .78 & .71 & .52 & .53 & .87 & .81 & .80 & .57 \\
\hline & M02 & $\mathrm{U}$ & 4.74 & .81 & .49 & .44 & .60 & .82 & .74 & .82 & .55 \\
\hline & M03 & $\mathrm{L}$ & 3.59 & .79 & .71 & .78 & .74 & .94 & .86 & - & .87 \\
\hline & M05 & $\mathrm{U}$ & 7.80 & .61 & .62 & .47 & .57 & .87 & .46 & .65 & .50 \\
\hline & M06 & $\mathrm{O}$ & 1.92 & .88 & .92 & .82 & .90 & .97 & .94 & .98 & .93 \\
\hline \multirow[t]{6}{*}{$\mathrm{N}$} & N03 & $\mathrm{U}$ & 6.65 & .68 & .37 & .66 & .60 & .45 & .28 & - & - \\
\hline & N04 & $\mathrm{U}$ & 6.63 & .51 & .21 & .65 & .80 & .56 & .48 & - & - \\
\hline & N06 & $\mathrm{U}$ & 5.64 & .78 & .48 & .36 & .60 & .61 & .41 & - & - \\
\hline & N07 & $\mathrm{L}$ & 4.15 & .92 & .91 & .77 & .87 & .81 & .82 & - & - \\
\hline & N08 & $\mathrm{O}$ & 3.59 & .94 & .89 & .81 & .86 & .93 & .87 & - & - \\
\hline & N09 & $\mathrm{O}$ & 3.49 & .91 & .80 & .85 & .84 & .89 & .80 & - & - \\
\hline \multirow[t]{12}{*}{$\mathrm{P}$} & P01 & $\mathrm{L}$ & 2.72 & .96 & .92 & .91 & .96 & .95 & .94 & .94 & .93 \\
\hline & P02 & $\mathrm{U}$ & 3.76 & .90 & .59 & .48 & .61 & .83 & .86 & .84 & .73 \\
\hline & P03 & $\mathrm{U}$ & 3.87 & .89 & .49 & .46 & .63 & .82 & .89 & .89 & .83 \\
\hline & P04 & $\mathrm{L}$ & 3.80 & .96 & .71 & .87 & .86 & .91 & .95 & .91 & .81 \\
\hline & P05 & $\mathrm{O}$ & 1.86 & .92 & .87 & .96 & .97 & .95 & .96 & .95 & .96 \\
\hline & P06 & $\mathrm{U}$ & 7.46 & .90 & .49 & .25 & .52 & .86 & .75 & .60 & .74 \\
\hline & P07 & $\mathrm{O}$ & 2.57 & .93 & .82 & .91 & .93 & .97 & .96 & .87 & .60 \\
\hline & P08 & $\mathrm{L}$ & 2.37 & .88 & .73 & .92 & .93 & .92 & .95 & .92 & .88 \\
\hline & P73 & $\mathrm{U}$ & 3.84 & .92 & .61 & .47 & .55 & .86 & .86 & .82 & .77 \\
\hline & P74 & $\mathrm{U}$ & 3.00 & .92 & .79 & .52 & .67 & .87 & .89 & .84 & .77 \\
\hline & P75 & $\mathrm{U}$ & 3.20 & .90 & .69 & .49 & .63 & .85 & .85 & .81 & .76 \\
\hline & P76 & $\mathrm{U}$ & 2.28 & .91 & .81 & .51 & .69 & .87 & .88 & .80 & .73 \\
\hline \multirow[t]{10}{*}{$S$} & S01 & $\mathrm{U}$ & 3.57 & .81 & .30 & .42 & .30 & .76 & .82 & .80 & .85 \\
\hline & S02 & $\mathrm{U}$ & 7.62 & .83 & .49 & .64 & .36 & .69 & .64 & .72 & .49 \\
\hline & S03 & $\mathrm{U}$ & 10.60 & .70 & .16 & .29 & .20 & .73 & .82 & .77 & .79 \\
\hline & S04 & $\mathrm{U}$ & 5.65 & .67 & .25 & .29 & .23 & .58 & .71 & .75 & .77 \\
\hline & S05 & $\mathrm{O}$ & 5.47 & .94 & .91 & .91 & .89 & .92 & .95 & .76 & .96 \\
\hline & S06 & $\mathrm{U}$ & 4.78 & .73 & .23 & .28 & .20 & .83 & .79 & .84 & .77 \\
\hline & S08 & $\mathrm{O}$ & 1.63 & .95 & .93 & .95 & .92 & .95 & .95 & .96 & .97 \\
\hline & $\mathrm{S} 10$ & $\mathrm{~L}$ & 2.49 & .90 & .71 & .73 & .62 & .77 & .86 & .82 & - \\
\hline & S11 & $\mathrm{U}$ & 5.09 & .70 & .21 & .32 & .29 & .84 & .77 & .82 & .80 \\
\hline & $\mathrm{S} 12$ & $\mathrm{U}$ & 7.46 & .71 & .19 & .28 & .23 & .84 & .81 & .83 & .80 \\
\hline \multicolumn{2}{|c|}{$\begin{array}{l}\text { M - Mississippi } \\
\text { O - Optical }\end{array}$} & \multicolumn{2}{|c|}{$\begin{array}{l}\mathrm{N} \text { - Nevada } \\
\mathrm{U} \text { - Ultrasonic }\end{array}$} & & $\mathrm{Pe}$ & lva & & \multicolumn{4}{|c|}{ S - South Dakota } \\
\hline
\end{tabular}


Table C-2. Repeatability of profilers in measurement of IRI, right side.

\begin{tabular}{|c|c|c|c|c|c|c|c|c|c|c|c|}
\hline \multirow[t]{2}{*}{ Region } & \multirow[t]{2}{*}{ Device } & \multirow{2}{*}{$\begin{array}{c}\text { Sensor } \\
\text { Type }\end{array}$} & \multirow{2}{*}{$\begin{array}{c}\text { Std. Dev. } \\
(\%)\end{array}$} & \multicolumn{8}{|c|}{ Average Correlation at Each Site } \\
\hline & & & & 1 & 2 & 3 & 4 & 5 & 6 & 7 & 8 \\
\hline \multirow[t]{4}{*}{ M } & M02 & $\mathrm{U}$ & 4.74 & .80 & .55 & .55 & .66 & .84 & .77 & .79 & .57 \\
\hline & M03 & $\mathrm{L}$ & 3.59 & .90 & .90 & .76 & .84 & 97 & .92 & - & .93 \\
\hline & M05 & $\mathrm{U}$ & 7.80 & .75 & .68 & .39 & .47 & .89 & .60 & .59 & .57 \\
\hline & M06 & $\mathrm{O}$ & 1.92 & .95 & .94 & .89 & .95 & .97 & .98 & .98 & .97 \\
\hline \multirow[t]{6}{*}{$\mathrm{N}$} & N03 & U & 6.65 & .77 & .49 & .55 & .59 & .53 & .43 & - & - \\
\hline & N04 & $\mathrm{U}$ & 6.63 & .56 & .30 & .40 & .77 & .64 & .63 & - & - \\
\hline & N06 & $\mathrm{U}$ & 5.64 & .81 & .45 & .34 & .62 & .69 & .56 & - & - \\
\hline & N07 & $\mathrm{L}$ & 4.15 & .93 & .93 & .90 & .88 & .83 & .86 & - & - \\
\hline & N08 & $\mathrm{O}$ & 3.59 & .92 & .91 & .91 & .84 & .93 & .87 & - & - \\
\hline & N09 & $\mathrm{O}$ & 3.49 & .90 & .95 & .94 & .84 & .91 & .89 & - & - \\
\hline \multirow[t]{12}{*}{$P$} & P01 & $\mathrm{L}$ & 2.72 & .94 & .88 & .88 & .97 & .97 & .95 & .91 & .88 \\
\hline & $\mathrm{P} 02$ & $\mathrm{U}$ & 3.76 & .88 & .67 & .53 & .73 & .86 & .88 & .85 & .70 \\
\hline & $\mathrm{P} 03$ & $\mathrm{U}$ & 3.87 & .89 & .45 & .58 & .62 & .79 & .85 & .86 & .71 \\
\hline & $\mathrm{P} 04$ & $\mathrm{~L}$ & 3.80 & .95 & .60 & .84 & .83 & .88 & .79 & .91 & .78 \\
\hline & P05 & $\mathrm{O}$ & 1.86 & .95 & .86 & .94 & .96 & .96 & .96 & .96 & .93 \\
\hline & P06 & $\mathrm{U}$ & 7.46 & 89 & .49 & .48 & .79 & .88 & .89 & .85 & .79 \\
\hline & P07 & $\mathrm{O}$ & 2.57 & .96 & .83 & .92 & .94 & .98 & .98 & .94 & .94 \\
\hline & P08 & $\mathrm{L}$ & 2.37 & .92 & .88 & .91 & .95 & .95 & .96 & .95 & .88 \\
\hline & P73 & $\mathrm{U}$ & 3.84 & .92 & .62 & .53 & .62 & .89 & .88 & .83 & .74 \\
\hline & P74 & $\mathrm{U}$ & 3.00 & .91 & .75 & .59 & .66 & .82 & .90 & .84 & .70 \\
\hline & P75 & $\mathrm{U}$ & 3.20 & .90 & .70 & .52 & .79 & .88 & .87 & .81 & .71 \\
\hline & P76 & $\mathrm{U}$ & 2.28 & .92 & .81 & .48 & .83 & .89 & .89 & .86 & .71 \\
\hline \multirow[t]{5}{*}{$\bar{S}$} & S01 & $\mathrm{U}$ & 3.57 & .83 & .47 & .45 & .36 & .81 & .81 & .85 & .82 \\
\hline & S05 & $\mathrm{O}$ & 5.47 & .95 & .94 & .92 & .91 & .96 & .96 & .96 & .95 \\
\hline & S08 & $\mathrm{O}$ & 1.63 & .95 & .93 & .96 & .91 & .96 & .97 & .97 & .96 \\
\hline & S10 & $\mathrm{L}$ & 2.49 & .88 & .84 & .80 & .61 & .86 & .90 & .89 & - \\
\hline & S11 & $\mathrm{U}$ & 5.09 & .70 & .41 & .33 & .30 & .83 & .80 & .85 & .76 \\
\hline \multicolumn{2}{|c|}{$\begin{array}{l}\text { M - Mississippi } \\
\text { O - Optical }\end{array}$} & $\mathrm{U}-\mathrm{Ul}$ & & & Las & 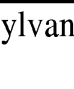 & & & & $\overline{\mathrm{Da}}$ & \\
\hline
\end{tabular}

To remedy this, a standard deviation value was calculated to correspond to each value for cross correlation in tables $\mathrm{C}-1$ and $\mathrm{C}-2$. Thus, new values of standard deviation were calculated that only include the error in measurement of IRI on one wheeltrack of one site by one device. Usually, this is the standard deviation of 10 values, representing 10 repeat measurements. When this is done, all 452 cross correlation values in the tables can be paired to a level of scatter in IRI for the appropriate combination of device and wheeltrack.

Figure $\mathrm{C}-1$ compares these values. When the cross correlation level is near 1 , the scatter in IRI (standard deviation) is near zero. This is because a cross correlation level of 1 indicates total agreement in the components of profile that affect the IRI. As the cross correlation level decreases, the largest observed scatter in IRI measurement increases. This is because the profiles themselves do not agree as well. In addition, when cross correlation decreases too far the potential for agreement in IRI caused by compensating error increases, so the range of RMS error values spans a range from zero to some large value. 


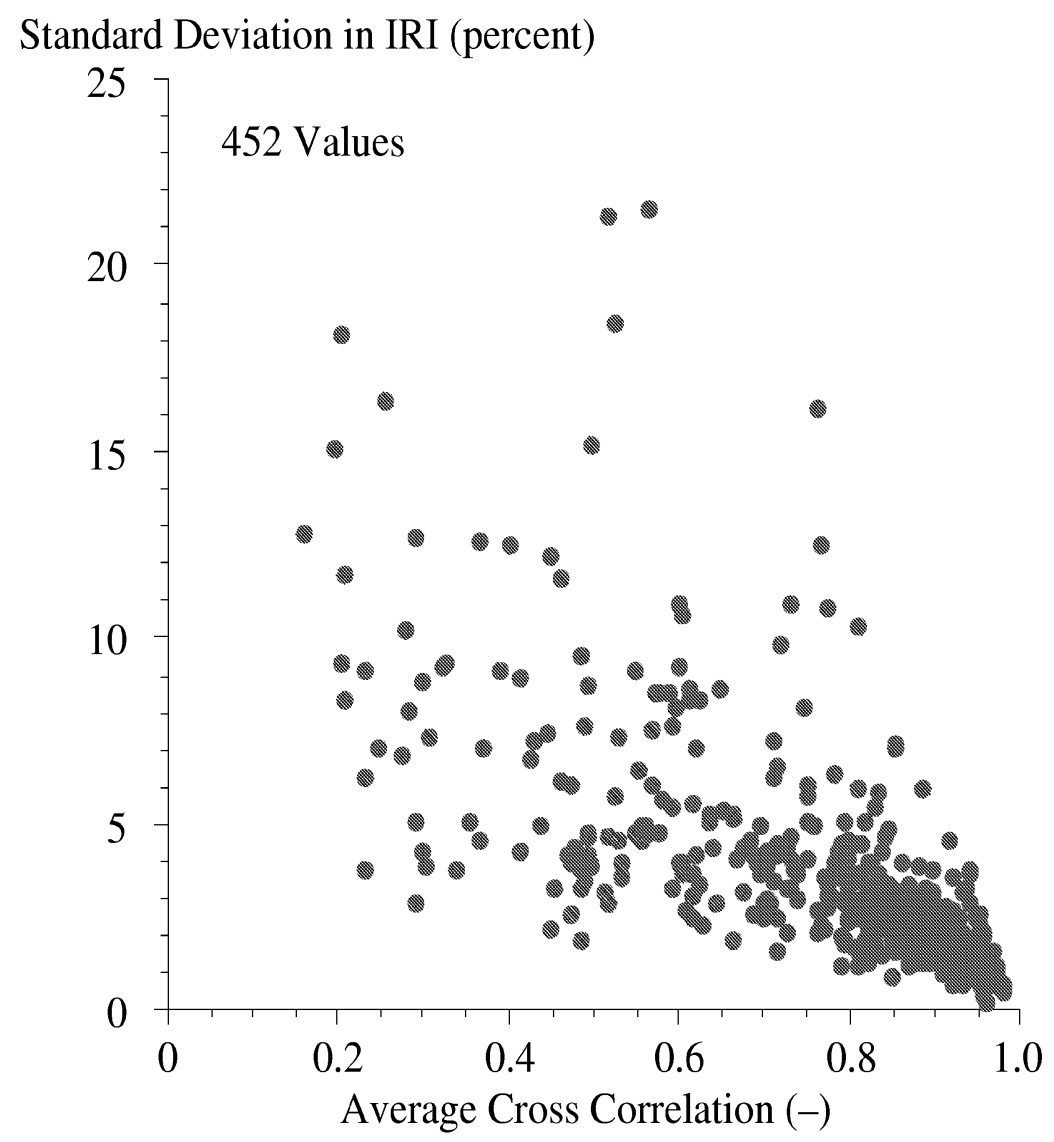

Figure C-1. Comparison of IRI scatter and cross correlation.

The values that occupy the lower left side of figure C-1 represent cases where the profiles did not agree very well, but consistent IRI values were observed. For example, device P76 measured the left wheeltrack of site 3 with an average cross correlation level of 0.51 , but the 10 measurements only had a standard deviation of 3.2 percent of their average. The output of the IRI filter for part of three of the measurements are shown in figure $\mathrm{C}-2$. Even though the overall IRI values are very close, the profiles agree very poorly. These profiles could certainly not be used to study the distribution of roughness within the site. Much of the apparent roughness in these profiles is noise. The agreement in IRI is simply due to the fact that the same amount of noise appears in each measurement. The low cross correlation value casts doubt on the accuracy of the IRI values, even though they are similar. The low correlation value may also indicate that this profiler can not be relied upon to produce repeatable IRI values on other sites of similar roughness content and surface texture.

In contrast, device P01 measured the left wheeltrack of site 3 with an average cross correlation level of 0.91 , and the 10 measurements had a standard deviation of 2.8 percent of their average. In this case the high level of repeatability in IRI measurement is a direct consequence of good repeatability in profile measurement. The output of the IRI filter for part of three of these measurements are shown in figure $\mathrm{C}-3$. These three traces are very similar, and show high levels of IRI content of approximately the same magnitude and in the same locations. The high average cross correlation value of these measurements 
indicates that this profiler can be expected to produce repeatable IRI values on other sites of similar roughness content and surface texture.

The 10 measurements by device P01 were an average of 2.7 percent higher than the Dipstick measurement of the same site, but the 10 measurements by device P76 were an average of 53.0 percent higher than the Dipstick. Thus, even though device P76 produced overall IRI values that were repeatable, they exhibited a large upward bias.In this case, poor repeatability of filtered profile served as a warning of poor accuracy in the summary index values.

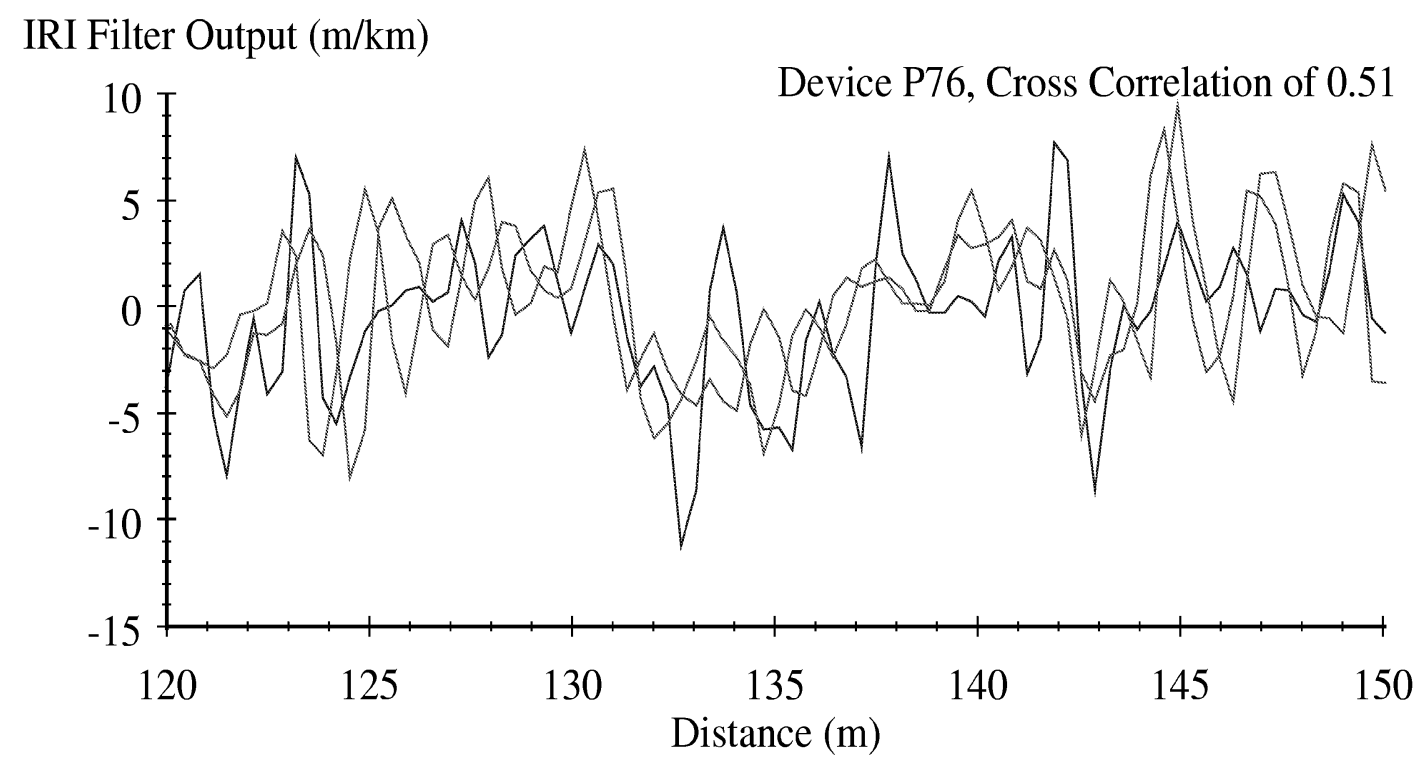

Figure C-2. Poorly-correlated IRI filter output.

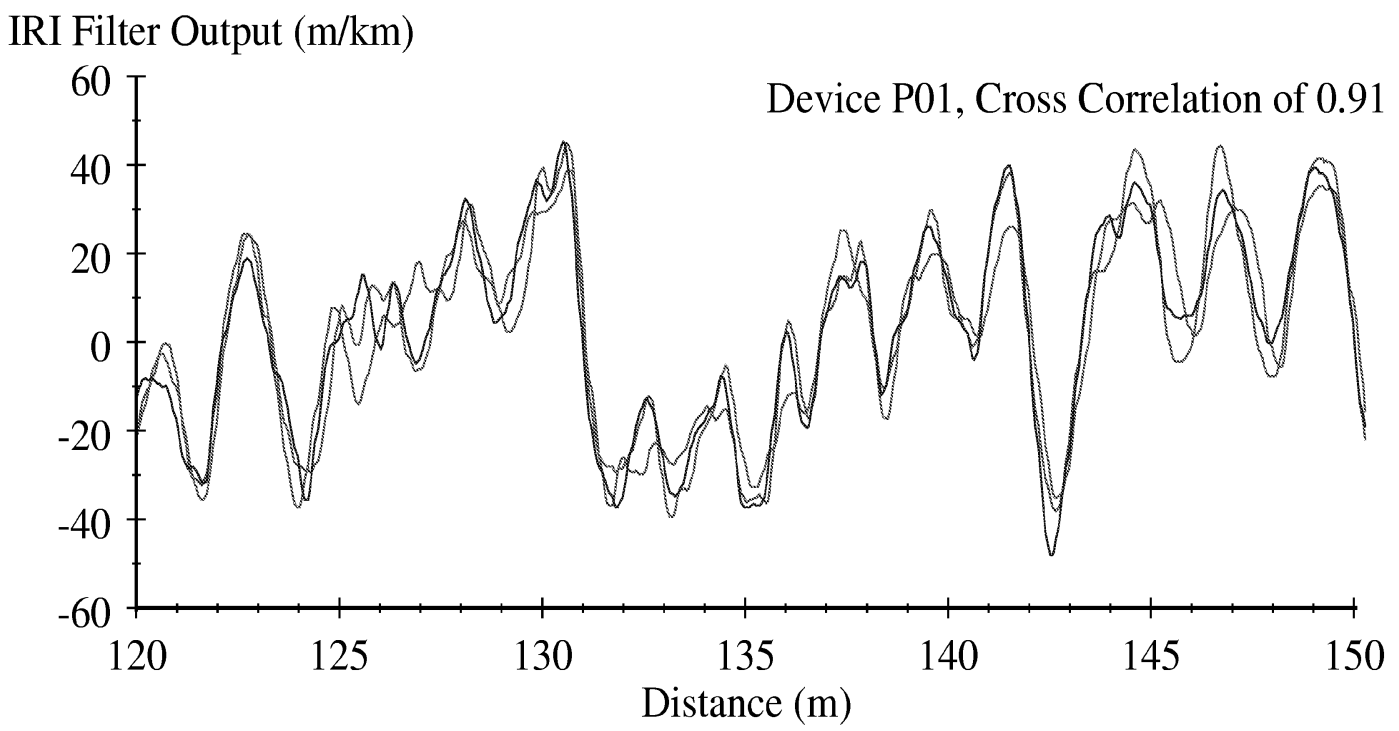

Figure C-3. Well-correlated IRI filter output. 


\section{AGREEMENT TO DIPSTICK}

In Appendix A, composite levels of agreement to the Dipstick in IRI measurement were calculated for each of 33 inertial profilers from the 1993 RPUG study. Each individual measured IRI value was compared to the corresponding Dipstick value. Most devices made 10 measurements on both wheeltracks of 8 sites. Thus, the 10 individual IRI values for a given wheeltrack were compared to the appropriate Dipstick value. This was repeated for each site (and wheeltrack), and all of the values (usually 160) of percent difference from the Dipstick were assembled into a distribution for that device. (See figure A-2.) The rootmean-square (RMS) "error" exhibited by each device is listed in tables C-3 and C-4. ${ }^{3}$ These values quantify a combination of bias and scatter in the IRI values. Bias was also calculated as an indicator of agreement to the Dipstick. This was the average percent difference between each IRI value and the appropriate Dipstick measurement. (Appendix A describes the compilation of these values in detail.)

The tables also list the average cross correlation values for each device when all of their measurements of a given wheeltrack were compared to the Dipstick profile. In this case, the IRI filter was used in the procedure. These values are the average of all cross correlation values for all repeat measurements. When 10 repeat measurements were made, the corresponding value in the table represents the average of 10 cross correlation values. Table C-3 lists the results for the left wheeltrack and table $\mathrm{C}-4$ lists the results for the right. (Not every device measured both wheeltracks, and some devices did not measure some of the sites at all.)

Overall, a vague relationship exists between the ratings of agreement and the cross correlation values. The lowest level of RMS error (5.1 percent) in table C-3 is exhibited by device N09, and is accompanied by cross correlation values for the 12 wheeltracks range from 0.79 to 0.95 . The highest level of RMS error ( 71.7 percent) is exhibited by device S06, and is accompanied by some of the lowest cross correlation values. Note that device N08 exhibits a low level of bias, but a high RMS error, and the cross correlation values are somewhat poor.

An important observation to be made about the cross correlation values is that they depend on the test site as well as the device. Several of the profilers have high cross correlation levels on some sites and low levels on others. This is because some sites are more problematic to some profilers than others. Since the listed standard deviation values often pertain to both wheeltracks of all sites, a direct comparison to cross correlation level is not possible.

To remedy this, an RMS error value and a bias level was calculated to correspond to each value for cross correlation in tables $\mathrm{C}-3$ and $\mathrm{C}-4$. Thus, new values were calculated that only include the error in measurement of IRI on one wheeltrack of one site by one device. Usually, this is the composite of 10 values, representing 10 repeat measurements.

33 The term "error" is in quotes because the Dipstick, although it was deemed a reference device at the time, is not guaranteed to provide the true IRI value. 
When this is done, all 452 cross correlation values in the tables can be paired to a level of RMS error and bias in IRI for the appropriate combination of device and wheeltrack.

Table C-3. Agreement to Dipstick in measurement of IRI, left.

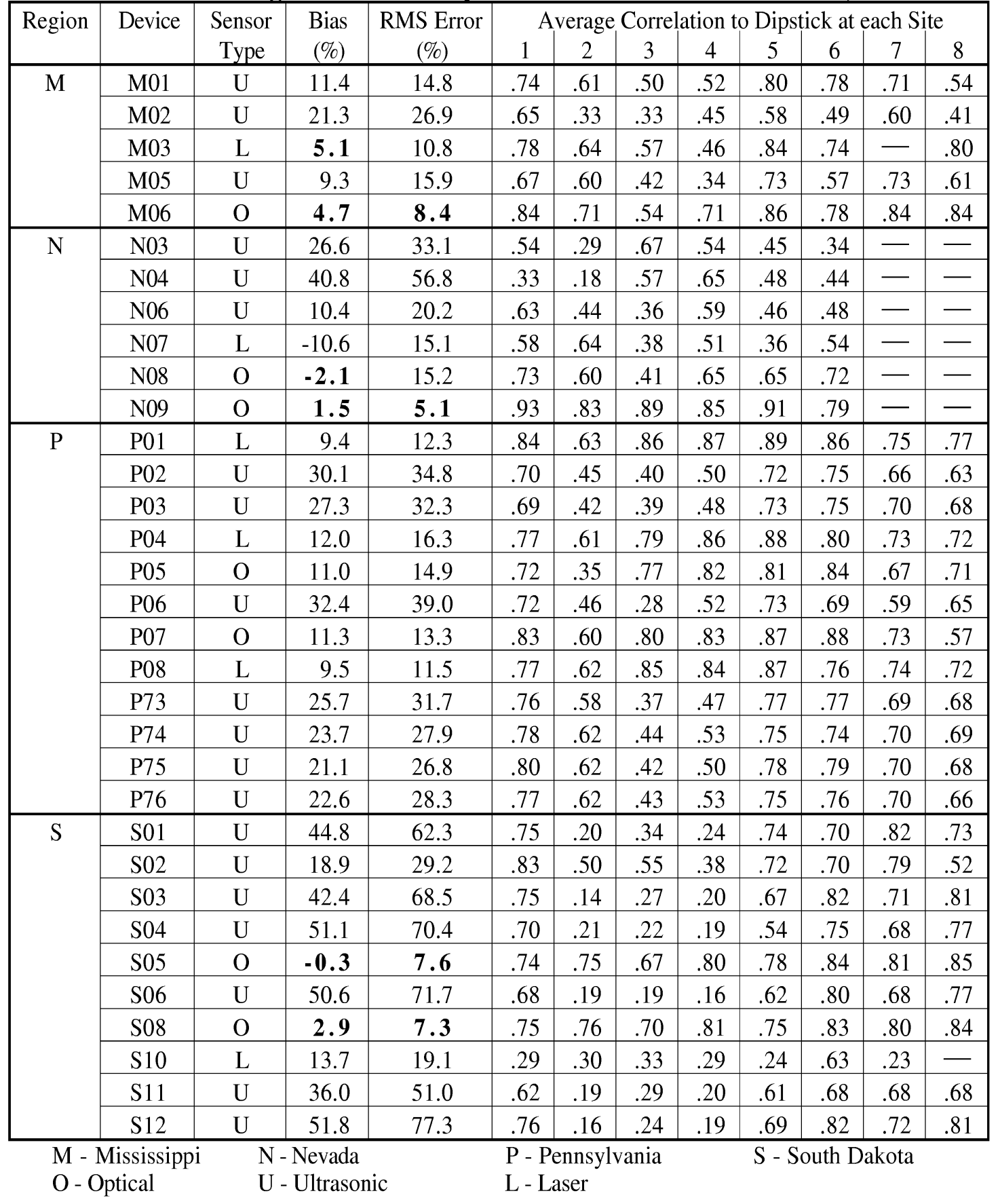


Table C-4. Agreement to Dipstick in measurement of IRI, right.

\begin{tabular}{|c|c|c|c|c|c|c|c|c|c|c|c|c|}
\hline \multirow[t]{2}{*}{ Region } & \multirow[t]{2}{*}{ Device } & \multirow{2}{*}{$\begin{array}{c}\text { Sensor } \\
\text { Type }\end{array}$} & \multirow{2}{*}{$\begin{array}{l}\text { Bias } \\
(\%)\end{array}$} & \multirow{2}{*}{$\begin{array}{c}\text { RMS Error } \\
(\%)\end{array}$} & \multicolumn{8}{|c|}{ Average Correlation to Dipstick at each Site } \\
\hline & & & & & 1 & 2 & 3 & 4 & 5 & 6 & 7 & 8 \\
\hline \multirow[t]{4}{*}{$\mathrm{M}$} & M02 & $\mathrm{U}$ & 21.3 & 26.9 & .58 & .47 & .30 & .46 & .52 & .60 & .61 & .47 \\
\hline & M03 & $\mathrm{L}$ & 5.1 & 10.8 & .83 & .86 & .62 & .67 & .76 & .84 & - & .81 \\
\hline & M05 & $\mathrm{U}$ & 9.3 & 15.9 & .74 & .69 & .34 & .51 & .66 & .69 & .69 & .63 \\
\hline & M06 & $\mathrm{O}$ & 4.7 & 8.4 & .76 & .80 & .47 & .77 & .80 & .86 & .89 & .80 \\
\hline \multirow[t]{6}{*}{$\mathrm{N}$} & N03 & $\mathrm{U}$ & 26.6 & 33.1 & .68 & .34 & .43 & .58 & .48 & .42 & - & - \\
\hline & N04 & $\mathrm{U}$ & 40.8 & 56.8 & .41 & .21 & .24 & .64 & .50 & .54 & - & - \\
\hline & N06 & $\mathrm{U}$ & 10.4 & 20.2 & .76 & .38 & .33 & .56 & .54 & .55 & - & - \\
\hline & N07 & $\mathrm{L}$ & -10.6 & 15.1 & .76 & .59 & .51 & .58 & .47 & .64 & - & - \\
\hline & N08 & $\mathrm{O}$ & -2.1 & 15.2 & .87 & .75 & .68 & .79 & .60 & .70 & - & - \\
\hline & N09 & $\mathrm{O}$ & 1.5 & 5.1 & .93 & .95 & .92 & .87 & .93 & .90 & - & - \\
\hline \multirow[t]{12}{*}{$\mathrm{P}$} & P01 & $\mathrm{L}$ & 9.4 & 12.3 & .85 & .74 & .69 & .89 & .90 & .76 & .78 & .70 \\
\hline & P02 & $\mathrm{U}$ & 30.1 & 34.8 & .71 & .43 & .33 & .59 & .72 & .68 & .72 & .57 \\
\hline & $\mathrm{P} 03$ & $\mathrm{U}$ & 27.3 & 32.3 & .71 & .36 & .46 & .52 & .68 & .67 & .74 & .61 \\
\hline & P04 & $\mathrm{L}$ & 12.0 & 16.3 & .78 & .63 & .78 & .78 & .86 & .64 & .76 & .66 \\
\hline & P05 & $\mathrm{O}$ & 11.0 & 14.9 & .78 & .71 & .77 & .86 & .81 & .72 & .83 & .75 \\
\hline & P06 & $\mathrm{U}$ & 32.4 & 39.0 & .73 & .43 & .38 & .66 & .70 & .67 & .73 & .64 \\
\hline & P07 & $\mathrm{O}$ & 11.3 & 13.3 & .79 & .71 & .68 & .77 & .82 & .74 & .80 & .82 \\
\hline & P08 & $\mathrm{L}$ & 9.5 & 11.5 & .83 & .74 & .73 & .92 & .87 & .71 & .81 & .76 \\
\hline & P73 & $\mathrm{U}$ & 25.7 & 31.7 & .78 & .51 & .37 & .57 & .74 & .68 & .74 & .64 \\
\hline & P74 & $\mathrm{U}$ & 23.7 & 27.9 & .79 & .61 & .43 & .54 & .69 & .68 & .72 & .62 \\
\hline & P75 & $\mathrm{U}$ & 21.1 & 26.8 & .83 & .61 & .42 & .68 & .76 & .70 & .74 & .62 \\
\hline & P76 & $\mathrm{U}$ & 22.6 & 28.3 & .78 & .71 & .37 & .72 & .74 & .70 & .75 & .63 \\
\hline \multirow[t]{5}{*}{$S$} & S01 & $\mathrm{U}$ & 44.8 & 62.3 & .72 & .35 & .36 & .30 & .75 & .69 & .82 & .74 \\
\hline & S05 & $\mathrm{O}$ & -0.3 & 7.6 & .82 & .86 & .81 & .79 & .87 & .88 & .95 & .88 \\
\hline & S08 & $\mathrm{O}$ & 2.9 & 7.3 & .78 & .85 & .77 & .80 & .85 & .88 & .91 & .90 \\
\hline & $\mathrm{S} 10$ & $\mathrm{~L}$ & 13.7 & 19.1 & .44 & .51 & .45 & .38 & .21 & .70 & .33 & - \\
\hline & S11 & $\mathrm{U}$ & 36.0 & 51.0 & .60 & .34 & .31 & .27 & .68 & .71 & .76 & .77 \\
\hline \multicolumn{3}{|c|}{ M - Mississippi } & $\begin{array}{l}\text { Nevada } \\
\text { Jltrason }\end{array}$ & & $\begin{array}{l}P-1 \\
L-I\end{array}$ & & & & & th D & & \\
\hline
\end{tabular}

Figure C-4 compares the RMS error levels to the level of cross correlation. When the cross correlation level is near 1, the RMS error is near zero. This is because a cross correlation level of 1 indicates total agreement in the components of profile that affect the IRI. As the cross correlation level decreases, the largest observed RMS error in IRI measurement increases. This is because the profiles themselves do not agree as well. In addition, when cross correlation decreases too far the potential for agreement in IRI caused by compensating error increases, so the range of RMS error values spans a range from zero to some large value. In this case, a cross correlation level of 0.90 is required to guarantee an RMS error level below 5 percent.

\section{Agreement Between ANy PaIR OF Measurements}

The discussions of repeatability and agreement to the Dipstick above show that there is a relationship between error in overall IRI measurement and the cross correlation level between profiles. Unfortunately, those data are not comprehensive enough to permit the association of a given level of cross correlation with a desired expected error level in IRI. 


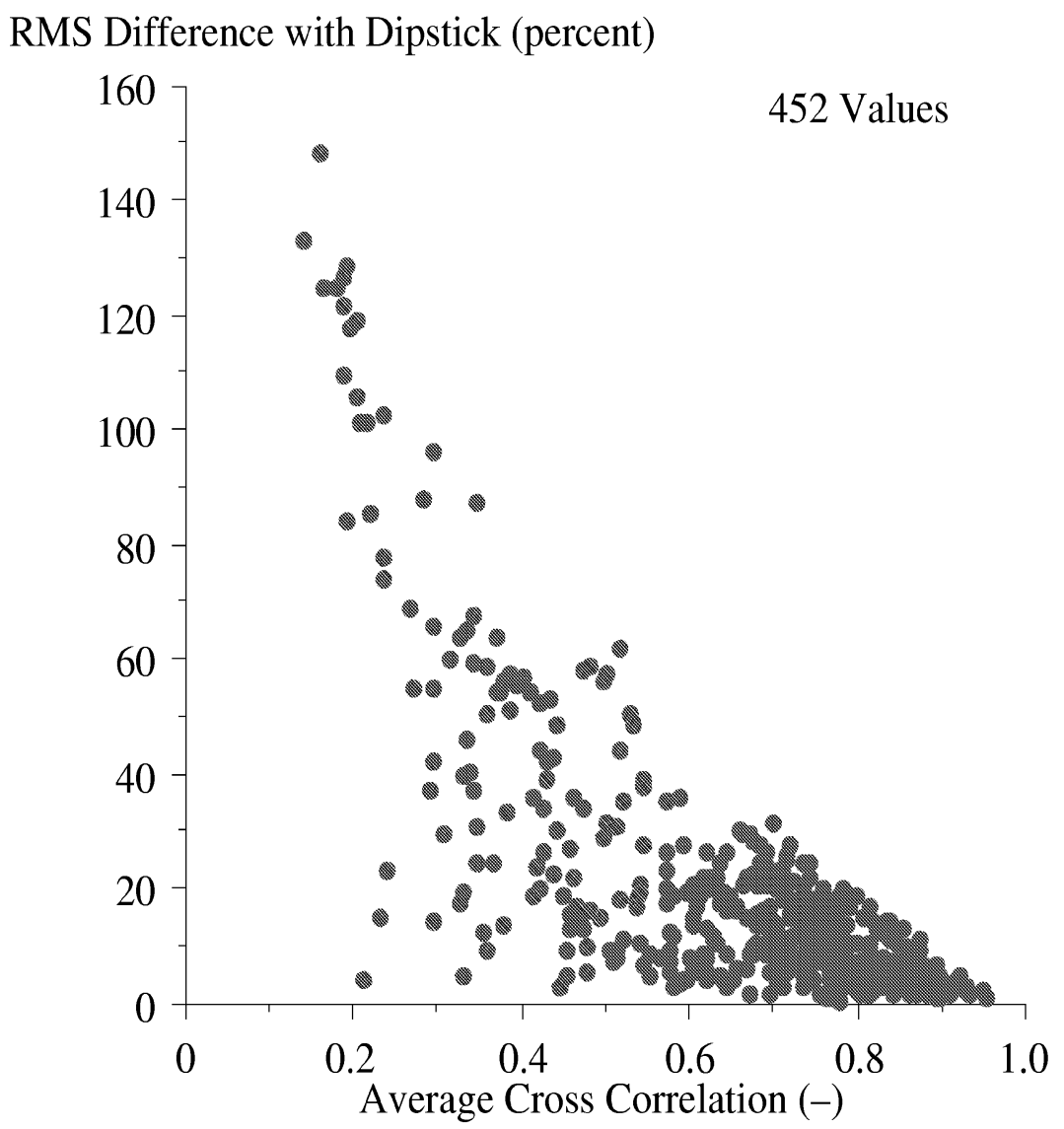

Figure C-4. Comparison of RMS error in IRI and cross correlation.

To help establish this relationship, all possible comparisons of individual measurements from the 1993 RPUG study were examined as individual samples of the relationship. To do this, any pair of profiles that covered the same wheeltrack were cross correlated using IRI filter output. The level of cross correlation was then "paired" with the percent difference in IRI.

The calculation of IRI error and cross correlation level for all possible combinations resulted in 374,758 pairs of values. These pairs include comparisons of Dipstick with inertial profilers, inertial profilers with their own repeat measurements, and comparisons across different inertial profilers. For example, the left wheeltrack of site 4 in Pennsylvania was measured by 12 profilers 10 times each, and once by the Dipstick. (See table B-1.) These 121 measurements permit 14,520 individual comparisons. Note that each pair was compared twice, so that one of the profiles could take the role of reference measurement in each comparison. This was needed because the process does not have reciprocity.

Once the level of IRI error and cross correlation were computed for all pairs, they were assembled into bins by their cross correlation level. Each bin covered a range of 0.01 (out of 1) along the scale. For example, the bin that ranged from 0.93 to 0.94 included 3,394 pairs. The distribution of IRI error level within this bin is shown in figure $\mathrm{C}-5$.

The content of this distribution can be summarized in five ways:

1. Average: The average value of IRI error. This should be small, but it will always be greater than zero. (Consider a pair of IRI measurements of $1.00 \mathrm{~m} / \mathrm{km}$ and 1.05 
$\mathrm{m} / \mathrm{km}$. They will yield two values of error: 5 percent and -4.76 percent. This nonlinearity will cause the average error level to have a non-zero value.)

2. RMS: The root mean square error level includes the influence of upward and downward errors. When it is considered in conjunction with the average error level, it would be enough to define the distribution if the Gaussian assumption was reasonable. In figure $\mathrm{C}-5$, this may not be the case.

3. 95th Percentile: The 95th percentile error level includes the influence of upward and downward errors also. A desired expected 95th percentile error level in IRI should be used to set a minimum cross correlation level between profiles.

4. Maximum Error: This is the maximum level of IRI upward bias error observed within the range of cross correlation level under examination. It is simply included to place a bound on the possible error level, and warn users of these results of the inevitable anecdote that a measurement with a high level of cross correlation still showed some disagreement in IRI.

5. Minimum Error: This is the maximum level of IRI downward bias error observed within the range of cross correlation level of under examination.

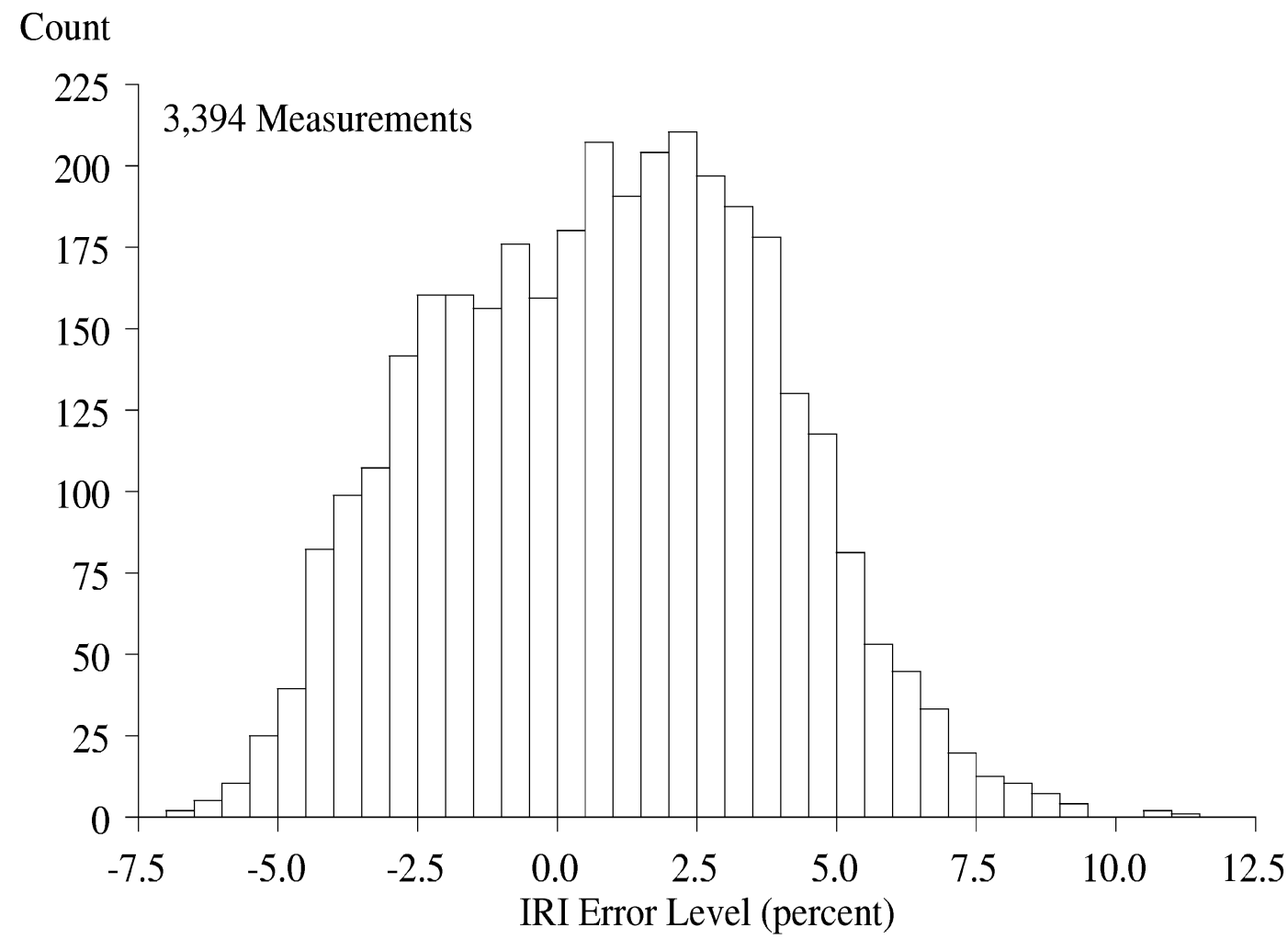

Figure C-5. Distribution of IRI disagreement, cross correlation 0.93-0.94.

These five quantities are displayed in figure C-6 for all 100 possible bins by upper cross correlation limit. The values displayed in figure $\mathrm{C}-6$ are also listed in table $\mathrm{C}-5$, below, so that individual values of interest are easier to read. The table also lists the number of pairs within each bin. The error levels grow quite high when the cross correlation level decreases below about 0.80 . The figure is therefore repeated for values of cross correlation above 0.85 in figure $\mathrm{C}-7$. 


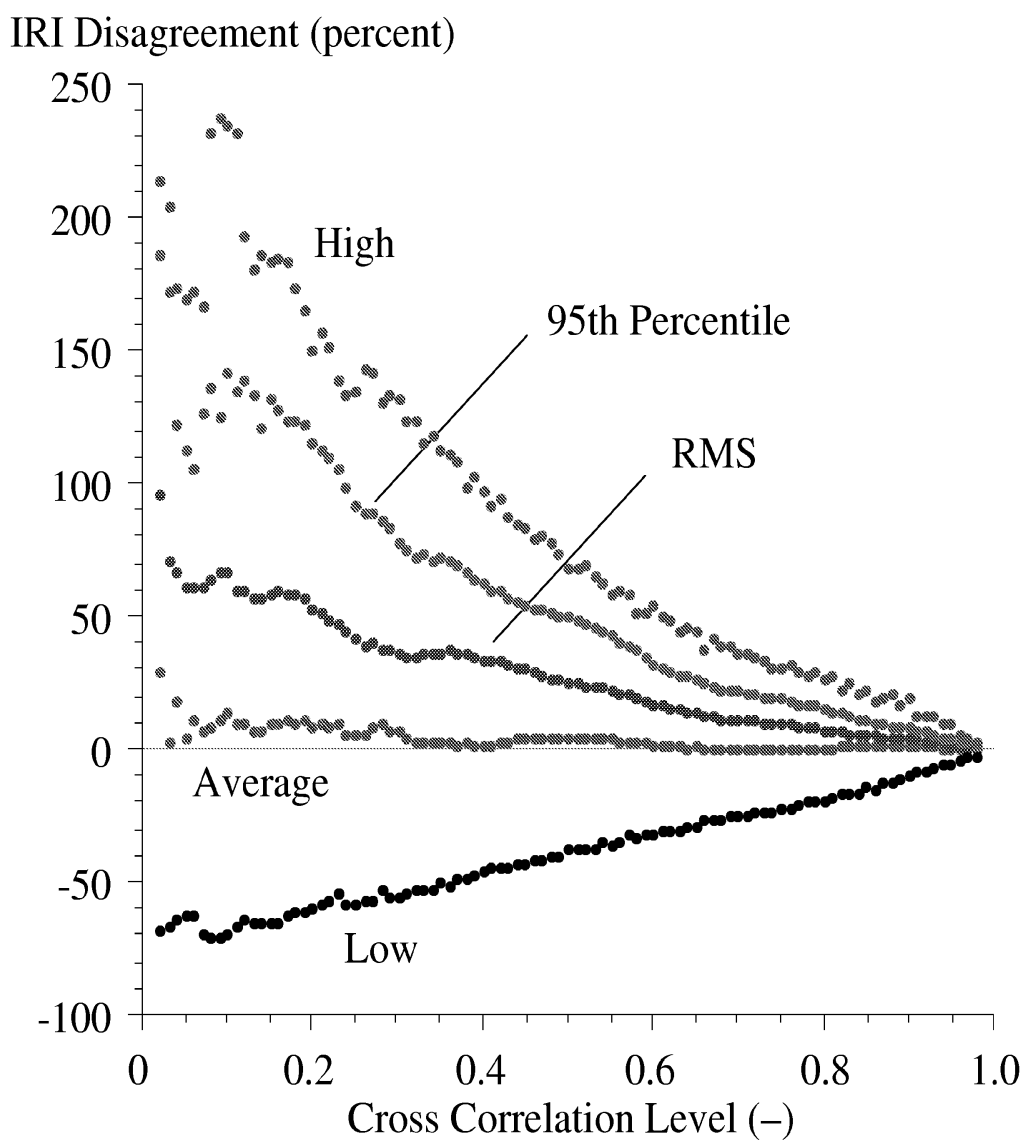

Figure C-6. IRI disagreement at various cross correlation levels.

Road profiling technology has improved significantly since the 1993 RPUG experiment. Unfortunately, the 1993 RPUG data were the only source of repeat measurements by several profilers on the same site at the time this research was done. It is recommended that the same study is performed on data from modern profilers. The use of ultrasonic sensors has been discredited since 1993, in part due to the RPUG experiment. Thus, the analyses described above were repeated without any measurements from profilers with ultrasonic sensors. In this case, only 129,812 pairs were available. Figure C -8 and table $\mathrm{C}-6$ provide the results. In the range of desirable performance, the results did not change significantly.

It is recommended that, until a more relevant data set can be obtained, cross correlation limits are set of various "classes" of profiler based on these values. For example, at a cross correlation level of 0.98 , you may expect 95 percent of your IRI measurements to agree within 2 percent. This could be proposed as a threshold limit for a "research class" profiler. At a cross correlation level of 0.94 , you may expect 95 percent of your IRI measurements to agree within 5 percent. This could be proposed as a threshold limit for a "project class" profiler. The same limit could be proposed for construction quality control, as long as the test sites were carefully chosen to duplicate the smoothness and texture expected in the field. Lastly, at a cross correlation level of 0.88 , you may expect 95 percent of your IRI measurements to agree within 10 percent of each other. This could be proposed as a threshold limit for a "network class" profiler. 


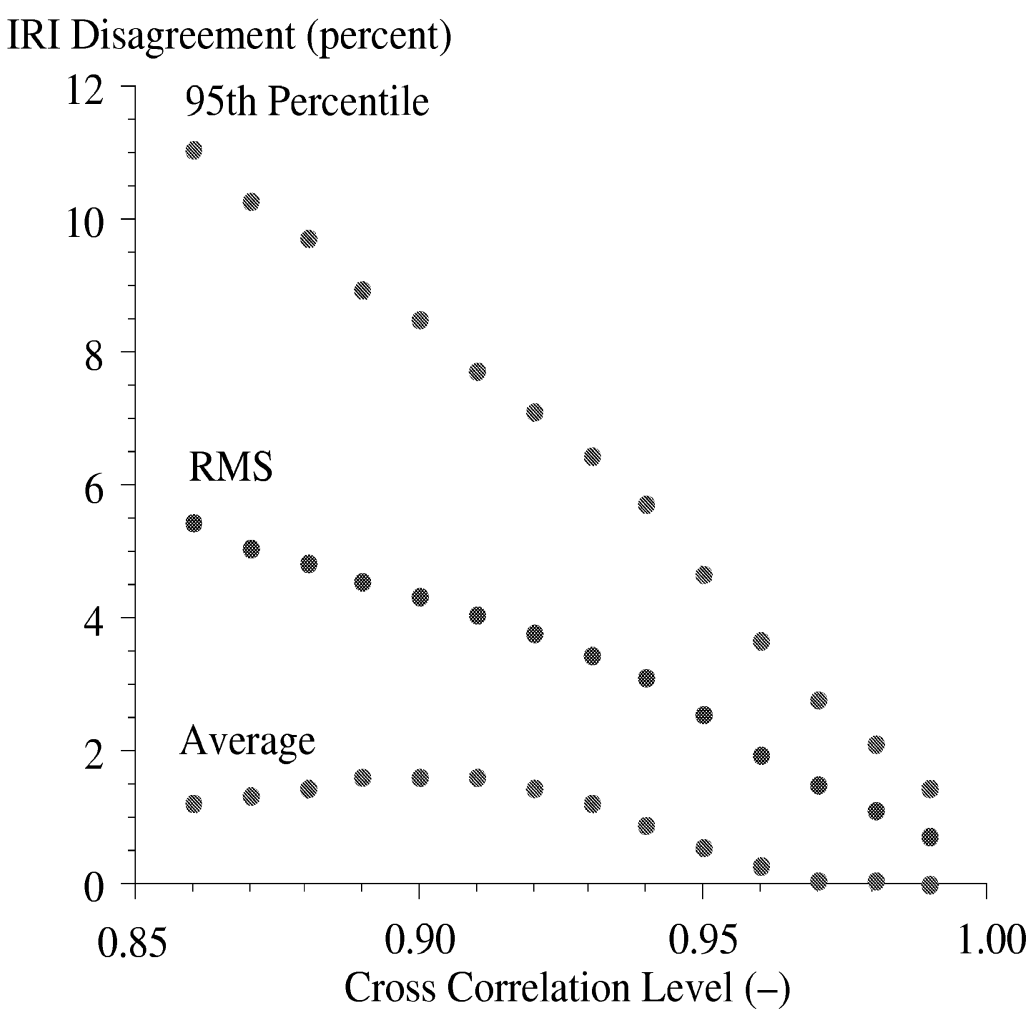

Figure C-7. IRI disagreement at high cross correlation levels.

IRI Disagreement (percent)

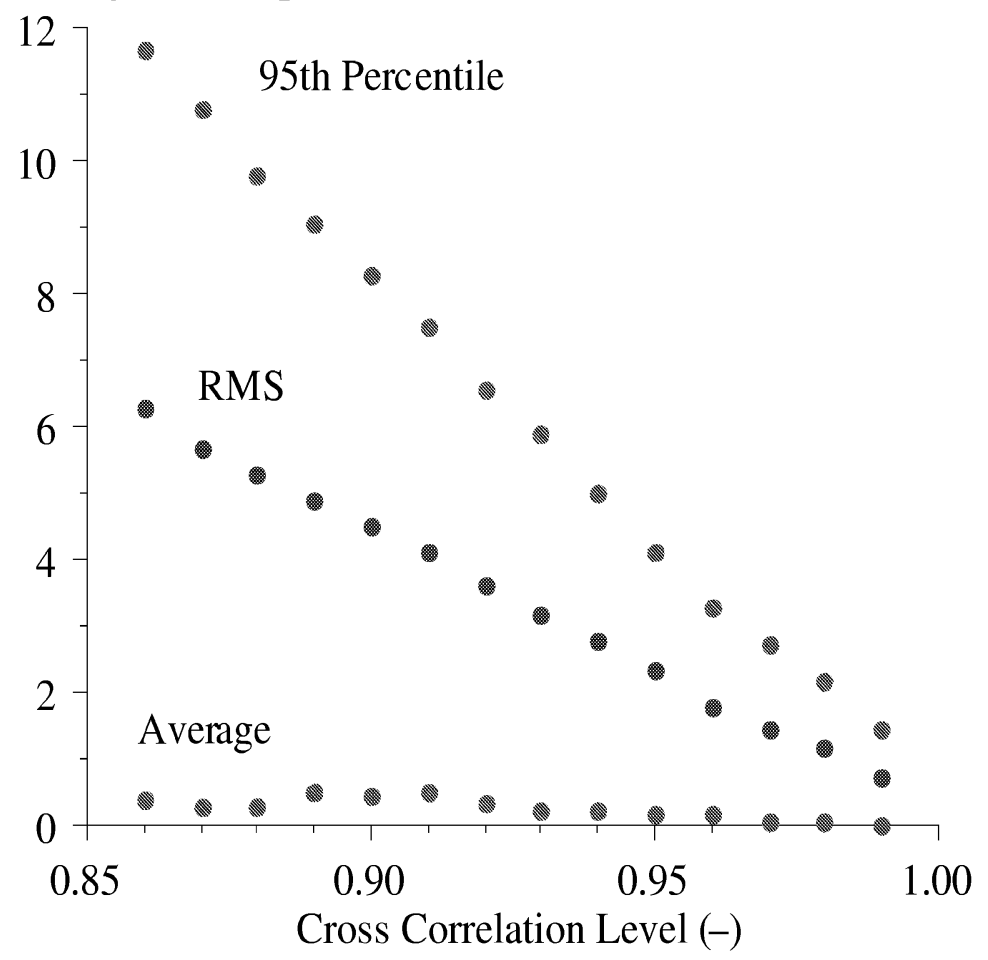

Figure C-8. IRI disagreement associated with cross correlation level, ultrasonic devices excluded. 
Table C-5. Expected IRI error associated with cross correlation level.

\begin{tabular}{|c|c|c|c|c|c|c|c|}
\hline \multicolumn{2}{|c|}{$\begin{array}{c}\text { Cross Correlation } \\
\text { Range }\end{array}$} & \multirow{2}{*}{$\begin{array}{l}\text { Number of } \\
\text { Comparisons }\end{array}$} & \multicolumn{5}{|c|}{ Error Level in IRI Measurement (Percent) } \\
\hline From & To & & Average & RMS & $\begin{array}{c}\text { 95th } \\
\text { Percentile }\end{array}$ & Low & High \\
\hline .98 & .99 & 877 & 0.01 & 0.74 & 1.47 & -2.22 & 3.39 \\
\hline .97 & .98 & 1543 & 0.03 & 1.14 & 2.14 & -3.24 & 3.65 \\
\hline .96 & .97 & 1956 & 0.07 & 1.48 & 2.76 & -4.10 & 5.20 \\
\hline .95 & .96 & 2393 & 0.26 & 1.96 & 3.65 & -5.57 & 9.47 \\
\hline .94 & .95 & 2902 & 0.54 & 2.55 & 4.69 & -5.89 & 10.16 \\
\hline .93 & .94 & 3394 & 0.89 & 3.12 & 5.74 & -6.82 & 12.43 \\
\hline .92 & .93 & 3704 & 1.20 & 3.46 & 6.45 & -7.69 & 12.49 \\
\hline .91 & .92 & 4677 & 1.45 & 3.79 & 7.09 & -8.43 & 13.03 \\
\hline .90 & .91 & 5753 & 1.61 & 4.07 & 7.73 & -10.28 & 18.76 \\
\hline .89 & .90 & 6707 & 1.61 & 4.32 & 8.49 & -10.73 & 16.95 \\
\hline .88 & .89 & 7522 & 1.60 & 4.57 & 8.96 & -12.14 & 20.56 \\
\hline .87 & .88 & 8369 & 1.47 & 4.83 & 9.71 & -12.21 & 19.38 \\
\hline .86 & .87 & 9051 & 1.33 & 5.06 & 10.29 & -14.70 & 18.69 \\
\hline .85 & .86 & 9380 & 1.20 & 5.42 & 11.06 & -14.23 & 21.81 \\
\hline .84 & .85 & 9768 & 0.95 & 5.68 & 11.52 & -16.01 & 20.30 \\
\hline .83 & .84 & 9941 & 0.91 & 6.12 & 12.49 & -16.62 & 24.70 \\
\hline .82 & .83 & 9718 & 0.83 & 6.38 & 13.26 & -16.57 & 22.12 \\
\hline .81 & .82 & 9695 & 0.64 & 6.91 & 14.19 & -17.93 & 27.35 \\
\hline .80 & .81 & 9450 & 0.69 & 7.48 & 15.61 & -19.12 & 25.94 \\
\hline .79 & .80 & 9179 & 0.68 & 7.90 & 16.18 & -18.99 & 29.13 \\
\hline .78 & .79 & 9046 & 0.52 & 8.29 & 16.73 & -19.49 & 27.88 \\
\hline .77 & .78 & 8572 & 0.52 & 8.73 & 17.27 & -21.43 & 29.13 \\
\hline .76 & .77 & 8146 & 0.56 & 9.03 & 17.88 & -22.19 & 31.30 \\
\hline .75 & .76 & 8147 & 0.33 & 9.58 & 18.85 & -22.56 & 30.23 \\
\hline .74 & .75 & 7609 & 0.49 & 10.11 & 19.58 & -23.19 & 31.13 \\
\hline .73 & .74 & 7352 & 0.17 & 10.19 & 19.88 & -23.03 & 33.78 \\
\hline .72 & .73 & 6986 & 0.17 & 10.61 & 20.77 & -23.49 & 34.64 \\
\hline .71 & .72 & 6675 & 0.25 & 10.93 & 21.17 & -24.73 & 35.47 \\
\hline .70 & .71 & 6350 & 0.06 & 11.12 & 21.79 & -25.06 & 36.00 \\
\hline .69 & .70 & 6092 & 0.31 & 11.45 & 22.45 & -25.43 & 38.64 \\
\hline .68 & .69 & 5869 & 0.44 & 11.73 & 22.72 & -25.81 & 38.44 \\
\hline .67 & .68 & 5588 & 0.45 & 12.47 & 23.99 & -26.18 & 41.36 \\
\hline .66 & .67 & 5207 & 0.53 & 12.81 & 24.49 & -26.19 & 37.88 \\
\hline .65 & .66 & 4989 & 1.07 & 13.34 & 26.15 & -28.49 & 44.84 \\
\hline .64 & .65 & 4835 & 0.67 & 14.15 & 27.42 & -29.33 & 46.13 \\
\hline .63 & .64 & 4493 & 0.94 & 14.62 & 27.87 & -30.23 & 45.14 \\
\hline .62 & .63 & 4313 & 0.91 & 15.35 & 29.11 & -30.96 & 48.25 \\
\hline .61 & .62 & 4273 & 1.35 & 16.30 & 30.81 & -30.62 & 49.46 \\
\hline .60 & .61 & 4245 & 1.41 & 17.19 & 32.43 & -32.26 & 53.58 \\
\hline
\end{tabular}

129,812 comparison are excluded, because their cross correlation level is outside the limits of the table. 
Table C-6. Expected IRI error associated with cross correlation level, ultrasonic devices excluded.

\begin{tabular}{|c|c|c|c|c|c|c|c|}
\hline \multirow{2}{*}{\multicolumn{2}{|c|}{$\begin{array}{c}\text { Cross Correlation } \\
\text { Range }\end{array}$}} & \multirow{3}{*}{$\begin{array}{l}\text { Number of } \\
\text { Comparisons }\end{array}$} & \multicolumn{5}{|c|}{ Error Level in IRI Measurement (Percent) } \\
\hline & & & & & & & \\
\hline From & To & & Average & RMS & $\begin{array}{c}\text { 95th } \\
\text { Percentile }\end{array}$ & Low & High \\
\hline .98 & .99 & 871 & 0.01 & 0.74 & 1.47 & -2.22 & 3.39 \\
\hline 97 & .98 & 1534 & 0.03 & 1.14 & 2.14 & -3.24 & 3.65 \\
\hline 96 & .97 & 1906 & 0.05 & 1.47 & 2.74 & -4.10 & 5.20 \\
\hline 95 & .96 & 2184 & 0.14 & 1.79 & 3.28 & -4.59 & 6.46 \\
\hline .94 & .95 & 2320 & 0.18 & 2.31 & 4.10 & -5.72 & 8.33 \\
\hline 93 & .94 & 2302 & 0.20 & 2.78 & 4.98 & -6.82 & 9.24 \\
\hline .92 & .93 & 2102 & 0.24 & 3.15 & 5.87 & -7.69 & 11.46 \\
\hline .91 & .92 & 2296 & 0.32 & 3.63 & 6.58 & -8.43 & 12.02 \\
\hline .90 & .91 & 2314 & 0.50 & 4.12 & 7.48 & -10.28 & 12.42 \\
\hline .89 & .90 & 2415 & 0.46 & 4.48 & 8.26 & -10.73 & 12.27 \\
\hline .88 & .89 & 2342 & 0.52 & 4.90 & 9.06 & -11.04 & 14.79 \\
\hline .87 & .88 & 2384 & 0.30 & 5.30 & 9.79 & -12.15 & 16.41 \\
\hline .86 & .87 & 2273 & 0.26 & 5.67 & 10.76 & -12.49 & 16.25 \\
\hline .85 & .86 & 2257 & 0.37 & 6.28 & 11.68 & -12.76 & 21.81 \\
\hline .84 & .85 & 2287 & 0.22 & 6.60 & 12.31 & -13.98 & 19.61 \\
\hline .83 & .84 & 2121 & 0.34 & 6.90 & 13.06 & -15.51 & 19.57 \\
\hline .82 & .83 & 1915 & 0.18 & 6.82 & 12.76 & -15.83 & 21.71 \\
\hline .81 & .82 & 1888 & -0.10 & 6.95 & 13.14 & -16.61 & 19.92 \\
\hline .80 & .81 & 1684 & 0.09 & 7.15 & 13.62 & -16.84 & 21.62 \\
\hline .79 & .80 & 1701 & 0.16 & 7.47 & 14.46 & -17.41 & 22.14 \\
\hline .78 & .79 & 1626 & 0.38 & 7.65 & 14.72 & -18.38 & 22.52 \\
\hline .77 & .78 & 1617 & 0.38 & 8.05 & 15.33 & -18.41 & 22.57 \\
\hline .76 & .77 & 1395 & 0.53 & 8.07 & 15.29 & -18.93 & 23.85 \\
\hline .75 & .76 & 1366 & 0.12 & 8.69 & 16.42 & -17.96 & 22.21 \\
\hline .74 & .75 & 1137 & 0.29 & 9.23 & 17.41 & -19.80 & 27.23 \\
\hline .73 & .74 & 1090 & -0.18 & 9.55 & 18.01 & -21.44 & 28.10 \\
\hline .72 & .73 & 903 & 0.25 & 9.76 & 17.97 & -21.94 & 28.88 \\
\hline .71 & .72 & 798 & 0.38 & 10.74 & 19.53 & -23.42 & 30.59 \\
\hline .70 & .71 & 740 & -0.17 & 10.08 & 18.12 & -19.96 & 28.76 \\
\hline .69 & .70 & 745 & 0.82 & 11.15 & 20.00 & -22.41 & 30.38 \\
\hline .68 & .69 & 681 & 0.65 & 11.65 & 20.81 & -22.98 & 29.69 \\
\hline .67 & .68 & 658 & 0.17 & 12.16 & 21.40 & -23.30 & 30.12 \\
\hline .66 & .67 & 597 & 0.52 & 12.39 & 23.13 & -23.64 & 34.68 \\
\hline .65 & .66 & 519 & 0.65 & 12.43 & 22.25 & -23.54 & 32.09 \\
\hline .64 & .65 & 616 & 0.87 & 12.83 & 24.30 & -24.97 & 33.51 \\
\hline .63 & .64 & 600 & 0.22 & 12.63 & 23.52 & -30.23 & 43.33 \\
\hline .62 & .63 & 592 & 1.60 & 12.02 & 23.26 & -27.54 & 38.00 \\
\hline .61 & .62 & 575 & 0.30 & 11.66 & 22.87 & -24.96 & 33.47 \\
\hline .60 & .61 & 516 & 0.31 & 12.63 & 26.40 & -30.91 & 44.75 \\
\hline
\end{tabular}

8,650 comparison are excluded, because their cross correlation level is outside the limits of the table. 